\title{
FUNCTIONAL MODIFICATION OF POLYMERIC NANOGELS
}

Disraëli Noami Marie-Theresse Kusmus 
This dissertation has been approved by:

Supervisor

prof. dr. ir. J.J.L.M. Cornelissen

Co-supervisor

dr. J.M.J. Paulusse

Cover design: Luca Ricciardi

Printed by: Gildeprint

Lay-out: Disraëli Kusmus

ISBN: 978-90-365-5283-7

DOI: $10.3990 / 1.9789036552837$

(C) 2022 Disraëli Noami Marie-Theresse Kusmus, The Netherlands. All rights reserved. No parts of this thesis may be reproduced, stored in a retrieval system or transmitted in any form or by any means without permission of the author. Alle rechten voorbehouden. Niets uit deze uitgave mag worden vermenigvuldigd, in enige vorm of op enige wijze, zonder voorafgaande schriftelijke toestemming van de auteur. 


\title{
FUNCTIONAL MODIFICATION OF POLYMERIC NANOGELS
}

\section{DISSERTATION}

\author{
to obtain \\ the degree of doctor at the Universiteit Twente, \\ on the authority of the rector magnificus, \\ prof. dr. ir. A. Veldkamp, \\ on account of the decision of the Doctorate Board \\ to be publicly defended \\ on Friday 18 February 2022 at 14.45 hours
}

by

Disraëli Noami Marie-Theresse Kusmus

born on the 3rd of April, 1989

in Willemstad, Curaçao 


\section{Graduation Committee:}

Chair / secretary:

Supervisor:

Co-supervisor:

Committee Members: prof. dr. J.L. Herek

prof. dr. ir. J.J.L.M. Cornelissen

dr. J.M.J. Paulusse

prof. dr. F.R. Wurm

dr. J.I. Paez

prof. dr. M. Kamperman

prof. dr. A. Herrmann 
"When you saw only one set of footprints, it was then that I carried you" 



\section{Table of contents}

Chapter 1

page 1

Controlled radical crosslinking polymerization of multivinyl monomers to form size-controlled nanogels

Chapter 2

page 15

Surface functionalizations and cellular uptake of nanogels in biomedical applications

Chapter 3

page 99

Uniquely sized nanogels via crosslinking polymerization

Chapter 4

page 133

Post-polymerization functionalization of the epoxy moiety to sulfonium nanogels

Chapter 5

page 167

Sulfonium-functionalized nanogels for gene delivery

Chapter 6

page 187

Transfection efficiency of sulfonium nanogels

Chapter 7

page 229

Perspectives of sulfonium nanogels in gene therapy

Summary

page 241

Samenvatting

page 243

Acknowledgements

page 245

About the author

page 249 
ii | P a g e 
Chapter 1

Controlled radical crosslinking polymerization of multivinyl monomers to form size-controlled nanogels

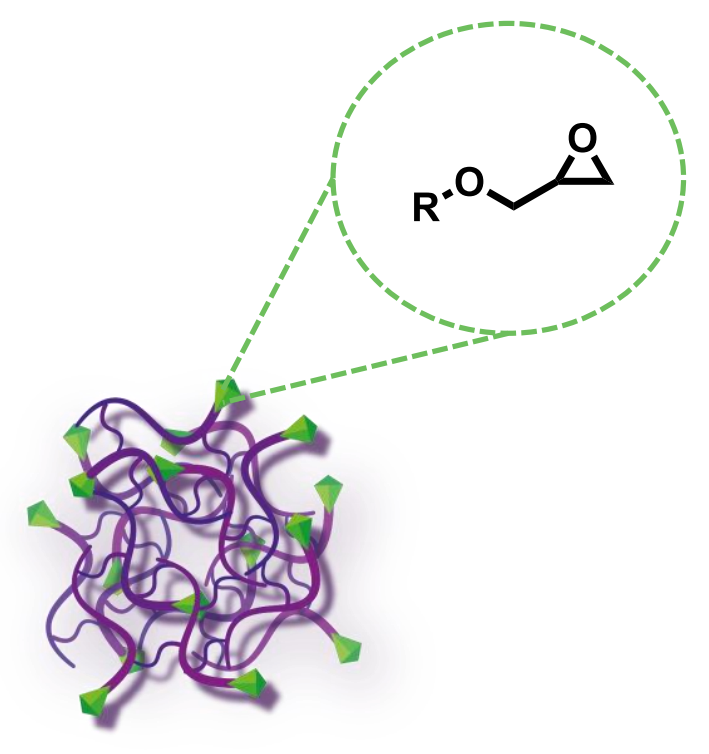




\subsection{General introduction}

Synthetic polymer chemistry has benefitted greatly from the emergence of controlled/living radical polymerization (CRP) such as atom transfer radical polymerization (ATRP), nitroxide-mediated radical polymerization (NMP) and reversible addition fragmentation chain transfer (RAFT). ${ }^{1-5}$ The use of multivinyl monomers (MVM) during CRP has realized the formation of well-defined crosslinked polymers with various macro structures, topologies and functionality. ${ }^{6-8}$ Cyclic polymers for example have unique properties due to their compact structures. Features such as intrinsic low viscosity, high glass transition temperature ${ }^{9}$, high density, great elasticity ${ }^{10}$, increased circulation and biocompatibility for gene and drug delivery purposes ${ }^{11-12}$ make these crosslinked polymers highly favored.

Aided by the Flory-Stockmayer mean field theory (F-S theory) chemists are provided more control over the polymerization reaction by being able to predict the gel point. ${ }^{13-15}$ The F$\mathrm{S}$ theory predicts gelation at low conversion due to intermolecular crosslinking. ${ }^{16-18}$ As this is highly inconvenient chemists have found ways around this issue, for example by means of chain transfer agents (CTA). ${ }^{19-21}$ Others focused on kinetically controlling the reaction and promoting intramolecular crosslinking while suppressing intermolecular crosslinking. In this way gelation is delayed significantly and control over the polymer architecture is achieved. Wenxing Wang and co-workers have studied this method extensively for homopolymerizations. ${ }^{22-24}$ Sherrington and Armes combined methyl methacrylate (MMA) with a low concentration of ethylene glycol dimethacrylate (EGDMA) in a Cu-based ATRP reaction and utilizing CTAs to gain control over branched polymer synthesis. ${ }^{25}$ Wang in turn improved this methodology by removing the limitation on multifunctional vinyl monomer concentration through precise control over the competition between chain growth and reversible chain termination. ${ }^{26}$ By keeping the growing chains in rapid equilibrium between active and dormant states the polymer growth rate is decreased 
significantly, granting control over the intermolecular crosslinking. Armes et al. applied a similar technique to produce highly branched polymers in a RAFT copolymerization. ${ }^{27}$ Likewise, Taton and coworkers synthesized water-soluble nanogels in a one-pot reaction containing up to 10 mol\% $N, N^{\prime}$-methylenebisacrylamide (MBA). ${ }^{14}$

In 2005 Zhu and Wang were the first to synthesize nanoscale crosslinked polymeric networks (nanogels) with homogenous structures and control over the molecular weight. ${ }^{15}$ Through means of quick initiation and rapid reversible deactivation the amount of growing polymer chains are kept nearly constant. ${ }^{13}$ In combination with dilute conditions and a thermodynamically good solvent, homogenous nanogel structures are produced. ${ }^{28}$ Nanogels are a unique subclass of spherical crosslinked polymers that have intrinsic shrinking and swelling abilities, making them very useful in biomedical applications. ${ }^{29-31}$ Nanogels can be made redox-, enzyme-, $\mathrm{pH}-$, light- or temperatureresponsive making them appealing as drug, protein and gene delivery vehicles or as bioimaging agents. ${ }^{32}$ They also possess a high loading capacity, high water content, desirable mechanical features, large surface area, interior matrix for encapsulation and an average elastic modulus between 0.1 and $100 \mathrm{kPa} .{ }^{33-34}$ Many features such as the surface charge, size, crosslink density, porosity, amphiphilicity and degradability are tunable. As such functional nanogels can be found in gene delivery ${ }^{35-39}$, sensing ${ }^{40-48}$, tissue engineering ${ }^{49-51}$ and anti-fouling ${ }^{52-53}$.

In the field of gene therapy, gene expression is purposefully adjusted to treat genetic diseases. Exogenous nucleic acids such as plasmid DNA, mRNA, small interfering RNA or microRNA are introduced to hamper or stimulate the expression of certain genes. ${ }^{54}$ This genetic material can be directly introduced into the human body. However, nucleotides are negatively charged resulting in low cellular uptake. Additionally, nucleic acids have a short half-life in blood due to their size and the resulting renal clearance. ${ }^{55-56} \mathrm{In}$ order to overcome these limitations, carrier systems coined vectors were developed. Both viral 
and non-viral vectors have found application in gene delivery. Approximately $70 \%$ of vectors used in clinical trials were developed from modified viruses. ${ }^{57-58}$ Although popular, viral vectors present a set of challenges, such as virus production ${ }^{59}$, loading capacity ${ }^{60}$, carcinogenesis ${ }^{61}$ and immunogenicity ${ }^{62}$ - which are absent for non-viral vectors. ${ }^{63-64}$ Lipids and polymers have proven to be efficient non-viral vectors; with many entering clinical trials. ${ }^{65}$

\subsection{Aim and outline}

In this thesis we aim to synthesize novel nanogels by means of a RAFT copolymerization with a monovinyl monomer and a divinyl crosslinker to serve in vitro as a polymeric, nonviral gene vector. In Chapter 2 we update the reader on recent efforts and developments in the surface functionalization of nanogels for biomedical applications; emphasizing internalization of nanogels in cells. In Chapter 3 we apply controlled/living crosslinking polymerization techniques to form size-controlled nanogels serving as versatile platforms that allow post-synthesis modifications. Such useful nanogels include delivery vehicles for gene transfection. In Chapter 4 we synthesize sulfonium nanogels from an epoxide containing precursor to serve as a gene carrier. The grand majority of gene vectors are based on ammonium carrying the positive charge. Although high transfection efficiencies are achieved with current gene vectors, biocompatibility remains an issue. To broaden the scope and potentially lower cytotoxicity, the choice is made to use sulfonium as the cationic functional group as others have obtained great results with this moiety. ${ }^{66}$ To achieve various cationic charges, predetermined amounts of epoxides are functionalized. The remaining epoxides are endcapped to lower toxicity and increase the general biocompatibility of the nanogels. In Chapter 5 the sulfonium nanogels are complexed with plasmid DNA. The binding affinities, sizes and surface charges of the resulting polyplexes 
are discussed. In Chapter 6 we evaluate the sulfonium polyplexes and their transfection efficiency on various cell lines. Various conditions such as incubation time and plasmid DNA concentration per well are tested and optimized. The influence of the polymer macrostructure is also probed by comparing sulfonium nanogels with sulfonium polymers. Finally, in Chapter 7 we reflect on the research conducted in previous chapters and discuss the findings.

\subsection{References}

1. SZWARC, M., 'Living'polymers. Nature 1956, 178 (4543), 1168-1169.

2. Patten, T. E.; Xia, J.; Abernathy, T.; Matyjaszewski, K., Polymers with very low polydispersities from atom transfer radical polymerization. Science 1996, 272 (5263), 866-868.

3. Hawker, C. J.; Bosman, A. W.; Harth, E., New polymer synthesis by nitroxide mediated living radical polymerizations. Chemical reviews 2001, 101 (12), 36613688.

4. YK, C. J. C.; Ercole, F., Living free-radical polymerization by reversible additionfragmentation chain transfer: the RAFT process. Macromolecules 1998, 31, 55595562.

5. Wang, J.-S.; Matyjaszewski, K., Controlled/" living" radical polymerization. Atom transfer radical polymerization in the presence of transition-metal complexes. Journal of the American Chemical Society 1995, 117 (20), 5614-5615.

6. Chen, Q.; Cao, X.; Xu, Y.; An, Z., Emerging Synthetic Strategies for Core CrossLinked Star (CCS) Polymers and Applications as Interfacial Stabilizers: Bridging Linear Polymers and Nanoparticles. Macromolecular rapid communications 2013, 34 (19), 1507-1517. 
7. Frechet, J. M.; Henmi, M.; Gitsov, I.; Aoshima, S.; Leduc, M. R.; Grubbs, R. B., Selfcondensing vinyl polymerization: an approach to dendritic materials. Science 1995, 269 (5227), 1080-1083.

8. Percec, V.; Ahn, C.-H.; Ungar, G.; Yeardley, D.; Möller, M.; Sheiko, S., Controlling polymer shape through the self-assembly of dendritic side-groups. Nature 1998, 391 (6663), 161-164.

9. Zhang, K.; Lackey, M. A.; Cui, J.; Tew, G. N., Gels based on cyclic polymers. Journal of the American Chemical Society 2011, 133 (11), 4140-4148.

10. Wei, H.; Chu, D. S.; Zhao, J.; Pahang, J. A.; Pun, S. H., Synthesis and evaluation of cyclic cationic polymers for nucleic acid delivery. ACS macro letters 2013, 2 (12), 1047-1050.

11. Nasongkla, N.; Chen, B.; Macaraeg, N.; Fox, M. E.; Fréchet, J. M.; Szoka, F. C., Dependence of pharmacokinetics and biodistribution on polymer architecture: effect of cyclic versus linear polymers. Journal of the American Chemical Society 2009, 131 (11), 3842-3843.

12. Chen, B.; Jerger, K.; Fréchet, J. M.; Szoka Jr, F. C., The influence of polymer topology on pharmacokinetics: differences between cyclic and linear PEGylated poly (acrylic acid) comb polymers. Journal of Controlled Release 2009, 140 (3), 203-209.

13. Gao, H.; Matyjaszewski, K., Synthesis of functional polymers with controlled architecture by CRP of monomers in the presence of cross-linkers: From stars to gels. Progress in Polymer Science 2009, 34 (4), 317-350.

14. Taton, D.; Baussard, J.-F.; Dupayage, L.; Poly, J.; Gnanou, Y.; Ponsinet, V.; Destarac, M.; Mignaud, C.; Pitois, C., Water soluble polymeric nanogels by xanthatemediated radical crosslinking copolymerisation. Chemical communications 2006, (18), 1953-1955. 
15. Wang, A. R.; Zhu, S., Branching and gelation in atom transfer radical polymerization of methyl methacrylate and ethylene glycol dimethacrylate. Polymer Engineering \& Science 2005, 45 (5), 720-727.

16. PJ, F., Molecular size distribution in three dimensional polymers. i. gelation 1. J Am Chem Soc 1941, 63 (11), 3083-3090.

17. Stockmayer, W. H., Theory of molecular size distribution and gel formation in branched-chain polymers. The Journal of chemical physics 1943, 11 (2), 45-55.

18. Stockmayer, W. H., Theory of molecular size distribution and gel formation in branched polymers II. General cross linking. The Journal of Chemical Physics 1944, 12 (4), 125-131.

19. Baudry, R.; Sherrington, D., Synthesis of highly branched poly (methyl methacrylate) s using the "strathclyde methodology" in aqueous emulsion. Macromolecules 2006, 39 (4), 1455-1460.

20. Costello, P.; Martin, I.; Slark, A.; Sherrington, D.; Titterton, A., Branched methacrylate copolymers from multifunctional monomers: chemical composition and physical architecture distributions. Polymer 2002, 43 (2), 245-254.

21. O'brien, N.; McKee, A.; Sherrington, D.; Slark, A.; Titterton, A., Facile, versatile and cost effective route to branched vinyl polymers. Polymer 2000, 41 (15), 60276031.

22. Gao, Y.; Zhou, D.; Zhao, T.; Wei, X.; McMahon, S.; O'Keeffe Ahern, J.; Wang, W.; Greiser, U.; Rodriguez, B. J.; Wang, W., Intramolecular cyclization dominating homopolymerization of multivinyl monomers toward single-chain cyclized/knotted polymeric nanoparticles. Macromolecules 2015, 48 (19), 68826889.

23. Zheng, Y.; Newland, B.; Tai, H.; Pandit, A.; Wang, W., Single cyclized molecule structures from RAFT homopolymerization of multi-vinyl monomers. Chemical Communications 2012, 48 (25), 3085-3087. 
24. Gao, Y.; Newland, B.; Zhou, D.; Matyjaszewski, K.; Wang, W., Controlled Polymerization of Multivinyl Monomers: Formation of Cyclized/Knotted SingleChain Polymer Architectures. Angewandte Chemie International Edition 2017, 56 (2), 450-460.

25. Isaure, F.; Cormack, P. A.; Graham, S.; Sherrington, D. C.; Armes, S. P.; Bütün, V., Synthesis of branched poly (methyl methacrylate) s via controlled/living polymerisations exploiting ethylene glycol dimethacrylate as branching agent. Chemical communications 2004, (9), 1138-1139.

26. Wang, W.; Zheng, Y.; Roberts, E.; Duxbury, C. J.; Ding, L.; Irvine, D. J.; Howdle, S. M., Controlling chain growth: a new strategy to hyperbranched materials. Macromolecules 2007, 40 (20), 7184-7194.

27. Rosselgong, J.; Armes, S. P.; Barton, W.; Price, D., Synthesis of highly branched methacrylic copolymers: observation of near-ideal behavior using RAFT polymerization. Macromolecules 2009, 42 (16), 5919-5924.

28. Kurochkin, S.; Grachev, V., Reversible deactivation radical polymerization of polyfunctional monomers. Polymer Science Series C 2015, 57 (1), 20-31.

29. Sanson, N.; Rieger, J., Synthesis of nanogels/microgels by conventional and controlled radical crosslinking copolymerization. Polymer Chemistry 2010, 1 (7), 965-977.

30. Sharma, A.; Garg, T.; Aman, A.; Panchal, K.; Sharma, R.; Kumar, S.; Markandeywar, T., Nanogel-an advanced drug delivery tool: Current and future. Artificial cells, nanomedicine, and biotechnology 2016, 44 (1), 165-177.

31. Hernández-Adame, L.; Angulo, C.; García-Silva, I.; Palestino, G.; Rosales-Mendoza, S., An overview of nanogel-based vaccines. Expert review of vaccines 2019, 18 (9), 951-968. 
32. Ekkelenkamp, A. E.; Elzes, M. R.; Engbersen, J. F.; Paulusse, J. M., Responsive crosslinked polymer nanogels for imaging and therapeutics delivery. Journal of Materials Chemistry B 2018, 6 (2), 210-235.

33. Oh, J. K.; Lee, D. I.; Park, J. M., Biopolymer-based microgels/nanogels for drug delivery applications. Progress in polymer science 2009, 34 (12), 1261-1282.

34. Oh, J. K.; Drumright, R.; Siegwart, D. J.; Matyjaszewski, K., The development of microgels/nanogels for drug delivery applications. Progress in Polymer Science 2008, 33 (4), 448-477.

35. Dimde, M.; Neumann, F.; Reisbeck, F.; Ehrmann, S.; Cuellar-Camacho, J. L.; Steinhilber, D.; Ma, N.; Haag, R., Defined pH-sensitive nanogels as gene delivery platform for siRNA mediated in vitro gene silencing. Biomaterials science 2017, 5 (11), 2328-2336.

36. Hong, C. A.; Kim, J. S.; Lee, S. H.; Kong, W. H.; Park, T. G.; Mok, H.; Nam, Y. S., Reductively Dissociable siRNA-Polymer Hybrid Nanogels for Efficient Targeted Gene Silencing. Advanced Functional Materials 2013, 23 (3), 316-322.

37. Mauri, E.; Perale, G.; Rossi, F., Nanogel functionalization: a versatile approach to meet the challenges of drug and gene delivery. ACS Applied Nano Materials 2018, $1(12), 6525-6541$.

38. Park, J. S.; Yi, S. W.; Kim, H. J.; Kim, S. M.; Shim, S. H.; Park, K.-H., Sunflower-type nanogels carrying a quantum dot nanoprobe for both superior gene delivery efficacy and tracing of human mesenchymal stem cells. Biomaterials 2016, 77, 1425.

39. Park, J. S.; Yi, S. W.; Kim, H. J.; Park, K.-H., Receptor-mediated gene delivery into human mesenchymal stem cells using hyaluronic acid-shielded polyethylenimine/pDNA nanogels. Carbohydrate polymers 2016, 136, 791-802. 
40. Wu, W.; Shen, J.; Banerjee, P.; Zhou, S., Core-shell hybrid nanogels for integration of optical temperature-sensing, targeted tumor cell imaging, and combined chemo-photothermal treatment. Biomaterials 2010, 31 (29), 7555-7566.

41. Wu, W.; Shen, J.; Li, Y.; Zhu, H.; Banerjee, P.; Zhou, S., Specific glucose-to-SPR signal transduction at physiological $\mathrm{pH}$ by molecularly imprinted responsive hybrid microgels. Biomaterials 2012, 33 (29), 7115-7125.

42. Wu, W.; Mitra, N.; Yan, E. C.; Zhou, S., Multifunctional hybrid nanogel for integration of optical glucose sensing and self-regulated insulin release at physiological pH. Acs Nano 2010, 4 (8), 4831-4839.

43. Li, C.; Liu, S., Responsive nanogel-based dual fluorescent sensors for temperature and $\mathrm{Hg} 2+$ ions with enhanced detection sensitivity. Journal of Materials Chemistry 2010, 20 (47), 10716-10723.

44. Zhu, H.; Li, Y.; Qiu, R.; Shi, L.; Wu, W.; Zhou, S., Responsive fluorescent Bi2O3@ PVA hybrid nanogels for temperature-sensing, dual-modal imaging, and drug delivery. Biomaterials 2012, 33 (10), 3058-3069.

45. Wu, W.; Shen, J.; Gai, Z.; Hong, K.; Banerjee, P.; Zhou, S., Multi-functional coreshell hybrid nanogels for $\mathrm{pH}$-dependent magnetic manipulation, fluorescent $\mathrm{pH}$ sensing, and drug delivery. Biomaterials 2011, 32 (36), 9876-9887.

46. Peng, H. S.; Stolwijk, J. A.; Sun, L. N.; Wegener, J.; Wolfbeis, O. S., A nanogel for ratiometric fluorescent sensing of intracellular $\mathrm{pH}$ values. Angewandte Chemie 2010, 122 (25), 4342-4345.

47. Wu, W.; Shen, J.; Banerjee, P.; Zhou, S., Chitosan-based responsive hybrid nanogels for integration of optical $\mathrm{pH}$-sensing, tumor cell imaging and controlled drug delivery. Biomaterials 2010, 31 (32), 8371-8381.

48. Wu, W.; Aiello, M.; Zhou, T.; Berliner, A.; Banerjee, P.; Zhou, S., In-situ immobilization of quantum dots in polysaccharide-based nanogels for integration 
of optical pH-sensing, tumor cell imaging, and drug delivery. Biomaterials 2010, 31 (11), 3023-3031.

49. Fujioka-Kobayashi, M.; Ota, M. S.; Shimoda, A.; Nakahama, K.-i.; Akiyoshi, K.; Miyamoto, Y.; Iseki, S., Cholesteryl group-and acryloyl group-bearing pullulan nanogel to deliver BMP2 and FGF18 for bone tissue engineering. Biomaterials 2012, 33 (30), 7613-7620.

50. Steinhilber, D.; Rossow, T.; Wedepohl, S.; Paulus, F.; Seiffert, S.; Haag, R., A microgel construction kit for bioorthogonal encapsulation and $\mathrm{pH}$-controlled release of living cells. Angewandte Chemie International Edition 2013, 52 (51), 13538-13543.

51. Xia, Y.; He, X.; Cao, M.; Chen, C.; Xu, H.; Pan, F.; Lu, J. R., Thermoresponsive microgel films for harvesting cells and cell sheets. Biomacromolecules 2013, 14 (10), 3615-3625.

52. Zhao, C.; Chen, Q.; Patel, K.; Li, L.; Li, X.; Wang, Q.; Zhang, G.; Zheng, J., Synthesis and characterization of $\mathrm{pH}$-sensitive poly ( $\mathrm{N}$-2-hydroxyethyl acrylamide)-acrylic acid (poly (HEAA/AA)) nanogels with antifouling protection for controlled release. Soft Matter 2012, 8 (30), 7848-7857.

53. Bridges, A. W.; Singh, N.; Burns, K. L.; Babensee, J. E.; Lyon, L. A.; García, A. J., Reduced acute inflammatory responses to microgel conformal coatings. Biomaterials 2008, 29 (35), 4605-4615.

54. Luo, D.; Saltzman, W. M., Synthetic DNA delivery systems. Nature biotechnology 2000, 18 (1), 33-37.

55. Gao, S.; Dagnaes-Hansen, F.; Nielsen, E. J. B.; Wengel, J.; Besenbacher, F.; Howard, K. A.; Kjems, J., The effect of chemical modification and nanoparticle formulation on stability and biodistribution of siRNA in mice. Molecular therapy 2009, 17 (7), 1225-1233. 
56. Naeye, B.; Deschout, H.; Caveliers, V.; Descamps, B.; Braeckmans, K.; Vanhove, C.; Demeester, J.; Lahoutte, T.; De Smedt, S. C.; Raemdonck, K., In vivo disassembly of IV administered siRNA matrix nanoparticles at the renal filtration barrier. Biomaterials 2013, 34 (9), 2350-2358.

57. Kay, M. A., State-of-the-art gene-based therapies: the road ahead. Nature Reviews Genetics 2011, 12 (5), 316-328.

58. Mingozzi, F.; High, K. A., Therapeutic in vivo gene transfer for genetic disease using AAV: progress and challenges. Nature reviews genetics 2011, 12 (5), 341-355.

59. Bouard, D.; Alazard-Dany, N.; Cosset, F. L., Viral vectors: from virology to transgene expression. British journal of pharmacology 2009, 157 (2), 153-165.

60. Thomas, C. E.; Ehrhardt, A.; Kay, M. A., Progress and problems with the use of viral vectors for gene therapy. Nature Reviews Genetics 2003, 4 (5), 346-358.

61. Baum, C.; Kustikova, O.; Modlich, U.; Li, Z.; Fehse, B., Mutagenesis and oncogenesis by chromosomal insertion of gene transfer vectors. Human gene therapy 2006, 17 (3), 253-263.

62. Bessis, N.; GarciaCozar, F.; Boissier, M., Immune responses to gene therapy vectors: influence on vector function and effector mechanisms. Gene therapy 2004, 11 (1), S10-S17.

63. Pack, D. W.; Hoffman, A. S.; Pun, S.; Stayton, P. S., Design and development of polymers for gene delivery. Nature reviews Drug discovery 2005, 4 (7), 581-593.

64. Mintzer, M. A.; Simanek, E. E., Nonviral vectors for gene delivery. Chemical reviews 2009, 109 (2), 259-302.

65. Yin, H.; Kanasty, R. L.; Eltoukhy, A. A.; Vegas, A. J.; Dorkin, J. R.; Anderson, D. G., Non-viral vectors for gene-based therapy. Nature Reviews Genetics 2014, 15 (8), 541-555. 
66. Zhu, D.; Yan, H.; Liu, X.; Xiang, J.; Zhou, Z.; Tang, J.; Liu, X.; Shen, Y., Intracellularly disintegratable polysulfoniums for efficient gene delivery. Advanced Functional Materials 2017, 27 (16), 1606826. 
Chapter 1

14 I $P$ a g e 


\section{Chapter 2}

\section{Surface functionalizations and cellular uptake of nanogels in biomedical applications}
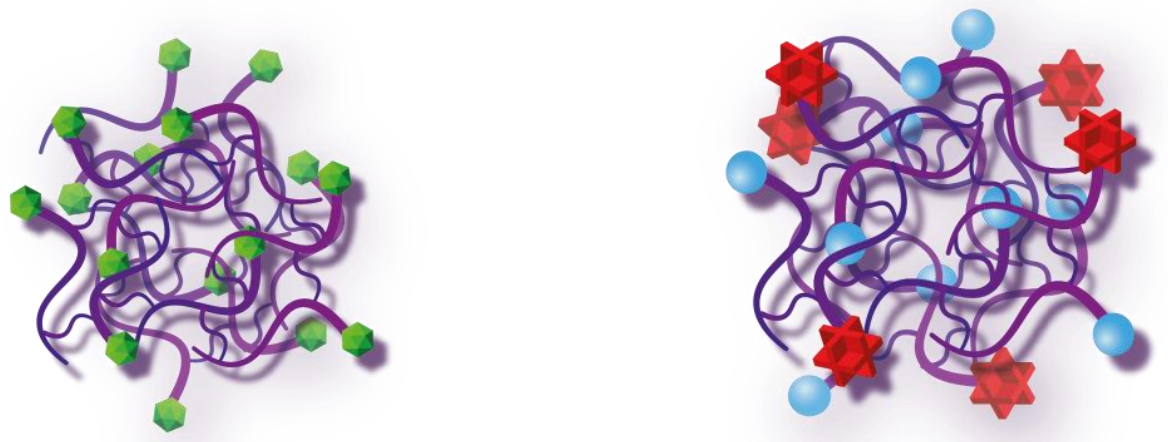


\subsection{Introduction}

Drug delivery systems amplify drug efficacy by limiting its possible toxicity and improving its pharmacokinetics by enhancing the drug release profile, distribution, absorption, eventual degradation and elimination of the drug. ${ }^{1}$ Micelles, block copolymers, dendrimers, liposomes and hydrogels can all serve as polymeric drug carriers. ${ }^{2-4}$ Many, however, never make it to clinical trials due to poor properties such as low biodistribution, low stability, rapid clearance and high-toxicity. ${ }^{5}$ Nanogels are three-dimensional crosslinked polymer networks with nanometer dimensions that are widely used in (nano)medicine due to their favorable properties. ${ }^{6}$ Intrinsic swelling and shrinking upon exposure to stimuli, large surface area, high water content, high payload capacity, sitespecific delivery, versatility in design, an inner network for the encapsulation, protection and controlled release of molecular therapeutics, as depicted in Scheme 1, are some of the many features that make nanogels very attractive for biomedical applications. ${ }^{7-11}$ Unlike hydrogels, nanogels are not limited by their rate of diffusion in stimuli-responsive applications. ${ }^{12}$ As nanogels are smaller than hydrogels, their diffusion path length is also decreased. Functional nanogels can be found in tissue engineering, ${ }^{8,}{ }^{13-14}$ cancer treatment, ${ }^{15-18}$ bioimaging, ${ }^{19-20}$ pain management, ${ }^{21}$ antifouling, ${ }^{22-23}$ ophthalmic diseases ${ }^{24}$ and biosensing $20,25-31$. In order to fully utilize the potential of nanogels they need to be tailor made to the specific needs of the field of interest. The size, surface charge, stimulus response and surface chemistry are all responsible for the in vitro and in vivo behavior of nanogels. ${ }^{32-35}$ Ligand-decorated nanogels for instance will actively target the nanogels to the location of the corresponding receptor. ${ }^{36-37}$

Previous reviews have focused on the synthesis, ${ }^{38}$ applications $^{39}$ and degradability ${ }^{40-41}$ of nanogels. In this chapter we will direct our attention to the surface functionalization of nanogels and their cellular uptake. 


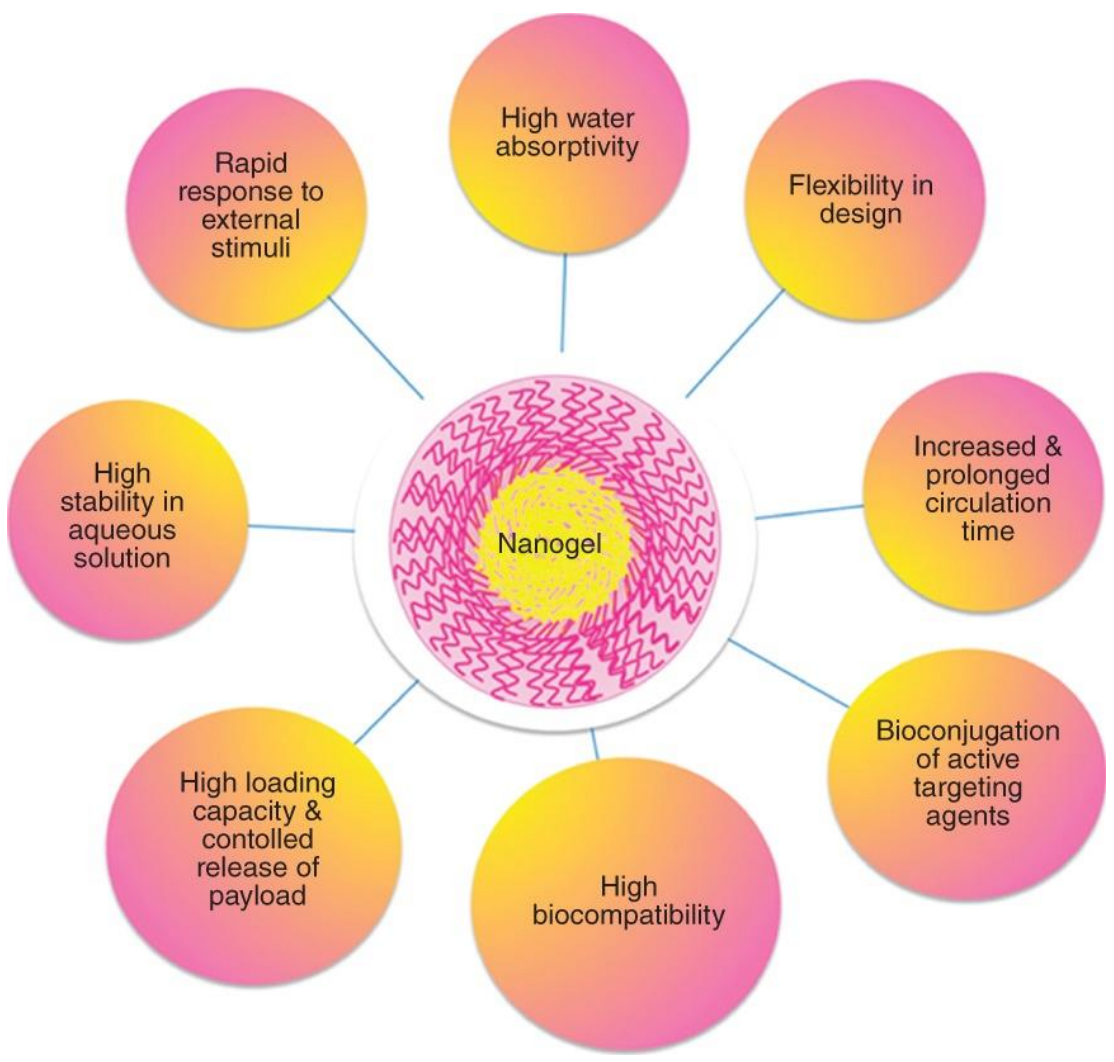

Scheme 1. General characteristic features of nanogels. ${ }^{42}$ Reproduced with permission from John Wiley and Sons.

\subsection{Functional nanogels through surface modification}

Once therapeutic drugs have been manufactured that successfully treat illnesses, they still face challenges such as low water-solubility ${ }^{43}$, early release ${ }^{44}$, high toxicity ${ }^{45}$, low stability ${ }^{45}$ and/or limited uptake ${ }^{46}$. Delivery platforms are able to overcome these obstacles by protecting, delivering and releasing the drug at its intended target. Nanogels make useful particles for delivery and protection of small molecules in various fields of (nano)medicine. In order to achieve high efficacy of these functional nanogels; they are often tailor-made to suit the needs of the application. Nanogels for cancer cellular uptake 
for instance, require a size between 10 and $200 \mathrm{~nm}$ to benefit from the enhanced permeability and retention effect (EPR)..$^{47-48}$ Nanogels meant to function as nucleic acid vectors often bare cationic charges to securely bind negatively-charged DNA or RNA. ${ }^{49-51}$ Modifying nanogels by surface conjugation with small molecules of interest such as carbon dots $^{52}$ and dyes ${ }^{53-55}$ enhances their utility towards various biological applications. Toxic moieties on nanogels can also be removed by means of post-polymerization modification. ${ }^{56}$

\subsection{Nanogel surface functionalization with PEG}

The most common surface modification in nanomedicine is PEGylation. PEG chains increase biocompatibility, hydrophilicity, antifouling properties and the biodistribution of nanomaterials. ${ }^{57-58}$ Simple modifications such as PEGylation improve the therapeutic effect, tunability and biocompatibility of functional nanogels. ${ }^{56}$ Mauri et al. grafted mPEG chains with carboxyl or imidazole terminal groups to PEG-PEI nanogels as seen in Scheme $2 .^{59}$ The carboxyl group reacts with the matrix forming an amide bond (NG-COOH). The PEG chain containing an imidazole end group coated the nanogel with PEG chains through NHS/EDC coupling, utilizing imidazole as a leaving group and forming NG-Im. 


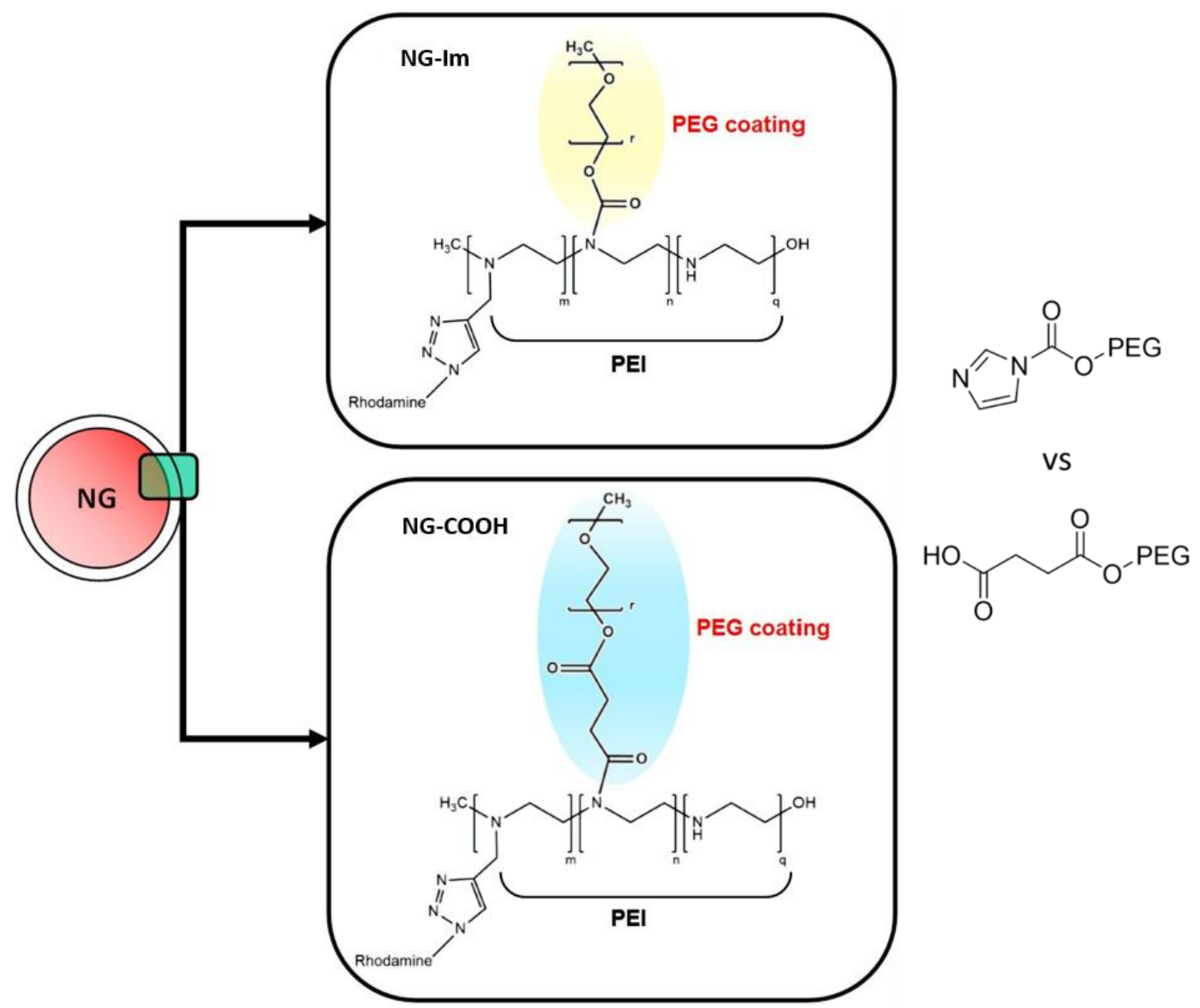

Scheme 2. PEG-PEI nanogels were surface modified with a PEG coating, containing either a carboxylate end functionality forming NG-COOH, or an imidazole end group serving as a leaving group, affording NG-Im. ${ }^{59}$ Reproduced and adapted with permission from Elsevier.

As a result of the different coating methods, NG-Im contained half as many PEG chains on its surface compared to NG-COOH, as determined by ${ }^{1} \mathrm{H}$ NMR. Both nanogels were shown to be non-toxic to microglia cells, a type of macrophages, even after 3 days of incubation. However, the nanogels exhibited different rates for cellular uptake in microglia cells. NG$\mathrm{COOH}$ was taken up less than uncoated nanogel, whereas the internalization of NG-Im was significantly increased. This is most likely due to the thicker PEG layer present on NG- 
$\mathrm{COOH}$, hindering proper interaction between the cationic charge of the PEI units and the microglia cell membrane. The thin layer of PEG coating on NG-Im provided enough stability to increase uptake compared to "bare" nanogels without hindering the interactions with the microglia cell membrane. The authors also studied the effect of the PEGylation on drug delivery by means of adsorption in the external layer of the nanogel matrix. ${ }^{60}$ Fluorescein anion (SF) and neutral Rhodamine $B(\mathrm{RhB})$ where utilized as drug mimetics of roughly the same dimensions. NG-Im had the highest loading capacity for both SF and RhB, compared to the bare nanogel and NG-COOH; most likely due to its larger diameter. Release studies displayed an initial burst followed by sustained release over a period of 15 days, for both SF and RhB. The release profiles were similar for all nanogels, however NG-Im displayed a lower rate for both burst and sustained release. The diffusion coefficient of NG-Im was an order of magnitude lower than for NG-COOH and bare nanogel. Throughout the study there was no difference between values obtained for SF and RhB, indicating that the PEG thickness of the NGs - and not their ionic charges - influenced the diffusion coefficient.

Wang and coworkers similarly utilized carbodiimide coupling to attach PEG chains with a folate functionality to nanogels. ${ }^{61}$ Folate receptors are overexpressed on the cell membranes of tumor cells but not on healthy cells. ${ }^{62}$ This feature enables active targeting of nano-therapeutics to cancer cells. ${ }^{63-67}$ The PEG chains render the nanogels less susceptible to renal clearance and mitigates unspecific binding. ${ }^{57-58}$ The authors first synthesized zinc dimethacrylate (ZDMA) to function as a crosslinker. In a next step methacrylic acid (MAA), AIBN and ZDMA were reacted to a nanogel by means of refluxprecipitation polymerization, forming zinc-crosslinked nanogels (ZCLNs). Subsequently, the nanogels were surface functionalized with $\mathrm{NH}_{2}$-PEG-folate by means of EDC/NHS chemistry, affording folate-PEG conjugated zinc-crosslinked nanogels (FPZCLNs). Utilizing zinc chelation as a means of crosslinking is a creative way to ensure intracellular 
biodegradability of the nanogels even at regular GSH concentrations - which is impossible for disulfide bonds. Zinc (II) can chelate with the two thiols of GSH and form stable bonds; as witnessed in zinc finger proteins in the human body. ${ }^{68-69}$ The nanogels were loaded with doxorubicin (DOX) which were held in place by means of electrostatic interactions. At mildly acidic $\mathrm{pH}$ the carboxylate ion of MAA would be protonated, resulting in cleavage of the $\mathrm{Zn}^{2+}-\mathrm{O}$ bond, followed by expulsion of DOX from the nanogel. These FPZCLNs are thus $\mathrm{pH}$ - and GSH-responsive as seen in Scheme 3.
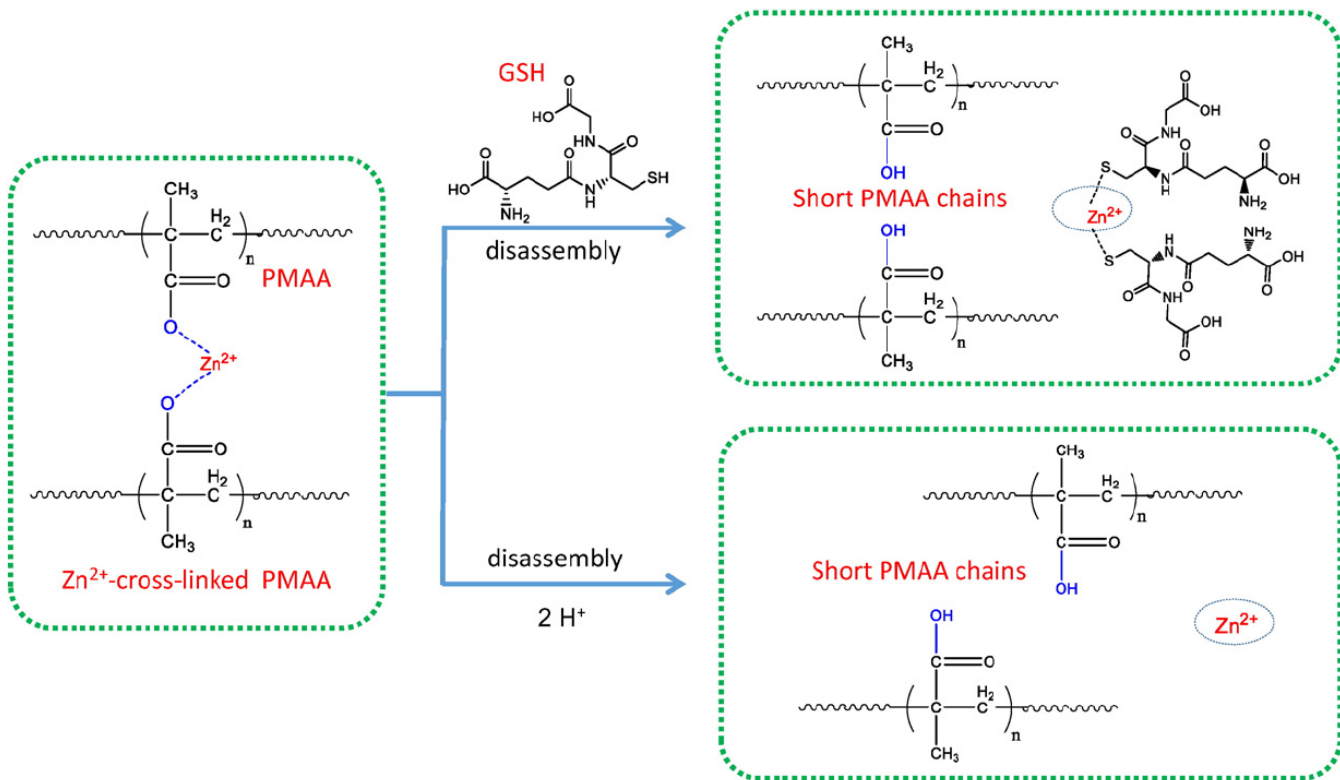

Scheme 3. The nanogels are crosslinked by $\mathrm{Zn}^{2+}$ chelation with oxygen. At acidic $\mathrm{pH}$ and/or in the presence of $\mathrm{GSH}$, these bonds are broken and replaced by free $\mathrm{Zn}^{2+}$ ions or $\mathrm{Zn}$-chelated GSH dimers. ${ }^{61}$ Reproduced with permission from Elsevier.

An increase in crosslink density meant fewer free carboxylate ions and thus a lower loading capacity of the nanogels. Cytotoxicity studies with healthy HEK293 cells and DOXfree FPZCLNs exhibited high cell viability up to $200 \mu \mathrm{g} / \mathrm{mL}$; indicating that the polymer 
carrier itself is not toxic to healthy cells. Cancerous HeLa cells were treated with various concentrations of free DOX, DOX-loaded PZCLNs (absent of folate) and DOX-loaded FPZCLNs. A dose-dependent cytotoxicity trend was apparent - with targeted, DOX-loaded FPZCLNs and free DOX displaying the highest cytotoxicity. Minimal DOX leaked from FPZCLNs at physiological conditions indicating that its therapeutic effect is significantly higher than free DOX, as DOX-loaded FPZCLNs would release its cargo at the target site and not leak DOX prematurely. Dosing with an equal amount of free-DOX would result in harm to healthy cells and lower therapeutic effect at tumor cells. Both FPZCLNs and PZCLNs were able to enter HeLa cells and release DOX in the cytoplasm. However, FPZCLNs achieved a 2.4 times higher concentration of DOX in the cell nucleus than PZCLNs demonstrating folate ligand-mediated endocytosis.

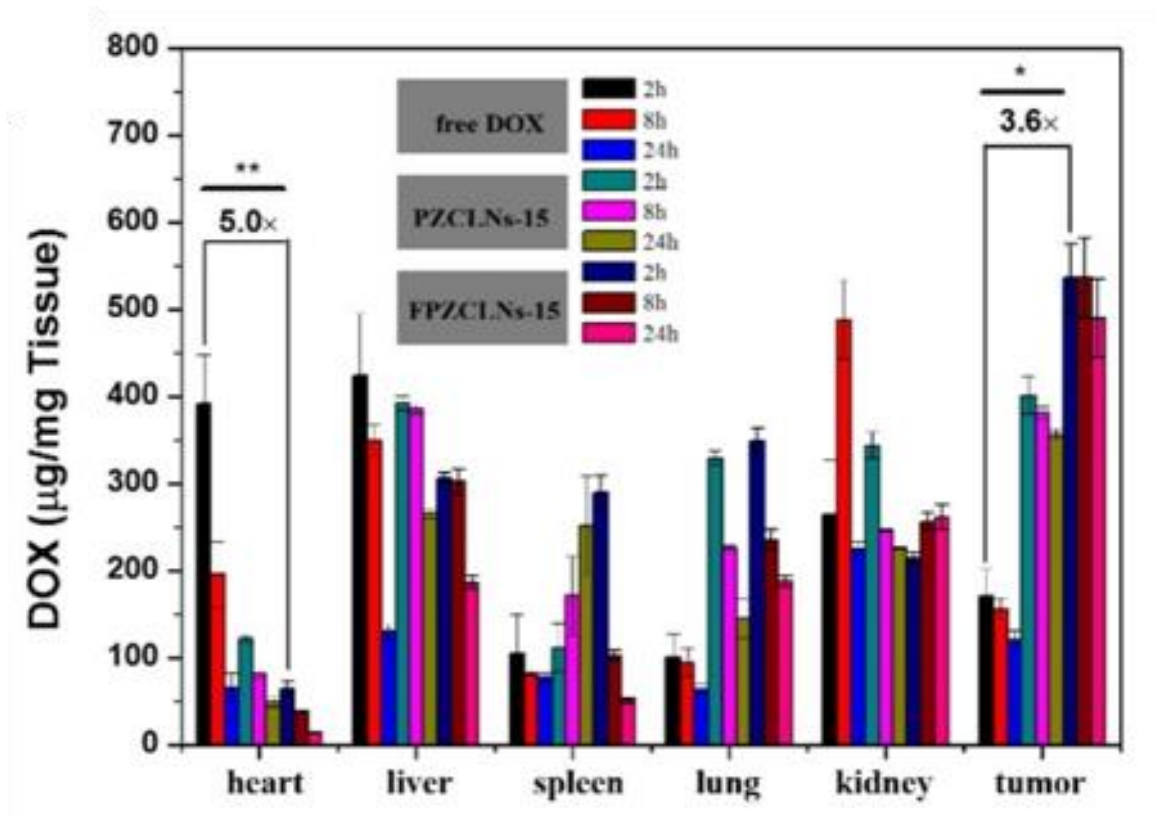

Figure 1. Mice bearing $\mathrm{H} 22$ tumors were treated with free DOX, PZCLNs-15 and FPZCLNs-15; with 15 denoting the crosslink density. Free DOX was present in large quantities in the heart and liver after $2 \mathrm{~h}$. Contrarily, treatment with FPZCLNs-15 resulted in 5 times less DOX in the heart and 3.6 times more DOX in the tumor, after $2 \mathrm{~h} .{ }^{61}$ Adapted and reproduced with permission from Elsevier. 
H22 tumor-bearing mice were injected with free DOX, DOX-loaded PZCLNs and DOXloaded FPZCLNs in their tail vein. The fluorescence of DOX in their organs were analyzed after $2 \mathrm{~h}, 8 \mathrm{~h}$ and $24 \mathrm{~h}$ as seen in Figure 1. Free DOX had traveled to all major organs including the heart, lungs, kidneys and the tumor. After $8 \mathrm{~h}$ the majority of the DOX had made its way to the liver and spleen as is expected for foreign objects. ${ }^{70-71}$ DOX-loaded PZCLNs and DOX-loaded FPZCLNs displayed high DOX fluorescence in the tumor plus low and decreasing fluorescence in the liver and spleen. The presence of the PEG corona on the nanogels most likely hinders their clearance by the reticuloendothelial system, providing ample time for the nanogels to accumulate in the tumor. As hypothesized, active targeted FPZCLN outperformed PZCLN. Mice treated with FPZCLNs exhibited the lowest rate of tumor growth.

PEGylation of nanogels can also be achieved by means of click chemistry. Chen, Tezcan et al. synthesized complex biodegradable nanogels for the purpose of overcoming multidrug resistance. Nano therapeutics can overcome multidrug resistance as particles are internalized by cells through endocytosis as opposed to passive diffusion. ${ }^{72-74} \mathrm{~A}$ copolymer consisting of $\mathrm{N}$-(2-azidoethyl) methacrylamide (AzEMAm), hydroxyethyl methacrylamide (HEMAm) and hydroxyethyl methacrylamide-oligoglycolates (HEMAmGly) was subjected to inverse mini-emulsion photopolymerization in the presence of DOX methacrylamide (DOX-MA). Incorporation of DOX-MA into the nanogel backbone through its methacrylamide functionality afforded azide-decorated nanogels bearing pendant DOX from a labile hydrazone link (DOX-NG) as depicted below in Scheme $4 .{ }^{75} \mathrm{Next}$, PEG chains containing an alkyne end group were clicked onto the surface of the DOX-NG (PEGDOX-NG). ${ }^{76-79} A$ fraction of the PEG chains had additional folic acid conjugates at the other chain end (FA-DOX-NG). 


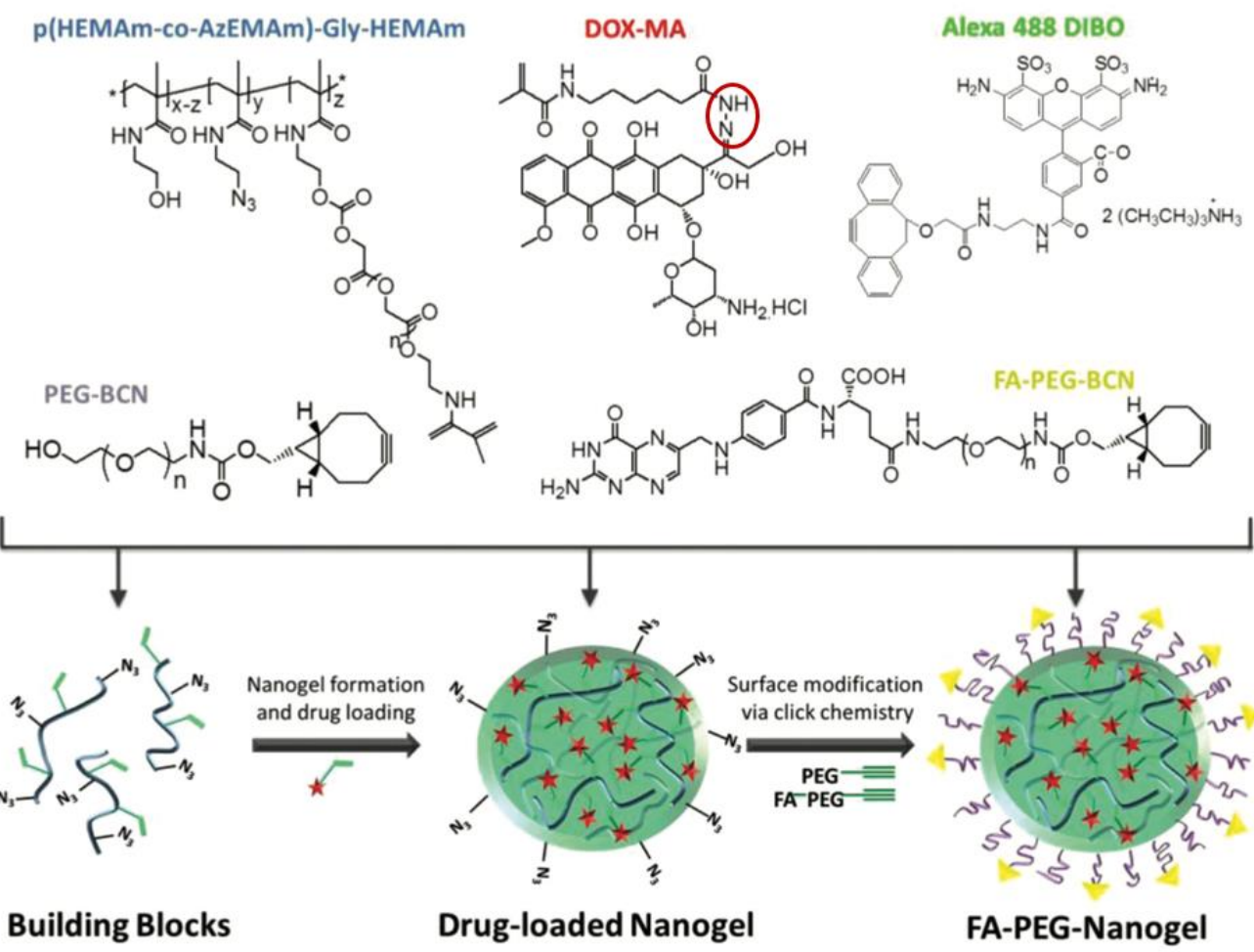

Scheme 4. A schematic overview of the DOX-functionalized nanogels synthesized by Chen, Tezcan et al. The labile hydrazone linking DOX to the nanogel is circled in red. ${ }^{75}$ Reproduced and adapted with permission from Royal Society of Chemistry.

Uptake studies were conducted on B16F10 melanoma cells and A549 lung carcinoma cells. B16F10 cells overexpress the receptor for folate, whereas A549 does not express folate receptors. B16F10 cells exhibited the highest fluorescence when incubated with free DOX, followed by targeted FA-DOX-NG, DOX-NG and PEG-DOX-NG. DOX-MA exhibited no fluorescence. A549 cells likewise exhibited the highest fluorescence for free DOX and the lowest for DOX-MA. However, as A549 has no folate receptors, not much difference was seen between the fluorescence resulting from the uptake of FA-DOX-NG and PEG-DOXNG. FA-DOX-NGs were labeled with Alexa 388 via copper free click chemistry and their 
uptake in B16F10 melanoma cells was imaged and quantified. The Pearson correlation coefficients were calculated after 6 hours of incubation. The highest value was found for nanogel colocalization with endosomes/lysosomes indicating that the majority of the nanogels were in the endosomes/lysosomes. The lowest Pearson correlation coefficient was found for nanogels and cell nuclei meaning the nanogels did not enter the cell nucleus. The coefficients for the colocalization of DOX and nanogel as well as DOX and endosomes/lysosomes were both $<0.5$ suggesting that some DOX were still attached to the nanogels. Additionally, these results confirm the intracellular release of DOX from the nanogels as a result of the hydrolysis of the hydrazone at acidic $\mathrm{pH}$. The colocalization of DOX and nuclei had a Pearson coefficient of $>0.5$ indicating high accumulation of DOX in the nuclei of the B16F10 cells. To validate the uptake pathway of FA-DOX-NGs, competitive inhibition experiments were done with B16F10 cells and A549 cells both in the presence and absence of free folic acid in the medium. For A549 cells no difference was noted in the cell viability of cells incubated with FA-DOX-NGs with or without folic acid. Likewise, incubation of PEG-DOX-NGs with A549 cells in the presence or absence of folic acid had no effect on the cell viability. For B16F10 however, FA-DOX-NGs incubated in the presence of free folic acid had a higher cell viability than those incubated without folic acid present. This is due to competition between FA-DOX-NGs and folic acid for the folate receptors. As a result FA-DOX-NGs binds fewer receptors leading to lower uptake of toxic DOX-releasing nanogels and thus higher cell viability. It can be concluded that FADOX-NGs are taken up by B16F10 cells via a receptor mediated uptake pathway. 4T1 breast cancer cells that are resistant to DOX and overexpress folate receptors were incubated with free DOX, DOX-MA, DOX-NG, PEG-DOX-NG and FA-DOX-NG. As expected cellular uptake was significantly lower compared to incubation with 4T1 DOX sensitive cells, as seen in Figure 2 on the left. 

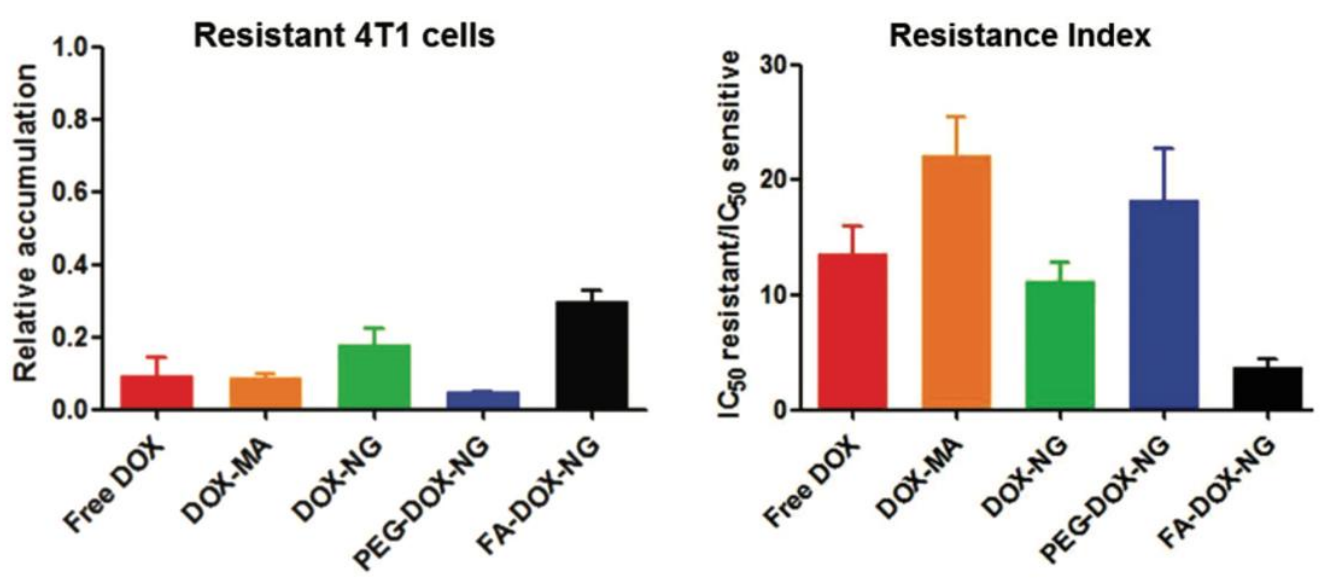

Figure 2. Free DOX, DOX-MA, DOX-NG, PEG-DOX-NG and FA-DOX-NG were incubated with DOX-resistant 4T1 breast cancer cells. Cellular uptake was relatively low. However, FA-DOX-NG was taken up 3 times as much as free DOX owing to its low resistance index. ${ }^{75}$ Reproduced and adapted with permission from Royal Society of Chemistry.

However, FA-DOX-NG displayed 3 times higher DOX uptake than free DOX (Figure 2, right panel), owing to its active targeting of the folate receptors. As such, DOX resistance of the $4 \mathrm{~T} 1$ breast cancer cells was bypassed.

\subsection{Nanogel surface functionalization with peptides}

Surface functionalization of nanogels can be used to achieve multifunctionality and augment their selectivity to (tumor) cells. The group of Sanyal synthesized nanogels by heating thermo-responsive co-polymers above their LCTS achieving self-assembly; followed by controlled crosslinking, in a method first described by Thayumanavan and coworkers. ${ }^{80-81}$ The resulting nanogel had "clickable" maleimide and thiol groups for the conjugation of functional small molecules by means of thiol-ene chemistry (Scheme 5). 


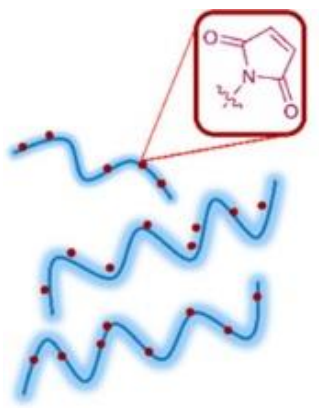

Thermoresponsive Reactive Copolymers

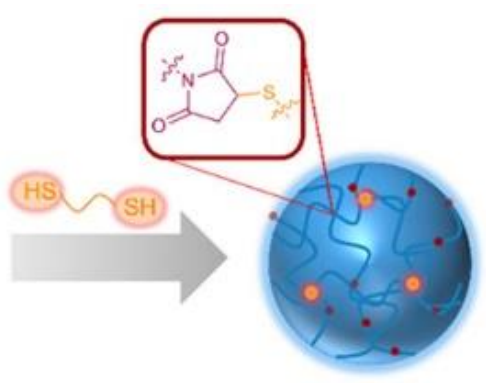

Clickable-Nanogel

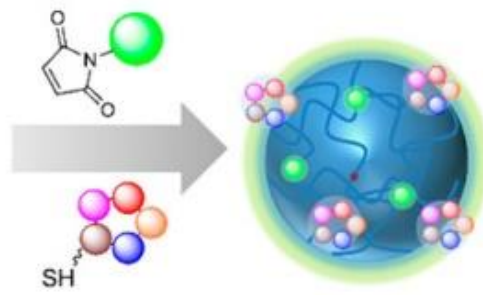

Functional-Nanogel

Scheme 5. Thermo-responsive polymers were self-assembled and crosslinked to a "clickable" nanogel. ${ }^{80}$ Reprinted (adapted) with permission from Ref ${ }^{80}$. Copyright 2017 American Chemical Society.

To form an imaging modality for active targeting of breast cancer cells, cRGDfC peptide and a fluorescein dye were covalently bound to the nanogels. The targeted functional nanogels were non-toxic up to $1 \mathrm{mg} / \mathrm{mL}$ and demonstrated higher uptake than nanogels lacking the targeting peptide as confirmed by fluorescence microscopy and flow cytometry.

The group of Peng and Yang also utilized a cRGD peptide (specifically cRGDfk) to target $\alpha_{v} \beta_{3}$ integrins overexpressed on breast cancer cells. ${ }^{82}$ They synthesized a dualresponsive nanogel from NIPAM and MAA and covalently bound cRGDfk peptide to its surface through EDC/NHS chemistry (CRGD-NG). The carboxylate moieties of MAA were reacted with the amines of the peptide. CRGD-NG experienced higher cellular uptake by MDA-MD-231 cells bearing $\alpha_{v} \beta_{3}$ integrins, than MCF-7 cells that are $\alpha_{v} \beta_{3}$-negative. The ligand-decorated nanogel was then co-loaded with lidocaine and cisplatin (Lido/cRGD-NGPt).$^{83}$ Lidocaine is an inhibitor for voltage-gated $\mathrm{Na}^{+}$channels (VGSC), which promote metastasis by regulating migration and invasion of cancer cells. ${ }^{84}$ Lidocaine also enhances the cytotoxicity of chemotherapy drug cisplatin. ${ }^{85-86}$ Cisplatin was loaded by ligand 
exchange and lidocaine was physically entrapped in the nanogel construct. By combining active targeting, dual-response and multiple therapeutics, the authors aim to treat cancer and alleviate the associated hurdles, such as adverse effects of chemo drugs. In vitro drug release experiments exhibited a cisplatin release rate that was twice as high at $\mathrm{pH}=5.5$ (tumor endosome) than at $\mathrm{pH}=7.38$ (physiological conditions), confirming a $\mathrm{pH}$ response. The synergistic effect of combining cisplatin treatment with lidocaine is displayed in Figure 3. Higher cytotoxicity was achieved for cells treated with Lido/cRGD-NG-Pt than those treated with CRGD-NG carrying solely cisplatin.
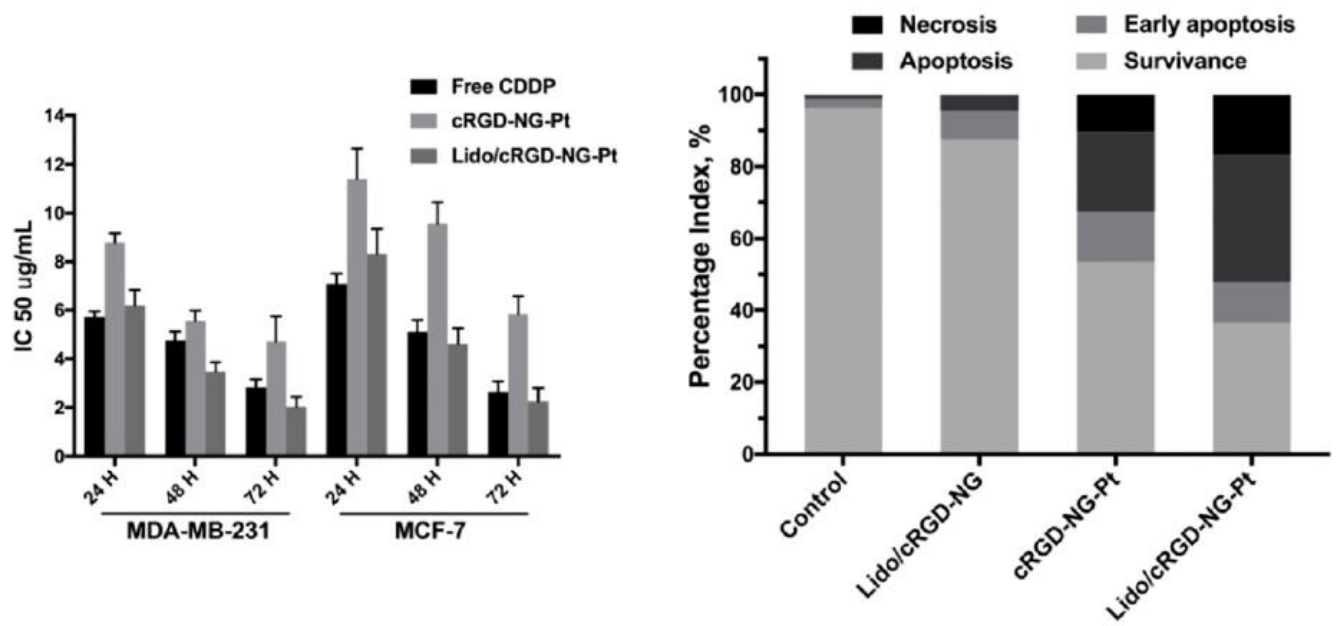

Figure 3. cRGD-NG exhibited higher cytotoxicity towards MDA-MB-231 cells than MCF-7 cells due to active targeting with CRGD peptide. The combination of cisplatin and lidocaine increased cytotoxicity as hypothesized. ${ }^{83}$ Reprinted (adapted) with permission from Ref ${ }^{83}$. Copyright 2018 American Chemical Society.

Next, the authors studied the biodistribution of the bare nanogels to evaluate in vivo CRGD targeting. Nanogels were first tagged with DID fluorescent dye and then injected intravenously in MDA-MD-231 tumor bearing mice. Fluorescent imaging showed a steady 
increase in fluorescence at the tumor site during 24 hours as targeted nanogels accumulated in that region (Figure 4).

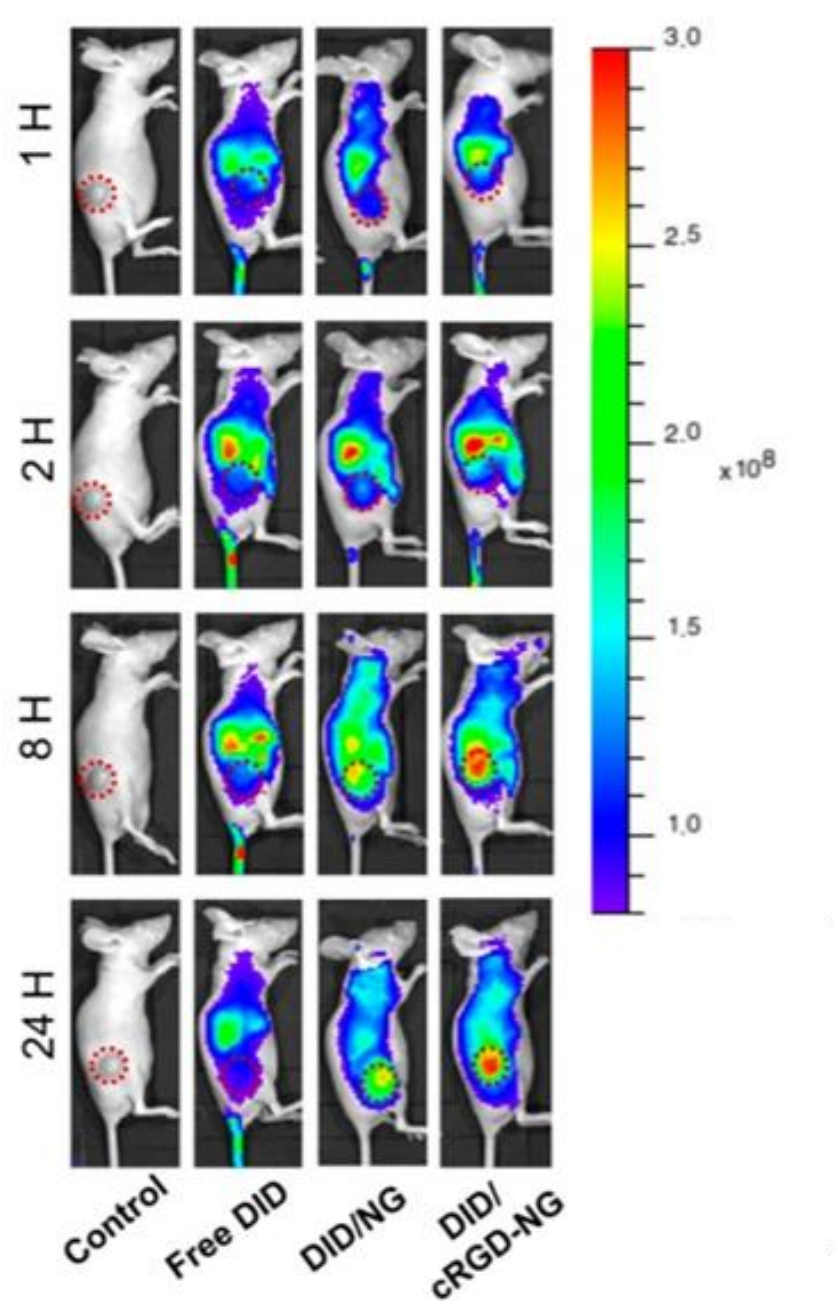

Figure 4. DID-dyed cRGD-NG exhibited increased nanogel trafficking to the MDA-MB-231 tumors owing to the $\alpha v \beta 3$ integrins targeting moiety on the nanogel surface. ${ }^{83}$ Reprinted (adapted) with permission from Ref ${ }^{83}$. Copyright 2018 American Chemical Society.

Furthermore, the authors demonstrated suppression of primary tumor as well as metastasis with negligible weight change in mice treated with Lido/cRGD-NG-Pt. 
$\mathrm{pH}$-responsive nanogels were synthesized by the group of Ravi to topically treat the posterior segment of the eye. ${ }^{52}$ Treatments involving direct injection into the eye have experienced significant improvements over the last few years, however, better patient compliance is still achieved through topical drug delivery. As such targeted nanogels were synthesized to reach retinal pigmented epithelium (RPE) cells. A conjugate of hyaluronic acid (HA), cystamine and cholesterol was synthesized that forms nanogels once dispersed in deionized water. HA is a naturally occurring negatively charged polysaccharide that serves as targeting ligand for CD44 membrane receptors typically found on RPE and Muller cells. ${ }^{87-88}$ Coating nanogels with HA has the added benefit of decreasing their susceptibility to reactive oxygen species and enhancing their cellular uptake by ARPE-19 cells. ${ }^{89}$ Cystamine imparts biodegradability and redox-responsiveness to the nanogel due to its acid-labile disulfide bond. ${ }^{90}$ The addition of cholesterol moieties increased the hydrophobicity of the nanogel facilitating the encapsulation of hydrophobic drugs. $6,38,72$, 91-92 The nanogels were covalently functionalized with carbon dots (luminescent carbon nanoparticles) to track their mobility in cells and with penetratin peptide to enhance intraocular absorption. ${ }^{93}$ The carbon dots - containing surface amine groups, were conjugated to HA on the nanogels through NHS/EDC coupling. Uptake studies with ARPE19 cells indicated that nanogels functionalized with penetratin (HA-cys-CH/P) were taken up more than nanogels absent of penetratin peptides (HA-cys-CH). To further demonstrate the chromophore delivering ability of $\mathrm{HA}-\mathrm{cys}-\mathrm{CH} / \mathrm{P}$, it was loaded with 9-cisretinal and incubated with RPE65-deficient mouse cells. Indeed, upon exposure to green light transretinal rod amplitude and sensitivity were restored to almost the same level achieved by pure, unbound 9-cis-retinal. Delivery efficiency was also tested in vivo by topical administration. Fluorescein was conjugated to 9-cis-retina-loaded $\mathrm{HA}-\mathrm{cys}-\mathrm{CH}$ and 9-cis-retinal-loaded HA-cys- $\mathrm{CH} / \mathrm{P}$ as opposed to carbon dots. Fluorescein was first aminated followed by EDC/NHS coupling to the nanogels. Only 9-cis-retinal-loaded HAcys- $\mathrm{CH} / \mathrm{P}$ achieved partial rod sensitivity restauration. Neither 9-cis-retinal-loaded HA-cys- 
$\mathrm{CH}$ nor free 9-cis-retinal were able to restore sensitivity; indicating the crucial role of penetratin in passage through ocular barriers.

\subsection{Nanogel surface functionalization with proteins}

\subsubsection{Nanogel surface functionalization with Concanavalin A}

In the ongoing pursuit of effective anti-cancer drugs, the group of Mugo and Serpe synthesized multi-responsive NIPAM nanogels containing Concanavalin A (Con A), transferrin and glycogen immobilized on its surface ${ }^{94}$ Con $A$ is a protein that binds to carbohydrates (lectin) that are responsive to changes in $\mathrm{pH}$. At pH 5, Con A transitions from a tetramer to a dimer. ${ }^{95} \mathrm{Glycogen}$ was conjugated to Con $\mathrm{A}$ by exploiting sugar-lectin biorecognition. ${ }^{96}$ The surface biocoating consisted of 4 repeating sets of Con A-glycogen, endcapped with transferrin to target cancer cells. ${ }^{97-98}$ Glucose is expected to compete with glycogen to bind Con A through hydrogen bonding. The authors thus hypothesized that a higher concentration of glycose would lead to a more porous nanogel coating, enabling cargo release. Nanogels (NG-CGT) were loaded with DOX to study its release at various conditions (Figure 5). DOX release was significantly higher at $\mathrm{pH}=4$ than $\mathrm{pH}=7.4$ as the biocoating is degraded in an acidic environment. As the nanogels contain polyNIPAM functionality, they are expected to exhibit a lower critical solution temperature. When the nanogels were heated above $36^{\circ} \mathrm{C}$, at $\mathrm{pH} 7.4$, DOX release remained the same compared to $25^{\circ} \mathrm{C}$. However at $\mathrm{pH} 4$, DOX release increased significantly. It is suspected that solely increasing the temperature does not influence DOX release as the nanogel coating remains intact. 
(a)

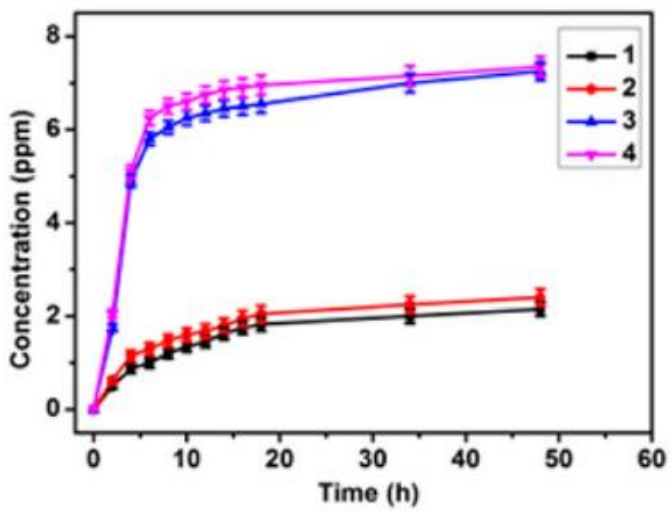

(c)

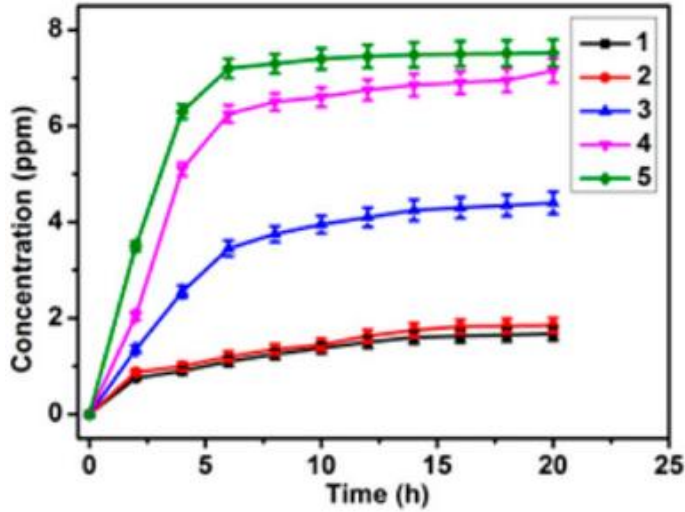

(b)

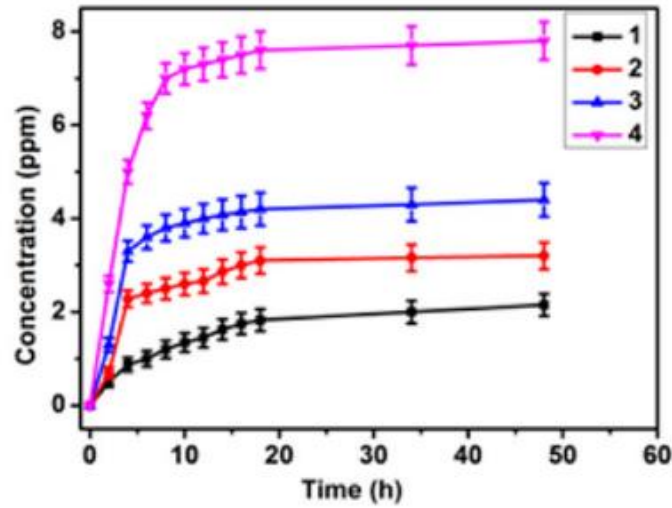

(d)

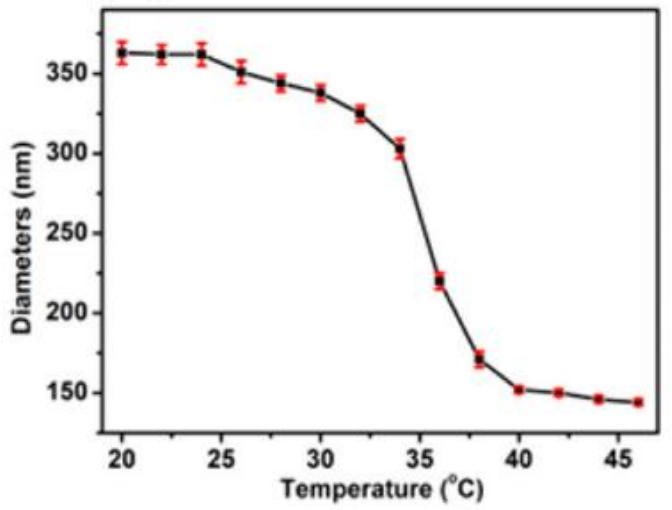

Figure 5. DOX-release was studied at various conditions. Graph a displays the DOX-release profile at (1) $\mathrm{pH}=$ 7.4; (2) $\mathrm{pH}=6$; (3) pH = 5 and (4) pH = 4. Chart $b$ is the release profile of DOX-loaded NG-CGT at pH = 7.4 with varying glucose concentrations such as (1) 400 ppm, (2) 700 ppm, (3) 1000 ppm and (4) 1300 ppm. Graph c displays the behavior of NG-CGT at various combinations of $\mathrm{pH}$ and temperature such as (1) $\mathrm{pH}=7.4+25^{\circ} \mathrm{C}$; (2) $\mathrm{pH}=7.4+37{ }^{\circ} \mathrm{C}$; (3) pH $=4+25^{\circ} \mathrm{C}$; (4) pH $=4+37^{\circ} \mathrm{C}$ and (5) $\mathrm{pH}=4+45^{\circ} \mathrm{C}$. Lastly, graph d displays the size of the nanogel as a function of temperature. ${ }^{94}$ Reprinted (adapted) with permission from Ref ${ }^{94}$. Copyright 2017 American Chemical Society.

Nanogels endcapped with transferrin (NG-CGT) were compared to those lacking this end group (NG-CG). DOX-loaded NG-CGT exhibited higher fluorescence, and thus higher 
cellular uptake in HepG2 liver cancer cells than DOX-loaded NG-CG, confirming active targeting.

\subsubsection{Nanogel surface functionalization with albumins}

In an effort to synthesize immunotherapeutic vaccines for cancer, Li et al. synthesized cationic dextran nanogels by means of inverse mini-emulsion photo-polymerization. ${ }^{99} \mathrm{Egg}$ albumin (OVA), a protein antigen, was reacted with succinimidyl S-acetylthioacetate (SATA) through its primary amines. It was then loaded into the nanogel followed by thiol deprotection with hydroxyl amine. Lastly conjugation via thiol-disulfide exchange afforded SATA-OVA nanogels with labile disulfide bonds for OVA release at a certain $\mathrm{pH}$ (Scheme 6). As comparison, native OVA was physically entrapped in the nanogel matrix.
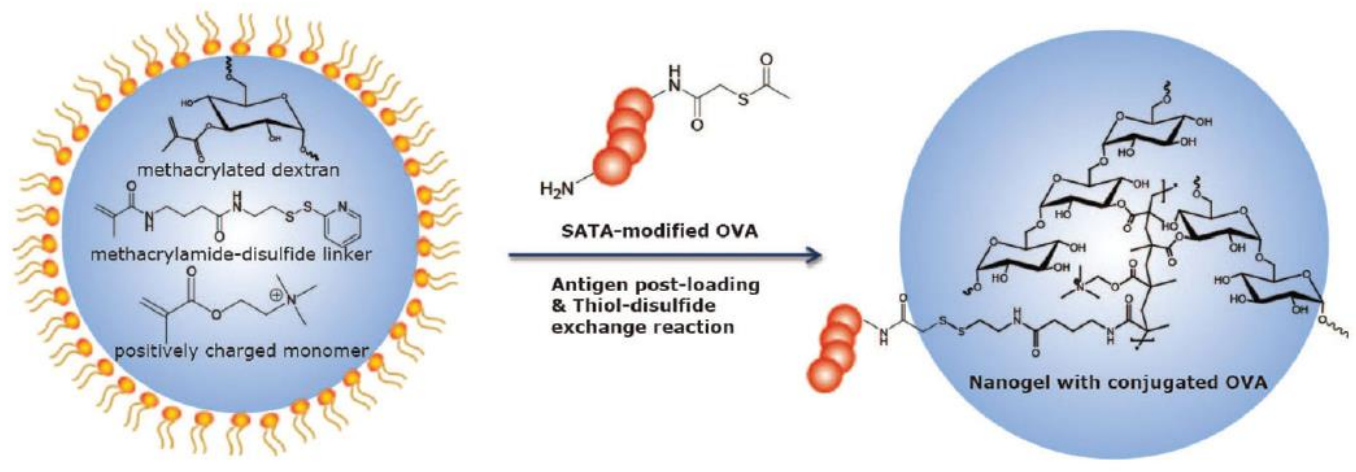

Scheme 6. Li et al synthesized cationic dextran nanogels with a disulfide functionality. The nanogels were loaded with native OVA or covalently bound to SATA-functionalized OVA. ${ }^{99}$ Reproduced and adapted with permission from The Royal Society of Chemistry.

The influence of size and surface charge of nanogels loaded with native OVA was studied on dendritic cells as they play a vital role in the body's immune response. ${ }^{100-101}$ Positively 
charged nanogels were efficiently internalized in the cells as opposed to neutral nanogels and microgels, as expected. ${ }^{102-105}$ Cationic nanoparticles are able to influence the maturing and specialization of dendritic cells. ${ }^{106}$ D1 cells in particular will express CD40, CD86 and IL-12 when mature. It was noted that the higher the surface charge, the more CD40, CD86 and IL-12 were upregulated. Cells incubated with neutral nanogels did not exhibit upregulation; CD40, CD86 and IL-12 expression was similar to that of mature unstimulated CD1 cells. Nanogels containing SATA-OVA covalently bound to the matrix were shown to be endocytosed starting from $2 \mathrm{~h}$ of incubation with D1 cells. After 24 hours the nanogels were still located in the lysosomes. In a next step OVA was heavily labeled with BODIPY (DQ-OVA). BODIPY has the unique capability of quenching its own fluorescence when various of its molecules are in close proximity to each other. When DQ-OVA is loaded or conjugated to the nanogel its green fluorescence is quenched. However, if the loaded/conjugated DQ-OVA is released from the nanogel it will once again fluoresce. Cell experiments with D1 cells indicated that after $5 \mathrm{~h}$ green fluorescence started emerging and becoming brighter after $24 \mathrm{~h}$. The green fluorescence was homogenously distributed throughout the cytosol indicating that the nanogel had released DQ-OVA. Interestingly, uptake of the nanogels did not differ for conjugated OVA compared to native loaded OVA. However, DQ-OVA fluorescence was five times higher for conjugated OVA than native loaded OVA, demonstrating the importance and usefulness of nanogel surface modification. Mice inoculated with B16-OVA melanoma (that expresses OVA antigens), were immunized with free OVA or OVA-loaded nanogels. The tumor growth and life expectancy was the same for both groups as OVA is held in the loaded nanogels through electrostatic interactions, meaning quick release. Nanogels covalently bound to OVA through labile disulfide bonds had the lowest mortality rate and smallest tumor size compared to all other formulations; except when these nanogels were supplemented with adjuvant polyinosinic-polycytidylic acid. Conjugated-OVA nanogels devoid of any adjuvants expressed the same amount of $\mathrm{CD} 8^{+} \mathrm{T}$ cells as supplemented 
OVA-loaded nanogels and supplemented free OVA. Supplemented conjugated-OVA nanogels had the highest immune response, producing the most $\mathrm{CD} 8^{+} \mathrm{T}$ cells. This formulation had quite interesting results on tumor growth. Two out of the 10 mice treated exhibited rapid tumor growth, four displayed tumor growth suppression and the remaining four mice experienced complete tumor elimination. This demonstrates the great potential of this work as a possible cancer vaccine.

\subsection{Nanogel surface functionalization with antibodies}

Adamo et al. synthesized amino-functionalized nanogels by e-beam irradiation, followed by the conjugation of monoclonal antibodies (mAbs) antiCD44 and anti- $\beta 3$ integrin on the surface to achieve active targeting. ${ }^{107}$ After confirming faster cell uptake for targeted nanogels, cell studies were conducted to determine the uptake pathway of antiCD44nanogel. ECV304 cells were first treated with various inhibitors such as phenylarsine oxide, nystatin and 5-(N-ethyl-N-isopropyl)amiloride (EIPA). Each inhibitor impedes a certain uptake pathway. Analyzing the uptake efficiency for each inhibitor and determining the one with the lowest amount of endocytosed nanogels, reveals the primary pathway by which the nanogels are taken up in ECV304 cells. The uptake experiment was also conducted at $4^{\circ} \mathrm{C}$ as only passive uptake occurs at this temperature. The lowest cell uptake is achieved when macropinocytosis is inhibited with EIPA and when the experiment is conducted at $4^{\circ} \mathrm{C}$. Interestingly, treatment with EIPA also affected the morphology of the ECV304 cells. Anti- $\beta 3$ integrin nanogels were subsequently tested for their targeting of activated endothelial cells. During a so-called "scratch-wound assay" confluent cells are scratched resulting in a wound. Many processes occur in nearby cells to heal the wound such as migration to the location of the scratch and expression of 
certain integrins on the active endothelial cell membranes. Colocalization studies confirmed active targeting of anti- $\beta 3$ integrin nanogels to these active endothelial cells.

In the field of adoptive transfer researchers aim to increase the body's immune response by harvesting the patient's T cells (a type of white blood cells), enhancing its immune properties and transferring it back in the patient's body. Activated T cells exhibit a higher reducing rate than non-activated ones, as more free thiols are present on the surface of activated T cells. ${ }^{108}$ Upon binding with antibodies (such as anti-CD45), the surface reducing rate further increases. Exploiting this mechanism, Tang et al. synthesized protein nanogels that are responsive to T cell receptors (TCR). ${ }^{109}$ Once bound, the disulfide bonds of the antibody-functionalized nanogel - originating from the crosslinker, are reduced. As a result, the nanogel collapses into its cytokine building blocks (Scheme 7).

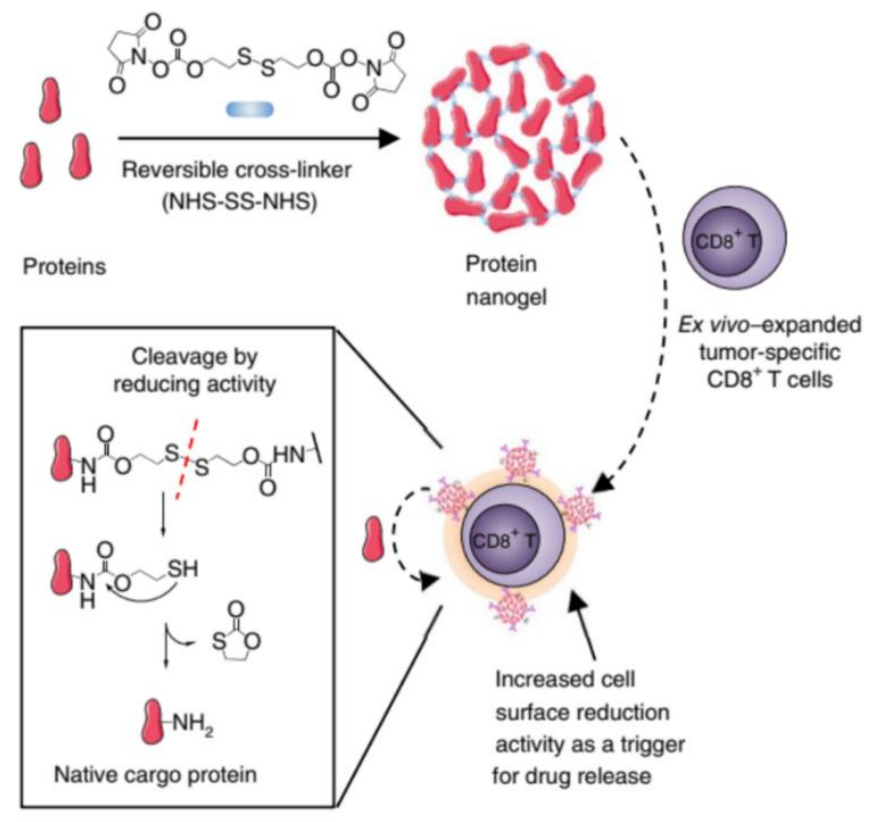

Scheme 7. Cytokine proteins are crosslinked to a nanogel with a disulfide moiety. Upon binding to an activated T cell disulfide bonds are reduced resulting in its collapse and release of its native cargo. ${ }^{109}$ Reproduced and adapted with permission from Springer Nature. 
In this way higher dosing is achieved - up to $8 \mu \mathrm{g} \mathrm{IL-15Sa} \mathrm{per} \mathrm{million} \mathrm{T} \mathrm{cells,} \mathrm{as} \mathrm{the} \mathrm{nanogel}$ is approximately $92 \%$ protein. The promising cytokine interleukin-15 super-agonist complex (IL-15Sa) $)^{110-112}$ was crosslinked to a nanogel with a reversible and degradable disulfide crosslinker; followed by surface modification with antibody CD45 (anti-CD45) to achieve active targeting. Anti-CD45 hinders internalization of the cytokine-NG by the T cells. The nanogels were further surface functionalized with $P E G_{5 k}-P L L_{33 k}$ to provide a cationic coating and enhance T cell coupling. Non-degradable nanogels were synthesized as described above with the exception of using a nondegradable crosslinker. T cells proliferate during an immune response by cloning themselves, producing enough cells for a proper defense. This process is called T cell expansion. Anti-CD45/IL-15Sa NGs produced the highest expansion in T cells compared to the same amount of free IL-15Sa and nondegradable anti-CD45/IL-15Sa NGs. Similar results were also found for in vivo experiments. Anti-CD45/IL-15Sa NGs were able to enhance expansion starting at a dose of $30 \mathrm{ng}$ IL-15Sa per million T cells. These nanogels were also highly selective; only expanding the adoptive transferred $\mathrm{CD}^{+} \mathrm{T}$ cells and not endogenous $\mathrm{T}$ cells. Expansion was increasingly higher in the presence of antigens. Lastly, IL-15Sa administered freely exhibited immunotoxicity, resulting in the eventual death of mice that received $>10 \mu \mathrm{g}$. Contrarily, anti-CD45/IL-15Sa NGs did not exhibit toxicity, even at its maximum dose of 80 $\mu \mathrm{g}$ IL-15Sa per $10^{7} \mathrm{~T}$ cells.

\subsection{Nanogel surface functionalization with (co)enzymes}

Gama and coworkers synthesized biotin-labeled mannan and dextrin nanogels through a 1,4-addition reaction to improve the adjuvant response of vaccines. ${ }^{113}$ Biotin is a vitamin as well as a co-enzyme. Biotinylation is also often used to target streptavidin or avidin. The nanogels were then loaded with ovalbumin (OVA) to function as a model antigen. An 
efficient vaccine will transport the antigen to antigen presenting cells (APCs) such as macrophages and dendritic cells. ${ }^{114}$ These cells will initiate an immune response. When vaccines are administered intramuscularly or subcutaneously they migrate to the lymph nodes and initiate a secondary immune response. The authors tested the vaccine efficiency of the nanogels by injecting them into the paw pads of rats and collecting their lymph nodes after $24 \mathrm{~h}$. Both mannan and dextrin nanogels were found in the lymph nodes. BALC/c mice treated with OVA-loaded nanogels exhibited higher levels of IgG1 anti-OVA when injected with mannan nanogels as opposed to dextrin nanogels and OVAPBS dispersion.

Collagenase is an enzyme capable of cleaving peptide bonds of collagen. The group of Tang immobilized collagenase on alginic acid-OEAM nanogels (ALG NGs) through EDC/NHS coupling forming pH-sensitive nanogels (Co@ALG NGs) with enhanced extracellular matrix penetrating abilities (Scheme 8). ${ }^{115}$ OEAM is a dimethacrylamide crosslinker formerly developed by the group; which contains an acid-labile ortho ester bond. ${ }^{116} \mathrm{pH}$-triggered DOX release indeed exhibited higher release at lower $\mathrm{pH}$ for both Co@ALG NG and ALG NG when loaded with DOX.

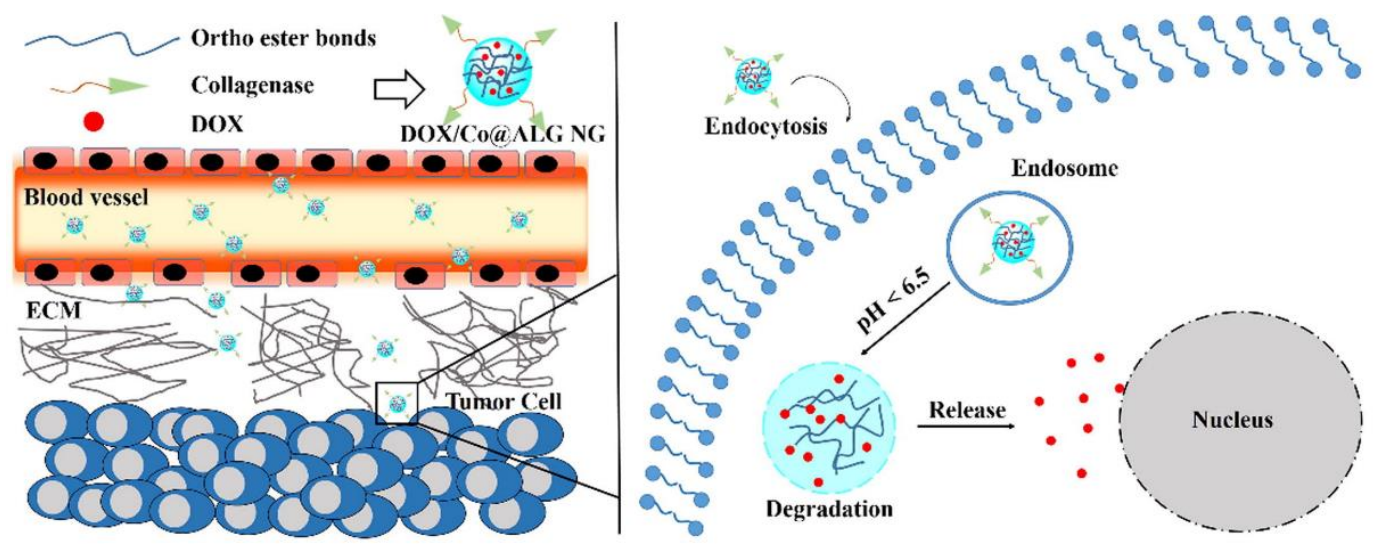

Scheme 8. Collagenase coating enhances the penetrating abilities of nanogels, increasing drug delivery through the extracellular matrix (ECM). ${ }^{115}$ Reproduced with permission from Elsevier. 
To demonstrate the retained enzyme activity of collagenase once bound to ALG NGs, various formulations of nanogels were incubated with gelatin hydrogel (Figure 6). Empty ALG NG, free DOX and DOX/ALG NG did not degrade the gelatin hydrogel even after 24 hours. Free collagenase, Co@ALG NG and DOX/Co@ALG however were both able to liquefy gelatin by cleaving its peptide bonds. After 24 h of incubation with Co@ALG NG and DOX/Co@ALG the gelatin remained in a liquid state.
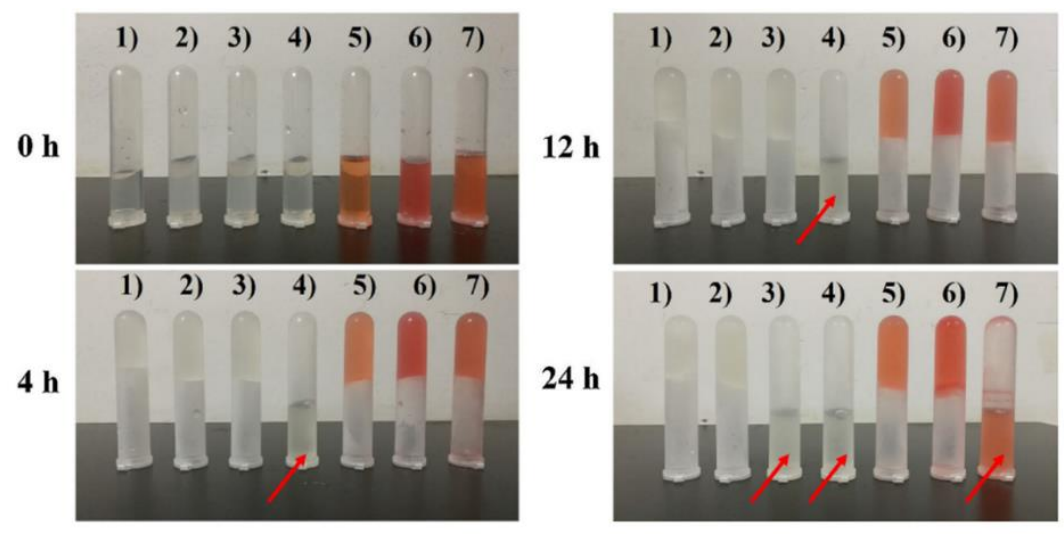

Figure 6. Various formulations of nanogels were incubated with gelatin hydrogel to assess the enzyme activity of (bound) collagenase during 24 hours. Tube 1 contains pure gelatin; tube 2 ALG NG; tube 3 Co@ALG NG; tube 4 collagenase; tube 5 free DOX; tube 6 DOX/ALG NG and tube 7 DOX/Co@ALG NG. If enzyme activity is retained, collagenase will break down gelatin resulting in a liquid. ${ }^{115}$ Reproduced and adapted with permission from Elsevier.

Cellular uptake studies were conducted in HepG2 and H22 cancer cells and the accumulation and distribution of DOX was observed. For DOX/ALG-NG and DOX/Co@ALG, red fluorescence originating from DOX was observed in the cytoplasm and cell nuclei, indicating successful nanogel uptake, DOX release and DOX trafficking to the cell nucleus. Red fluorescence was significantly lower when cells were treated with free DOX. Tang and coworkers proceeded by quantifying the cytotoxicity of the DOX-loaded nanogels. For HepG2 cells, cell viability decreased to $48 \%$ when treated with DOX/ALG-NG, to $45 \%$ for 
free DOX and to 35\% when treated with DOX/Co@ALG at $16 \mu \mathrm{g} / \mathrm{mL}$. In the case of $\mathrm{H} 22$ cells, DOX/ALG-NG exhibited a cell viability of 47\% (similar to free DOX) and DOX/Co@ALG exhibited a cell viability of $38 \%$. Nanogels were coated with collagenase to achieve enhanced extracellular matrix penetration. The results mentioned above seem to support this hypothesis. However, cell monolayers are not proper representations of the extracellular matrix of solid tumors. Therefore, uptake and cell viability studies were repeated on three-dimensional HepG2 multicellular spheroids. Indeed, as hypothesized, functionalization with collagenase endowed the nanogels with increased penetration abilities, resulting in higher cytotoxicity. In vivo studies further solidified this conclusion as tumors from mice treated with DOX/Co@ALG NGs grew the slowest and had the lowest tumor volume and weight at the end of the study. The tumor growth inhibition was calculated for free DOX, DOX/ALG NG and DOX/Co@ALG NG. The values were 48.3\%, $70.3 \%$, and $77.6 \%$, respectively.

\subsection{Nanogel surface functionalization with (poly)sugars}

\subsubsection{Nanogel surface functionalization with sugars}

Research into creative ways to achieve successful cellular uptake of nanogels while bypassing the inherent cytotoxicity associated with the cationic charges of gene vectors was conducted. Akiyoshi et al. synthesized enzyme-responsive nanogels with an enzymatically transformable surface to reduce the cytotoxicity associated with cationic charges. ${ }^{117}$ Poly (L-lysine) was modified with cholesterol units and underwent reductive amination with maltopentaose (a sugar pentamer). Self-assembly of the polypeptide in PBS buffer resulted in a physically crosslinked nanogel. The nanogels were then subjected to tandem enzymatic polymerization with glycogen phosphorylase and glycogen 
branching enzyme to covalently coat a layer of branched polysaccharide over the nanogels (Scheme 9).
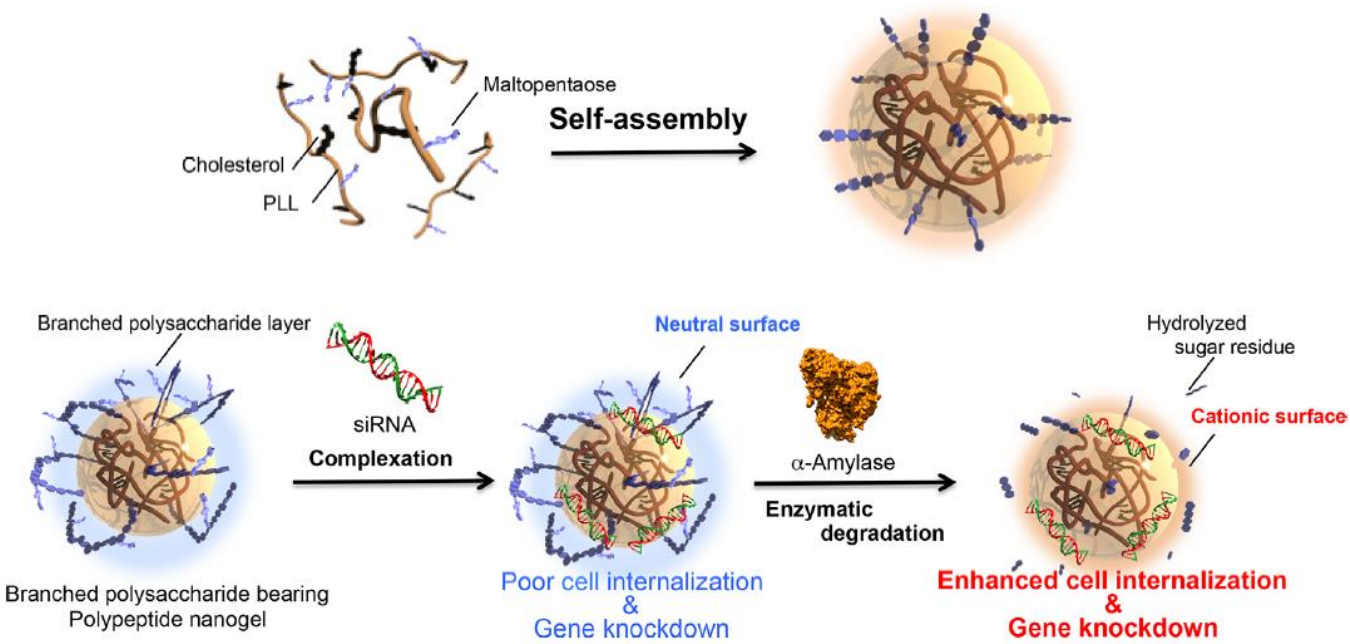

Scheme 9. Nanogels were formed through self-assembly of cholesterol-modified poly(L-lysine) and maltopentose. Subsequently a layer of polysaccharide was bound to the surface by enzymes glycogen phosphorylase and glycogen branching enzyme. The resulting sugar-decorated nanogel can mask the negative charge of siRNA until its coating is hydrolyzed by $\alpha$-amylase. ${ }^{117}$ Reprinted (adapted) with permission from Ref 117. Copyright 2017 American Chemical Society.

This layer served as a neutral barrier between the cationic charges on the surface of the nanogel and other charged components present in blood to avoid both non-specific complexation/aggregation and repulsion. As a result, the coated nanogels exhibited low cytotoxicity up to $100 \mu \mathrm{g} / \mathrm{mL}$ in Renca cells. Interestingly, the neutral, coated nanogels were still able to efficiently bind siRNA starting from nanogel/siRNA ratios greater than 2 . The polysaccharide coating was successfully hydrolyzed by $\alpha$-amylase at serum concentrations, exposing the poly(L-lysine) core and thus increasing the nanogel surface charge. The positive $\zeta$-potential enhanced cellular uptake and gene silencing effects in Renca cells incubated with both siRNA/nanogel complexes and $\alpha$-amylase, resulting in a 
$50 \%$ decrease in gene expression. Those incubated with solely the complexes exhibited a $20 \%$ decrease.

\subsubsection{Nanogel surface functionalization with DNA}

Nanogels have been lauded for their properties and versatility; both in literature and in this review. A great example of their range is described by Thelu et al. They synthesized nanogels comprising of solely DNA, protein and biotin. ${ }^{118} \mathrm{~A}$ four-armed branched DNA macromolecule was synthesized containing biotin units at the end of each arm. Through self-assembly and multivalent interactions with streptavidin, a nanogel was formed. When aptamer DNA was included in the self-assembly, these moieties were attached to the nanogel surface, targeting it towards HeLa and CCRF-CEM cells (Figure 7). To probe cellular uptake and selectivity, the nanogels were dyed with Cyanine and incubated with HeLa, CCRF-CEM and Ramos cells. Ramos cells do not express the receptor for this aptamer. Fluorescence assisted cell sorting (FACS) and confocal laser scanning microscopy (CLSM) measurements confirmed only a minimum amount of uptake for Ramos cells compared to HeLa and CCRF-CEM cells. Cell viability studies conducted with DOX indicated that its toxicity towards cancer cells is not negatively affected when encapsulated in the nanogel but remains the same. 
a)

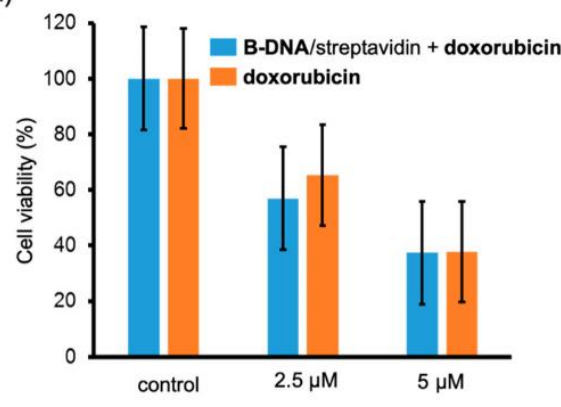

b)

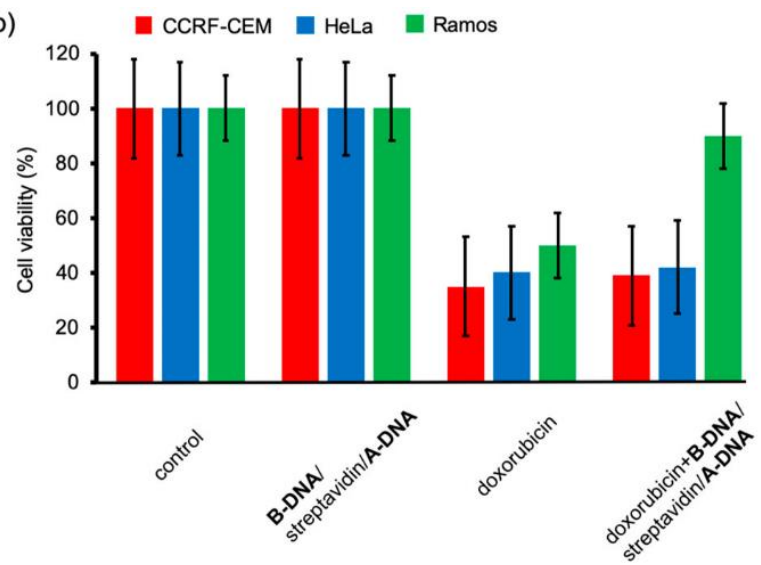

Figure 7. Thelu et al. synthesized nanogels from DNA, streptavidin and biotin. Surface conjugation with aptamer DNA targeted the nanogels towards HeLa cells and CCRF-CEM cells. The nanogels were loaded with toxic DOX and incubated with various cells. DOX remained potent even when encapsulated (a). The modified nanogels exhibited higher cytotoxicity towards HeLa and CCRF-CEM cells indicating active targeting. ${ }^{118}$ Reprinted (adapted) with permission from Ref ${ }^{118}$. Copyright 2019 American Chemical Society.

\subsection{Nanogel surface functionalization with drugs}

In drug delivery, therapeutics are mostly encapsulated in nanogel carriers or bound by weak electrostatic bonds ${ }^{119-121}$; often resulting in drug leakage. By covalently binding the drugs to the nanogel a so-called prodrug nanogel is formed; increasing the stability and often also the efficacy of the drug. ${ }^{122-130}$ Peng Liu and co-workers synthesized nanogels consisting of PEGylated oxidized alginate (a pH-sensitive natural polysaccharide), $\alpha$ cyclodextrin and cystamine (Scheme 10). ${ }^{131}$ These nanogels were subsequently surface decorated with DOX via an acid-labile Schiff base linkage, affording dual-responsive prodrug nanogels. Cystamine served as a biodegradable crosslinker as it contains a labile disulfide bond. Prodrug nanogels were also obtained by switching the order of the synthesis steps and by incorporating $\beta$-cyclodextrin as opposed to $\alpha$-cyclodextrin. 


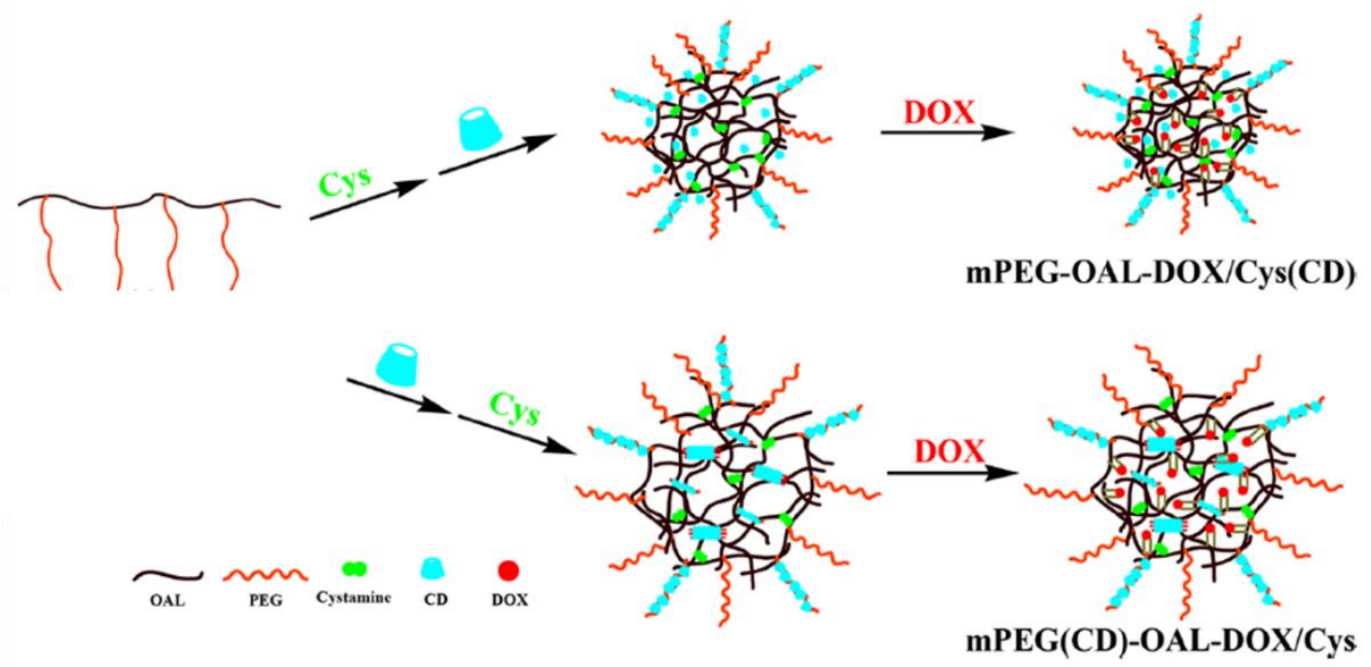

Scheme 10. Nanogels were synthesized comprising of $\alpha / \beta$ cyclodextrin and cystamine. Their surface was subsequently conjugated with DOX, producing dual-responsive nanogels. Depending on whether cyclodextrin or cystamine was incorporated first influenced the size of the final nanogel. ${ }^{131}$ Reprinted (adapted) with permission from Ref ${ }^{131}$. Copyright 2018 American Chemical Society.

Transmission electron microscopy (TEM) images show that crosslinking after complexation of $\alpha$-cyclodextrin resulted in more uniform nanogels and aggregated less than with $\beta$-cyclodextrin. Nanogels in which cyclodextrin was incorporated prior to crosslinking with cystamine had much higher DOX release in tumor simulating media regardless of which cyclodextrin was incorporated. The reason for the differences between the different prodrug nanogels is due to the formation of polyrotaxanes by the cyclodextrin units. ${ }^{132}$ Both $\alpha$ - and $\beta$-cyclodextrin can non-covalently bind with multiple other cyclodextrin molecules forming a supramolecular thread that weaves over the PEG chains forming so-called polyrotaxanes. However, $\alpha$-cyclodextrin has a smaller inner cavity than $\beta$-cyclodextrin resulting in variations in the structures of their corresponding nanogels. In addition, when cyclodextrin is added to the nanocomplex prior to crosslinking, polyrotaxanes are formed uniformly over the whole nanogel matrix. If 
however crosslinking is followed by addition of cyclodextrin, polyrotaxanes are only formed around the available PEG chains that are not crosslinked in the nanogel core. These differences in the prodrug nanogel constructs affect the diffusion and thus the release profiles of the conjugated DOX. Cell viability studies with HepG2 cells demonstrated similar cytotoxic activity of free DOX on cancer cells as DOX grafted onto the surface of the prodrug nanogel.

Wang and Dai et al. also synthesized DOX prodrug dual-responsive nanogels. Their one-step self-assembly assisted methodology consisted of crosslinking dextran and (meth)acrylic acid with a disulfide-containing crosslinker. The redox responsive nanogel was then conjugated with DOX by first functionalizing with hydrazinium hydroxide, followed by the addition of DOX via an acid-labile hydrazone bond (Dex-SS-PAA-DOX NG). ${ }^{133}$ The resulting biodegradable polysaccharide nanogel responds to both redox and acidic stimuli, making it an ideal drug carrier for the weakly acidic ${ }^{134-135}$ and reductive ${ }^{136-}$ 137 microenvironment of cancer cells. The inclusion of dextran in the nanogel matrix enhances cellular uptake by stimulating endocytosis. ${ }^{6}$ Initial studies confirmed the $\mathrm{pH}$ responsiveness of Dex-SS-PAA-DOX NG as a lower $\mathrm{pH}$ resulted in higher DOX release. In vitro experiments indicated that Dex-SS-PAA-DOX is toxic to cancerous cells such as MCF7 and MDA-MB-231 breast cancer cells, whereas healthy human umbilical vein endothelial (HUVEC) cells remained unaffected. At $5 \mu \mathrm{g} / \mathrm{mL}$ of DOX in Dex-SS-PAA-DOX, MDA-MB-231 cells exhibited a cell viability of $18 \%$. Free DOX was internalized at a much higher rate than Dex-SS-PAA-DOX NG, suggesting that the nanogels are taken up by endocytosis and not diffusion. After $12 \mathrm{~h}$, cells incubated with free DOX exhibited high fluorescence in the cell nuclei. Cells incubated with Dex-SS-PAA-DOX, however, had higher fluorescence in the cytoplasm as cellular uptake was still active and DOX release was not yet prevalent. 

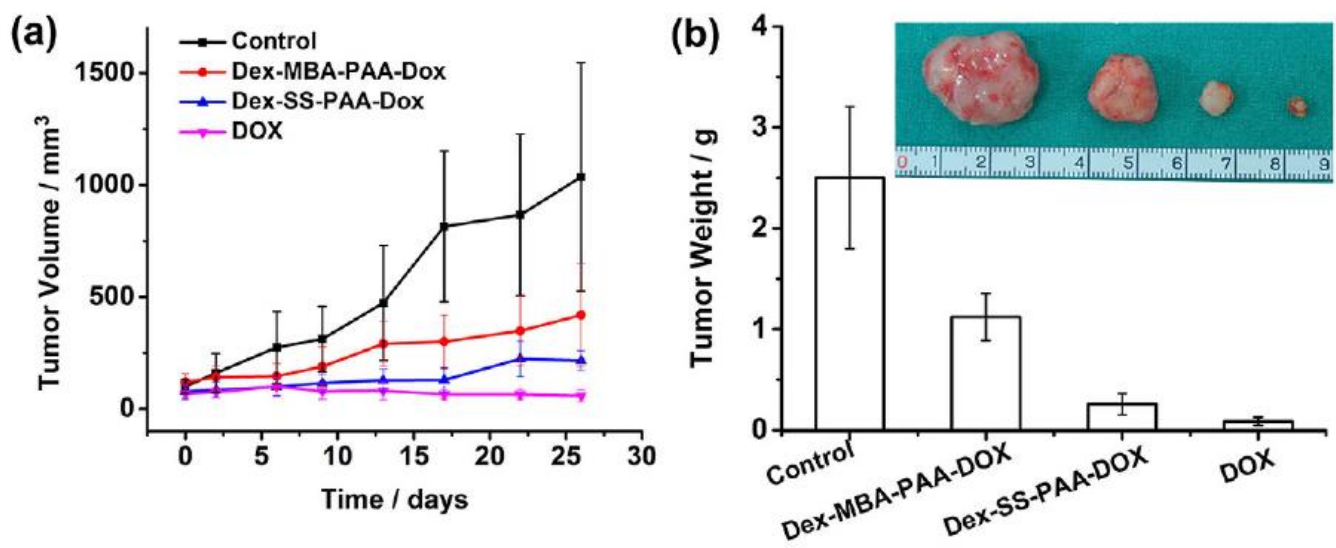

Figure 8. Mice wear treated with free DOX, Dex-SS-PAA-DOX NGs and Dex-MBA-PAA-DOX NGs that do not contain a disulfide functionality. Mice treated with Dex-SS-PAA-DOX NGs exhibited the slowest tumor growth barring free DOX. ${ }^{133}$

Following the success of the in vitro studies the authors assessed the antitumor effect of the nanogels in nude MDA-MB23 tumor bearing nude mice. Mice treated with Dex-SSPAA-DOX NGs displayed an $80 \%$ decrease in tumor volume compared to the control (Figure 8). Equivalent nanogels synthesized with a crosslinker that does not contain a disulfide (Dex-MBA-PAA-DOX NGs) exhibited a 60\% decrease. This is due to these nanogels being mono-responsive and thus only releasing DOX as a response to $\mathrm{pH}$. Free DOX exhibited the highest tumor inhibition. However, as it is free and not targeted, DOX does not discriminate between healthy and cancerous cells. As a result, mice treated with free DOX exhibited rapid weight loss and organ damage.

\subsection{Functional nanogels for in vitro studies}

Nanogels are often utilized as carriers in biomedical applications as they have ideal properties for binding, transporting and releasing therapeutics at target site. Nanogels 
can also enhance circulation time, increase efficacy and targeting of its load. As mentioned previously, nanogels have found use in cancer research, function as antifouling agent and are able to overcome multi-drug resistance. Versatile nanogels are applied in ophthalmic treatments, wound healing, immunotherapy, vaccines and gene delivery. This section will focus primarily on cellular uptake of nanogels in various fields of research as well as the mechanisms by which they are internalized in cells.

\subsection{Nanogels for drug delivery}

\subsubsection{Nanogels in cancer treatment}

Wu and He employed a unique strategy to ensure sufficient uptake of cancer drugs. As opposed to targeting with ligands, the authors coated their nanocarrier with cell membranes. By coating gelatin nanogels with mesenchymal stem cells automatic affinity towards the surface of cancer cells is achieved. ${ }^{138-140}$ These nanogels (SCMGs) had a coreshell structure which it maintained when internalized by cells. SCMGs experienced higher accumulation in the tumors and lower clearance by the reticuloendothelial system as seen in Figure 9. 

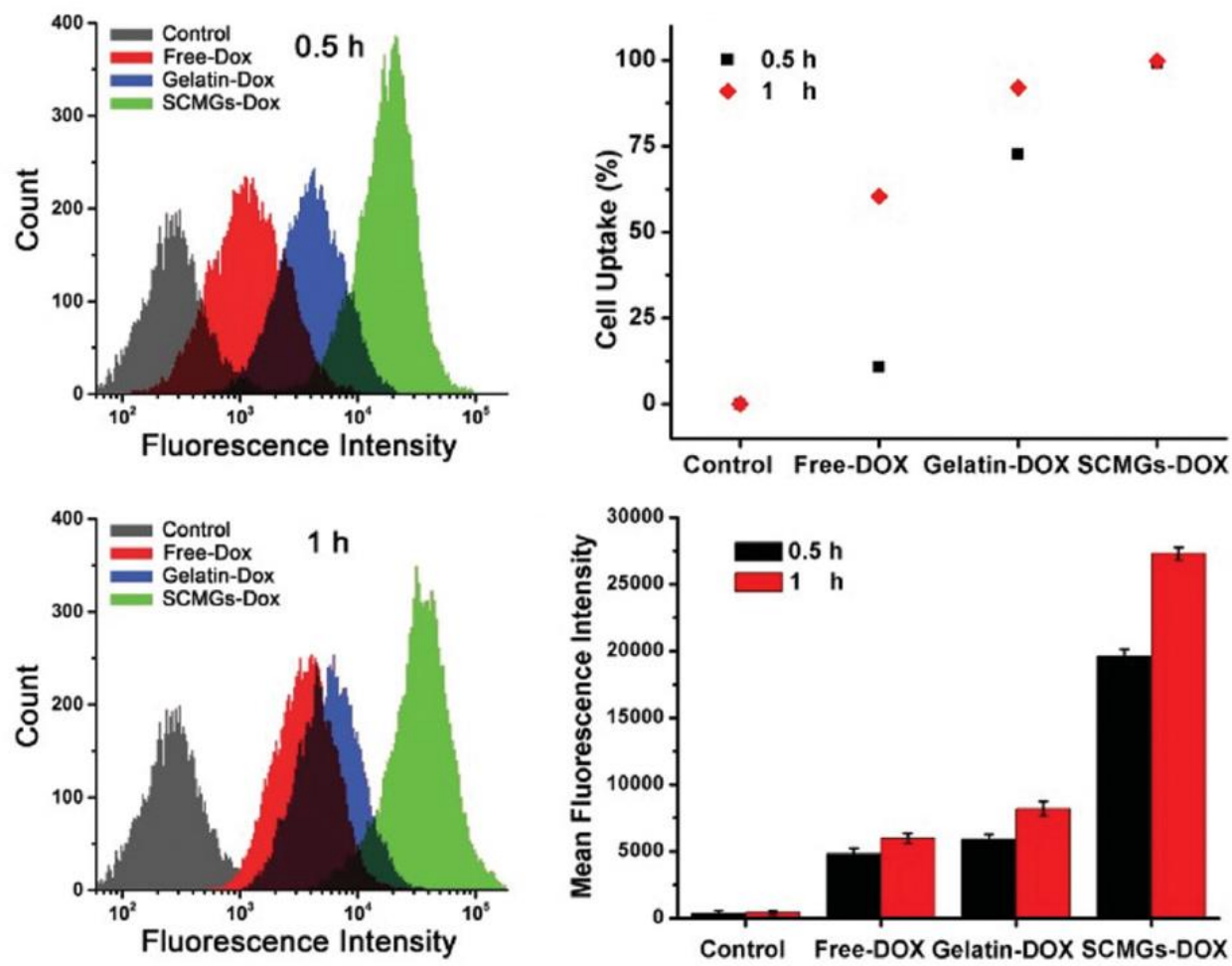

Figure 9. FACS calculations demonstrate higher uptake for SCMGs-DOX compared to free DOX and gelatinDOX. ${ }^{138}$ Adapted and reproduced with permission from John Wiley and Sons.

Mice were treated with PBS, gelatin nanogels, free DOX, gelatin nanogels loaded with DOX and SCMGs loaded with DOX. After 15 days the tumors were excised and weighed. Tumors treated with SCMGs-DOX exhibited the lowest weight gain, whereas those treated with PBS and gelatin increased the fastest in weight and volume.

Therapeutic delivery by means of a carrier has the added benefit of simultaneous delivery of multiple loads to the same site. Co-loading nanogels with anti-tumor drugs as well as dyes enables synergistic treatment. Zhang and coworkers utilized a temperature- 
sensitive NIPAM nanogel to this end. ${ }^{141}$ Site specific delivery is especially desired in chemotherapy as the adverse effects are well known. Employing $\mathrm{pH}$-sensitive nanogels that release their cargo in the acidic microenvironment of tumors is well documented. The team of Zhang opted for signaling of cargo release by an exogenous stimulus, namely light. The nanogel was co-loaded with DOX and near infrared (NIR) dye indocyanine green (ICG). When ICG is radiated with NIR light it releases heat, inducing DOX release from the NIPAM nanogel in the vicinity of cancer cells. This technique is called photothermal penetration and is considered highly specific and minimally invasive. ${ }^{142-144}$ An attractive side-effect of the heat generated is possible thermal ablation of the cancer cells. PolyNIPAM has a volume phase transition temperature (VPTT) of $32^{\circ} \mathrm{C}$. Altering its structure by incorporating hydrophilic monomers such as acrylamide increases the VPTT. To diminish premature drug leakage, nanogels were coated with PEGylated liposomes by performing the polymerization in liposome templates. ${ }^{145-147}$ The cellular uptake of the ICG and DOX-loaded nanogels with a liposome coating (DI-NG@lipo) was studied in 4T1 breast cancer cells. When DI-NG@lipo was incubated with the cells without any external stimulus, DOX release was very low. However when DI-NG@lipo was irradiated with a laser at $\lambda=808 \mathrm{~nm}$ for $5 \mathrm{~min}$, a large DOX release was observed. Colocalization of the DOX and DAPI fluorescence indicated DOX distribution to the cell nucleus. To verify if the enhanced DOX release was photothermally-induced, DI-NG@lipo was heated at $42^{\circ} \mathrm{C}$ for 30 min and its DOX release was observed. Similar results were obtained as for DI-NG@lipo + laser irradiation. Cytotoxicity studies with NG@lipo (no cargo) and I-NG@lipo (only ICG) demonstrated high cell viability up to $1 \mathrm{mg} / \mathrm{mL}$. When the nanogel contained both ICG and DOX, cell viability in the absence of laser irradiation decreased to similar levels as obtained for free DOX. I-NG@lipo in the absence of laser irradiation exhibited a much higher cell viability than when I-NG@lipo is treated with NIR light - demonstrating heat toxicity. The highest cytotoxicity was achieved by DI-NG@lipo in the presence of NIR laser, confirming synergistic cancer treatment. 
Another great advantage of nanogels as delivery vehicles is their ability to be dual targeting. Regular targeting strategies employ a singular mechanism - either recognition of overexpressed receptors or changes in the microenvironment. Combining the two strategies on the same nanogel by employing co-recognition of receptor and environment overcomes their individual limitations. Chen, Ding et al. functionalized polypeptide nanogels with phenylboronic acid (PBA) to target metastatic cancer cells that overexpress sialyl (SA) epitopes on their cell membrane. ${ }^{148-149}$ The nanogels were also conjugated with morpholine (MP) to serve as a charge reversal group and enhance cellular uptake. ${ }^{150-151}$ $\mathrm{MP}$ is quite a convenient targeting ligand as it is either negatively charged or neutral in healthy tissue but becomes positively charged upon exposure to tumor environment possessing a $\mathrm{pH}$ of $6.5 .{ }^{152}$ The nanogels were crosslinked by disulfide bonds which can be cleaved by glutathione (GSH), and were subsequently loaded with DOX to PMNG/DOX. Cell viability studies with PMNG/DOX and PNG/DOX (lacking MP moieties) demonstrated similar behavior at $\mathrm{pH}=7.4$ as both nanogels are neutral. However, PMNG/DOX displayed 1.17 times higher cytotoxicity than PNG/DOX at $\mathrm{pH}=6.5$ owing to its cationic charge and thus higher uptake at this $\mathrm{pH}$. Competition assays with PBA and sialidase (cleaves SA epitopes from cells) both confirmed the combined benefits of SA- and MP-labeled nanogels. Pretreating B16F10 melanoma cells with GSH significantly reduced the $\mathrm{IC}_{50}$ values for PMNG/DOX and PNG/DOX as the reduction-labile nanogels decomposed, releasing DOX (Figure 10). 


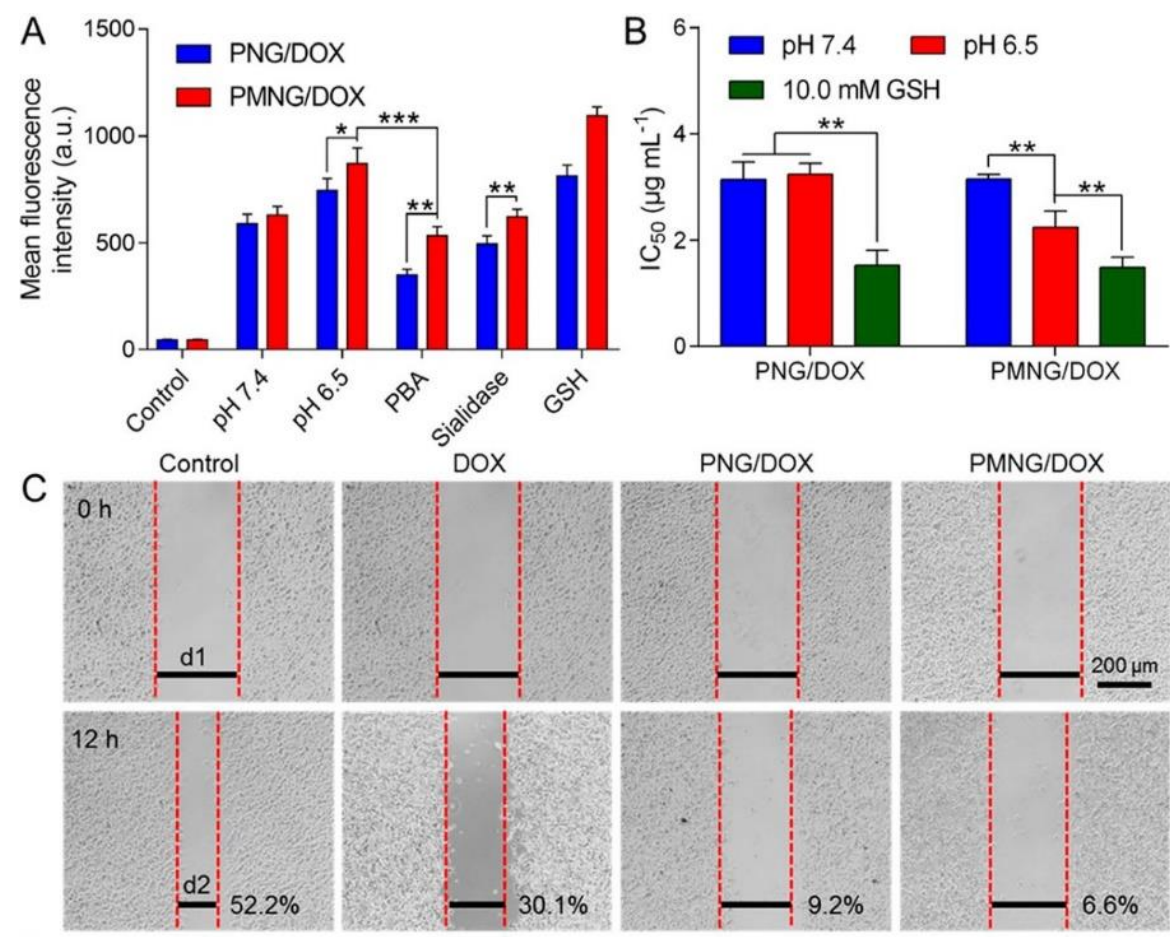

Figure 10. (A) cell viability studies were conducted with PMNG/DOX and PNG/DOX. As PNG/DOX lacks morpholine moieties it has lower affinity towards B16F10 cells at $\mathrm{pH}=6.5$, resulting in lower DOX uptake. When competition is introduced by sialidase, DOX uptake and thus fluorescence is decreased. (B) As PNG/DOX is not influenced by changes in $\mathrm{pH}$, its $\mathrm{IC}_{50}$ value remains the same at 7.4 as at 6.5 . PMNG/DOX, however increases in cytotoxicity at $\mathrm{pH}=6.5$, resulting in lower $\mathrm{IC}_{50}$. (C) Healing assays were conducted to assess the influence of PMNG/DOX and PNG/DOX on migration. After $12 \mathrm{~h}$ PNG/DOX allowed 9.2\% healing, whereas PMNG/DOX permitted 6.6\%. ${ }^{148}$ Reprinted (adapted) with permission from Ref ${ }^{148}$. Copyright 2017 American Chemical Society.

Metastasis is the leading cause of death in cancer patients as metastatic cells are highly efficient at migration and invasion. ${ }^{153-155}$ The authors probed the antimetastatic abilities of PMNG/DOX by conducting a wound healing assay B16F10 cells. Cells treated with PMNG/DOX exhibited the lowest migration distance. 


\subsubsection{Hyaluronic acid nanogels in cancer treatment}

The group of Zhong utilized HA nanogels to transport protein drugs cytochrome $\mathrm{C}(\mathrm{CC})$ and granzyme B (GrB) in vivo. ${ }^{54}$ The authors synthesized the HA nanogels (HA NGs) by means of an inverse nanoprecipitation followed by tetrazole-alkene photoclick reaction of HAcystamine-methacrylate (HA-Cys-MA) and HA-lysine-tretrazole (HA-Lys-Tet). HA exhibits intrinsic CD44 targeting, which is present on the cell membranes of human breast cells (MCF-7), lung tumor cells (A549), human multiple myeloma (LP1) and acute myelogenous leukemia (ALM2). ${ }^{156-158}$ The nanogel structure contains a pyrazoline functionality, making it intrinsically fluorescent with an emission wavelength of $\lambda=450 \mathrm{~nm} \cdot{ }^{159}$ Cystamine renders the nanogel responsive to a reducing environment. The authors demonstrated that HA NGs loaded with CC (CC-NGs) were preferentially taken up by CD44overexpressing MCF-7 cells and exhibited significantly higher cytotoxicity than in U87 CD44-free cancer cells, confirming receptor-mediated endocytosis (Figure 11a). HA NGs also successfully escaped the endosomes after $12 \mathrm{~h}$ of incubation. HA NGs loaded with GrB (GrB-NGs) demonstrated high potency against MCF-7 as well as A549 cancer cells (Figure 11c). 

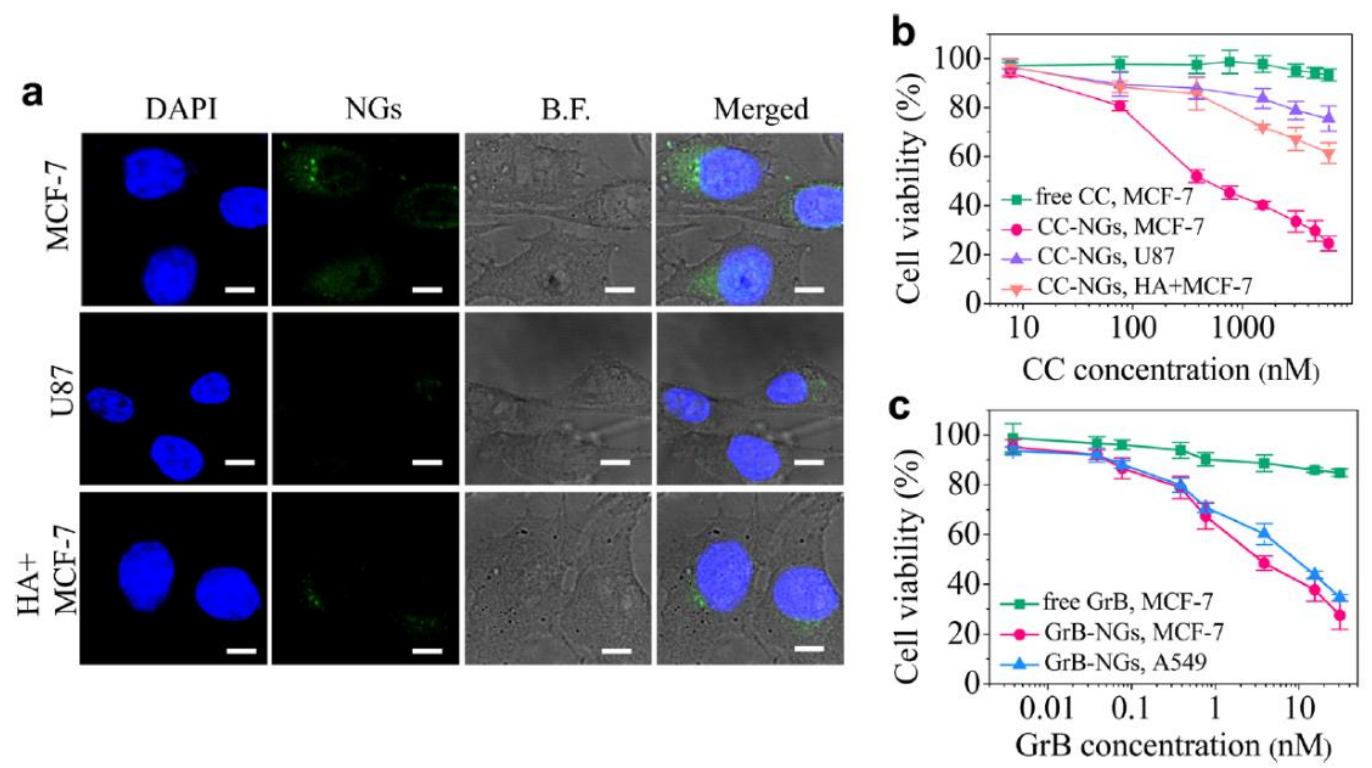

Figure 11. a) The cellular uptake of HA NGs was analyzed for MCF-7 cells that overexpress CD44 and U87 cells that do not, resulting in significantly higher uptake for MCF-7 cells. Introducing competition by first treating MCF-7 cells with HA, followed by HA NGs resulted in lower NG uptake, confirming CD44 targeting by the HA moiety. $b+c$ ) Cell viability was tested for CC-NGs and GrB-NGs on CD44 positive cells MCF-7 and A549 cells and on CD44 negative cells U87 cells. ${ }^{54}$ Reprinted (adapted) with permission from Ref ${ }^{54}$. Copyright 2016 American Chemical Society.

GrB-NGs were injected intravenously every 3 days in nude mice bearing MCF-7 tumors. Complete tumor suppression was achieved after 14 days when treated with $3.8 \mathrm{nmol}$ $\mathrm{GrB} / \mathrm{kg}$; a dosage 1000 lower than conventional chemotherapy drugs such as DOX, docetaxel and paclitaxel. ${ }^{160-164}$ Similar results were obtained for treatment of mice bearing A549 tumors with GrB-NGs. Complete suppression was achieved at $5.7 \mathrm{nmol} \mathrm{GrB} / \mathrm{kg}$.

Weng and Gu targeted nanogels to human cervical carcinoma epithelial (HeLa) cells by surface decoration with endosomal membrane proteins specific to HeLa cells. ${ }^{165}$ The authors devised a unique system to allow endocytosis of the materials needed to form the nanogel in situ in the target cell. A silica nanoparticle containing $\mathrm{Fe}_{3} \mathrm{O}_{4}$ was 
loaded with a crosslinker and photoinitiator followed by coating with HA to target CD44 receptors on cancer cells. This nanoparticle was then taken up by HeLa cells through endocytosis. Following irradiation with UV light, a photo-polymerization reaction took place synthesizing membrane-coated nanogels (EM-NGs). The $\mathrm{Fe}_{3} \mathrm{O}_{4}$ particles render the nanogel magnetic, making the nanogel extraction straightforward and simple. Western blotting confirmed the presence of endosomal membrane proteins on the nanogel surface. The authors loaded the EM-NGs with DOX and assessed their therapeutic effect on HeLa and A549 cancer cells. Enhanced DOX release behavior was observed at acidic pH resulting from the weakening of the DOX-nanogel bonds. The authors noted that various uptake pathways were involved in the endocytosis of DOX-EM-NGs, namely both clathrinand caveolin-mediated endocytosis, lipid rafts and macropinocytosis. DOX-EM-NGs exhibited the lowest cell viability in HeLa cells compared to free DOX and DOX-loaded bare nanogels (DOX-NGs) free of targeting ligands. A549 cells however did not exhibit significant difference in viability for cells treated with targeted DOX-EM-NGs and untargeted DOX-NGs. It can thus be concluded that synthesizing the nanogels in the cell of interest coats the nanogels with membrane proteins specific to that cell line-achieving active targeting.

It remains a challenge to effectively treat both primary cancer tumors as well as metastatic lesions with a singular delivery system. RHAMM is a HA receptor present in the tumor cells of stomach cancer ${ }^{166}$, prostate cancer ${ }^{167}$, leukemia ${ }^{168}$, breast cancer ${ }^{169}$, lung cancer ${ }^{170}$ and colorectal cancers ${ }^{171}$. CD44 is also a HA receptor present on the cell membrane of many cancers; however, certain tumors overexpress RHAMM and not CD44 meaning solely targeting CD44 is not sufficient. ${ }^{172}$ Jiang and coworkers utilized the reduction sensitivity of disulfide to construct $\mathrm{pH}$-sensitive HA nanogels, denoted HAss nanogels. ${ }^{173}$ As comparison nanogels were synthesized in a similar fashion but lacking a disulfide moiety (HAcc). HAss was loaded with DOX by means of electrostatic interactions 
between the amines of DOX and the carboxylate ions of HA. Cytotoxicity experiments were conducted on human prostate cancer (LNCaP), murine hepatoma (H22), human pulmonary cancer (A549) cells and fibroblasts (NIH3T3). Higher cytotoxicity was observed for free DOX in A549 and NIH3T3 cells compared to DOX-HAss. In contrast, DOX-HAss displayed higher cytotoxicity in $\mathrm{H} 22$ cells and similar cytotoxicity as free DOX in LNCaP cells. Higher cytotoxicity was achieved by HAss nanogels in cells that overexpress RHAMM (H22 and LNCaP) indicating an uptake pathway mediated by RHAMM. Uptake studies likewise indicated a higher uptake in H22 and LNCaP cells compared to NIH3T3. To further demonstrate that HAss nanogels are taken up by a RHAMM-mediated uptake pathway, a competition assay was carried out. When $\mathrm{H} 22$ and LNCaP cells were treated with antibodies against RHAMM, the uptake of HAss nanogels decreased significantly. Also, no co-localization can be seen for HAss nanogels and anti-RHAMM antibodies, indicating a competitive relationship between the two. Lastly, when HAss is labeled green and endosomes are stained red, co-localization is witnessed - suggesting uptake by endocytosis. In vivo experiments demonstrated deep tumor penetration and accumulation of HAss nanogels in metastatic lymph nodes.

\subsubsection{2 pH-responsive, DOX-delivering nanogels in cancer treatment}

The aforementioned strategy utilized by the group of Jiang, is a widely popular method seen throughout literature, including for non-HA-NGs. Many authors have synthesized redox-responsive nanogels that release $\mathrm{DOX}$ at acidic $\mathrm{pH}$ mimicking the tumor microenvironment. Pikabea et al. utilized poly(2-(diethylamino)ethyl methacrylate) (PDEAEMA) nanogels to this end. ${ }^{174}$ They successfully reduced the cell viability of HeLa cells and MDA-MB-231 breast cancer cells by up to $40 \%$ and demonstrated a dosedependent cytotoxicity. Asadi and Khoee synthesized a random copolymer of 
polyethylene glycol methyl ether methacrylate and 2-(pyridin-2-yldisulfanyl)ethyl acrylate (PDS-MPEG) and crosslinked it to a nanogel. ${ }^{55}$ DOX-loaded PDS-MPEG nanogels displayed higher cytotoxicity than free DOX in A2780CP ovarian carcinoma cells. Hang et al. engineered HA-CM nanogels consisting of $\mathrm{HA}$ and coumarin (CM) to target the CD44 receptors of MCF-7 cells. ${ }^{175}$ Coumarin is photoactive to both UV and NIR radiation. Irradiating DOX-loaded HA-CM nanogels for $60 \mathrm{~min}$ at $\lambda=780 \mathrm{~nm}$ decreased cell viability of MCF-7 cells by $50 \%$. U87MG glioblastoma cells that do not express CD44 were inhibited by approximately $15 \%$. The group of Zhang utilized pH-responsive, PEGylated liposomecoated pDMAEMA nanogels to deliver DOX to Hep-G2 and 4T1 cells. ${ }^{176}$ The DOX-loaded nanogels exhibited similar cytotoxicity as free DOX but were taken up less in the cancer cells, suggesting higher efficiency.

\subsubsection{Nanogels in (sub)cutaneous treatment}

Treating diseases topically is highly desired as it elicits great patient compliance. The skin however remains a challenging area to penetrate as it contains both hydrophilic and hydrophobic regions. ${ }^{177}$ Nanogels are proven to enhance drug delivery of both hydrophilic and lipophilic drugs with otherwise low solubility and absorption. The use of nanogels to enhance skin penetration has also been proven to be successful. ${ }^{178-181}$ The group of Kleuser set out to investigate the applicability of thermoresponsive polyglycerol nanogels (tPG) as drug delivery vehicle for passage through the skin. ${ }^{182}$ The epidermis consists primarily of dendritic cells; more specifically Langerhans cells. As such nanogels should be compatible with these cells and not elicit an immune response. The nanogel was synthesized from poly(glycidyl methyl ether-co-ethyl glycidyl ether) co-polymer (pGMEco-EGE) and dendritic polyglycerol (dPG). tNG_dPG_tPG nanogel exhibits a tunable cloud point temperature $\left(T_{c p}\right)$, relating to the ratio between GME and EGE. A ratio of 1:1 was 
chosen bestowing tNG_dPG_tPG $(1: 1)$ with a $T_{c p}$ of $29^{\circ} \mathrm{C}$. Cell viability studies with XS52 Langerhans cells determined that $t N G$ _dPG_tPG $G_{(1: 1)}$ and its IDCC-dyed equivalent (tNG_dPG_tPG $\left.{ }_{(1: 1)}-I D C C\right)$ are not toxic and exhibit high biocompatibility. Treatment with both nanogels did not generate reactive oxygen species by XS52 cells or damage its DNA. Uptake studies at $4^{\circ} \mathrm{C}, 29^{\circ} \mathrm{C}$ and $37^{\circ} \mathrm{C}$ exhibited steady increase over time, except at $4^{\circ} \mathrm{C}-$ suggesting an energy-dependent uptake mechanism. The authors determined with an inhibition assay that the main uptake pathway of $t N G_{-} d P G_{-} t P G_{(1: 1)}-I D C C$ by $L C s$ at $37^{\circ} \mathrm{C}$ was caveolae-mediated endocytosis. At $29^{\circ} \mathrm{C}$ however, both caveolae-mediated endocytosis and macropinocytosis were involved in cellular uptake. The authors hypothesized that the different uptake mechanisms at varying temperatures is a result of a phase transition. $t N G \_d P G \_t P G_{(1: 1)}-I D C C$ has a $T_{c p}$ of $29^{\circ} \mathrm{C}$, meaning that at temperatures $<29^{\circ} \mathrm{C}$ the nanogel is hydrophilic and above $29^{\circ} \mathrm{C}$ the nanogel will shrink, becoming more hydrophobic. At $29^{\circ} \mathrm{C}$ tNG_dPG_tPG $(1: 1)^{-}-\mathrm{IDCC}$ is equally hydrophilic as hydrophobic. Macropinocytosis involves the uptake of dissolved particles in biological media; requiring the particle to be hydrophilic. Contrarily, caveolae-mediated endocytosis involves the uptake of hydrophobic particles. As such tNG_dPG_tPG $(3: 1)$ possessing a $T_{c p}$ of $48^{\circ} \mathrm{C}$ was synthesized and its uptake mechanism was assessed at different temperatures. Indeed tNG_dPG_tPG $(3: 1)-I D C C$ was taken up by macropinocytosis at $29^{\circ} \mathrm{C}$ and $37^{\circ} \mathrm{C}$; indicating that it is hydrophilic at these temperatures, and thus confirming an influence of the $T_{c p}$ of the nanogel.

The team of Kleuser also evaluated the drug loading efficiency of thermoresponsive nanogel tNG_dPG_pNIPAM for hydrophobic cutaneous drugs dexamethasone and tacrolimus that treat severe skin inflammation and psoriasis. ${ }^{183}$ tNG_dPG_pNIPAM was made by crosslinking dPG with poly(N-isopropylacrylamide) (pNIPAM). ${ }^{184}$ Both tNG_dPG_tPG-IDCC and tNG_dPG_pNIPAM-IDCC were successfully taken up by normal human keratinocytes (NHK) and aneuploid immortal keratinocyte cells 
( $\mathrm{HaCaT}$ ) as depicted in Figure 12a and b. Colocalization of the nanogels with lysosomes after $24 \mathrm{~h}$ and $48 \mathrm{~h}$ suggests that the nanogels most likely do not escape the lysosomes. Cytotoxicity studies indicated no toxicity in NHK cells from either nanogel up to $0.5 \mathrm{mg} / \mathrm{mL}$ for $48 \mathrm{~h}$.
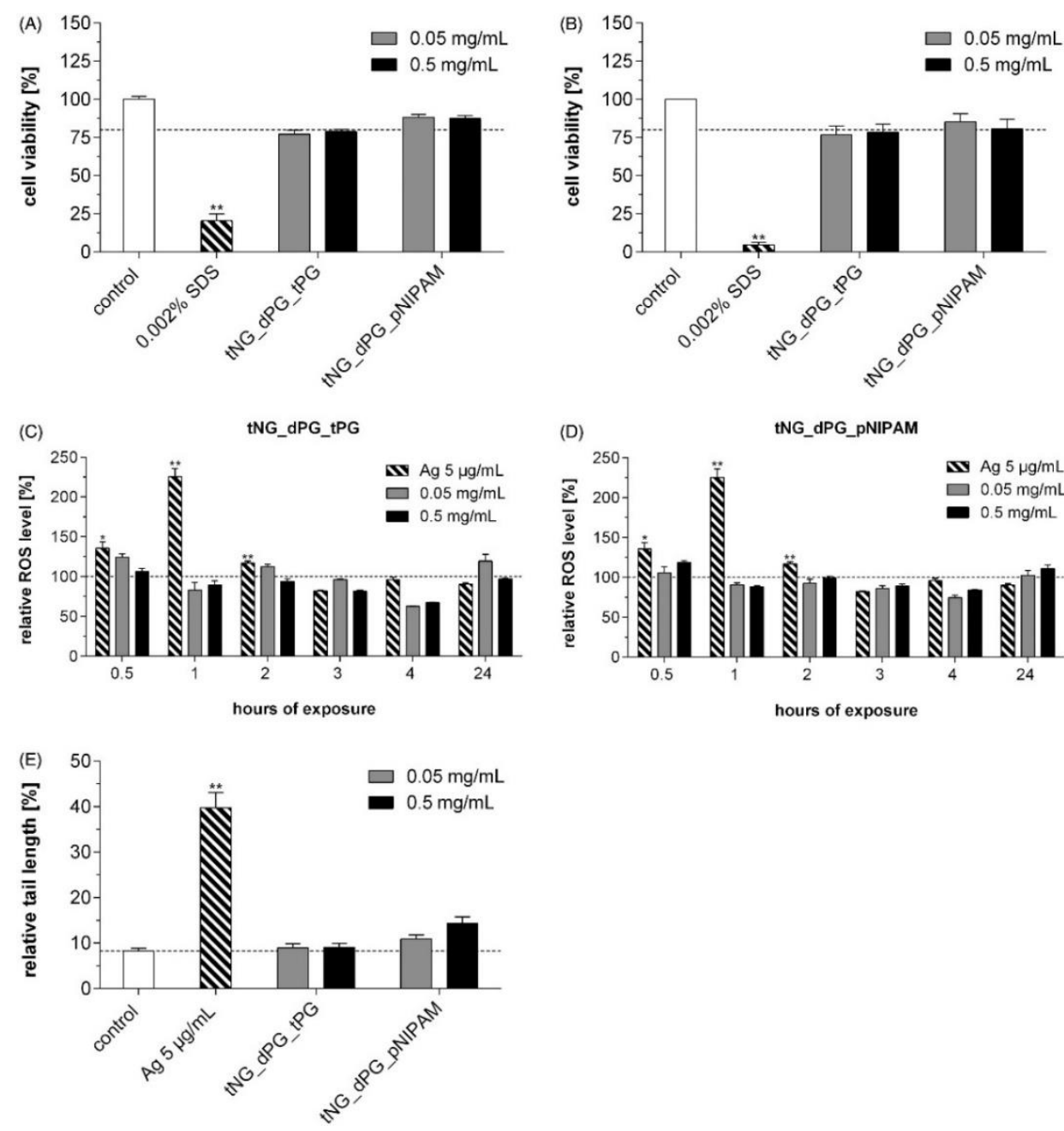

Figure 12. Cytotoxicity studies conducted with NHK cells for $24 \mathrm{~h}(\mathrm{~A})$ and $48 \mathrm{~h}(\mathrm{~B})$ determined that the nanogels were non-toxic up to $0.5 \mathrm{mg} / \mathrm{mL}$. Neither tNG_dPG_tPG (C) nor tNG_dPG_pNIPAM (D) exhibited ROS level increase for up to $24 \mathrm{~h}$. Silver nanoparticles served as positive control. (E) The nanogels did not exhibit genotoxic behavior up to $0.5 \mathrm{mg} / \mathrm{mL}$. Silver nanoparticles served as positive control. ${ }^{183}$ Reproduced with permission from Taylor \& Francis. 
The authors also evaluated the presence of reactive oxygen species (ROS) generated by the nanoparticles (Figure $12 c+d$ ). ${ }^{185}$ Likewise, evidence of genotoxicity stemming from nanoparticles has been shown. ${ }^{186-187}$ A carboxy-H2DCFDA assay and comet assay demonstrated no increase in ROS levels nor DNA damage by tNG_dPG_tPG nor tNG_dPG_pNIPAM. To complete the toxicology evaluation eye irritation tests were conducted. A bovine corneal opacity and permeability (BCOP) test as well as a hemolysis assay both determined that the nanogels were not irritating to mammalian eyes.

\subsection{Nanogels for peptide delivery}

Zhou et al. exploited the stimuli-responsive feature of nanogels to synthesize bloodcompatible nanogels that release insulin as a response to glucose concentrations. ${ }^{188} \mathrm{~A}$ monomer based on aminophenylboronic acid (AAPBA) was copolymerized with methacrylic acid (MAA) in a single step to form uniform, spherical nanogels. Aminophenylboronic acid shows reduction of the immune response in vivo. ${ }^{189}$ Nanogels Glu (1/4) and Glu (2/3) were made containing different AAPBA to MAA ratios. Both nanogels increase in size upon exposure to glucose with resulting release of insulin as a function of glucose concentration. Glu (1/4) and Glu (2/3) both demonstrated good blood compatibility up to $10 \mathrm{mg} / \mathrm{mL}$. Red blood cells were incubated with Glu (1/4) and Glu (2/3) at $0.01-10 \mathrm{mg} / \mathrm{mL}$ and their morphology was assessed by TEM. No aggregation or obvious morphological changes were apparent; most likely due to the negatively charged nanogels and blood cells repelling each other. Likewise, the nanogels did not interact with coagulation factors in blood plasma. Lastly, cytotoxicity studies with Glu (2/3) in 3T3 cells (mouse embryonic fibroblasts) over $24 \mathrm{~h}$ and $48 \mathrm{~h}$ indicated high cell viability up to 100 $\mu \mathrm{g} / \mathrm{mL}$. 
In section 2.11.1 we highlighted functional nanogels for cancer treatment with (chemotherapeutic) drugs. Anticancer activity can also be achieved by proapoptotic peptides such as $(\text { KLAKLAK })_{2}$. (KLAKLAK $)_{2}$ induces apoptosis by interacting with the mitochondria of tumor cells. ${ }^{190-191} \mathrm{Oh}$ and Lee utilized $\mathrm{pH}$-responsive polysaccharide nanogels comprising of chitosan, starch and 3-diethylaminopropylamine (DEAP) to deliver $\left(\right.$ KLAKLAK) 2 to the cytosol of KB tumor cells. ${ }^{192}$ DEAP is uncharged at $\mathrm{pH} 7.4$ but becomes cationic at acidic pH; resulting in the expulsion of positively charged (KLAKLAK) 2 . The authors first activated starch with succinic anhydride, followed by reactions with chitosan (GC) and DEAP, producing starch-(GC-DEAP). Starch-(GC-DEAP) self-assembled to coreshell nanogels in the presence of D-(KLAKLAK) $)_{2}$ loading the peptide with $50 \%$ efficiency. The hydrophilic shell contained the GC moieties and the hydrophobic core consisted of neutral DEAP. The cellular uptake of starch-(GC-DEAP) by human nasopharyngeal epidermal carcinoma (KB) cells was studied by CLSM (Figure 13).
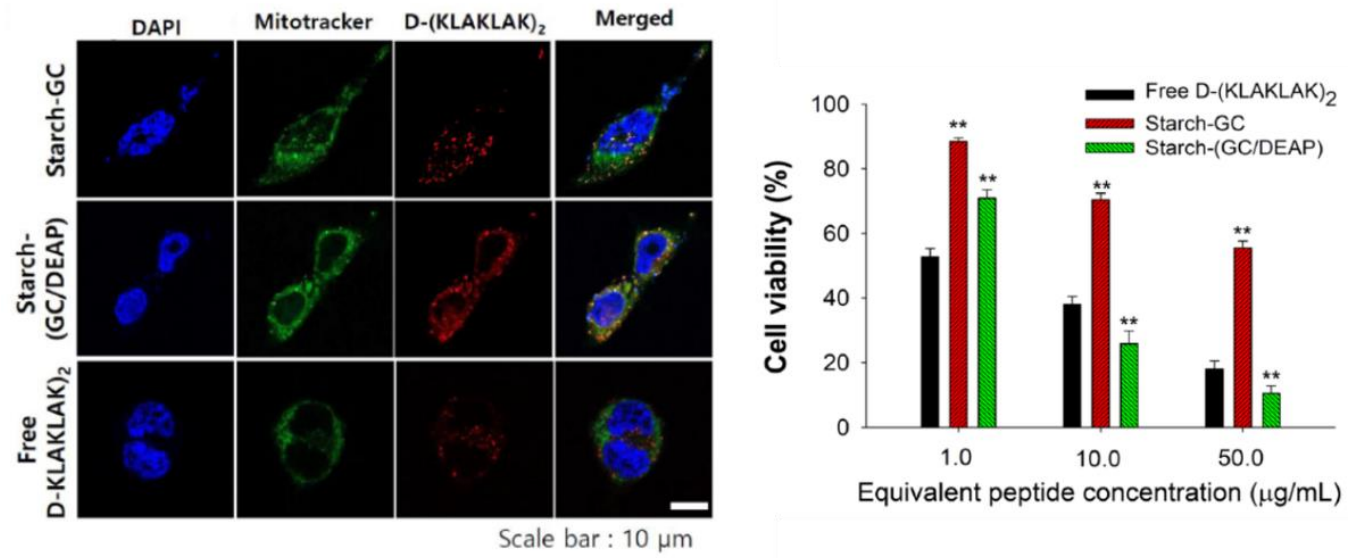

Figure 13. CLSM images taken after $4 \mathrm{~h}$ of incubation of KB cells with free (KLAKLAK) $)_{2}$, starch-(GC-DEAP) and starch-GC indicate high amounts of (KLAKLAK) ${ }_{2}$, in the mitochondria. Cell viability studies demonstrate the utility of starch-(GC-DEAP) as peptide carrier at various concentrations. ${ }^{192}$ Reproduced and adapted with permission from SAGE Publications. 
Starch-(GC-DEAP) achieved the highest colocalization of (KLAKLAK) $)_{2}$ in the mitochondria of the KB cells, compared to free (KLAKLAK) ${ }_{2}$ and starch-GC. Likewise, starch-(GC-DEAP) exhibited significant hemolysis at endosomal pH 6 . Cell viability studies indicated that starch-(GC-DEAP) was the most effective peptide delivery vehicle; exhibited the highest $\mathrm{KB}$ cell death at $10 \mu \mathrm{g} / \mathrm{mL}$ or higher.

\subsection{Nanogels for gene delivery}

Various diseases originate in the genome; either by gene deletions, gene insertions, gene overexpression or gene dysregulation. Some examples being cancers, cystic fibrosis, sickle cell anemia, muscular dystrophy and other (viral) infections and hereditary conditions. Treating these diseases at their genetic source increases the chance of recovery. Applying gene therapy techniques such as gene silencing can inhibit gene expression by utilizing small interfering RNA (siRNA) molecules. ${ }^{193}$ The group of Haag formed $\mathrm{pH}$ sensitive nanogels from dendritic polyglycerol (dPG) and polyethyleneimini (PEI) to afford cationic transfection vectors. ${ }^{194} \mathrm{By}$ incorporating a $\mathrm{pH}$-sensitive benzacetal unit, control is gained over the in vitro cargo release of the nanogel. The authors opted to load the nanogels with RNA by forming polyplexes (NGp) i.e. mixing nanogels with RNA, and by synthesizing the nanogels in the presence of RNA resulting in RNA-encapsulated nanogels (NGe). Cytotoxicity studies in GFP-expressing HeLa cells indicated higher cell viability for NGp at higher concentrations than NGe; most likely due to NGe having the majority of its surface cationic charge unmasked. Gene silencing experiments were conducted with GFPexpressing HeLa cells, NGe, NGp and PEI. NGe and NGp exhibited similar transfection efficiencies of $72 \%$. PEI displayed a higher efficiency of $83 \%$; however, as PEI is known for its high toxicity NGe and NGp make great alternatives. 
Gene transfection is another subdivision of gene therapy aimed at treating genetic diseases. As opposed to inhibiting the expression of disease causing genes, transfection experiments aim to introduce foreign or synthetic nucleotides into the human body to express useful genes and proteins. These nucleotides, often plasmid DNA, require targeted and safe delivery to the cell nucleus. Nanogels make interesting transfection gene vectors owing to their versatility, attractive properties and tunability. Park et al. synthesized sunflower type nanogels for the internalization of quantum dots (QDs) to function as gene delivery vehicles as well as in vitro imaging agents. ${ }^{195}$ The nanogel construct was made up of heparin and pluronic F127. Heparin is an anticoagulant and pluronic F127 is a triblock copolymer and surfactant. QDs containing an amine functionality were loaded into the nanogels, followed by coating of the nanogel surface with PEI. The cationic PEl coating influenced the position of the QDs in the nanogel by means of charge interactions. As both are positively charged, QDs were driven to the center of the nanogels. The nanogels were incubated with human mesenchymal stem cells (hMSCs) and their uptake and cell distribution was followed via the enclosed QDs. TEM images display the nanogels moving from the cell membrane, to early endosomes, maturing to late endosomes, before ending up at the cell nucleus. Sunflower-type nanogels outperformed PEI in transfection studies by exhibiting higher GFP fluorescence. FACS measurements indicated a higher cell uptake for a nanogel to QD ratio of 1:2. However, the higher transfection efficiency was achieved at a ratio of 1:0.25 (Figure 14). 

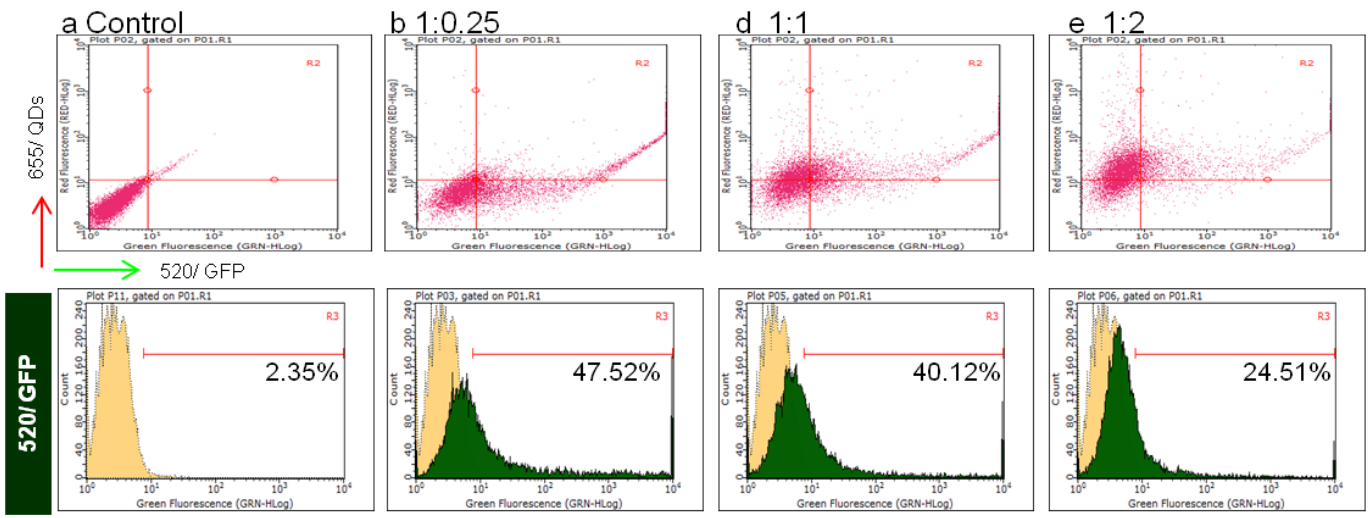

Figure 14. FACS measurements of the cell uptake (top row) and GFP expression (bottom row) of nanogels encapsulating various amounts of QDs. ${ }^{195}$ Reproduced and adapted with permission from Elsevier.

HA nanogels that target CD44 remains an established method seen in drug and gene delivery. Park and coworkers demonstrated its versatility by delivering SOX9 gene to human mesenchymal stem cells (hMSCs) in order to regenerate cartilage. ${ }^{196}$ The gene transfection efficiency of PEI is well documented, as well as its intrinsic toxicity resulting from the high cationic charge. ${ }^{197}$ Shielding this charge when needed and selectively exposing it to enhance transfection would provide the researcher with the best of both worlds. Park and coworkers utilized a simple strategy to synthesize nanogels, involving first mixing PEI with pDNA, followed by coating with FITC-labeled HA. Various PEI to HA ratios were tested during uptake studies, with 1:10 PEl:HA exhibiting the highest uptake. When anti-CD44 antibodies were present, uptake of the nanogels was significantly lower - proving CD44-mediated uptake. In order to visualize and quantitate transfection, SOX9 gene was tagged with EGFP resulting in green fluorescence for successful transfection. Sustained fluorescence was observed for up to $72 \mathrm{~h}$.

Li et al. probed the gene transfection efficiency of poly(glycidyl methacrylate) (pGMA) modified with ethylenediamine to treat hepatomas. ${ }^{198}$ Making use of the 
reduction potential difference between regular cells and cancer cells, the authors opted for a pH-responsive nanogel. Utilizing disulfide bonds is a creative way to ensure cargo release at acidic $\mathrm{pH}$ as well as biodegradability. As such disulfide-containing $\alpha$-lipoic acid (LA) was employed as biocompatible crosslinking agent to synthesize reducible cationic nanogels. Linear PGMA was first synthesized by ATRP polymerization, followed by ringopening amination with ethylenediamine to form PGED. Lastly, PGED was subjected to amidation with LA and crosslinked to nanogels (PGED-NG). Two sets of nanogels were made with different molecular weights, PGED1-NG and PGED2-NG. Each set contained three nanogels with varying amounts of lipoyl groups and thus various crosslink densities. PGED2 had a higher molecular weight and could effectively condense pDNA at N/P $=2$, whereas PGED1 required an N/P ratio of 2.4 or higher. Cytotoxicity studies in HEK293, COS7 and HepG2 cells demonstrated a minimum of $85 \%$ cell viability for all nanogel formulations, up to N/P = 50. Gene transfection experiments displayed an apparent effect of crosslink density and molecular weight on transfection efficiency. Nanogels containing higher crosslink densities consistently achieved higher luciferase expression. The same trend was seen for molecular weights. Interestingly, PGED2-NG20, containing 20\% crosslink density, outperformed $25 \mathrm{kDa}$ PEI in all cell lines, including HepG2 cells that are notoriously difficult to transfect. The internalization fractions were calculated for the transfected HepG2 cells, comparing nanogels to their cationic polymer equivalents. PGED1 had an internalization fraction of $44 \%$ whereas $65 \%$ of HepG 2 cells endocytosed PGED2 polyplexes. The nanogel counterparts possessing $20 \%$ crosslink densities exhibited internalization fractions of $75 \%$ for PGED1-NG20 and $86 \%$ for PGED2-NG20. The authors next evaluated the knockdown efficiencies of PGED1-NG20 and PGED2-NG20 in gene silencing of MALAT1 present in cell carcinoma (Figure 15). ${ }^{199}$ PGED1-NG20 exhibited the highest decrease in MALAT1 expression compared to negative control, with accompanying highest inhibition of cell proliferation. 

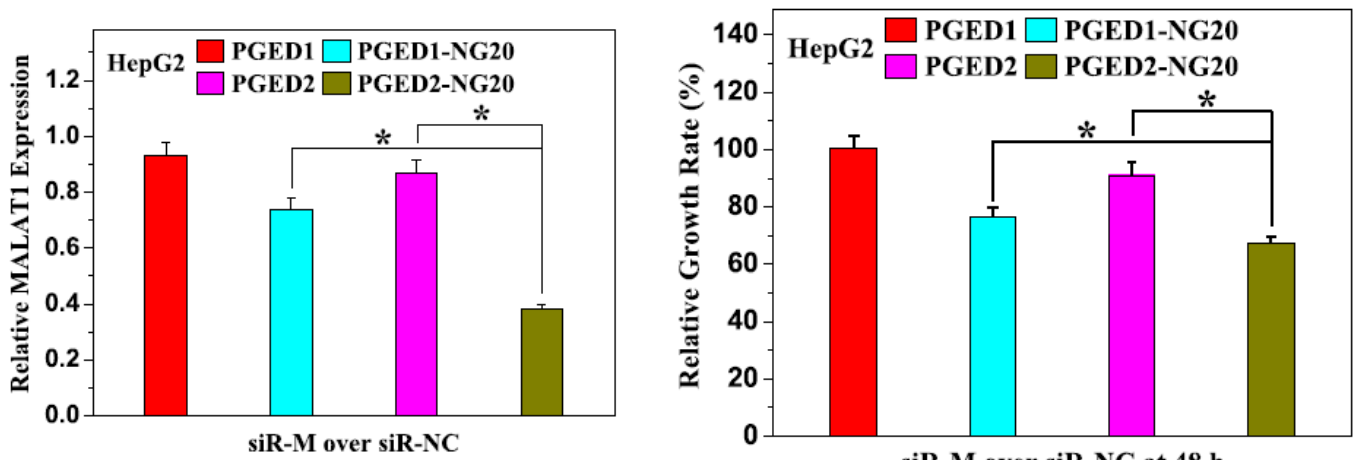

Figure 15. Gene silencing efficiencies of PGED1 and PGED2 cationic polymers as well as PGED1-NG20 and PGED2-NG20 nanogels (siR-M) are given as a ratio to negative control (siR-NC), depicted on the left. PGED2NG20 exhibited the highest inhibition of MALAT1 gene expression with corresponding decrease in cell growth rate, depicted in the chart on the right. ${ }^{198}$ Reproduced and adapted with permission from Elsevier.

\subsection{Nanogels for protein delivery}

Protein therapy as a treatment option for various diseases is gaining traction. ${ }^{200}$ In order to ensure efficiency, proteins must be shielded from biological media to avoid unwanted occurrences such as denaturation. Peng and coworkers employed $\mathrm{pH}$-responsive nanogels to delivery bovine serum albumin (BSA) to HeLa cells. ${ }^{201}$ The core-shell nanogel was engineered by enzyme-oxidative coupling of the tyramine moieties in PEG- $b$-P(LGA$g$-Tyr) polymer by horseradish peroxidase (HRP). The nanogel exhibited an initial burst release of BSA (from the PEG shell) followed by consistent prolonged release for $45 \mathrm{~h}$ from the nanogel core. As expected, BSA release was higher at acidic pH 6.8 than at physiological pH 7.4. Neither empty nanogels nor BSA-loaded nanogels exhibited cytotoxicity towards HeLa cells - even up to $2 \mathrm{mg} / \mathrm{mL}$. BSA-loaded nanogels were successfully taken up by HeLa cells at pH 7.4 and 6.8; displaying higher endocytosis at acidic $\mathrm{pH}$, owing to its smaller size. Free FITC-labeled BSA exhibited minimal cell uptake. 
Granzyme B (GzmB) is a naturally occurring cytotoxic serum protein taking part in the innate immune system. Utilizing cytotoxic proteins such as cytochrome c, caspase-3, RNase $A$ and p53 recombinant protein to treat cancer has gained interest in recent years. ${ }^{202-207}$ Kurisawa and coworkers encapsulated GzmB into nanogels to target and deliver it to HCT-116 colon cancer cells. ${ }^{208}$ The nanogels were formed through selfassembly and contained HA to target CD44-overexpressed cancer cells and enhance uptake via endocytosis. A conjugate of epigallocatechin-3-gallate (EGCG) was incorporated into the nanogel design to exert anticancer and antioxidant effects as well as bind GzmB. ${ }^{209-212}$ The cationic nature of linear polyethylenimine (PEI) was exploited to ensure endosomal escape of GzmB. HA-EGCG/GzmB nanogel lacking PEI did not succeed in exerting cytotoxic behavior. The anticancer properties of HA-EGCG/GzmB/PEI nanogel were tested on HCT-116 cells. GzmB induces apoptosis of cells by first activating caspase3 and caspase-7 enzymes. ${ }^{213}$ HCT-116 cells were incubated with various nanogel formulations and their expression of caspase-3 and 7 were calculated. HAEGCG/GzmB/PEI nanogel demonstrated the highest caspase activity, making it the most cytotoxic to HCT-116 cells.

Hydroxyapatite ( $\left.\mathrm{HAP}, \mathrm{Ca}_{5}\left(\mathrm{PO}_{4}\right)_{3}\right)$ is a crystalline naturally occurring mineral often used in dentistry and orthopedics. ${ }^{214-215}$ It has also found purpose as biomolecular carrier for proteins such as BSA. ${ }^{216}$ To achieve higher protein delivery and stability, hybrid HAP nanogel particles were synthesized from polyacrylic acid (PAA) and chitosan (CS). ${ }^{217}$ PAA stabilizes calcium phosphate and CS enhances biocompatibility. ${ }^{215,} 218$ Nanogels were synthesized with a 1:1 ratio between PAA and CS. The nanogels were subsequently loaded with HAP by stirring with $0.5 \mathrm{M} \mathrm{Ca}\left(\mathrm{NO}_{3}\right)_{2}$ and $\mathrm{NaH}_{2} \mathrm{PO}_{4}$ solution $(1: 1 \% / 6 \mathrm{Ca} / \mathrm{P})$. BSA was successfully adsorbed onto the nanogel surface showcasing a trend of decreasing amount of adsorbed BSA with increasing nanogel concentration. Cytotoxicity studies with rat bone marrow stromal stem cells (BMSC) exhibited exceptional cell viability even up to $8 \mathrm{mg} / \mathrm{mL}$ 
after 5 days. Likewise hemocompatibility studies indicated minimal hemolysis as red blood cells did not recognize the HAP nanogels as foreign objects.

\subsection{Nanogels as nanovaccines}

As opposed to chemotherapy, cancers can also be treated with vaccines. Ma and coworkers developed a nanovaccine with self-adjuvant properties. ${ }^{219}$ A zwitterionic polysaccharide nanogel was synthesized from galactosyl, dextran and a retinoid derived from vitamin A, to target dendritic cells. The galactosyl moiety renders the GDR nanogel $\mathrm{pH}$-sensitive whereas the hydrazone moiety binding the retinoid endows the nanogel with acid-sensitivity. Vitamin A increases vaccine efficacy making it a potent vaccine adjuvant. Cell uptake studies with bone marrow-derived dendritic cells (BMDC) demonstrated the increased uptake of OVA antigen when delivered by GDR nanogels. Introducing competition with galactose resulted in a decrease of GDR/OVA nanogel uptake, indicating active targeting by the galactosyl moiety (Figure 16a). A similar study in which acidizing inhibitor $\mathrm{NH}_{4} \mathrm{Cl}$ was used, confirmed acidity enhanced uptake as well as lysosomal rupture/escape (Figure $16 b+c$ ). Lastly, treatment of dendritic cells with GDR/OVA nanogel was studied. As seen in Figure $16 \mathrm{~d}+\mathrm{e}, \mathrm{GDR} /$ OVA nanogel increased the T cell proliferation for both MHC1 and MHC2 signifying an increase in immune response and thus excellent adjuvant properties. 
(A)

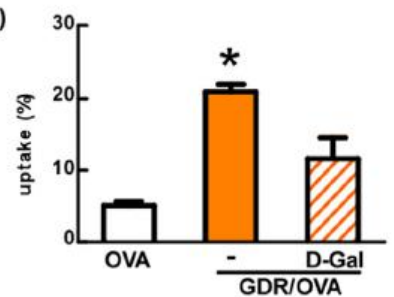

(C)
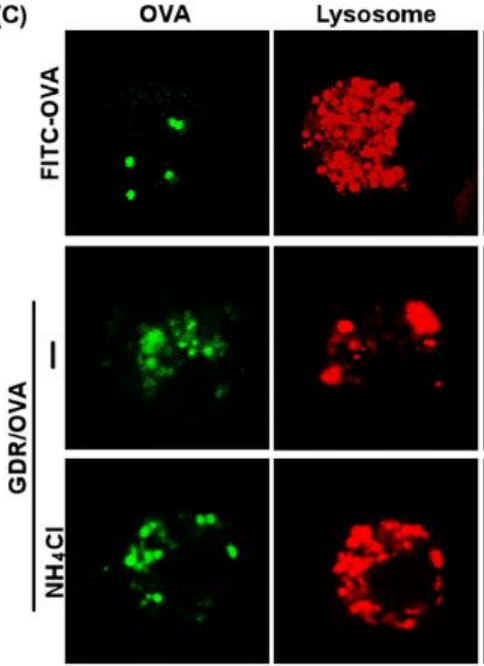

(D)

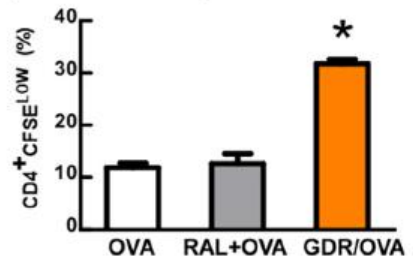

(B)
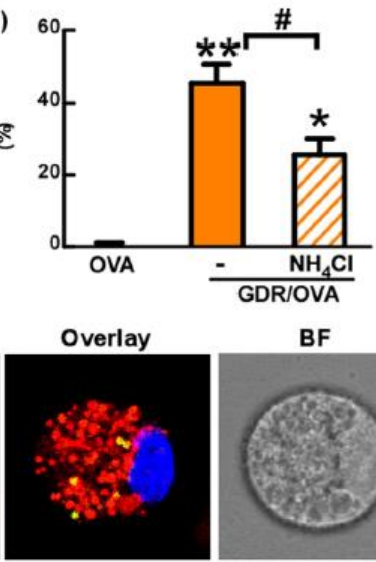

BF

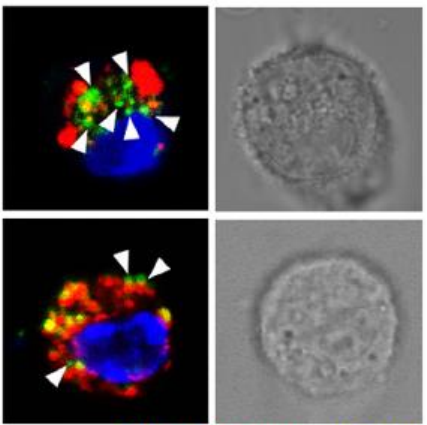

OVA / lysosome / nucleus

(E) MHC I presentation

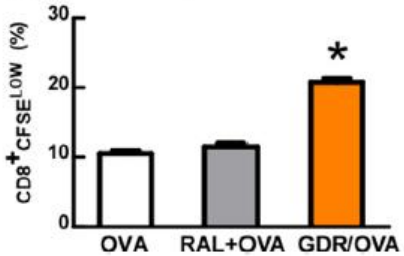

Figure 16. Cell uptake studies were conducted with GDR/OVA nanogels to evaluate active targeting. (A) Introducing competition with galactose lowered the uptake of GDR/OVA nanogel, confirming targeting by the galactosyl moiety. (B) Likewise, decreasing acidity resulting in lower uptake, confirming acidity enhanced uptake. (C) The highest uptake and lysosomal escape is witnessed for GDR/OVA nanogel in the absence of $\mathrm{NH}_{4} \mathrm{Cl}$. (D+E) GDR/OVA nanogel enhances CD4+ and CD8+ T cell production demonstrating adjuvant abilities for both MHC class 1 and $2 .{ }^{219}$ Reproduced with permission from Elsevier. 
Nuhn et al. studied agonists of Toll-like receptors TLR7 and TLR8 as vaccine adjuvants by covalently binding an imidazoquinoline equivalent (IMDQ) onto the surface of nanogels. ${ }^{220}$ Fixing the agonists on nanogels limits their rapid diffusion which in turn lowers their toxicity. ${ }^{221-222} \mathrm{pH}$-responsive self-assembled nanogels were synthesized from a methoxy triethylene glycol methacrylate (mTEGMA) pentafluorophenyl methacrylate (PFPMA) amphiphilic block copolymer. ${ }^{223}$ The mTEGMA-PFPMA nanogels were subsequently functionalized with IMDQ and crosslinked. The activation ability of IMDQligated nanogels was assessed on bone marrow-derived dendritic mouse cells. IMDQligated polymers were used as comparison. IMDQ-ligated nanogels successfully activated dendritic cells. However, free IMDQ exhibited higher levels of CD80 and MHC class II likely due to a decrease in IMDQ potency once modified. In vivo experiments demonstrated significantly lower diffusion of IMDQ when injected in the footpad of mice as IMDQligated nanogels. Administration of free IMDQ resulted in rapid systemic IFN- $\beta$ response signifying severe inflammatory toxicity. ${ }^{224-225}$ Unlike free IMDQ, IMDQ-ligated nanogels elicited a high innate immune response in the form of migration of granulocytes and monocytes to the inflammation site.

\subsection{Summary and conclusion}

Surface functionalization of nanogels is a relatively easy way to confer all sorts of properties to nanogels as well as increase their biocompatibility, targeting efficiency, uptake, hydrophilicity, tune their surface charge and enact many other desired changes. Nanogels allow functional group interconversions, conjugation of enzymes, PEG chains, sugars, proteins, DNA, drugs, peptides and antibodies without disrupting the morphology of the nanogel network. The majority of the conjugations were done through EDC/NHS coupling; however, functionalizations by "click" chemistry with maleimide and thiols were 
also demonstrated. Acid-labile hydrazone bonds and Schiff base linkages were also used to attach molecules of interest to the nanogels, followed by subsequent release at acidic $\mathrm{pH}$. Moreover, surface decoration was achieved by thiol-disulfide exchange, bio recognition and even enzymes. It can thus be concluded that surface functionalization of nanogels is relatively easy and straightforward.

Nanogels of appropriate size are easily internalized by various cell types, both malignant and benign. Functional nanogels for biomedical applications were often dualresponsive to maximize efficiency. Monomers, crosslinkers, cargo load and surface moieties were methodically chosen to incorporate multiple functionalities into the nanogel. Creative methods were employed to achieve endocytosis, such as nanogel synthesis in liposomes. Passive targeting was mostly observed for nanogels in cancer therapy that exploited EPR. The vast majority employed active targeting and even dualtargeting to achieve selectivity. As a result, cellular uptake was mostly carried out by receptor-mediated endocytosis. Instances of clathrin-mediated, caveolin-mediated, lipid raft-dependent and macropinocytosis were also observed. Nanogels show great potential in biomedical applications. Namely, anticancer treatment with nanogels has attained great successes both in vitro as well as in vivo - achieving cancer cell viability as low as $18 \%$.

\subsection{References}

1. Charman, W. N.; Chan, H. K.; Finnin, B. C.; Charman, S. A., Drug delivery: a key factor in realising the full therapeutic potential of drugs. Drug Development Research 1999, 46 (3-4), 316-327.

2. Torchilin, V. P., Structure and design of polymeric surfactant-based drug delivery systems. Journal of controlled release 2001, 73 (2-3), 137-172. 
3. Müller-Goymann, C., Physicochemical characterization of colloidal drug delivery systems such as reverse micelles, vesicles, liquid crystals and nanoparticles for topical administration. European Journal of Pharmaceutics and Biopharmaceutics 2004, 58 (2), 343-356.

4. Soppimath, K. S.; Aminabhavi, T. M.; Kulkarni, A. R.; Rudzinski, W. E., Biodegradable polymeric nanoparticles as drug delivery devices. Journal of controlled release 2001, 70 (1-2), 1-20.

5. Juliano, R. L.; Ming, X.; Nakagawa, O., Cellular uptake and intracellular trafficking of antisense and siRNA oligonucleotides. Bioconjugate chemistry 2012, 23 (2), 147-157.

6. Oh, J. K.; Lee, D. I.; Park, J. M., Biopolymer-based microgels/nanogels for drug delivery applications. Progress in polymer science 2009, 34 (12), 1261-1282.

7. Oh, J. K.; Drumright, R.; Siegwart, D. J.; Matyjaszewski, K., The development of microgels/nanogels for drug delivery applications. Progress in Polymer Science 2008, 33 (4), 448-477.

8. Steinhilber, D.; Rossow, T.; Wedepohl, S.; Paulus, F.; Seiffert, S.; Haag, R., A microgel construction kit for bioorthogonal encapsulation and $\mathrm{pH}$-controlled release of living cells. Angewandte Chemie International Edition 2013, 52 (51), 13538-13543.

9. Sharma, A.; Garg, T.; Aman, A.; Panchal, K.; Sharma, R.; Kumar, S.; Markandeywar, T., Nanogel-an advanced drug delivery tool: Current and future. Artificial cells, nanomedicine, and biotechnology 2016, 44 (1), 165-177.

10. Chacko, R. T.; Ventura, J.; Zhuang, J.; Thayumanavan, S., Polymer nanogels: a versatile nanoscopic drug delivery platform. Advanced drug delivery reviews 2012, 64 (9), 836-851.

11. Cao, Z.; Ziener, U., Synthesis of nanostructured materials in inverse miniemulsions and their applications. Nanoscale 2013, 5 (21), 10093-10107. 
12. Chaterji, S.; Kwon, I. K.; Park, K., Smart polymeric gels: redefining the limits of biomedical devices. Progress in polymer science 2007, 32 (8-9), 1083-1122.

13. Fujioka-Kobayashi, M.; Ota, M. S.; Shimoda, A.; Nakahama, K.-i.; Akiyoshi, K.; Miyamoto, Y.; Iseki, S., Cholesteryl group-and acryloyl group-bearing pullulan nanogel to deliver BMP2 and FGF18 for bone tissue engineering. Biomaterials 2012, 33 (30), 7613-7620.

14. Xia, Y.; He, X.; Cao, M.; Chen, C.; Xu, H.; Pan, F.; Lu, J. R., Thermoresponsive microgel films for harvesting cells and cell sheets. Biomacromolecules 2013, 14 (10), 3615-3625.

15. Yallapu, M. M.; Jaggi, M.; Chauhan, S. C., Design and engineering of nanogels for cancer treatment. Drug discovery today 2011, 16 (9-10), 457-463.

16. Dorwal, D., Nanogels as novel and versatile pharmaceuticals. Int J Pharm Pharm Sci 2012, 4 (3), 67-74.

17. Maya, S.; Sarmento, B.; Nair, A.; Rejinold, N. S.; Nair, S. V.; Jayakumar, R., Smart stimuli sensitive nanogels in cancer drug delivery and imaging: a review. Current pharmaceutical design 2013, 19 (41), 7203-7218.

18. Soni, G.; Yadav, K. S., Nanogels as potential nanomedicine carrier for treatment of cancer: A mini review of the state of the art. Saudi Pharmaceutical Journal 2016, 24 (2), 133-139.

19. Hasegawa, U.; Shin-ichiro, M. N.; Kaul, S. C.; Hirano, T.; Akiyoshi, K., Nanogelquantum dot hybrid nanoparticles for live cell imaging. Biochemical and biophysical research communications 2005, 331 (4), 917-921.

20. Wu, W.; Shen, J.; Banerjee, P.; Zhou, S., Core-shell hybrid nanogels for integration of optical temperature-sensing, targeted tumor cell imaging, and combined chemo-photothermal treatment. Biomaterials 2010, 31 (29), 7555-7566.

21. Hoare, T.; Young, S.; Lawlor, M. W.; Kohane, D. S., Thermoresponsive nanogels for prolonged duration local anesthesia. Acta biomaterialia 2012, 8 (10), 3596-3605. 
22. Zhao, C.; Chen, Q.; Patel, K.; Li, L.; Li, X.; Wang, Q.; Zhang, G.; Zheng, J., Synthesis and characterization of $\mathrm{pH}$-sensitive poly ( $\mathrm{N}$-2-hydroxyethyl acrylamide)-acrylic acid (poly (HEAA/AA)) nanogels with antifouling protection for controlled release. Soft Matter 2012, 8 (30), 7848-7857.

23. Bridges, A. W.; Singh, N.; Burns, K. L.; Babensee, J. E.; Lyon, L. A.; García, A. J., Reduced acute inflammatory responses to microgel conformal coatings. Biomaterials 2008, 29 (35), 4605-4615.

24. Kim, H.-J.; Zhang, K.; Moore, L.; Ho, D., Diamond nanogel-embedded contact lenses mediate lysozyme-dependent therapeutic release. ACS nano 2014, 8 (3), 2998-3005.

25. Wu, W.; Shen, J.; Li, Y.; Zhu, H.; Banerjee, P.; Zhou, S., Specific glucose-to-SPR signal transduction at physiological $\mathrm{pH}$ by molecularly imprinted responsive hybrid microgels. Biomaterials 2012, 33 (29), 7115-7125.

26. Wu, W.; Mitra, N.; Yan, E. C.; Zhou, S., Multifunctional hybrid nanogel for integration of optical glucose sensing and self-regulated insulin release at physiological pH. Acs Nano 2010, 4 (8), 4831-4839.

27. Li, C.; Liu, S., Responsive nanogel-based dual fluorescent sensors for temperature and $\mathrm{Hg} 2+$ ions with enhanced detection sensitivity. Journal of Materials Chemistry 2010, 20 (47), 10716-10723.

28. Zhu, H.; Li, Y.; Qiu, R.; Shi, L.; Wu, W.; Zhou, S., Responsive fluorescent Bi2O3@ PVA hybrid nanogels for temperature-sensing, dual-modal imaging, and drug delivery. Biomaterials 2012, 33 (10), 3058-3069.

29. Wu, W.; Shen, J.; Gai, Z.; Hong, K.; Banerjee, P.; Zhou, S., Multi-functional coreshell hybrid nanogels for $\mathrm{pH}$-dependent magnetic manipulation, fluorescent $\mathrm{pH}$ sensing, and drug delivery. Biomaterials 2011, 32 (36), 9876-9887. 
30. Peng, H. S.; Stolwijk, J. A.; Sun, L. N.; Wegener, J.; Wolfbeis, O. S., A nanogel for ratiometric fluorescent sensing of intracellular $\mathrm{pH}$ values. Angewandte Chemie 2010, $122(25), 4342-4345$.

31. Wu, W.; Aiello, M.; Zhou, T.; Berliner, A.; Banerjee, P.; Zhou, S., In-situ immobilization of quantum dots in polysaccharide-based nanogels for integration of optical pH-sensing, tumor cell imaging, and drug delivery. Biomaterials 2010, 31 (11), 3023-3031.

32. Nuhn, L.; Hirsch, M.; Krieg, B.; Koynov, K.; Fischer, K.; Schmidt, M.; Helm, M.; Zentel, R., Cationic nanohydrogel particles as potential siRNA carriers for cellular delivery. Acs Nano 2012, 6 (3), 2198-2214.

33. Nuhn, L.; Gietzen, S.; Mohr, K.; Fischer, K.; Toh, K.; Miyata, K.; Matsumoto, Y.; Kataoka, K.; Schmidt, M.; Zentel, R., Aggregation behavior of cationic nanohydrogel particles in human blood serum. Biomacromolecules 2014, 15 (4), 1526-1533.

34. Nuhn, L.; Tomcin, S.; Miyata, K.; Mailänder, V.; Landfester, K.; Kataoka, K.; Zentel, R., Size-dependent knockdown potential of siRNA-loaded cationic nanohydrogel particles. Biomacromolecules 2014, 15 (11), 4111-4121.

35. Zarekar, N. S.; Lingayat, V. J.; Pande, V. V., Nanogel as a novel platform for smart drug delivery system. Nanoscience and Nanotechnology 2017, 4 (1), 25-31.

36. Peer, D.; Karp, J. M.; Hong, S.; Farokhzad, O. C.; Margalit, R.; Langer, R., Nanocarriers as an emerging platform for cancer therapy. Nature nanotechnology 2007, 2 (12), 751-760.

37. Morral-Ruíz, G.; Melgar-Lesmes, P.; López-Vicente, A.; Solans, C.; García-Celma, M. J., Biotinylated polyurethane-urea nanoparticles for targeted theranostics in human hepatocellular carcinoma. Nano Research 2015, 8 (5), 1729-1745. 
38. Sabir, F.; Asad, M. I.; Qindeel, M.; Afzal, I.; Dar, M. J.; Shah, K. U.; Zeb, A.; Khan, G. M.; Ahmed, N.; Din, F.-u., Polymeric nanogels as versatile nanoplatforms for biomedical applications. Journal of nanomaterials 2019, 2019.

39. Soni, K. S.; Desale, S. S.; Bronich, T. K., Nanogels: An overview of properties, biomedical applications and obstacles to clinical translation. Journal of Controlled Release 2016, 240, 109-126.

40. Zhang, X.; Malhotra, S.; Molina, M.; Haag, R., Micro-and nanogels with labile crosslinks-from synthesis to biomedical applications. Chemical Society Reviews 2015, 44 (7), 1948-1973.

41. Li, Y.; Maciel, D.; Rodrigues, J.; Shi, X.; Tomas, H., Biodegradable polymer nanogels for drug/nucleic acid delivery. Chemical reviews 2015, 115 (16), 8564-8608.

42. Sivaram, A. J.; Rajitha, P.; Maya, S.; Jayakumar, R.; Sabitha, M., Nanogels for delivery, imaging and therapy. Wiley Interdisciplinary Reviews: Nanomedicine and Nanobiotechnology 2015, 7 (4), 509-533.

43. O'driscoll, C.; Griffin, B., Biopharmaceutical challenges associated with drugs with low aqueous solubility-the potential impact of lipid-based formulations. Advanced drug delivery reviews 2008, 60 (6), 617-624.

44. Tan, J. P.; Wang, Q.; Tam, K. C., Control of burst release from nanogels via layer by layer assembly. Journal of Controlled Release 2008, 128 (3), 248-254.

45. Astashkina, A.; Mann, B.; Grainger, D. W., A critical evaluation of in vitro cell culture models for high-throughput drug screening and toxicity. Pharmacology \& therapeutics 2012, 134 (1), 82-106.

46. $\quad \operatorname{van} \operatorname{der}$ Aa, M. A.; Mastrobattista, E.; Oosting, R. S.; Hennink, W. E.; Koning, G. A.; Crommelin, D. J., The nuclear pore complex: the gateway to successful nonviral gene delivery. Pharmaceutical research 2006, 23 (3), 447-459. 
47. Matsumura, Y.; Maeda, H., A new concept for macromolecular therapeutics in cancer chemotherapy: mechanism of tumoritropic accumulation of proteins and the antitumor agent smancs. Cancer research 1986, 46 (12 Part 1), 6387-6392.

48. Duncan, R., The dawning era of polymer therapeutics. Nature reviews Drug discovery 2003, 2 (5), 347-360.

49. Mammen, M.; Choi, S. K.; Whitesides, G. M., Polyvalent interactions in biological systems: implications for design and use of multivalent ligands and inhibitors. Angewandte Chemie International Edition 1998, 37 (20), 2754-2794.

50. Fasting, C.; Schalley, C. A.; Weber, M.; Seitz, O.; Hecht, S.; Koksch, B.; Dernedde, J.; Graf, C.; Knapp, E. W.; Haag, R., Multivalency as a chemical organization and action principle. Angewandte Chemie International Edition 2012, 51 (42), 1047210498.

51. Kabanov, A. V.; Vinogradov, S. V., Nanogels as pharmaceutical carriers: finite networks of infinite capabilities. Angewandte Chemie International Edition 2009, $48(30), 5418-5429$.

52. Laradji, A. M.; Kolesnikov, A. V.; Karakoçak, B. B.; Kefalov, V. J.; Ravi, N., RedoxResponsive Hyaluronic Acid-Based Nanogels for the Topical Delivery of the Visual Chromophore to Retinal Photoreceptors. ACS omega 2021, 6 (9), 6172-6184.

53. Mauri, E.; Moroni, I.; Magagnin, L.; Masi, M.; Sacchetti, A.; Rossi, F., Comparison between two different click strategies to synthesize fluorescent nanogels for therapeutic applications. Reactive and Functional Polymers 2016, 105, 35-44.

54. Chen, J.; Zou, Y.; Deng, C.; Meng, F.; Zhang, J.; Zhong, Z., Multifunctional click hyaluronic acid nanogels for targeted protein delivery and effective cancer treatment in vivo. Chemistry of Materials 2016, 28 (23), 8792-8799.

55. Asadi, H.; Khoee, S., Dual responsive nanogels for intracellular doxorubicin delivery. International journal of pharmaceutics 2016, 511 (1), 424-435. 
56. Noree, S.; Tangpasuthadol, V.; Kiatkamjornwong, S.; Hoven, V. P., Cascade postpolymerization modification of single pentafluorophenyl ester-bearing homopolymer as a facile route to redox-responsive nanogels. Journal of colloid and interface science 2017, 501, 94-102.

57. Harris, J. M.; Chess, R. B., Effect of pegylation on pharmaceuticals. Nature reviews Drug discovery 2003, 2 (3), 214-221.

58. Veronese, F. M.; Pasut, G., PEGylation, successful approach to drug delivery. Drug discovery today 2005, 10 (21), 1451-1458.

59. Mauri, E.; Veglianese, P.; Papa, S.; Mariani, A.; De Paola, M.; Rigamonti, R.; Chincarini, G. M.; Rimondo, S.; Sacchetti, A.; Rossi, F., Chemoselective functionalization of nanogels for microglia treatment. European Polymer Journal 2017, 94, 143-151.

60. Mauri, E.; Cappella, F.; Masi, M.; Rossi, F., PEGylation influences drug delivery from nanogels. Journal of Drug Delivery Science and Technology 2018, 46, 87-92.

61. Zhang, Z.; Wan, J.; Sun, L.; Li, Y.; Guo, J.; Wang, C., Zinc finger-inspired nanohydrogels with glutathione/pH triggered degradation based on coordination substitution for highly efficient delivery of anti-cancer drugs. Journal of Controlled Release 2016, 225, 96-108.

62. Antony, A. C., The biological chemistry of folate receptors. 1992.

63. Hilgenbrink, A. R.; Low, P. S., Folate receptor-mediated drug targeting: from therapeutics to diagnostics. Journal of pharmaceutical sciences 2005, 94 (10), 2135-2146.

64. Leamon, C. P.; Reddy, J. A., Folate-targeted chemotherapy. Advanced drug delivery reviews 2004, 56 (8), 1127-1141.

65. Low, P. S.; Henne, W. A.; Doorneweerd, D. D., Discovery and development of folicacid-based receptor targeting for imaging and therapy of cancer and inflammatory diseases. Accounts of chemical research 2008, 41 (1), 120-129. 
66. Lu, Y.; Low, P. S., Folate-mediated delivery of macromolecular anticancer therapeutic agents. Advanced drug delivery reviews 2012, 64, 342-352.

67. Sudimack, J.; Lee, R. J., Targeted drug delivery via the folate receptor. Advanced drug delivery reviews 2000, 41 (2), 147-162.

68. Massiah, M. A.; Matts, J. A.; Short, K. M.; Simmons, B. N.; Singireddy, S.; Yi, Z.; Cox, T. C., Solution structure of the MID1 B-box2 CHC (D/C) C2H2 zinc-binding domain: insights into an evolutionarily conserved RING fold. Journal of molecular biology 2007, 369 (1), 1-10.

69. Dietrich, R. A.; Richberg, M. H.; Schmidt, R.; Dean, C.; Dangl, J. L., A novel zinc finger protein is encoded by the Arabidopsis LSD1 gene and functions as a negative regulator of plant cell death. Cell 1997, 88 (5), 685-694.

70. Liu, S.; Huang, Y.; Chen, X.; Zhang, L.; Jing, X., Lactose mediated liver-targeting effect observed by ex vivo imaging technology. Biomaterials 2010, 31 (9), 26462654.

71. Lee, S.-Y.; Kim, S.; Tyler, J. Y.; Park, K.; Cheng, J.-X., Blood-stable, tumor-adaptable disulfide bonded mPEG-(Cys) 4-PDLLA micelles for chemotherapy. Biomaterials 2013, 34 (2), 552-561.

72. Zhu, Y.; Wang, X.; Chen, J.; Zhang, J.; Meng, F.; Deng, C.; Cheng, R.; Feijen, J.; Zhong, Z., Bioresponsive and fluorescent hyaluronic acid-iodixanol nanogels for targeted X-ray computed tomography imaging and chemotherapy of breast tumors. Journal of controlled release 2016, 244, 229-239.

73. Wang, Y.-C.; Wang, F.; Sun, T.-M.; Wang, J., Redox-responsive nanoparticles from the single disulfide bond-bridged block copolymer as drug carriers for overcoming multidrug resistance in cancer cells. Bioconjugate chemistry 2011, 22 (10), 19391945.

74. Sahay, G.; Alakhova, D. Y.; Kabanov, A. V., Endocytosis of nanomedicines. Journal of controlled release 2010, 145 (3), 182-195. 
75. Chen, Y.; Tezcan, O.; Li, D.; Beztsinna, N.; Lou, B.; Etrych, T.; Ulbrich, K.; Metselaar, J.; Lammers, T.; Hennink, W. E., Overcoming multidrug resistance using folate receptor-targeted and $\mathrm{pH}$-responsive polymeric nanogels containing covalently entrapped doxorubicin. Nanoscale 2017, 9 (29), 10404-10419.

76. Blenke, E. O.; Klaasse, G.; Merten, H.; Plückthun, A.; Mastrobattista, E.; Martin, N. I., Liposome functionalization with copper-free "click chemistry". Journal of Controlled Release 2015, 202, 14-20.

77. Lutz, J. F., Copper-free azide-alkyne cycloadditions: new insights and perspectives. Angewandte Chemie International Edition 2008, 47 (12), 2182-2184.

78. Jiang, Y.; Chen, J.; Deng, C.; Suuronen, E. J.; Zhong, Z., Click hydrogels, microgels and nanogels: Emerging platforms for drug delivery and tissue engineering. Biomaterials 2014, 35 (18), 4969-4985.

79. Lu, Y.; Low, P. S., Folate-mediated delivery of macromolecular anticancer therapeutic agents. Advanced Drug Delivery Reviews 2002, 54 (5), 675-693.

80. Aktan, B.; Chambre, L.; Sanyal, R.; Sanyal, A., “Clickable” Nanogels via Thermally Driven Self-Assembly of Polymers: Facile Access to Targeted Imaging Platforms using Thiol-Maleimide Conjugation. Biomacromolecules 2017, 18 (2), 490-497.

81. Li, L.; Raghupathi, K.; Song, C.; Prasad, P.; Thayumanavan, S., Self-assembly of random copolymers. Chemical communications 2014, 50 (88), 13417-13432.

82. Sipkins, D. A.; Cheresh, D. A.; Kazemi, M. R.; Nevin, L. M.; Bednarski, M. D.; Li, K. C., Detection of tumor angiogenesis in vivo by $\alpha \vee \beta 3$-targeted magnetic resonance imaging. Nature medicine 1998, 4 (5), 623-626.

83. Gao, X.; Yang, H.; Wu, M.; Shi, K.; Zhou, C.; Peng, J.; Yang, Q., Targeting delivery of lidocaine and cisplatin by nanogel enhances chemotherapy and alleviates metastasis. ACS applied materials \& interfaces 2018, 10 (30), 25228-25240. 
84. Chamaraux-Tran, T.-N.; Piegeler, T., The amide local anesthetic lidocaine in cancer surgery-potential antimetastatic effects and preservation of immune cell function? a narrative review. Frontiers in medicine 2017, 4, 235.

85. Li, K.; Yang, J.; Han, X., Lidocaine sensitizes the cytotoxicity of cisplatin in breast cancer cells via up-regulation of RAR $\beta 2$ and RASSF1A demethylation. International journal of molecular sciences 2014, 15 (12), 23519-23536.

86. Xing, W.; Chen, D.-T.; Pan, J.-H.; Chen, Y.-H.; Yan, Y.; Li, Q.; Xue, R.-F.; Yuan, Y.-F.; Zeng, W.-A., Lidocaine induces apoptosis and suppresses tumor growth in human hepatocellular carcinoma cells in vitro and in a xenograft model in vivo. Anesthesiology 2017, 126 (5), 868-881.

87. Duncan, R.; Gaspar, R., Nanomedicine (s) under the microscope. Molecular pharmaceutics 2011, 8 (6), 2101-2141.

88. Yang, X., lyer AK; Singh A.; Choy E.; Hornicek FJ; Amiji MM; Duan Z. Mdr1 Sirna Loaded Hyaluronic Acid-Based Cd44 Targeted Nanoparticle Systems Circumvent Paclitaxel Resistance in Ovarian Cancer. Sci. Rep 2015, 5 (8509.10), 1038.

89. Karakocak, B. B.; Liang, J.; Biswas, P.; Ravi, N., Hyaluronate coating enhances the delivery and biocompatibility of gold nanoparticles. Carbohydrate polymers 2018, $186,243-251$.

90. Schafer, F. Q.; Buettner, G. R., Redox environment of the cell as viewed through the redox state of the glutathione disulfide/glutathione couple. Free radical biology and medicine 2001, 30 (11), 1191-1212.

91. Khatun, Z.; Nurunnabi, M.; Nafiujjaman, M.; Reeck, G. R.; Khan, H. A.; Cho, K. J.; Lee, Y.-k., A hyaluronic acid nanogel for photo-chemo theranostics of lung cancer with simultaneous light-responsive controlled release of doxorubicin. Nanoscale 2015, 7 (24), 10680-10689.

92. Eslami, P.; Rossi, F.; Fedeli, S., Hybrid nanogels: stealth and biocompatible structures for drug delivery applications. Pharmaceutics 2019, 11 (2), 71. 
93. Liu, C.; Tai, L.; Zhang, W.; Wei, G.; Pan, W.; Lu, W., Penetratin, a potentially powerful absorption enhancer for noninvasive intraocular drug delivery. Molecular pharmaceutics 2014, 11 (4), 1218-1227.

94. Zhang, Q.; Colazo, J.; Berg, D.; Mugo, S. M.; Serpe, M. J., Multiresponsive nanogels for targeted anticancer drug delivery. Molecular pharmaceutics 2017, 14 (8), 2624-2628.

95. Zhu, Y.; Tong, W.; Gao, C., Molecular-engineered polymeric microcapsules assembled from Concanavalin $A$ and glycogen with specific responses to carbohydrates. Soft Matter 2011, 7 (12), 5805-5815.

96. Sato, K.; Kodama, D.; Anzai, J.-i., Electrochemical determination of sugars by use of multilayer thin films of ferrocene-appended glycogen and concanavalin A. Analytical and bioanalytical chemistry 2006, 386 (6), 1899-1904.

97. Dautry-Varsat, A.; Ciechanover, A.; Lodish, H. F., pH and the recycling of transferrin during receptor-mediated endocytosis. Proceedings of the National Academy of Sciences 1983, 80 (8), 2258-2262.

98. Ryschich, E.; Huszty, G.; Knaebel, H.; Hartel, M.; Büchler, M.; Schmidt, J., Transferrin receptor is a marker of malignant phenotype in human pancreatic cancer and in neuroendocrine carcinoma of the pancreas. European journal of cancer 2004, 40 (9), 1418-1422.

99. Li, D.; Sun, F.; Bourajjaj, M.; Chen, Y.; Pieters, E. H.; Chen, J.; Van Den Dikkenberg, J. B.; Lou, B.; Camps, M. G.; Ossendorp, F., Strong in vivo antitumor responses induced by an antigen immobilized in nanogels via reducible bonds. Nanoscale 2016, 8 (47), 19592-19604.

100. Palucka, K.; Banchereau, J., Dendritic-cell-based therapeutic cancer vaccines. Immunity 2013, 39 (1), 38-48. 
101. Nierkens, S.; Tel, J.; Janssen, E.; Adema, G. J., Antigen cross-presentation by dendritic cell subsets: one general or all sergeants? Trends in immunology 2013, 34 (8), 361-370.

102. Shima, F.; Uto, T.; Akagi, T.; Baba, M.; Akashi, M., Size effect of amphiphilic poly ( $\nu$-glutamic acid) nanoparticles on cellular uptake and maturation of dendritic cells in vivo. Acta biomaterialia 2013, 9 (11), 8894-8901.

103. Cheng, L.-C.; Jiang, X.; Wang, J.; Chen, C.; Liu, R.-S., Nano-bio effects: interaction of nanomaterials with cells. Nanoscale 2013, 5 (9), 3547-3569.

104. Blank, F.; Stumbles, P. A.; Seydoux, E.; Holt, P. G.; Fink, A.; Rothen-Rutishauser, B.; Strickland, D. H.; Von Garnier, C., Size-dependent uptake of particles by pulmonary antigen-presenting cell populations and trafficking to regional lymph nodes. American journal of respiratory cell and molecular biology 2013, 49 (1), 6777.

105. Jin, H.; Heller, D. A.; Sharma, R.; Strano, M. S., Size-dependent cellular uptake and expulsion of single-walled carbon nanotubes: single particle tracking and a generic uptake model for nanoparticles. ACS nano 2009, 3 (1), 149-158.

106. Ma, Y.; Zhuang, Y.; Xie, X.; Wang, C.; Wang, F.; Zhou, D.; Zeng, J.; Cai, L., The role of surface charge density in cationic liposome-promoted dendritic cell maturation and vaccine-induced immune responses. Nanoscale 2011, 3 (5), 2307-2314.

107. Adamo, G.; Grimaldi, N.; Sabatino, M. A.; Walo, M.; Dispenza, C.; Ghersi, G., Ebeam crosslinked nanogels conjugated with monoclonal antibodies in targeting strategies. Biological chemistry 2017, 398 (2), 277-287.

108. Lawrence, D. A.; Song, R.; Webert, P., Surface thiols of human lymphocytes and their changes after in vitro and in vivo activation. Journal of leukocyte biology 1996, 60 (5), 611-618.

109. Tang, L.; Zheng, Y.; Melo, M. B.; Mabardi, L.; Castaño, A. P.; Xie, Y.-Q.; Li, N.; Kudchodkar, S. B.; Wong, H. C.; Jeng, E. K., Enhancing T cell therapy through TCR- 
signaling-responsive nanoparticle drug delivery. Nature biotechnology 2018, 36 (8), 707-716.

110. Klebanoff, C. A.; Finkelstein, S. E.; Surman, D. R.; Lichtman, M. K.; Gattinoni, L.; Theoret, M. R.; Grewal, N.; Spiess, P. J.; Antony, P. A.; Palmer, D. C., IL-15 enhances the in vivo antitumor activity of tumor-reactive CD8+ T cells. Proceedings of the National Academy of Sciences 2004, 101 (7), 1969-1974.

111. Zhu, X.; Marcus, W. D.; Xu, W.; Lee, H.-i.; Han, K.; Egan, J. O.; Yovandich, J. L.; Rhode, P. R.; Wong, H. C., Novel human interleukin-15 agonists. The Journal of Immunology 2009, 183 (6), 3598-3607.

112. Rhode, P. R.; Egan, J. O.; Xu, W.; Hong, H.; Webb, G. M.; Chen, X.; Liu, B.; Zhu, X.; Wen, J.; You, L., Comparison of the superagonist complex, ALT-803, to IL15 as cancer immunotherapeutics in animal models. Cancer immunology research 2016, 4 (1), 49-60.

113. Gonçalves, C.; Ferreira, S. A.; Correia, A. L.; Lopes, C.; Fleming, C. E.; Rocha, E.; Vilanova, M.; Gama, M., Potential of mannan or dextrin nanogels as vaccine carrier/adjuvant systems. Journal of Bioactive and Compatible Polymers 2016, 31 (5), 453-466.

114. De Temmerman, M.-L.; Rejman, J.; Demeester, J.; Irvine, D. J.; Gander, B.; De Smedt, S. C., Particulate vaccines: on the quest for optimal delivery and immune response. Drug discovery today 2011, 16 (13-14), 569-582.

115. Wang, X.; Luo, J.; He, L.; Cheng, X.; Yan, G.; Wang, J.; Tang, R., Hybrid pH-sensitive nanogels surface-functionalized with collagenase for enhanced tumor penetration. Journal of colloid and interface science 2018, 525, 269-281.

116. Yang, G.; Wang, X.; Fu, S.; Tang, R.; Wang, J., pH-triggered chitosan nanogels via an ortho ester-based linkage for efficient chemotherapy. Acta biomaterialia 2017, $60,232-243$. 
117. Nishimura, T.; Yamada, A.; Umezaki, K.; Sawada, S.-i.; Mukai, S.-a.; Sasaki, Y.; Akiyoshi, K., Self-assembled polypeptide nanogels with enzymatically transformable surface as a small interfering RNA delivery platform. Biomacromolecules 2017, 18 (12), 3913-3923.

118. Thelu, H. V. P.; Atchimnaidu, S.; Perumal, D.; Harikrishnan, K. S.; Vijayan, S.; Varghese, R., Self-assembly of an aptamer-decorated, DNA-protein hybrid nanogel: a biocompatible nanocarrier for targeted cancer therapy. ACS Applied Bio Materials 2019, 2 (12), 5227-5234.

119. Lou, S.; Gao, S.; Wang, W.; Zhang, M.; Zhang, J.; Wang, C.; Li, C.; Kong, D.; Zhao, Q., Galactose-functionalized multi-responsive nanogels for hepatoma-targeted drug delivery. Nanoscale 2015, 7 (7), 3137-3146.

120. Salehi, R.; Rasouli, S.; Hamishehkar, H., Smart thermo/pH responsive magnetic nanogels for the simultaneous delivery of doxorubicin and methotrexate. International journal of pharmaceutics 2015, 487 (1-2), 274-284.

121. Peng, J.; Qi, T.; Liao, J.; Chu, B.; Yang, Q.; Li, W.; Qu, Y.; Luo, F.; Qian, Z., Controlled release of cisplatin from $\mathrm{pH}$-thermal dual responsive nanogels. Biomaterials 2013, 34 (34), 8726-8740.

122. Klaikherd, A.; Nagamani, C.; Thayumanavan, S., Multi-stimuli sensitive amphiphilic block copolymer assemblies. Journal of the American Chemical Society 2009, 131 (13), 4830-4838.

123. Schuller, V. J.; Heidegger, S.; Sandholzer, N.; Nickels, P. C.; Suhartha, N. A.; Endres, S.; Bourquin, C.; Liedl, T., Cellular immunostimulation by CpG-sequence-coated DNA origami structures. ACS nano 2011, 5 (12), 9696-9702.

124. Cao, Y.; Hu, X.-Y.; Li, Y.; Zou, X.; Xiong, S.; Lin, C.; Shen, Y.-Z.; Wang, L., Multistimuliresponsive supramolecular vesicles based on water-soluble pillar [6] arene and SAINT complexation for controllable drug release. Journal of the American Chemical Society 2014, 136 (30), 10762-10769. 
125. Zhang, Q.; Jiang, Q.; Li, N.; Dai, L.; Liu, Q.; Song, L.; Wang, J.; Li, Y.; Tian, J.; Ding, B., DNA origami as an in vivo drug delivery vehicle for cancer therapy. ACS nano 2014, 8 (7), 6633-6643.

126. Zhou, X.; Su, X.; Pathak, P.; Vik, R.; Vinciguerra, B.; Isaacs, L.; Jayawickramarajah, J., Host-guest tethered DNA transducer: ATP fueled release of a protein inhibitor from Cucurbit [7] uril. Journal of the American Chemical Society 2017, 139 (39), 13916-13921.

127. Ramya, A. N.; Joseph, M. M.; Maniganda, S.; Karunakaran, V.; TT, S.; Maiti, K. K., Emergence of gold-mesoporous silica hybrid nanotheranostics: dox-encoded, folate targeted chemotherapy with modulation of SERS fingerprinting for apoptosis toward tumor eradication. Small 2017, 13 (31), 1700819.

128. Lu, Y.; Yue, Z.; Xie, J.; Wang, W.; Zhu, H.; Zhang, E.; Cao, Z., Micelles with ultralow critical micelle concentration as carriers for drug delivery. Nature biomedical engineering 2018, 2 (5), 318-325.

129. Li, N.; Xiang, M.-H.; Liu, J.-W.; Tang, H.; Jiang, J.-H., DNA polymer nanoparticles programmed via supersandwich hybridization for imaging and therapy of cancer cells. Analytical chemistry 2018, 90 (21), 12951-12958.

130. Sujai, P. T.; Joseph, M. M.; Karunakaran, V.; Saranya, G.; Adukkadan, R. N.; Shamjith, S.; Thomas, R.; Nair, J. B.; Swathi, R. S.; Maiti, K. K., Biogenic clusterencased gold nanorods as a targeted three-in-one theranostic nanoenvelope for SERS-guided photochemotherapy against metastatic melanoma. ACS Applied Bio Materials 2018, 2 (1), 588-600.

131. Zhou, T.; Li, J.; Jia, X.; Zhao, X.; Liu, P., pH/Reduction dual-responsive oxidized alginate-doxorubicin (mPEG-OAL-DOX/Cys) prodrug nanohydrogels: Effect of complexation with cyclodextrins. Langmuir 2018, 34 (1), 416-424.

132. Li, Z.; Zheng, Z.; Su, S.; Yu, L.; Wang, X., Hydroxypropyl- $\beta$-CD vs. its $\alpha$-homologue for a 3D modified polyrotaxane network formation and properties: the 
relationship between modified $C D$ and polymer revealed through comparison. Soft matter 2016, 12 (34), 7089-7101.

133. Wang, H.; Dai, T.; Zhou, S.; Huang, X.; Li, S.; Sun, K.; Zhou, G.; Dou, H., Selfassembly assisted fabrication of dextran-based nanohydrogels with reductioncleavable junctions for applications as efficient drug delivery systems. Scientific reports 2017, 7 (1), 1-12.

134. Li, Y.; Wang, J.; Wientjes, M. G.; Au, J. L.-S., Delivery of nanomedicines to extracellular and intracellular compartments of a solid tumor. Advanced drug delivery reviews 2012, 64 (1), 29-39.

135. Danhier, F.; Feron, O.; Préat, V., To exploit the tumor microenvironment: passive and active tumor targeting of nanocarriers for anti-cancer drug delivery. Journal of controlled release 2010, 148 (2), 135-146.

136. Kuppusamy, P.; Afeworki, M.; Shankar, R. A.; Coffin, D.; Krishna, M. C.; Hahn, S. M.; Mitchell, J. B.; Zweier, J. L., In vivo electron paramagnetic resonance imaging of tumor heterogeneity and oxygenation in a murine model. Cancer Research 1998, 58 (7), 1562-1568.

137. Kuppusamy, P.; Li, H.; Ilangovan, G.; Cardounel, A. J.; Zweier, J. L.; Yamada, K.; Krishna, M. C.; Mitchell, J. B., Noninvasive imaging of tumor redox status and its modification by tissue glutathione levels. Cancer research 2002, 62 (1), 307-312.

138. Gao, C.; Lin, Z.; Jurado-Sánchez, B.; Lin, X.; Wu, Z.; He, Q., Stem cell membranecoated nanogels for highly efficient in vivo tumor targeted drug delivery. Small 2016, 12 (30), 4056-4062.

139. Uccelli, A.; Moretta, L.; Pistoia, V., Mesenchymal stem cells in health and disease. Nature reviews immunology 2008, 8 (9), 726-736.

140. Studeny, M.; Marini, F. C.; Dembinski, J. L.; Zompetta, C.; Cabreira-Hansen, M.; Bekele, B. N.; Champlin, R. E.; Andreeff, M., Mesenchymal stem cells: potential 
precursors for tumor stroma and targeted-delivery vehicles for anticancer agents. Journal of the National Cancer Institute 2004, 96 (21), 1593-1603.

141. Yu, L.; Dong, A.; Guo, R.; Yang, M.; Deng, L.; Zhang, J., DOX/ICG coencapsulated liposome-coated thermosensitive nanogels for NIR-triggered simultaneous drug release and photothermal effect. ACS Biomaterials Science \& Engineering 2018, 4 (7), 2424-2434.

142. Shanmugam, V.; Selvakumar, S.; Yeh, C.-S., Near-infrared light-responsive nanomaterials in cancer therapeutics. Chemical Society Reviews 2014, 43 (17), 6254-6287.

143. Zhang, Z.; Wang, J.; Chen, C., Near-infrared light-mediated nanoplatforms for cancer thermo-chemotherapy and optical imaging. Advanced Materials 2013, 25 (28), 3869-3880.

144. Zou, L.; Wang, H.; He, B.; Zeng, L.; Tan, T.; Cao, H.; He, X.; Zhang, Z.; Guo, S.; Li, Y., Current approaches of photothermal therapy in treating cancer metastasis with nanotherapeutics. Theranostics 2016, 6 (6), 762.

145. Raemdonck, K.; Braeckmans, K.; Demeester, J.; De Smedt, S. C., Merging the best of both worlds: hybrid lipid-enveloped matrix nanocomposites in drug delivery. Chemical Society Reviews 2014, 43 (1), 444-472.

146. Kazakov, S., Liposome-nanogel structures for future pharmaceutical applications: an updated review. Current pharmaceutical design 2016, 22 (10), 1391-1413.

147. Mandal, B.; Bhattacharjee, H.; Mittal, N.; Sah, H.; Balabathula, P.; Thoma, L. A.; Wood, G. C., Core-shell-type lipid-polymer hybrid nanoparticles as a drug delivery platform. Nanomedicine: Nanotechnology, Biology and Medicine 2013, 9 (4), 474-491.

148. Chen, J.; Ding, J.; Xu, W.; Sun, T.; Xiao, H.; Zhuang, X.; Chen, X., Receptor and microenvironment dual-recognizable nanogel for targeted chemotherapy of highly metastatic malignancy. Nano letters 2017, 17 (7), 4526-4533. 
149. Deshayes, S.; Cabral, H.; Ishii, T.; Miura, Y.; Kobayashi, S.; Yamashita, T.; Matsumoto, A.; Miyahara, Y.; Nishiyama, N.; Kataoka, K., Phenylboronic acidinstalled polymeric micelles for targeting sialylated epitopes in solid tumors. Journal of the American Chemical Society 2013, 135 (41), 15501-15507.

150. Guo, X.; Wei, X.; Jing, Y.; Zhou, S., Size changeable nanocarriers with nuclear targeting for effectively overcoming multidrug resistance in cancer therapy. Advanced materials 2015, 27 (41), 6450-6456.

151. Zhang, Y.; Wang, C.; Xu, C.; Yang, C.; Zhang, Z.; Yan, H.; Liu, K., Morpholinodecorated long circulating polymeric micelles with the function of surface charge transition triggered by $\mathrm{pH}$ changes. Chemical Communications 2013, 49 (66), 7286-7288.

152. Chen, J.; Ding, J.; Xiao, C.; Zhuang, X.; Chen, X., Emerging antitumor applications of extracellularly reengineered polymeric nanocarriers. Biomaterials science 2015, 3 (7), 988-1001.

153. Torre, L. A.; Bray, F.; Siegel, R. L.; Ferlay, J.; Lortet-Tieulent, J.; Jemal, A., Global cancer statistics, 2012. CA: a cancer journal for clinicians 2015, 65 (2), 87-108.

154. Sadok, A.; McCarthy, A.; Caldwell, J.; Collins, I.; Garrett, M. D.; Yeo, M.; Hooper, S.; Sahai, E.; Kuemper, S.; Mardakheh, F. K., Rho kinase inhibitors block melanoma cell migration and inhibit metastasis. Cancer research 2015, 75 (11), 2272-2284.

155. Friedl, P.; Wolf, K., Tumour-cell invasion and migration: diversity and escape mechanisms. Nature reviews cancer 2003, 3 (5), 362-374.

156. Rao, N. V.; Yoon, H. Y.; Han, H. S.; Ko, H.; Son, S.; Lee, M.; Lee, H.; Jo, D.-G.; Kang, Y. M.; Park, J. H., Recent developments in hyaluronic acid-based nanomedicine for targeted cancer treatment. Expert opinion on drug delivery 2016, 13 (2), 239-252.

157. Oh, E. J.; Park, K.; Kim, K. S.; Kim, J.; Yang, J.-A.; Kong, J.-H.; Lee, M. Y.; Hoffman, A. S.; Hahn, S. K., Target specific and long-acting delivery of protein, peptide, and 
nucleotide therapeutics using hyaluronic acid derivatives. Journal of Controlled Release 2010, 141 (1), 2-12.

158. Zhong, Y.; Zhang, J.; Cheng, R.; Deng, C.; Meng, F.; Xie, F.; Zhong, Z., Reversibly crosslinked hyaluronic acid nanoparticles for active targeting and intelligent delivery of doxorubicin to drug resistant CD44+ human breast tumor xenografts. Journal of controlled release 2015, 205, 144-154.

159. Lim, R. K.; Lin, Q., Photoinducible bioorthogonal chemistry: a spatiotemporally controllable tool to visualize and perturb proteins in live cells. Accounts of chemical research 2011, 44 (9), 828-839.

160. Murakami, M.; Cabral, H.; Matsumoto, Y.; Wu, S.; Kano, M. R.; Yamori, T.; Nishiyama, N.; Kataoka, K., Improving drug potency and efficacy by nanocarriermediated subcellular targeting. Science translational medicine 2011, 3 (64), 64ra2-64ra2.

161. Hrkach, J.; Von Hoff, D.; Ali, M. M.; Andrianova, E.; Auer, J.; Campbell, T.; De Witt, D.; Figa, M.; Figueiredo, M.; Horhota, A., Preclinical development and clinical translation of a PSMA-targeted docetaxel nanoparticle with a differentiated pharmacological profile. Science translational medicine 2012, 4 (128), 128ra39128 ra39.

162. Hu, Q.; Rijcken, C. J.; Bansal, R.; Hennink, W. E.; Storm, G.; Prakash, J., Complete regression of breast tumour with a single dose of docetaxel-entrapped core-crosslinked polymeric micelles. Biomaterials 2015, 53, 370-378.

163. Callmann, C. E.; Barback, C. V.; Thompson, M. P.; Hall, D. J.; Mattrey, R. F.; Gianneschi, N. C., Therapeutic enzyme-responsive nanoparticles for targeted delivery and accumulation in tumors. Advanced materials 2015, 27 (31), 46114615 .

164. Li, Q.-L.; Sun, Y.; Sun, Y.-L.; Wen, J.; Zhou, Y.; Bing, Q.-M.; Isaacs, L. D.; Jin, Y.; Gao, H.; Yang, Y.-W., Mesoporous silica nanoparticles coated by layer-by-layer self- 
assembly using cucurbit [7] uril for in vitro and in vivo anticancer drug release. Chemistry of Materials 2014, 26 (22), 6418-6431.

165. Yu, J.; Zhang, Y.; Sun, W.; Wang, C.; Ranson, D.; Ye, Y.; Weng, Y.; Gu, Z., Internalized compartments encapsulated nanogels for targeted drug delivery. Nanoscale 2016, 8 (17), 9178-9184.

166. Li, H.; Guo, L.; Li, J.-W.; Liu, N.; Qi, R.; Liu, J., Expression of hyaluronan receptors CD44 and RHAMM in stomach cancers: relevance with tumor progression. International journal of oncology 2000, 17 (5), 927-959.

167. Gurski, L. A.; Jha, A. K.; Zhang, C.; Jia, X.; Farach-Carson, M. C., Hyaluronic acidbased hydrogels as 3D matrices for in vitro evaluation of chemotherapeutic drugs using poorly adherent prostate cancer cells. Biomaterials 2009, 30 (30), 60766085.

168. Greiner, J.; Schmitt, A.; Giannopoulos, K.; Rojewski, M. T.; Götz, M.; Funk, I.; Ringhoffer, M.; Bunjes, D.; Hofmann, S.; Ritter, G., High-dose RHAMM-R3 peptide vaccination for patients with acute myeloid leukemia, myelodysplastic syndrome and multiple myeloma. Haematologica 2010, 95 (7), 1191.

169. Hamilton, S. R.; Fard, S. F.; Paiwand, F. F.; Tolg, C.; Veiseh, M.; Wang, C.; McCarthy, J. B.; Bissell, M. J.; Koropatnick, J.; Turley, E. A., The hyaluronan receptors CD44 and Rhamm (CD168) form complexes with ERK1, 2 that sustain high basal motility in breast cancer cells. Journal of Biological Chemistry 2007, 282 (22), 1666716680.

170. Augustin, F.; Fiegl, M.; Schmid, T.; Pomme, G.; Sterlacci, W.; Tzankov, A., Receptor for hyaluronic acid-mediated motility (RHAMM, CD168) expression is prognostically important in both nodal negative and nodal positive large cell lung cancer. Journal of clinical pathology 2015, 68 (5), 368-373.

171. Gibbs, P.; Brown, T. J.; Ng, R.; Jennens, R.; Cinc, E.; Pho, M.; Michael, M.; Fox, R. M., A pilot human evaluation of a formulation of irinotecan and hyaluronic acid in 
5-fluorouracil-refractory metastatic colorectal cancer patients. Chemotherapy 2009, 55 (1), 49-59.

172. Misra, S.; Hascall, V. C.; Markwald, R. R.; Ghatak, S., Interactions between hyaluronan and its receptors (CD44, RHAMM) regulate the activities of inflammation and cancer. Frontiers in immunology 2015, 6, 201.

173. Yang, C.; Li, C.; Zhang, P.; Wu, W.; Jiang, X., Redox responsive hyaluronic acid nanogels for treating RHAMM (CD168) over-expressive cancer, both primary and metastatic tumors. Theranostics 2017, 7 (6), 1719.

174. Pikabea, A.; Villar-Álvarez, E.; Forcada, J.; Taboada, P., pH-controlled doxorubicin delivery from PDEAEMA-based nanogels. Journal of Molecular Liquids 2018, 266, 321-329.

175. Hang, C.; Zou, Y.; Zhong, Y.; Zhong, Z.; Meng, F., NIR and UV-responsive degradable hyaluronic acid nanogels for CD44-targeted and remotely triggered intracellular doxorubicin delivery. Colloids and Surfaces B: Biointerfaces 2017, 158, 547-555.

176. Zhao, X.; Deng, L.; Deng, H.; Dong, A.; Wang, W.; Zhang, J., In Situ Template Polymerization to Prepare Liposome-Coated PDMAEMA Nanogels with Controlled Size, High Stability, Low Cytotoxicity, and Responsive Drug Release for Intracellular DOX Release. Macromolecular Chemistry and Physics 2018, 219 (13), 1800071.

177. Carafa, M.; Marianecci, C.; Di Marzio, L.; Rinaldi, F.; Di Meo, C.; Matricardi, P.; Alhaique, F.; Coviello, T., A new vesicle-loaded hydrogel system suitable for topical applications: preparation and characterization. Journal of Pharmacy \& Pharmaceutical Sciences 2011, 14 (3), 336-346.

178. Mavuso, S.; Marimuthu, T.; E Choonara, Y.; Kumar, P.; C du Toit, L.; Pillay, V., A review of polymeric colloidal nanogels in transdermal drug delivery. Current pharmaceutical design 2015, 21 (20), 2801-2813. 
179. Rancan, F.; Asadian-Birjand, M.; Dogan, S.; Graf, C.; Cuellar, L.; Lommatzsch, S.; Blume-Peytavi, U.; Calderón, M.; Vogt, A., Effects of thermoresponsivity and softness on skin penetration and cellular uptake of polyglycerol-based nanogels. Journal of Controlled Release 2016, 228, 159-169.

180. Asadian-Birjand, M.; Bergueiro, J.; Rancan, F.; Cuggino, J. C.; Mutihac, R.-C.; Achazi, K.; Dernedde, J.; Blume-Peytayi, U.; Vogt, A.; Calderón, M., Engineering thermoresponsive polyether-based nanogels for temperature dependent skin penetration. Polymer Chemistry 2015, 6 (32), 5827-5831.

181. Witting, M.; Molina, M.; Obst, K.; Plank, R.; Eckl, K. M.; Hennies, H. C.; Calderón, M.; Frieß, W.; Hedtrich, S., Thermosensitive dendritic polyglycerol-based nanogels for cutaneous delivery of biomacromolecules. Nanomedicine: Nanotechnology, Biology and Medicine 2015, 11 (5), 1179-1187.

182. Edlich, A.; Gerecke, C.; Giulbudagian, M.; Neumann, F.; Hedtrich, S.; SchäferKorting, M.; Ma, N.; Calderon, M.; Kleuser, B., Specific uptake mechanisms of welltolerated thermoresponsive polyglycerol-based nanogels in antigen-presenting cells of the skin. European Journal of Pharmaceutics and Biopharmaceutics 2017, $116,155-163$.

183. Gerecke, C.; Edlich, A.; Giulbudagian, M.; Schumacher, F.; Zhang, N.; Said, A.; Yealland, G.; Lohan, S. B.; Neumann, F.; Meinke, M. C., Biocompatibility and characterization of polyglycerol-based thermoresponsive nanogels designed as novel drug-delivery systems and their intracellular localization in keratinocytes. Nanotoxicology 2017, 11 (2), 267-277.

184. Cuggino, J. C.; Strumia, M. C.; Igarzabal, C. I. A., Synthesis, characterization and slow drug delivery of hydrogels based in $\mathrm{N}$-acryloyl-tris-(hydroxymethyl) aminomethane and $\mathrm{N}$-isopropyl acrylamide. Reactive and Functional Polymers 2011, 71 (4), 440-446. 
185. Nel, A.; Xia, T.; Mädler, L.; Li, N., Toxic potential of materials at the nanolevel. science 2006, 311 (5761), 622-627.

186. Eom, H.-J.; Choi, J., p38 MAPK activation, DNA damage, cell cycle arrest and apoptosis as mechanisms of toxicity of silver nanoparticles in Jurkat T cells. Environmental science \& technology 2010, 44 (21), 8337-8342.

187. Hackenberg, S.; Scherzed, A.; Kessler, M.; Hummel, S.; Technau, A.; Froelich, K.; Ginzkey, C.; Koehler, C.; Hagen, R.; Kleinsasser, N., Silver nanoparticles: evaluation of DNA damage, toxicity and functional impairment in human mesenchymal stem cells. Toxicology letters 2011, 201 (1), 27-33.

188. Zhou, X.; Lin, A.; Yuan, X.; Li, H.; Ma, D.; Xue, W., Glucose-sensitive and bloodcompatible nanogels for insulin controlled release. Journal of Applied Polymer Science 2016, 133 (24).

189. Zhang, M.-J.; Wang, W.; Xie, R.; Ju, X.-J.; Liu, L.; Gu, Y.-Y.; Chu, L.-Y., Microfluidic fabrication of monodisperse microcapsules for glucose-response at physiological temperature. Soft Matter 2013, 9 (16), 4150-4159.

190. Lee, B. R.; Oh, K. T.; Oh, Y. T.; Baik, H. J.; Park, S. Y.; Youn, Y. S.; Lee, E. S., A novel pH-responsive polysaccharidic ionic complex for proapoptotic D-(KLAKLAK) 2 peptide delivery. Chemical communications 2011, 47 (13), 3852-3854.

191. Mai, J. C.; Mi, Z.; Kim, S.-H.; Ng, B.; Robbins, P. D., A proapoptotic peptide for the treatment of solid tumors. Cancer research 2001, 61 (21), 7709-7712.

192. Kim, S. K.; Youn, Y. S.; Oh, K. T.; Lee, E. S., Development of pH-responsive starchglycol chitosan nanogels for proapoptotic (KLAKLAK) 2 peptide delivery. Journal of Bioactive and Compatible Polymers 2017, 32 (4), 345-354.

193. Luo, D.; Saltzman, W. M., Synthetic DNA delivery systems. Nature biotechnology 2000, 18 (1), 33-37.

194. Dimde, M.; Neumann, F.; Reisbeck, F.; Ehrmann, S.; Cuellar-Camacho, J. L.; Steinhilber, D.; Ma, N.; Haag, R., Defined pH-sensitive nanogels as gene delivery 
platform for siRNA mediated in vitro gene silencing. Biomaterials science 2017, 5 (11), 2328-2336.

195. Park, J. S.; Yi, S. W.; Kim, H. J.; Kim, S. M.; Shim, S. H.; Park, K.-H., Sunflower-type nanogels carrying a quantum dot nanoprobe for both superior gene delivery efficacy and tracing of human mesenchymal stem cells. Biomaterials 2016, 77, 1425.

196. Park, J. S.; Yi, S. W.; Kim, H. J.; Park, K.-H., Receptor-mediated gene delivery into human mesenchymal stem cells using hyaluronic acid-shielded polyethylenimine/pDNA nanogels. Carbohydrate polymers 2016, 136, 791-802.

197. Shao, X. R.; Wei, X. Q.; Song, X.; Hao, L. Y.; Cai, X. X.; Zhang, Z. R.; Peng, Q.; Lin, Y. F., Independent effect of polymeric nanoparticle zeta potential/surface charge, on their cytotoxicity and affinity to cells. Cell proliferation 2015, 48 (4), 465-474.

198. Li, R.-Q.; Wu, W.; Song, H.-Q.; Ren, Y.; Yang, M.; Li, J.; Xu, F.-J., Well-defined reducible cationic nanogels based on functionalized low-molecular-weight PGMA for effective pDNA and siRNA delivery. Acta biomaterialia 2016, 41, 282-292.

199. Lai, M.-C.; Yang, Z.; Zhou, L.; Zhu, Q.-q.; Xie, H.-y.; Zhang, F.; Wu, L.-m.; Chen, L.m.; Zheng, S.-s., Long non-coding RNA MALAT-1 overexpression predicts tumor recurrence of hepatocellular carcinoma after liver transplantation. Medical oncology 2012, 29 (3), 1810-1816.

200. Leader, B.; Baca, Q. J.; Golan, D. E., Protein therapeutics: a summary and pharmacological classification. Nature reviews Drug discovery 2008, 7 (1), 21-39.

201. Zeng, Z.; She, Y.; Peng, Z.; Wei, J.; He, X., Enzyme-mediated in situ formation of pH-sensitive nanogels for proteins delivery. RSC advances 2016, 6 (10), 80328042.

202. Biswas, A.; Joo, K.-I.; Liu, J.; Zhao, M.; Fan, G.; Wang, P.; Gu, Z.; Tang, Y., Endoprotease-mediated intracellular protein delivery using nanocapsules. ACS nano 2011, 5 (2), 1385-1394. 
203. Chen, W.; Zheng, M.; Meng, F.; Cheng, R.; Deng, C.; Feijen, J.; Zhong, Z., In situ forming reduction-sensitive degradable nanogels for facile loading and triggered intracellular release of proteins. Biomacromolecules 2013, 14 (4), 1214-1222.

204. Sun, H.; Meng, F.; Cheng, R.; Deng, C.; Zhong, Z., Reduction and pH dualbioresponsive crosslinked polymersomes for efficient intracellular delivery of proteins and potent induction of cancer cell apoptosis. Acta biomaterialia 2014, 10 (5), 2159-2168.

205. Choi, J. H.; Jang, J. Y.; Joung, Y. K.; Kwon, M. H.; Park, K. D., Intracellular delivery and anti-cancer effect of self-assembled heparin-Pluronic nanogels with RNase A. Journal of controlled release 2010, 147 (3), 420-427.

206. Wang, M.; Alberti, K.; Sun, S.; Arellano, C. L.; Xu, Q., Combinatorially designed lipid-like nanoparticles for intracellular delivery of cytotoxic protein for cancer therapy. Angewandte Chemie International Edition 2014, 53 (11), 2893-2898.

207. Zhao, M.; Liu, Y.; Hsieh, R. S.; Wang, N.; Tai, W.; Joo, K.-I.; Wang, P.; Gu, Z.; Tang, Y., Clickable protein nanocapsules for targeted delivery of recombinant p53 protein. Journal of the American chemical society 2014, 136 (43), 15319-15325.

208. Liang, K.; Ng, S.; Lee, F.; Lim, J.; Chung, J. E.; Lee, S. S.; Kurisawa, M., Targeted intracellular protein delivery based on hyaluronic acid-green tea catechin nanogels. Acta biomaterialia 2016, 33, 142-152.

209. Tachibana, H.; Koga, K.; Fujimura, Y.; Yamada, K., A receptor for green tea polyphenol EGCG. Nature structural \& molecular biology 2004, 11 (4), 380-381.

210. Yang, C. S.; Lambert, J. D.; Sang, S., Antioxidative and anti-carcinogenic activities of tea polyphenols. Archives of toxicology 2009, 83 (1), 11-21.

211. Maiti, T. K.; Ghosh, K. S.; Dasgupta, S., Interaction of (-)-epigallocatechin-3-gallate with human serum albumin: Fluorescence, fourier transform infrared, circular dichroism, and docking studies. Proteins: Structure, Function, and Bioinformatics 2006, 64 (2), 355-362. 
212. Chung, J. E.; Tan, S.; Gao, S. J.; Yongvongsoontorn, N.; Kim, S. H.; Lee, J. H.; Choi, H. S.; Yano, H.; Zhuo, L.; Kurisawa, M., Self-assembled micellar nanocomplexes comprising green tea catechin derivatives and protein drugs for cancer therapy. Nature nanotechnology 2014, 9 (11), 907-912.

213. Lakhani, S. A.; Masud, A.; Kuida, K.; Porter, G. A.; Booth, C. J.; Mehal, W. Z.; Inayat, I.; Flavell, R. A., Caspases 3 and 7: key mediators of mitochondrial events of apoptosis. Science 2006, 311 (5762), 847-851.

214. Alves, N.; Leonor, I.; Azevedo, H. S.; Reis, R.; Mano, J., Designing biomaterials based on biomineralization of bone. Journal of Materials Chemistry 2010, 20 (15), 2911-2921.

215. Ma, J.; Wang, J.; Ai, X.; Zhang, S., Biomimetic self-assembly of apatite hybrid materials: from a single molecular template to bi-/multi-molecular templates. Biotechnology advances 2014, 32 (4), 744-760.

216. Tada, S.; Chowdhury, E. H.; Cho, C.-S.; Akaike, T., pH-sensitive carbonate apatite as an intracellular protein transporter. Biomaterials 2010, 31 (6), 1453-1459.

217. Qin, J.; Zhong, Z.; Ma, J., Biomimetic synthesis of hybrid hydroxyapatite nanoparticles using nanogel template for controlled release of bovine serum albumin. Materials Science and Engineering: C 2016, 62, 377-383.

218. Venkatesan, J.; Kim, S.-K., Chitosan composites for bone tissue engineering-an overview. Marine drugs 2010, 8 (8), 2252-2266.

219. Wang, C.; Li, P.; Liu, L.; Pan, H.; Li, H.; Cai, L.; Ma, Y., Self-adjuvanted nanovaccine for cancer immunotherapy: Role of lysosomal rupture-induced ROS in MHC class I antigen presentation. Biomaterials 2016, 79, 88-100.

220. Nuhn, L.; Vanparijs, N.; De Beuckelaer, A.; Lybaert, L.; Verstraete, G.; Deswarte, K.; Lienenklaus, S.; Shukla, N. M.; Salyer, A. C.; Lambrecht, B. N., pH-degradable imidazoquinoline-ligated nanogels for lymph node-focused immune activation. Proceedings of the National Academy of Sciences 2016, 113 (29), 8098-8103. 
221. Dudek, A. Z.; Yunis, C.; Harrison, L. I.; Kumar, S.; Hawkinson, R.; Cooley, S.; Vasilakos, J. P.; Gorski, K. S.; Miller, J. S., First in human phase I trial of 852A, a novel systemic toll-like receptor 7 agonist, to activate innate immune responses in patients with advanced cancer. Clinical Cancer Research 2007, 13 (23), 71197125.

222. Weigel, B. J.; Cooley, S.; DeFor, T.; Weisdorf, D. J.; Panoskaltsis-Mortari, A.; Chen, W.; Blazar, B. R.; Miller, J. S., Prolonged subcutaneous administration of 852A, a novel systemic toll-like receptor 7 agonist, to activate innate immune responses in patients with advanced hematologic malignancies. American journal of hematology 2012, 87 (10), 953-956.

223. Eberhardt, M.; Mruk, R.; Zentel, R.; Théato, P., Synthesis of pentafluorophenyl (meth) acrylate polymers: New precursor polymers for the synthesis of multifunctional materials. European Polymer Journal 2005, 41 (7), 1569-1575.

224. Narayan, R.; Nguyen, H.; Bentow, J. J.; Moy, L.; Lee, D. K.; Greger, S.; Haskell, J.; Vanchinathan, V.; Chang, P.-L.; Tsui, S., Immunomodulation by imiquimod in patients with high-risk primary melanoma. Journal of Investigative Dermatology 2012, 132 (1), 163-169.

225. Hemmi, H.; Kaisho, T.; Takeuchi, O.; Sato, S.; Sanjo, H.; Hoshino, K.; Horiuchi, T.; Tomizawa, H.; Takeda, K.; Akira, S., Small anti-viral compounds activate immune cells via the TLR7 MyD88-dependent signaling pathway. Nature immunology 2002, 3 (2), 196-200. 
Chapter 2

98 I $\mathrm{P}$ a g e 


\section{Chapter 3}

\section{Uniquely sized nanogels via crosslinking polymerization}

Nanogels are very promising carriers for nanomedicine, as they can be prepared in the favorable nanometer size regime, can be functionalized with targeting agents and are responsive to stimuli, i.e. temperature and $\mathrm{pH}$. This induces shrinking or swelling, resulting in controlled release of a therapeutic cargo. Our interest lies in the controlled synthesis of functional nanogels, such as those containing epoxide moieties, that can be subsequently functionalized. Co-polymerization of glycidyl methacrylate and a bifunctional methacrylate crosslinker under dilute conditions gives rise to well-defined epoxidefunctional nanogels, of which the sizes are controlled by the degree of polymerization. Nanogels with well-defined sizes (polydispersity of 0.2) ranging from $38 \mathrm{~nm}$ to $95 \mathrm{~nm}$ were prepared by means of controlled radical polymerization. The nanogels were characterized in detail by FT-IR, DLS, size exclusion chromatography, NMR spectroscopy, AFM and TEM. Nucleophilic attack with functional thiols or amines on the least hindered carbon of the epoxide provides water-soluble nanogels, without altering the backbone structure, while reaction with sodium azide provides handles for further functionalization via click chemistry.

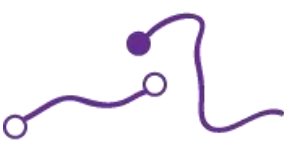

Monovalent monomer \& divalent crosslinker

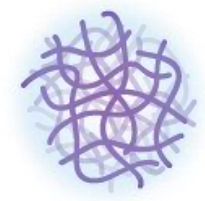

Nanogel network
Functionalization with a

radio label $\mathrm{C}$

a pharmaceutical

and a targeting label 0

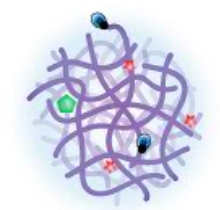

Loaded nanogel carrier 


\subsection{Introduction}

The emergence of nanomedicine brought about personalized medicine by combining nanotechnology and medicine. ${ }^{1-2}$ The field of theranostics applies nano-sized macromolecules to combine controlled administration of therapeutic drugs with diagnostics. ${ }^{3}$ Polymer nanoparticles are particularly interesting to this end as their physical properties, structure and composition can be controlled. ${ }^{4}$ These nanoparticles are readily rendered hydrophilic and biocompatible, and can be designed to encapsulate and release therapeutics. ${ }^{5}$ Polymer nanoparticles such as nano-sized hydrogels, also coined nanogels - are crosslinked, spherical, three-dimensional soft materials that are highly modular in nature. Nanogels displaying high water content, large surface area, high loading capacity, internal network, good stability in biological fluids and responsiveness to external stimuli. ${ }^{6-8}$ Desirable properties such as biodegradability, blood compatibility and amphiphilicity can be built in. ${ }^{9-10}$ The size, surface charge, porosity, softness and density can be tuned. As such nanogels have found use in tissue engineering, 7, 11-12 vaccines, cancer treatment, ${ }^{13-16}$ bioimaging, ${ }^{17-18}$ antifouling, ${ }^{19-20}$ ophthalmic diseases, ${ }^{21}$ biosensing, ${ }^{18,22-28}$ pain management, ${ }^{29}$ and nucleic acid delivery systems.

There are several ways to synthesize nanogels, such as emulsion polymerization, precipitation polymerization, ${ }^{30-31}$ inverse nanoprecipitation, ${ }^{32-33}$ self-assembly ${ }^{9}$ and template assisted polymerization ${ }^{34}$. To achieve optimal cellular uptake and biodistribution, nanoparticles should be between $10-100 \mathrm{~nm}$ in diameter. ${ }^{35-36}$ Others have succeeded in forming nanogels of well-defined uniform size. ${ }^{37}$ However their strategies either require multiple steps to form nanogels ${ }^{38-40}$, surfactants $s^{41-42}$ or produce a single size per reaction performed. ${ }^{43}$ Controlled crosslinking polymerization methods with multifunctional monomers provide excellent control over the resulting nanogels, as reaction conditions such as reaction time and monomer concentration determine the size of the nanogel. ${ }^{44}$ Controlled radical polymerization techniques, such as ATRP and RAFT 
slow the reaction rate to promote the formation of uniform particles. ${ }^{45-46}$ These strategies, that provide easy access to well-defined polymeric nanoparticles with uniform composition over the entire 10 - $100 \mathrm{~nm}$ size regime, have been reported for core-shell nanogels and hyperbranched polymers ${ }^{47-48}$ but not for nanogels. Good control over nanogel size is highly desirable in biomedical applications as it determines ease of endosomal escape. ${ }^{49-51}$

Cellular uptake and biodistribution behavior of nanogels is strongly affected by the type of surface functionality. ${ }^{5}$ Factors such as polarity and surface charge determine the hydrophilicity of the nanogel as well as its circulation in blood. ${ }^{52}$ Other structural properties such as useful functional groups aid the attachment of targeting molecules and contrast agents. ${ }^{53}$ Synthesizing nanogels with built in handles provides a versatile template able to be modified to functional nanogels, such as vaccine transport vehicles. ${ }^{6}$, ${ }^{54}$ Epoxides are highly reactive groups resulting from the three-membered ring with high ring strain. ${ }^{55}$ As such epoxides readily undergo ring-opening reactions with a wide array of nucleophiles. ${ }^{56}$

In this work, we describe the development of uniquely sized epoxide-functional precursor nanogels via controlled crosslinking polymerization. In a subsequent postformation step, the epoxide moieties are functionalized with various amines, azides and thiols, as well as hydrolyzed to the corresponding diol. This provides a platform for the formation of versatile functional nanogels as carriers. 


\subsection{Results and Discussion}

\subsubsection{GMA-EGDMA nanogel synthesis}

GMA-EGDMA nanogels (NGs) were prepared by RAFT copolymerization of glycidyl methacrylate (GMA) and ethylene glycol dimethacrylate (EGDMA) in a 3:1 mole ratio under dilute conditions (5 wt\%).
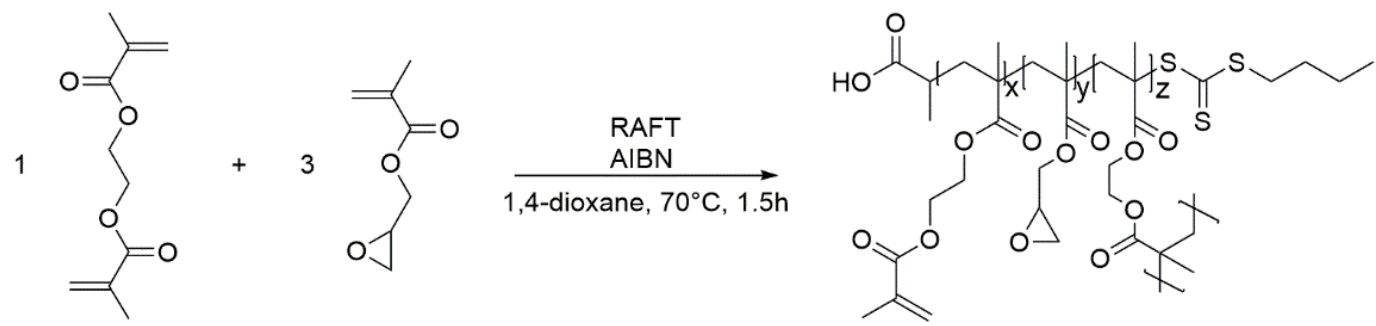

Scheme 1. The RAFT polymerization of EGDMA with GMA forming GMA-EGDMA nanogels.

Monomer conversion was determined by ${ }^{1} \mathrm{H}$ NMR spectroscopy, by integrating the characteristic signals of the alkene groups located at $\delta=6.1$ and $5.7 \mathrm{ppm}$. NG size was determined by dynamic light scattering (DLS) and Figure 1 shows the NG diameter as a function of conversion, revealing gradual NG growth. All NGs had a PDI of 0.2. Branching and pre-NG clusters are formed starting from $0 \%$ conversion up to $20 \%$ (for this particular system). Afterwards well-defined NGs are formed that continue growing while maintaining narrow dispersity. ${ }^{57}$ Eventually stagnation in particle size occurs at higher monomer conversions, followed by the formation of a macrogel. Four representative samples were prepared and studied with $21,26,42$ and $47 \%$ conversion, respectively, labeled GMA-EGDMA-21 and further. 


\subsubsection{GMA-EGDMA nanogel characterization}

Size exclusion chromatography (SEC) analysis on GMA-EGDMA NGs revealed broad size distributions that shift to shorter elution times with increasing monomer conversion. Due to the branches and crosslinks in the prepared NGs, the elution times cannot be related to linear polymer standards. Moreover, although the exclusion limit of the employed SEC column is over $4 \times 10^{7} \mathrm{~g} / \mathrm{mol}$, the shape of the distribution indicates that the NGs surpass the exclusion limits.
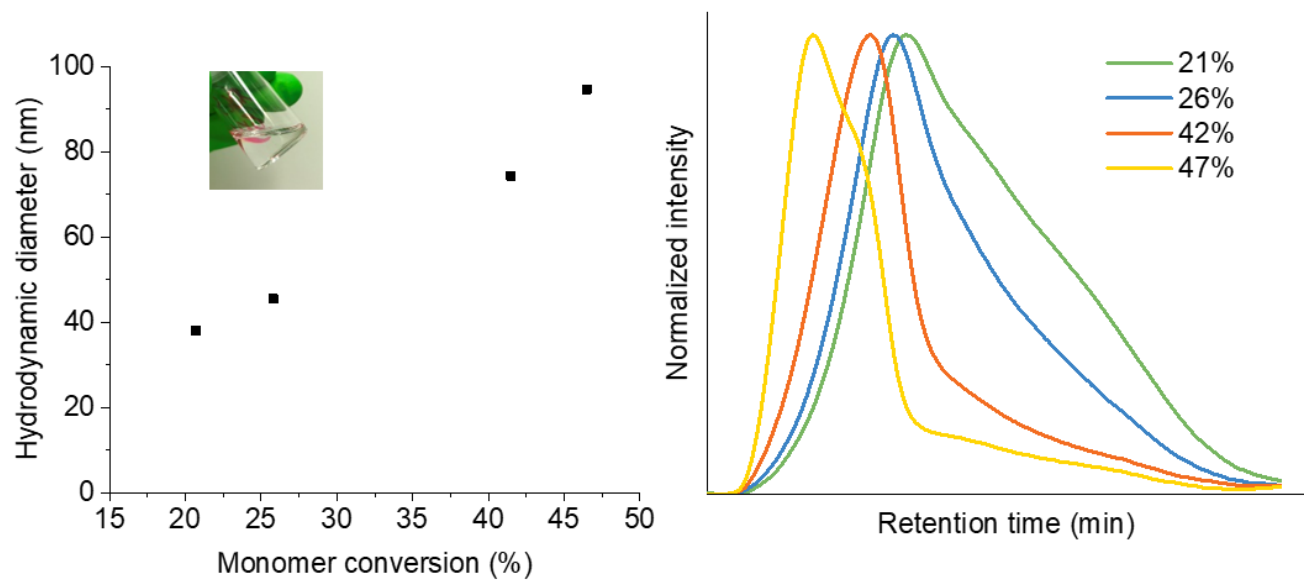

Figure 1. Hydrodynamic diameter of GMA-EGDMA NGs as a function of monomer conversion. Inset: GMAEGDMA in 1,4-dioxane solution (left). Size exclusion chromatography traces of GMA-EGDMA NGs at increasing monomer conversion (right).

The morphologies of GMA-EGDMA NGs were further analyzed by microscopy techniques. The TEM and AFM images of GMA-EGDMA-47 is highlighted below in Figure 2. Analysis of the TEM image gave a mean diameter of $17 \mathrm{~nm}$. AFM indicated a mean diameter of 20 $\mathrm{nm}$ and a mean particle height of $3 \mathrm{~nm}$. These results combined the with DLS data in Figure 1 demonstrate the malleability and responsiveness of the synthesized NGs. These NGs 
swell in solution becoming spherical in shape (DLS). Once casted onto a surface and dried, the NGs flatten out and take up a disc-like shape. ${ }^{58}$ This phenomenon is observed both at ambient pressure (AFM) as well as under vacuum (TEM).
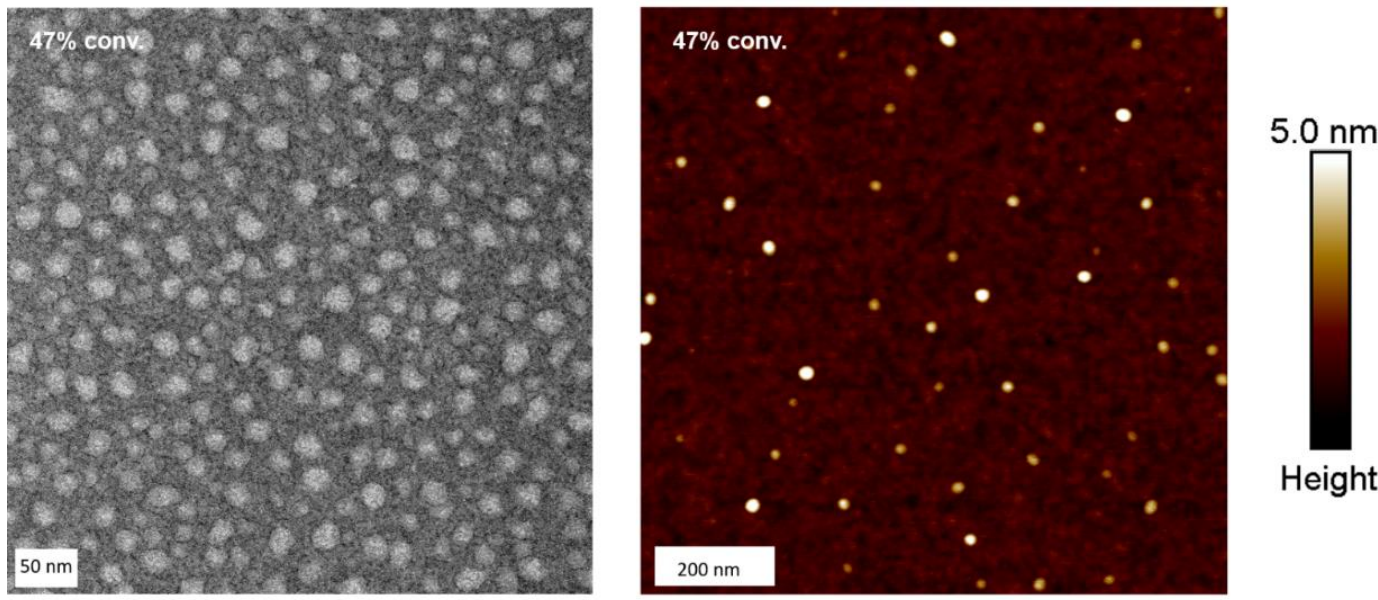

Height

Figure 2. TEM image of GMA-EGDMA NG (on the left) displaying round particles with a mean diameter of 17 $\mathrm{nm}$. AFM image (on the right) likewise indicates the formation of round, disc-like particles with a mean diameter of $20 \mathrm{~nm}$ and a mean height of $4 \mathrm{~nm}$. The scale bars are $50 \mathrm{~nm}$ and $200 \mathrm{~nm}$ for TEM and AFM, respectively.

${ }^{1} \mathrm{H}$ NMR spectroscopy on GMA-EGDMA NG, reveals characteristic signals corresponding to the epoxide moieties, from which a concentration of residual methacrylates of 1.10 $\mathrm{mmol} / \mathrm{g}$ is calculated. Comparison of the signals at $\delta=4.3 \mathrm{ppm}(4 \mathrm{H}$ from EGDMA and $1 \mathrm{H}$ from GMA) to those at $3.8 \mathrm{ppm}(1 \mathrm{H}$ from GMA), gives an EGDMA:GMA incorporation ratio of approximately 1:3, which is in accordance with the feed ratio. 


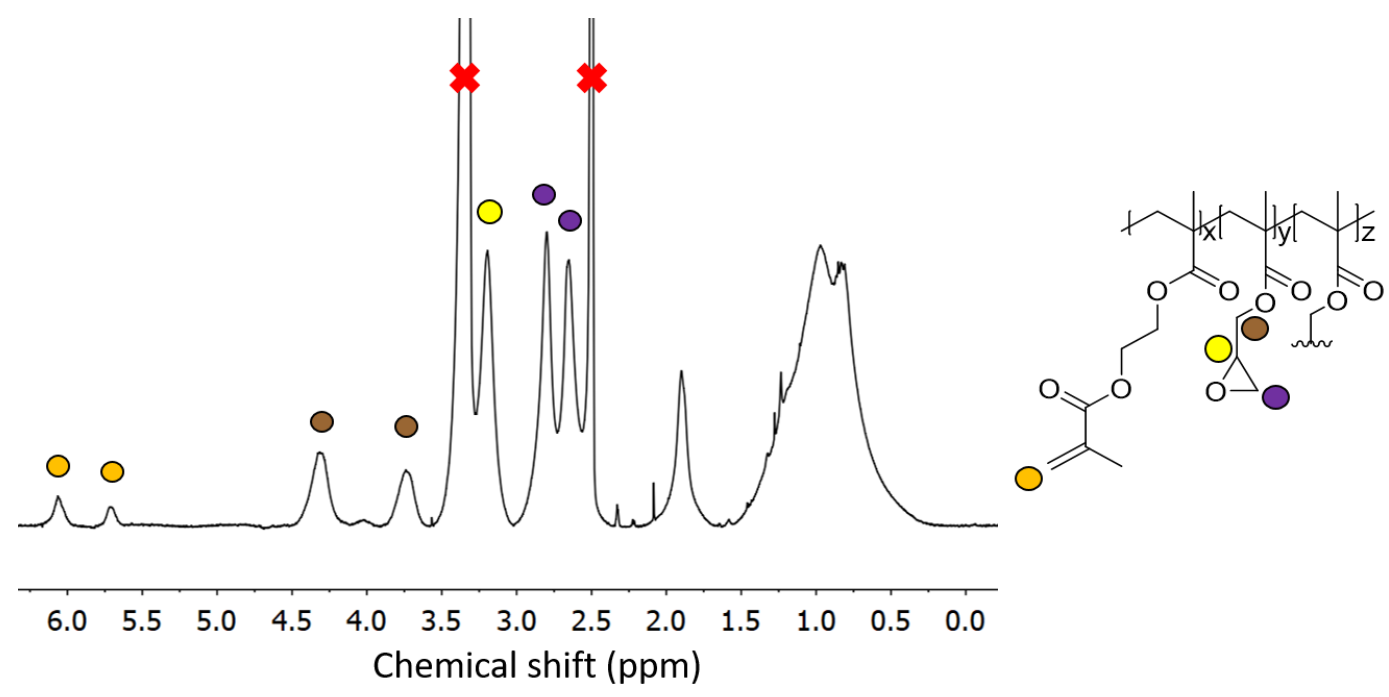

Figure 3. ${ }^{1} \mathrm{H}$ NMR spectroscopy of a representative GMA-EGDMA sample at $29 \%$ monomer conversion, with characteristic epoxide signals at $\delta=3.2 \mathrm{ppm}, 2.8 \mathrm{ppm}$ and $2.7 \mathrm{ppm}$ in DMSO- $\mathrm{d}_{6}$.

NG formation also proceeds as described above in the presence of a third monomer. In the case of a monomer with a methacrylate functionality such as DMAEMA, it will be covalently incorporated into the NG structure.

As known from the literature, NGs and other nanoparticles often encounter stability issues, resulting in aggregation. ${ }^{59}$ Particles with reactive groups tend to crosslink, while dried solid particles may no longer be redispersed. To assess the stability of the NGs formed, DLS measurements were repeated over time. The dried NGs were readily dissolved in various organic solvents (DMF, DMSO, 1,4-dioxane, $\mathrm{CHCl}_{3}, \mathrm{DCM}$, acetone) and DLS analysis did not show signs of degradation or decomposition (no changes in hydrodynamic diameter or PDI) even after storing for 6 months at room temperature in dried state. 


\subsubsection{GMA-EGDMA nanogel functionalizations}

Having demonstrated the controllability and robustness of this system we proceeded to functionalization of the epoxide moiety by exploiting its reactivity and ring strain to demonstrate the versatility of these NGs. ${ }^{60}$ All functionalizations and reactions mentioned hereafter were done on GMA-EGDMA NG of which the remaining methacrylates were quenched with excess AIBN. The hydrodynamic particle diameter of these NGs was $41 \mathrm{~nm}$ as determined by DLS in 1,4-dioxane.

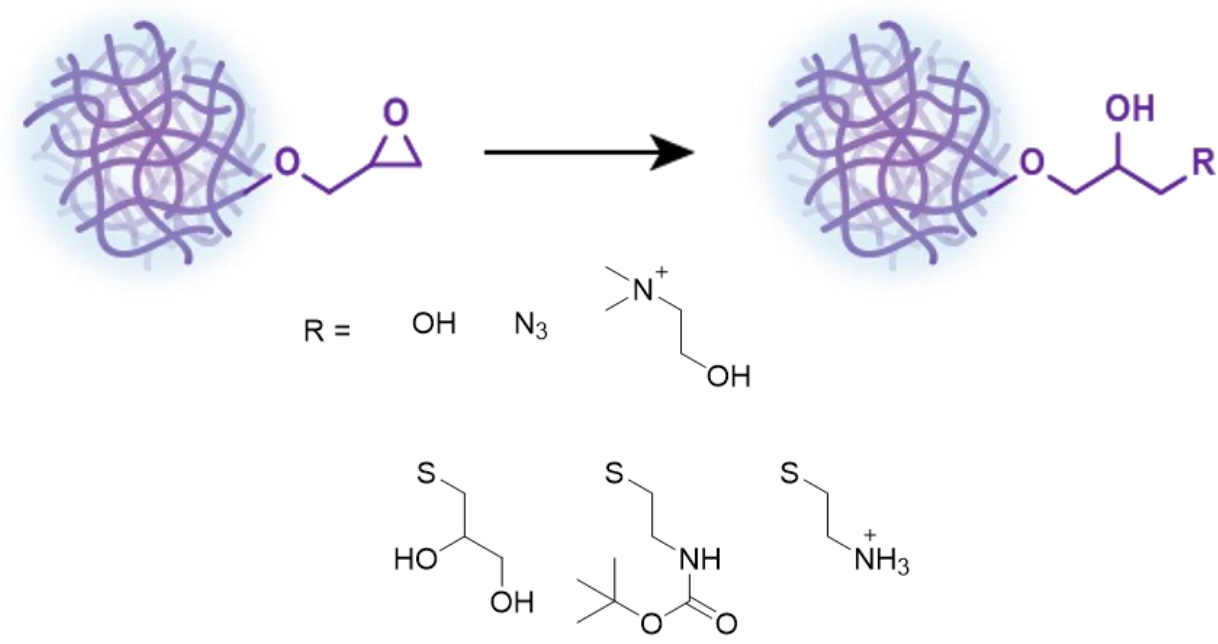

Scheme 2. Functionalization of GMA-EGDMA NG through acid catalyzed epoxide ring opening, ring opening with sodium azide or tertiary amines, and ring opening with functional thiols.

The epoxide moieties on the NGs were hydrolyzed in order to render the NGs watersoluble. Reaction with excess TFA and water at room temperature resulted in GMAEGDMA-OH NG. ${ }^{1} \mathrm{H}$ NMR spectroscopy no longer displayed the characteristic epoxide signals, indicating complete conversion, while new signals at $\delta=4.7$ and $4.9 \mathrm{ppm}$ are in 
agreement with alcohol groups on the hydrolyzed epoxide. However, the solubility of GMA-EGDMA-OH NG in water remained limited. Foaming is observed, indicating solvation in water. However, even at concentrations as low as $0.12 \mathrm{mg} / \mathrm{mL}$ some sedimentation was observed. We suspect that there are two reasons for this phenomenon. Weaver and coworkers demonstrated that degree of polymerization (DP) plays a significant role in water-solubility of HEMA homopolymers bearing pendant hydroxyl groups like GMA-EGDMA-OH. ${ }^{61}$ The DP of the sedimented GMA-EGDMA-OH particles was likely too high rendering these NGs insoluble in water. Likewise, the ratio between the hydrophilic hydroxyl groups and the hydrophobic crosslinker and backbone of the sedimented NGs is too low to properly solvate these NGs. The variations in solubility indicate the formation of slightly different NG particles. Those NGs containing a higher ratio of epoxide moieties and thus more hydroxyl groups after hydrolysis are watersoluble, whereas those with fewer epoxide groups remain insoluble in water after hydrolysis. Likewise, GMA-EGDMA-OH NGs with a lower DP are water-soluble. DLS measurements in DMF gave a particle diameter of $104 \mathrm{~nm}$. Such a significant increase in size is expected for a more polar NG in a polar solvent with intrinsic swelling abilities. The increase in PDI further suggests the formation of slightly different particles. Various amounts of epoxide moieties and thus hydroxide groups after hydrolysis, mean varying amounts of swelling, resulting in a variety of GMA-EGDMA-OH particle sizes and thus an increase in PDI. 


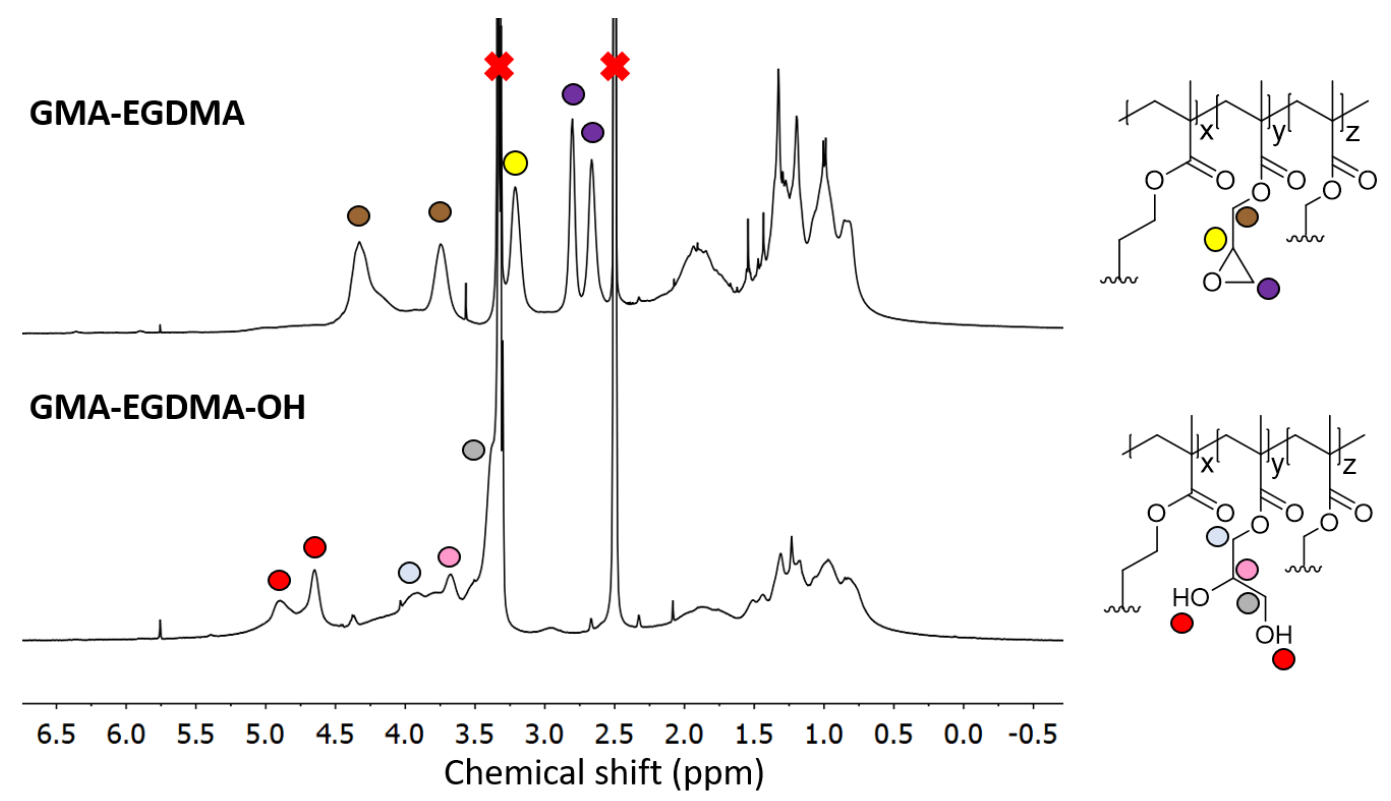

Figure 4. ${ }^{1} \mathrm{H}$ NMR spectroscopy of GMA-EGDMA-OH NG (bottom) indicating full conversion as well as the presence of hydroxyl groups at $\delta=4.9 \mathrm{ppm}$ and $\delta=4.7 \mathrm{ppm}$.

Khan and coworkers have extensively researched the opening of epoxides with thiols, utilizing LiOH as a catalyst. ${ }^{62-66}$ To further probe the water-solubility of these NGs, the choice was made for 1-thioglycerol as the thiol reagent, forming GMA-EGDMA-THG NG. Stoichiometric amounts of $\mathrm{LiOH}$ were needed to achieve full conversion. ${ }^{1} \mathrm{H}$ NMR spectroscopy indicates complete conversion and a successful reaction. DLS measurements in DMSO gave a Z-average of $67 \mathrm{~nm}$. Despite full conjugation of 1thioglycerol onto the epoxide moieties, the resulting NG did not become appreciably water-soluble. We suspect that the ratio between the alcohol groups and the hydrophobic EGDMA crosslinker and methacrylate-based polymer backbone is too low to achieve (complete) water-solubility. 
Alternatively, water-solubility can be achieved by introducing charge. GMA-EGDMA-Boc NG was synthesized as 2-(Boc-amino)ethanethiol has a recognizable signal on ${ }^{1} \mathrm{H}$ NMR spectroscopy as well as the added benefit of becoming water-soluble upon deprotection. ${ }^{1} \mathrm{H}$ NMR spectroscopy of GMA-EGDMA-Boc NG indicates complete conversion. DLS measurements in DCM gave a Z-average of $75 \mathrm{~nm}$. The increase in NG size is likely due to the bulky Boc group. Boc-deprotection was achieved with excess TFA resulting in GMAEGDMA-NH ${ }_{3}{ }^{+} \mathrm{NG}$, as confirmed by the disappearance of the ${ }^{1} \mathrm{H}$ NMR signal at $\delta=1.4 \mathrm{ppm}$ corresponding to the 9 protons from the Boc group (data not shown). This cationic NG is fully water-soluble, with a hydrodynamic particle diameter of $91 \mathrm{~nm}$ in $\mathrm{H}_{2} \mathrm{O}$ and a high surface charge of $+37 \mathrm{mV}$ in $10 \mathrm{mM}$ HEPES solution.

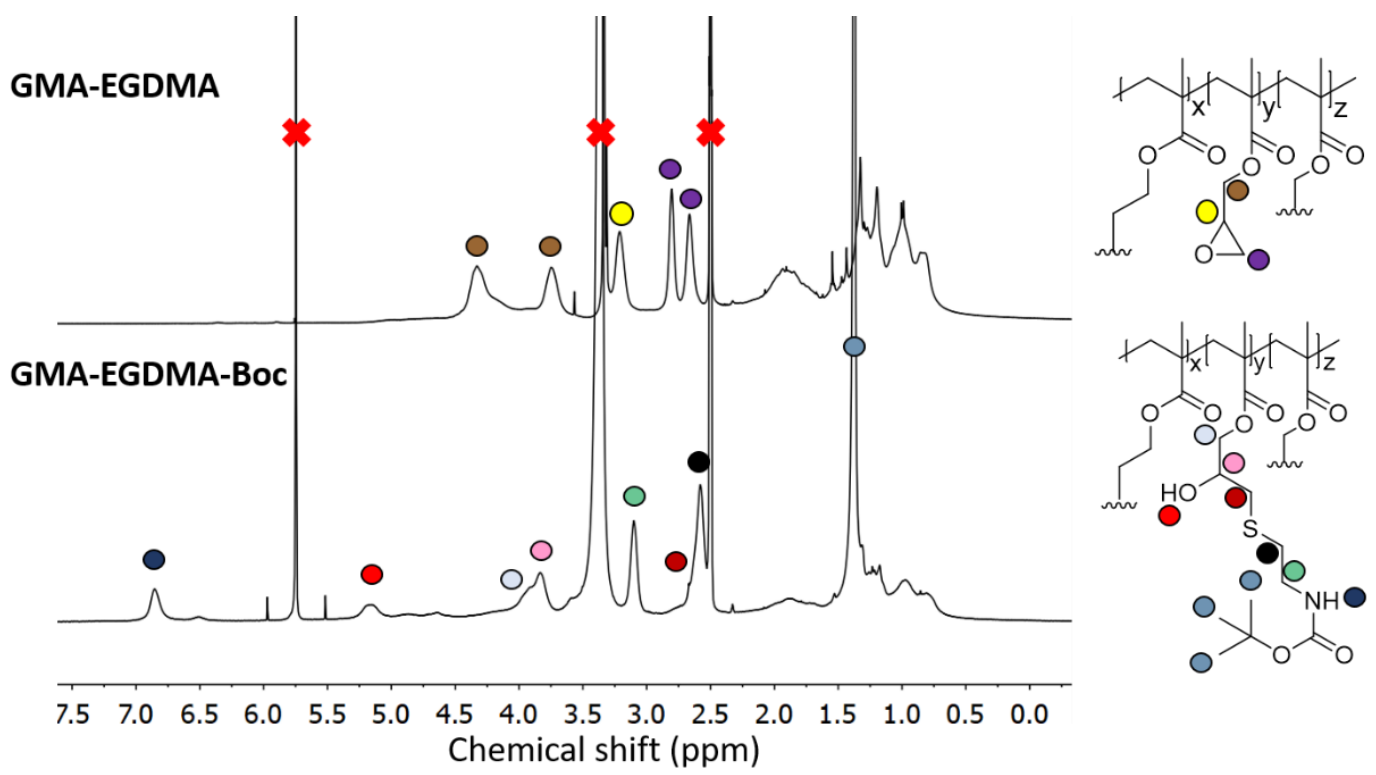

Figure 5. ${ }^{1} \mathrm{H}$ NMR spectra of NGs before (GMA-EGDMA) and after conjugation of Boc-aminoethanethiol (GMA-EGDMA-Boc). 
Amines are also capable of nucleophilic attack on an epoxide and have been successfully used in post-polymerization functionalization. $\mathrm{Xu}$ and coworkers functionalized pGMA with various amines to synthesize nonviral transfection vectors for gene therapy. ${ }^{67-71}$ Literature indicates however that crosslinking occurs most likely due to further functionalization of the amine. ${ }^{72-74}$ As such a tertiary amine was chosen for the formation of GMA-EGDMA-NR ${ }_{3}{ }^{+}$NG. ${ }^{1} \mathrm{H}$ NMR spectroscopy exhibits no epoxide signals, indicating complete conversion. A signal at $\delta=3.3 \mathrm{ppm}$ corresponds to the methyl groups on the quaternized amine. This cationic NG is water-soluble and displays a highly positive surface charge of $+30 \mathrm{mV}$ in $10 \mathrm{mM}$ HEPES. DLS measurements in $\mathrm{H}_{2} \mathrm{O}$ gave a Z-average of $170 \mathrm{~nm}$ indicating characteristic swelling in water. ${ }^{75}$

Tsarevsky and coworkers provide a protocol for the functionalization of the glycidyl methacrylate epoxide with sodium azide. ${ }^{76}$ Azides are a popular functional group as they can undergo azide-alkyne click reactions with various alkynes, installing radioactive tracers, targeting ligands and more. Ammonium chloride is utilized to protonate the alkoxide anion, preventing any possible crosslinking. ${ }^{77}$ The ${ }^{1} \mathrm{H}$ NMR spectrum of GMAEGDMA-N ${ }_{3}$ NG displays no epoxide signals at $\delta=3.2 \mathrm{ppm}, 2.8 \mathrm{ppm}$ and $2.7 \mathrm{ppm}-$ indicating complete conversion. The signals for the methylene adjacent to the azide group $\left(-\mathrm{CH}_{2} \mathrm{~N}_{3}\right)$ can be observed at $3.3 \mathrm{ppm} .{ }^{1} \mathrm{H}$ NMR spectroscopy was also conducted in DMF$d_{7}$. The resulting spectra are comparable to those found in literature. ${ }^{76}$ The ${ }^{13} \mathrm{C}$ NMR spectroscopy signal indicative of a carbon next to the azide moiety $\left(\mathrm{CH}_{2} \mathrm{~N}_{3}\right)$ can be observed at $\delta=53 \mathrm{ppm}$ in DMSO-d $\mathrm{d}_{6}$ and at $\delta=55 \mathrm{ppm}$ in DMF-d $\mathrm{d}_{7}$ (Figures S1 and S2 respectively in supplementary data). In addition, a distinctive azide signal is observed by FT-IR at $2104 \mathrm{~cm}^{-1}$. A broad peak at $3400 \mathrm{~cm}^{-1}$ corresponding to the hydroxyl group further confirms successful epoxide opening. 


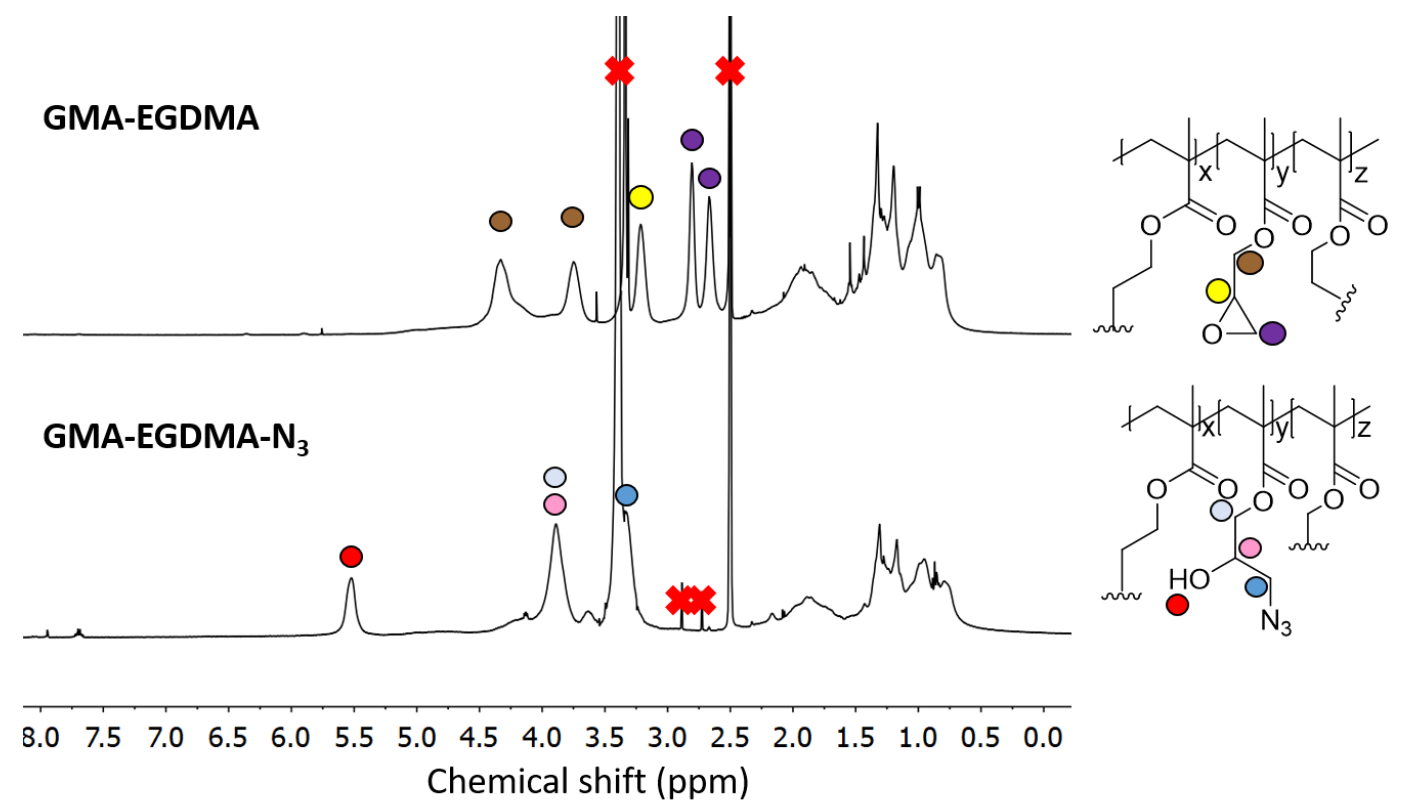

Figure 6. The ${ }^{1} \mathrm{H}$ NMR spectrum of GMA-EGDMA-N $\mathrm{N}_{3}$ (bottom) in DMSO- $\mathrm{d}_{6}$ indicates no more epoxides present and thus full conversion.

Partial epoxide opening was also carried out with 0.5 equivalents of sodium azide to assess quantitative reactivity and control over functionalization. The FT-IR spectrum in Figure 7 (middle) displays an azide peak at $2104 \mathrm{~cm}^{-1}$, an $\mathrm{OH}$ peak at $3370 \mathrm{~cm}^{-1}$ and $\mathrm{C}=\mathrm{O}$ peak at $1721 \mathrm{~cm}^{-1}$. The residual epoxide peak at $907 \mathrm{~cm}^{-1}$ is also present. ${ }^{1} \mathrm{H}$ NMR spectroscopy in DMSO- $d_{6}$ indicated approximately $43 \%$ conversion which is lower than the targeted $50 \%$ conversion. This is likely due to a stoichiometric excess needed to drive the reaction forward as well as the error margin of calculations done on NMR spectra of nanoparticles. 
In a subsequent reaction the azide groups were clicked with a pro-fluorescent coumarin dye containing an alkyne moiety to assess their accessibility and reactivity when forming part of a complex NG network.
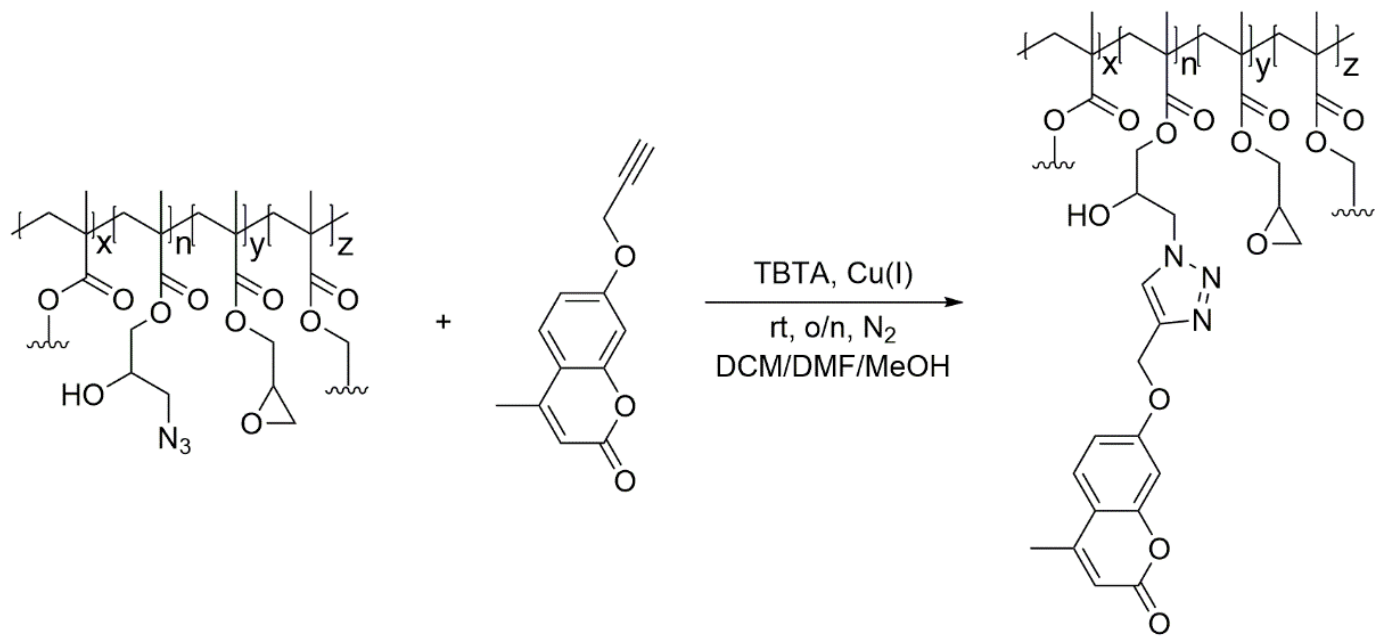

Scheme 3. GMA-EGDMA-N $\mathbf{N}_{3}$ NGs with residual epoxide groups were clicked with alkyne-coumarin under inert conditions. Tetrakis(acetonitrile)copper(I) hexafluorophosphate and TBTA (tris((1-benzyl-4triazolyl)methyl)amine) catalyzed the reaction.

The FT-IR measurements depicted in Figure 7 indicate the disappearance of the azide peak at $2102 \mathrm{~cm}^{-1}$ (bottom spectrum). It is suspected that the triazole peak is located at 1650 $\mathrm{cm}^{-1}$ but is shielded by the large carbonyl peak. ${ }^{78}$ The spectra in Figure 8 display the significant increase in fluorescence of the NG after attachment of the fluorescent molecule. 


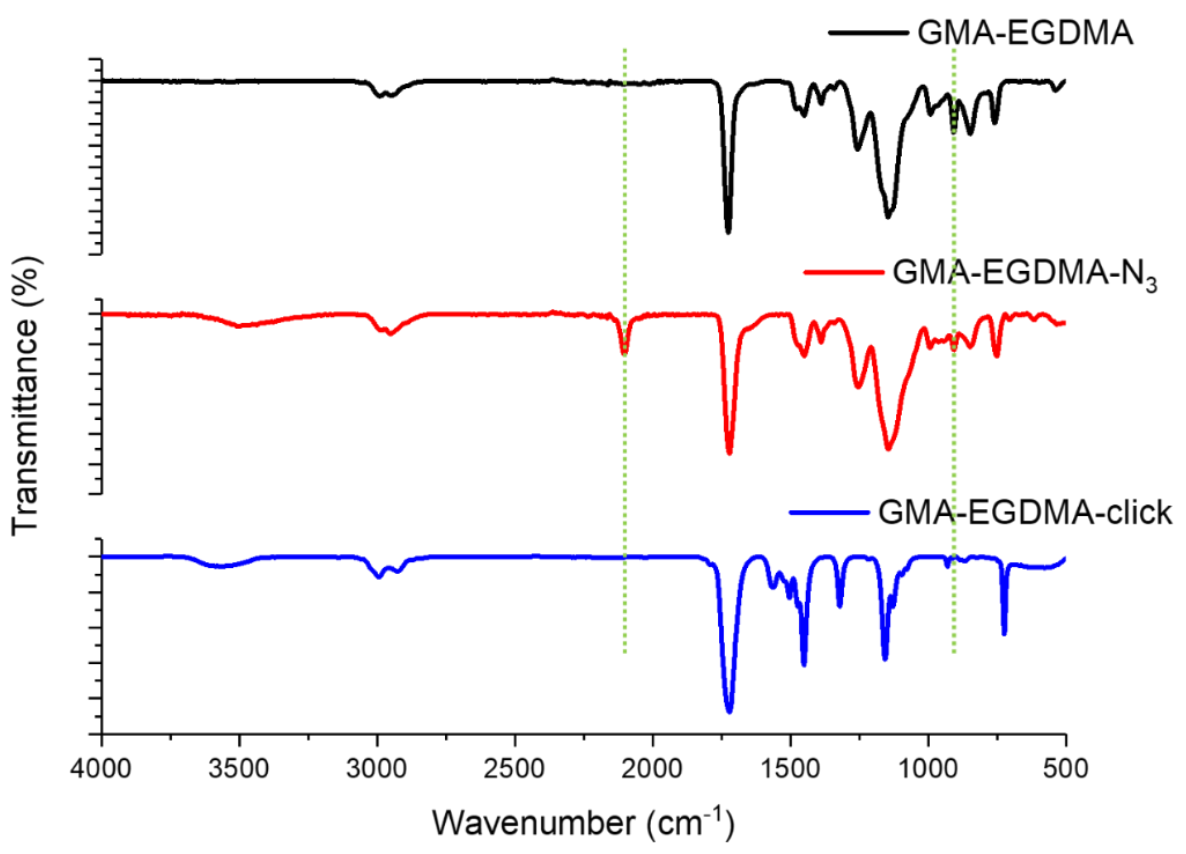

Figure 7. The. The top IR spectrum displays the GMA-EGDMA NG prior to undergoing post-synthesis modifications, displaying an epoxide peak at $907 \mathrm{~cm}^{-1}$. In the middle spectrum the emergence of a hydroxyl group can be seen at $3400 \mathrm{~cm}^{-1}$ as well as an azide group at $2102 \mathrm{~cm}^{-1}$ after reacting with sodium azide. A subsequent click reaction of the azide moieties results in the disappearance of peak at $2102 \mathrm{~cm}^{-1}$ as can be seen in the bottom spectrum. 


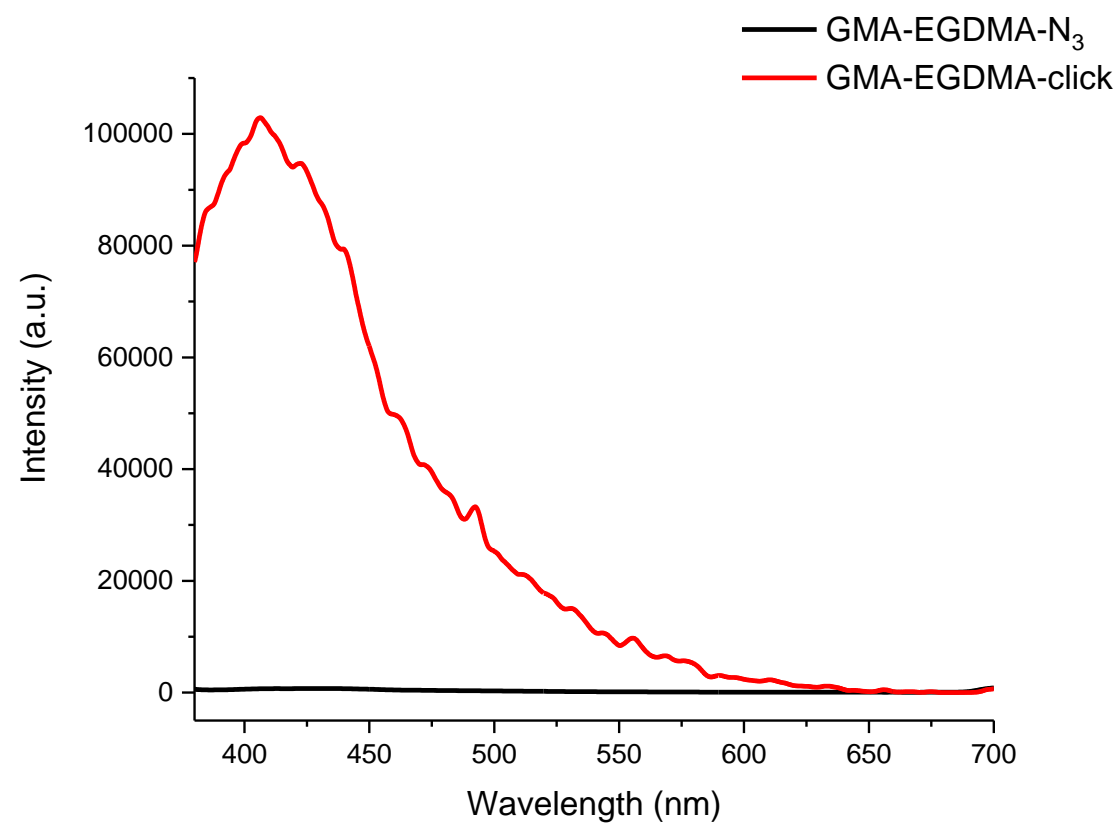

Figure 8. Comparing the fluorescence spectra of GMA-EGDMA-N $\mathrm{N}_{3}$ before and after the reaction with a profluorescent coumarin dye indicates a significant increase in fluorescence intensity. Both NGs were measured at $3 \mathrm{mg} / \mathrm{mL}$ in DMF, exciting at $\lambda=360 \mathrm{~nm}$ and detecting the emission at $\lambda=380 \mathrm{~nm}-700 \mathrm{~nm}$.

\subsection{Conclusions}

In this chapter, we describe a novel method for the preparation of epoxide containing NGs that are robust and size-controllable. GMA-EGDMA NGs can be synthesized with a particle diameter between 38 and $95 \mathrm{~nm}$. The polydispersity witnessed on TEM and GPC suggests that this method is quite sensitive and requires fine-tuning. Computational models and kinetics experiments should further elucidate intricacies influencing steady NG growth and reproducibility. Nevertheless, NGs formed via this method are highly robust and stable. They can withstand freeze-drying and solvent removal in vacuo and 
once redissolved months later, retain their original size. The epoxide moiety incorporated in the NG remains accessible and can undergo post-polymerization modifications with amines, thiols, TFA and azides to a wide variety of versatile functional NGs. An increase in PDI of the resulting NGs suggests that some reactions are better tolerated than others. Water-soluble NGs are easily achieved by introducing permanent charge. The formation of charged NGs allows modification of its surface polarity and thus its efficacy in blood. This work serves to further illustrate the versatility achievable with polymeric NGs combined with increased efficiency and control when synthesized in a single step via a living polymerization with monovalent and divalent vinyl monomers.

\subsection{Acknowledgements}

Rachèl Elzes is thanked for her unwavering help and support during nanogel synthesis. Dr. E. G. Keim is acknowledged for his help with TEM measurements. Dr. Michel Klein Gunnewiek is acknowledged for his help with AFM measurements.

\subsection{Experimental section}

Materials. Glycidyl methacrylate (GMA, 97\%) and ethylene glycol dimethacrylate (EGDMA, 98\%) were purchased from Sigma-Aldrich and passed through a column of neutral $\mathrm{Al}_{2} \mathrm{O}_{3}$ prior to use. $\mathrm{N}, \mathrm{N}$-dimethylformamide (DMF, anhydrous, 99.8\%), trifluoroacetic acid (TFA, HPLC grade, >99.0\%), N, N-dimethylethanolamine (redistilled, 99.5\%), 1,4-dioxane (HPLC grade, >99.5\%), azobisisobutyronitrile (AIBN, 98\%), 2-(Bocamino)ethanethiol (97\%), lithium hydroxide (LiOH, reagent grade, 98\%), tris((1-benzyl-4triazolyl)methyl)amine (TBTA, 97\%) and tetrakis(acetonitrile)copper(I) hexafluorophosphate $\left(\mathrm{Cu}\left(\mathrm{CH}_{3} \mathrm{CN}\right)_{4} \mathrm{PF}_{6}, 97 \%\right)$ were purchased from Sigma-Aldrich and used 
without further purification. Sodium azide $\left(\mathrm{NaN}_{3}, 99 \%\right)$ was purchased from Acros Organics and used as supplied. Safety note: Large scale reactions of azide are prone to dangerous exothermic reactions. The use of chlorinated solvents in the presence of sodium azide must be avoided, as the byproducts may be highly explosive. ${ }^{79}$ 4-methyl-7(prop-2-yn-1-yloxy)-2H-chromen-2-one was purchased from MolPort as used without any further purifications. Dichloromethane (DCM, 99.7\%) and methanol (MeOH) were obtained from Ossum Chemicals. Demi-water was used unless stated otherwise. The RAFT agent 2-[[(butylthio)thioxomethyl] thio] propanoic acid was synthesized following a literature procedure. ${ }^{80}$

Analyses. Monomer conversions were determined by ${ }^{1} \mathrm{H}$ NMR spectroscopy in DMSO- $\mathrm{d}_{6}$ or $\mathrm{CDCl}_{3}$ on a Bruker Avance $400 \mathrm{MHz}$ spectrometer. The signals of the deuterated solvents were used as a reference. Monomer conversions were calculated either by preparing an ${ }^{1} \mathrm{H}$ NMR sample with $20 \mathrm{mg} \mathrm{NG}, \mathrm{CDCl}_{3}$, along with $25 \mu \mathrm{L}$ of DMF as internal standard or by collecting the unreacted monomers during precipitation. Hydrodynamic diameters were measured by dynamic light scattering (DLS) using a Malvern Instruments Zetasizer ZS at a backscatter angle of $173^{\circ}$ and taking the Z-average in intensity. Measurements were done in triplicate, employing PEG/PiBMA as control. Zeta potential was measured on the same equipment at $20^{\circ} \mathrm{C}$ in water employing folded capillary cells. Infrared spectroscopy (FT-IR) was performed on a Bruker Alpha and analyzed with OPUS software. Transmission electron microscopy (TEM) samples were prepared with a concentration of $0.8 \mathrm{mg} / \mathrm{mL}$ in 1,4-dioxane, of which $5 \mu \mathrm{L}$ was casted on a copper grid. The sample was subsequently stained with a $1 \mathrm{w} / \mathrm{v} \%$ MilliQ solution of uranyl acetate for 35 seconds. Excess staining was removed with a filter paper. TEM images were collected on a Philips CM300ST-FEG transmission electron microscope 300 $k V$ equipped with GATAN Ultrascan 1000 (2kx2k CCD camera) and GATAN Tridiem energy filter (2Kx2K CCD camera). The TEM images were analyzed with ImageJ software by 
measuring the diameters of 200 individual NG spheres and calculating the average size and standard deviation. Dialysis was done using Spectra/Por 6 Dialysis Membrane Prewetted RC Tubing with a molecular weight cut-off of $1 \mathrm{kD}$. Size exclusion chromatography (SEC) was performed on a Waters e2695 Separations Module equipped with an Agilent PLgel $20 \mu \mathrm{m}$ MIXED-A 300x7.5 mm column, a Waters photodiode array detector (PDA 2998), a fluorescence detector (FLR 2475) and a refractive index detector (RI 2414). Samples were dissolved in HPLC grade chloroform (eluent) to a concentration of 2.0 $\mathrm{mg} / \mathrm{mL}$ and filtered with a GE Healthcare Whatman SPARTAN 13/0.2 RC $0.2 \mu \mathrm{m}$ syringe filter. An injection volume of $100 \mu \mathrm{L}$ was applied along with an elution speed of $1 \mathrm{~mL} / \mathrm{min}$ at $35^{\circ} \mathrm{C}$. Molecular weights and PDI were calculated from linear polystyrene calibration standards.

\subsubsection{Nanogel synthesis}

GMA-EGDMA NG formation. RAFT agent 2-[[(butylthio)thioxomethyl] thio] propanoic acid ( $248.8 \mathrm{mg}, 1 \mathrm{mmol}, 1$ equiv.), AIBN ( $34.3 \mathrm{mg}, 0.2 \mathrm{mmol}, 0.2$ equiv.), GMA (5.2 mL, $39.2 \mathrm{mmol}, 37.5$ equiv.), EGDMA ( $2.46 \mathrm{~mL}, 13.1 \mathrm{mmol}, 12.5$ equiv.) and 1,4dioxane (150 mL, $95 \mathrm{w} / \mathrm{w} \%$ ) were added to a $500 \mathrm{~mL}$ round bottom flask equipped with a stir bar, sealed with a septum and purged with nitrogen for 45 min. The flask was subsequently placed in an oil bath at $70^{\circ} \mathrm{C}$ and allowed to react for $1.5 \mathrm{~h}$ after which it was taken out of the oil bath and quenched with $3 \mathrm{~mL}$ DCM or excess AIBN (4.42 g, 26.9 $\mathrm{mmol}$ ) in 1,4-dioxane (30 mL). The NG was precipitated three times in hexane yielding a white solid (unquenched NG $2.06 \mathrm{~g}, 29 \%$ conversion, $5.51 \mathrm{mmol} / \mathrm{g}$ epoxides, 1.102 $\mathrm{mmol} / \mathrm{g}$ dangling methacrylates; quenched NG $2.78 \mathrm{~g}, 54 \%$ conversion, $3.04 \mathrm{mmol} / \mathrm{g}$ epoxides). 


\subsubsection{Nanogel functionalization}

GMA-EGDMA-N 3 NG formation. GMA-EGDMA NG (101 mg, 0.3 mmol epoxides), sodium azide ( $40.4 \mathrm{mg}, 0.6 \mathrm{mmol}, 2$ equiv.), ammonium chloride ( $49.2 \mathrm{mg}, 0.9 \mathrm{mmol}, 3$ equiv.) and DMF (10 mL) were combined in a $25 \mathrm{~mL}$ round bottom flask, equipped with a magnetic stir bar, and heated at $50^{\circ} \mathrm{C}$ for 2 days. The salts did not fully dissolve in DMF. The crude NG was precipitated in water, filtered, rinsed with water and transferred to a new filter paper to dry.

GMA-EGDMA-N 3 NG formation. GMA-EGDMA NG (96.7 mg, 0.3 mmol epoxides), sodium azide ( $9.4 \mathrm{mg}, 0.15 \mathrm{mmol}, 0.5$ equiv.), ammonium chloride ( $48.8 \mathrm{mg}, 0.9 \mathrm{mmol}, 3$ equiv.), and DMF (10 mL) were combined in a $25 \mathrm{~mL}$ round bottom flask, equipped with a magnetic stir bar and heated at $50{ }^{\circ} \mathrm{C}$ overnight. The salts did not fully dissolve in DMF. The crude NG was precipitated in water, filtered, rinsed with water and transferred to a new filter paper to dry resulting in a white solid ( $229 \mathrm{mg}, ~ 43 \%$ conversion).

GMA-EGDMA-click formation. A click procedure was used as described in literature. ${ }^{81}$ GMA-EGDMA-N $\mathbf{3}$ (10.15 mg, $\left.15.4 \mu \mathrm{mol}\right)$ was dissolved in DMF $(0.5 \mathrm{~mL})$ and stirred for $24 \mathrm{~h}$ to achieve maximum solubility. To a snap cap vial containing DCM (1.17 $\mathrm{mL}$ ) was added 4-methyl-7-(prop-2-yn-1-yloxy)-2H-chromen-2-one (3.28 mg, $15.3 \mu \mathrm{mol})$. TBTA (1.53 mg, $2.9 \mu \mathrm{mol})$ and $\mathrm{Cu}\left(\mathrm{CH}_{3} \mathrm{CN}\right)_{4} \mathrm{PF}_{6}(0.89 \mathrm{mg}, 2.4 \mu \mathrm{mol})$ were dissolved in $\mathrm{MeOH}$ (1.17 $\mathrm{mL}$ ) resulting in a light blue solution. $A \mathrm{~N}_{2}$ purged RB flask fitted with a $\mathrm{N}_{2}$ filled balloon, was charged with the NG solution, extra DMF $(0.5 \mathrm{~mL})$, the dye solution and the catalysts solution by means of $\mathrm{N}_{2}$ flushed syringes. The light green, turbid reaction mixture turned pale yellow during the $24 \mathrm{~h}$ reaction and remained turbid. Solvents were removed under reduced pressure resulting in a yellow/green solid. The product was redissolved in DMF, the solids were filtered out and the solution was subjected to flash column chromatography $\left(\mathrm{SiO}_{2}, \mathrm{DMF}\right)$. 
GMA-EGDMA-NR ${ }^{+}{ }^{+}$NG formation. GMA-EGDMA NG $(105.5 \mathrm{mg}, 0.3 \mathrm{mmol}$ epoxides), $\mathrm{N}, \mathrm{N}$-dimethylethanolamine ( $62 \mu \mathrm{L}, 0.6 \mathrm{mmol}, 2$ equiv.), DMF ( $9 \mathrm{~mL}$ ) and $\mathrm{H}_{2} \mathrm{O}(1$ $\mathrm{mL}$ ) were added to a $25 \mathrm{~mL}$ round bottom flask equipped with a magnetic stir bar and allowed to stir at $50^{\circ} \mathrm{C}$ for 2 days. The reaction mixture was precipitated in diethyl ether, dissolved in $\mathrm{H}_{2} \mathrm{O}$, dialyzed for 4 days against water, $\mathrm{MWCO}=1 \mathrm{kDa}$ and freeze-dried resulting in a white solid (40 $\mathrm{mg}, 100 \%$ conversion).

GMA-EGDMA-OH NG formation. GMA-EGDMA NG $(97.2 \mathrm{mg}, 0.3 \mathrm{mmol}$ epoxides), TFA ( $0.23 \mathrm{~mL}, 3 \mathrm{mmol}, 10$ equiv.), THF $(9 \mathrm{~mL})$ and $\mathrm{H}_{2} \mathrm{O}(1 \mathrm{~mL})$ were added to a $50 \mathrm{~mL}$ Erlenmeyer flask, equipped with a magnetic stir bar and allowed to stir overnight at room temperature. The solvents were removed in vacuo and the NG was redissolved in $\mathrm{MeOH}$ and precipitated in $\mathrm{H}_{2} \mathrm{O}$. The $\mathrm{NG}$ was dissolved in $\mathrm{H}_{2} \mathrm{O}$ and dialyzed against water for 2 days, MWCO = $1 \mathrm{kDa}$. Freeze drying resulted in a white solid $(100 \mathrm{mg}, 100 \%$ conversion).

GMA-EGDMA-THG NG formation. GMA-EGDMA NG $(96.8 \mathrm{mg}, 0.3 \mathrm{mmol}$ epoxides) and 1-thioglycerol ( $0.06 \mathrm{~mL}, 0.7 \mathrm{mmol}, 2.5$ equiv.) were dissolved in $9 \mathrm{~mL} \mathrm{DMF}$. $\mathrm{LiOH}$ (6.9 mg, $0.3 \mathrm{mmol}, 1$ equiv.) was dissolved in $1 \mathrm{~mL} \mathrm{H}_{2} \mathrm{O}$ and added dropwise to the organic solution. The cloudy reaction mixture stirred at room temperature overnight. The $\mathrm{NG}$ was dispersed in $\mathrm{H}_{2} \mathrm{O}$ and dialyzed against water for 3 days, $\mathrm{MWCO}=1 \mathrm{kDa}$. Freeze drying resulted in a white solid ( $130 \mathrm{mg}, 100 \%$ conversion).

GMA-EGDMA-Boc NG formation. GMA-EGDMA NG (94.7 mg, 0.3 mmol epoxides) and 2-(Boc-amino)ethanethiol $(0.12 \mathrm{~mL}, 0.7 \mathrm{mmol}, 2.5$ equiv.) were dissolved in $9 \mathrm{~mL}$ DMF. LiOH (24.7 mg, 1.0 mmol, 3.6 equiv.) was dissolved in $1 \mathrm{~mL} \mathrm{H} \mathrm{H}_{2} \mathrm{O}$ and added dropwise to the organic solution. The reaction mixture stirred overnight at room temperature becoming yellow and cloudy. The $\mathrm{NG}$ was dispersed in $\mathrm{H}_{2} \mathrm{O}$ and dialyzed against water for 6 days, MWCO $=1 \mathrm{kDa}$. Water was removed under reduced pressure. The NG was 
dissolved in $\mathrm{DCM}$, dried over $\mathrm{MgSO}_{4}$, filtered, concentrated in vacuo and precipitated in hexane twice.

GMA-EGDMA- $\mathrm{NH}_{3}{ }^{+}$NG formation. To a $12 \mathrm{~mL}$ colorless solution of GMA-EGDMABoc NG (0.29 mmol) in DCM was added TFA (0.11 mL, $1.4 \mathrm{mmol}, 5$ equiv.) and a magnetic stir bar. Upon addition of TFA the solution became yellow. The reaction was allowed to stir overnight forming sedimentation of NG that is no longer soluble in the organic solvents. Water was added to the reaction mixture and TFA and DCM were removed under reduced pressure. The aqueous solution was dialyzed against water for 2 days. Freeze drying yielded a white solid (90 mg, 100\% conversion).

\subsection{References}

1. Elsabahy, M.; Heo, G. S.; Lim, S.-M.; Sun, G.; Wooley, K. L., Polymeric nanostructures for imaging and therapy. Chemical reviews 2015, 115 (19), 1096711011.

2. Riehemann, K.; Schneider, S. W.; Luger, T. A.; Godin, B.; Ferrari, M.; Fuchs, H., Nanomedicine-challenge and perspectives. Angewandte Chemie International Edition 2009, 48 (5), 872-897.

3. Kelkar, S. S.; Reineke, T. M., Theranostics: combining imaging and therapy. Bioconjugate chemistry 2011, 22 (10), 1879-1903.

4. Thanh, N. T.; Green, L. A., Functionalisation of nanoparticles for biomedical applications. Nano Today 2010, 5 (3), 213-230.

5. Elsabahy, M.; Wooley, K. L., Design of polymeric nanoparticles for biomedical delivery applications. Chemical Society Reviews 2012, 41 (7), 2545-2561. 
6. Sharma, A.; Garg, T.; Aman, A.; Panchal, K.; Sharma, R.; Kumar, S.; Markandeywar, T., Nanogel-an advanced drug delivery tool: Current and future. Artificial cells, nanomedicine, and biotechnology 2016, 44 (1), 165-177.

7. Steinhilber, D.; Rossow, T.; Wedepohl, S.; Paulus, F.; Seiffert, S.; Haag, R., A microgel construction kit for bioorthogonal encapsulation and $\mathrm{pH}$-controlled release of living cells. Angewandte Chemie International Edition 2013, 52 (51), 13538-13543.

8. Li, Y.; Maciel, D.; Rodrigues, J.; Shi, X.; Tomas, H., Biodegradable polymer nanogels for drug/nucleic acid delivery. Chemical reviews 2015, 115 (16), 8564-8608.

9. Kabanov, A. V.; Vinogradov, S. V., Nanogels as pharmaceutical carriers: finite networks of infinite capabilities. Angew Chem Int Ed Engl 2009, 48 (30), 5418-29.

10. Molina, M.; Asadian-Birjand, M.; Balach, J.; Bergueiro, J.; Miceli, E.; Calderón, M., Stimuli-responsive nanogel composites and their application in nanomedicine. Chemical Society Reviews 2015, 44 (17), 6161-6186.

11. Fujioka-Kobayashi, M.; Ota, M. S.; Shimoda, A.; Nakahama, K.-i.; Akiyoshi, K.; Miyamoto, Y.; Iseki, S., Cholesteryl group-and acryloyl group-bearing pullulan nanogel to deliver BMP2 and FGF18 for bone tissue engineering. Biomaterials 2012, 33 (30), 7613-7620.

12. Xia, Y.; He, X.; Cao, M.; Chen, C.; Xu, H.; Pan, F.; Lu, J. R., Thermoresponsive microgel films for harvesting cells and cell sheets. Biomacromolecules 2013, 14 (10), 3615-3625.

13. Yallapu, M. M.; Jaggi, M.; Chauhan, S. C., Design and engineering of nanogels for cancer treatment. Drug discovery today 2011, 16 (9-10), 457-463.

14. Dorwal, D., Nanogels as novel and versatile pharmaceuticals. Int J Pharm Pharm Sci 2012, 4 (3), 67-74. 
15. Maya, S.; Sarmento, B.; Nair, A.; Rejinold, N. S.; Nair, S. V.; Jayakumar, R., Smart stimuli sensitive nanogels in cancer drug delivery and imaging: a review. Current pharmaceutical design 2013, 19 (41), 7203-7218.

16. Soni, G.; Yadav, K. S., Nanogels as potential nanomedicine carrier for treatment of cancer: A mini review of the state of the art. Saudi Pharmaceutical Journal 2016, $24(2), 133-139$.

17. Hasegawa, U.; Shin-ichiro, M. N.; Kaul, S. C.; Hirano, T.; Akiyoshi, K., Nanogelquantum dot hybrid nanoparticles for live cell imaging. Biochemical and biophysical research communications 2005, 331 (4), 917-921.

18. Wu, W.; Shen, J.; Banerjee, P.; Zhou, S., Core-shell hybrid nanogels for integration of optical temperature-sensing, targeted tumor cell imaging, and combined chemo-photothermal treatment. Biomaterials 2010, 31 (29), 7555-7566.

19. Zhao, C.; Chen, Q.; Patel, K.; Li, L.; Li, X.; Wang, Q.; Zhang, G.; Zheng, J., Synthesis and characterization of $\mathrm{pH}$-sensitive poly ( $\mathrm{N}$-2-hydroxyethyl acrylamide)-acrylic acid (poly (HEAA/AA)) nanogels with antifouling protection for controlled release. Soft Matter 2012, 8 (30), 7848-7857.

20. Bridges, A. W.; Singh, N.; Burns, K. L.; Babensee, J. E.; Lyon, L. A.; García, A. J., Reduced acute inflammatory responses to microgel conformal coatings. Biomaterials 2008, 29 (35), 4605-4615.

21. Kim, H.-J.; Zhang, K.; Moore, L.; Ho, D., Diamond nanogel-embedded contact lenses mediate lysozyme-dependent therapeutic release. ACS nano 2014, 8 (3), 2998-3005.

22. Wu, W.; Shen, J.; Li, Y.; Zhu, H.; Banerjee, P.; Zhou, S., Specific glucose-to-SPR signal transduction at physiological $\mathrm{pH}$ by molecularly imprinted responsive hybrid microgels. Biomaterials 2012, 33 (29), 7115-7125. 
23. Wu, W.; Mitra, N.; Yan, E. C.; Zhou, S., Multifunctional hybrid nanogel for integration of optical glucose sensing and self-regulated insulin release at physiological pH. Acs Nano 2010, 4 (8), 4831-4839.

24. Li, C.; Liu, S., Responsive nanogel-based dual fluorescent sensors for temperature and $\mathrm{Hg} 2+$ ions with enhanced detection sensitivity. Journal of Materials Chemistry 2010, 20 (47), 10716-10723.

25. Zhu, H.; Li, Y.; Qiu, R.; Shi, L.; Wu, W.; Zhou, S., Responsive fluorescent Bi2O3@ PVA hybrid nanogels for temperature-sensing, dual-modal imaging, and drug delivery. Biomaterials 2012, 33 (10), 3058-3069.

26. Wu, W.; Shen, J.; Gai, Z.; Hong, K.; Banerjee, P.; Zhou, S., Multi-functional coreshell hybrid nanogels for $\mathrm{pH}$-dependent magnetic manipulation, fluorescent $\mathrm{pH}$ sensing, and drug delivery. Biomaterials 2011, 32 (36), 9876-9887.

27. Peng, H. S.; Stolwijk, J. A.; Sun, L. N.; Wegener, J.; Wolfbeis, O. S., A nanogel for ratiometric fluorescent sensing of intracellular $\mathrm{pH}$ values. Angewandte Chemie 2010, 122 (25), 4342-4345.

28. Wu, W.; Aiello, M.; Zhou, T.; Berliner, A.; Banerjee, P.; Zhou, S., In-situ immobilization of quantum dots in polysaccharide-based nanogels for integration of optical pH-sensing, tumor cell imaging, and drug delivery. Biomaterials 2010, 31 (11), 3023-3031.

29. Hoare, T.; Young, S.; Lawlor, M. W.; Kohane, D. S., Thermoresponsive nanogels for prolonged duration local anesthesia. Acta biomaterialia 2012, 8 (10), 3596-3605.

30. Oh, J. K.; Drumright, R.; Siegwart, D. J.; Matyjaszewski, K., The development of microgels/nanogels for drug delivery applications. Progress in Polymer Science 2008, 33 (4), 448-477.

31. Nayak, S.; Lyon, L. A., Soft nanotechnology with soft nanoparticles. Angewandte chemie international edition 2005, 44 (47), 7686-7708. 
32. Schubert, S.; Delaney Jr, J. T.; Schubert, U. S., Nanoprecipitation and nanoformulation of polymers: from history to powerful possibilities beyond poly (lactic acid). Soft Matter 2011, 7 (5), 1581-1588.

33. Lepeltier, E.; Bourgaux, C.; Couvreur, P., Nanoprecipitation and the "Ouzo effect": Application to drug delivery devices. Advanced drug delivery reviews 2014, 71, 8697.

34. Rolland, J. P.; Maynor, B. W.; Euliss, L. E.; Exner, A. E.; Denison, G. M.; DeSimone, J. M., Direct fabrication and harvesting of monodisperse, shape-specific nanobiomaterials. Journal of the American Chemical Society 2005, 127 (28), 10096-10100.

35. Venturoli, D.; Rippe, B., Ficoll and dextran vs. globular proteins as probes for testing glomerular permselectivity: effects of molecular size, shape, charge, and deformability. American Journal of Physiology-Renal Physiology 2005, 288 (4), F605-F613.

36. Ogawara, K.-I.; Yoshida, M.; Furumoto, K.; Takakura, Y.; Hashida, M.; Higaki, K.; Kimura, T., Uptake by hepatocytes and biliary excretion of intravenously administered polystyrene microspheres in rats. Journal of drug targeting 1999, 7 (3), 213-221.

37. Grazon, C.; Rieger, J.; Sanson, N.; Charleux, B., Study of poly (N, Ndiethylacrylamide) nanogel formation by aqueous dispersion polymerization of $\mathrm{N}$, $\mathrm{N}$-diethylacrylamide in the presence of poly (ethylene oxide)-b-poly $(\mathrm{N}, \mathrm{N}$ dimethylacrylamide) amphiphilic macromolecular RAFT agents. Soft Matter 2011, $7(7), 3482-3490$.

38. Ding, P.; Huang, J.; Wei, C.; Liu, W.; Zhou, W.; Wang, J.; Wang, M.; Guo, X.; Cohen Stuart, M. A.; Wang, J., Efficient and Generic Preparation of Diverse Polyelectrolyte Nanogels by Electrostatic Assembly Directed Polymerization. CCS Chemistry 2020, 2 (6), 1016-1025. 
39. Ryu, J.-H.; Chacko, R. T.; Jiwpanich, S.; Bickerton, S.; Babu, R. P.; Thayumanavan, S., Self-cross-linked polymer nanogels: a versatile nanoscopic drug delivery platform. Journal of the American Chemical Society 2010, 132 (48), 17227-17235.

40. Li, R.-Q.; Wu, W.; Song, H.-Q.; Ren, Y.; Yang, M.; Li, J.; Xu, F.-J., Well-defined reducible cationic nanogels based on functionalized low-molecular-weight PGMA for effective pDNA and siRNA delivery. Acta biomaterialia 2016, 41, 282-292.

41. Oh, J. K.; Tang, C.; Gao, H.; Tsarevsky, N. V.; Matyjaszewski, K., Inverse miniemulsion ATRP: a new method for synthesis and functionalization of welldefined water-soluble/cross-linked polymeric particles. Journal of the American Chemical Society 2006, 128 (16), 5578-5584.

42. Hellmund, M.; Zhou, H.; Samsonova, O.; Welker, P.; Kissel, T.; Haag, R., Functionalized polyglycerol amine nanogels as nanocarriers for DNA. Macromolecular bioscience 2014, 14 (9), 1215-1221.

43. Sütekin, S. D.; Güven, O., Application of radiation for the synthesis of poly (n-vinyl pyrrolidone) nanogels with controlled sizes from aqueous solutions. Applied Radiation and Isotopes 2019, 145, 161-169.

44. van der Ende, A. E.; Kravitz, E. J.; Harth, E., Approach to formation of multifunctional polyester particles in controlled nanoscopic dimensions. J Am Chem Soc 2008, 130, 8706-8713.

45. Gao, Y.; Newland, B.; Zhou, D.; Matyjaszewski, K.; Wang, W., Controlled Polymerization of Multivinyl Monomers: Formation of Cyclized/Knotted SingleChain Polymer Architectures. Angewandte Chemie International Edition 2017, 56 (2), 450-460.

46. Boyer, C.; Bulmus, V.; Davis, T. P.; Ladmiral, V.; Liu, J.; Perrier, S., Bioapplications of RAFT polymerization. Chemical reviews 2009, 109 (11), 5402-5436. 
47. Picos-Corrales, L. A.; Licea-Claveríe, A.; Arndt, K. F., Core-shell nanogels by RAFT crosslinking polymerization: Synthesis and characterization. Journal of Polymer Science Part A: Polymer Chemistry 2012, 50 (20), 4277-4287.

48. Rosselgong, J.; Armes, S. P.; Barton, W.; Price, D., Synthesis of highly branched methacrylic copolymers: observation of near-ideal behavior using RAFT polymerization. Macromolecules 2009, 42 (16), 5919-5924.

49. Nuhn, L.; Hirsch, M.; Krieg, B.; Koynov, K.; Fischer, K.; Schmidt, M.; Helm, M.; Zentel, R., Cationic nanohydrogel particles as potential siRNA carriers for cellular delivery. Acs Nano 2012, 6 (3), 2198-2214.

50. Nuhn, L.; Gietzen, S.; Mohr, K.; Fischer, K.; Toh, K.; Miyata, K.; Matsumoto, Y.; Kataoka, K.; Schmidt, M.; Zentel, R., Aggregation behavior of cationic nanohydrogel particles in human blood serum. Biomacromolecules 2014, 15 (4), 1526-1533.

51. Nuhn, L.; Tomcin, S.; Miyata, K.; Mailänder, V.; Landfester, K.; Kataoka, K.; Zentel, R., Size-dependent knockdown potential of siRNA-loaded cationic nanohydrogel particles. Biomacromolecules 2014, 15 (11), 4111-4121.

52. Alexis, F.; Pridgen, E.; Molnar, L. K.; Farokhzad, O. C., Factors affecting the clearance and biodistribution of polymeric nanoparticles. Molecular pharmaceutics 2008, 5 (4), 505-515.

53. Li, Y.; Beija, M.; Laurent, S.; Elst, L. v.; Muller, R. N.; Duong, H. T.; Lowe, A. B.; Davis, T. P.; Boyer, C., Macromolecular ligands for gadolinium MRI contrast agents. Macromolecules 2012, 45 (10), 4196-4204.

54. Hernández-Adame, L.; Angulo, C.; García-Silva, I.; Palestino, G.; Rosales-Mendoza, S., An overview of nanogel-based vaccines. Expert review of vaccines 2019, 18 (9), 951-968. 
55. Kolb, H. C.; Finn, M. G.; Sharpless, K. B., Click Chemistry: Diverse Chemical Function from a Few Good Reactions. Angewandte Chemie International Edition 2001, 40 (11), 2004-2021.

56. Bruice, P. Y., Organic Chemistry (7th ed.). Pearson: London, 2014.

57. Gao, H.; Matyjaszewski, K., Synthesis of functional polymers with controlled architecture by CRP of monomers in the presence of cross-linkers: From stars to gels. Progress in Polymer Science 2009, 34 (4), 317-350.

58. Thelu, H. V. P.; Atchimnaidu, S.; Perumal, D.; Harikrishnan, K. S.; Vijayan, S.; Varghese, R., Self-assembly of an aptamer-decorated, DNA-protein hybrid nanogel: a biocompatible nanocarrier for targeted cancer therapy. ACS Applied Bio Materials 2019, 2 (12), 5227-5234.

59. Wu, L.; Zhang, J.; Watanabe, W., Physical and chemical stability of drug nanoparticles. Advanced drug delivery reviews 2011, 63 (6), 456-469.

60. Kolb, H. C.; Finn, M.; Sharpless, K. B., Click chemistry: diverse chemical function from a few good reactions. Angewandte Chemie International Edition 2001, 40 (11), 2004-2021.

61. Weaver, J.; Bannister, I.; Robinson, K.; Bories-Azeau, X.; Armes, S.; Smallridge, M.; McKenna, P., Stimulus-responsive water-soluble polymers based on 2hydroxyethyl methacrylate. Macromolecules 2004, 37 (7), 2395-2403.

62. Muzammil, E. M.; Khan, A.; Stuparu, M. C., Post-polymerization modification reactions of poly (glycidyl methacrylate) s. RSC Advances 2017, 7 (88), 5587455884.

63. Gadwal, I.; Stuparu, M. C.; Khan, A., Homopolymer bifunctionalization through sequential thiol-epoxy and esterification reactions: an optimization, quantification, and structural elucidation study. Polymer Chemistry 2015, 6 (8), 1393-1404. 
64. Hwang, J.; Choe, Y.; Bang, J.; Khan, A., Scalable ambient synthesis of water-soluble poly ( $\beta$-hydroxythio-ether) s. Journal of Polymer Science Part A: Polymer Chemistry 2017, 55 (20), 3381-3386.

65. Hwang, J.; Lee, D. G.; Yeo, H.; Rao, J.; Zhu, Z.; Shin, J.; Jeong, K.; Kim, S.; Jung, H. W.; Khan, A., Proton Transfer Hydrogels: Versatility and Applications. Journal of the American Chemical Society 2018.

66. Stuparu, M. C.; Khan, A., Thiol-epoxy "click" chemistry: Application in preparation and postpolymerization modification of polymers. Journal of Polymer Science Part A: Polymer Chemistry 2016, 54 (19), 3057-3070.

67. Yang, X.; Chai, M.; Zhu, Y.; Yang, W.; Xu, F., Facilitation of gene transfection with well-defined degradable comb-shaped poly (glycidyl methacrylate) derivative vectors. Bioconjugate chemistry 2012, 23 (3), 618-626.

68. Li, R.; Niu, Y.; Zhao, N.; Yu, B.; Mao, C.; Xu, F., Series of new $\beta$-cyclodextrin-cored starlike carriers for gene delivery. ACS applied materials \& interfaces 2014, 6 (6), 3969-3978.

69. Li, R.; Hu, Y.; Yu, B.; Zhao, N.; Xu, F., Bioreducible comb-shaped conjugates composed of secondary amine and hydroxyl group-containing backbones and disulfide-linked side chains with tertiary amine groups for facilitating gene delivery. Bioconjugate chemistry 2014, 25 (1), 155-164.

70. Yang, X.-C.; Niu, Y.-L.; Zhao, N.-N.; Mao, C.; Xu, F.-J., A biocleavable pullulan-based vector via ATRP for liver cell-targeting gene delivery. Biomaterials 2014, 35 (12), 3873-3884.

71. Dou, X.; Chai, M.; Zhu, Y.; Yang, W.; Xu, F., Aminated poly (glycidyl methacrylate) s for constructing efficient gene carriers. ACS applied materials \& interfaces 2013, $5(8), 3212-3218$. 
72. Gao, H.; Elsabahy, M.; Giger, E. V.; Li, D.; Prud'homme, R. E.; Leroux, J.-C., Aminated linear and star-shape poly (glycerol methacrylate) s: synthesis and selfassembling properties. Biomacromolecules 2010, 11 (4), 889-895.

73. Ma, M.; Li, F.; Chen, F. j.; Cheng, S. x.; Zhuo, R. x., Poly (ethylene glycol)-block-Poly (glycidyl methacrylate) with Oligoamine Side Chains as Efficient Gene Vectors. Macromolecular bioscience 2010, 10 (2), 183-191.

74. Edmondson, S.; Huck, W. T., Controlled growth and subsequent chemical modification of poly (glycidyl methacrylate) brushes on silicon wafers. Journal of Materials Chemistry 2004, 14 (4), 730-734.

75. Kabanov, A. V.; Vinogradov, S. V., Nanogels as pharmaceutical carriers: finite networks of infinite capabilities. Angewandte Chemie International Edition 2009, 48 (30), 5418-5429.

76. Tsarevsky, N. V.; Bencherif, S. A.; Matyjaszewski, K., Graft copolymers by a combination of ATRP and two different consecutive click reactions. Macromolecules 2007, 40 (13), 4439-4445.

77. Chini, M.; Crotti, P.; Macchia, F., Efficient metal salt catalyzed azidolysis of epoxides with sodium azide in acetonitrile. Tetrahedron letters 1990, 31 (39), 5641-5644.

78. Mauri, E.; Moroni, I.; Magagnin, L.; Masi, M.; Sacchetti, A.; Rossi, F., Comparison between two different click strategies to synthesize fluorescent nanogels for therapeutic applications. Reactive and Functional Polymers 2016, 105, 35-44.

79. Bräse, S.; Gil, C.; Knepper, K.; Zimmermann, V., Organic azides: an exploding diversity of a unique class of compounds. Angewandte Chemie International Edition 2005, 44 (33), 5188-5240.

80. Ferguson, C. J.; Hughes, R. J.; Nguyen, D.; Pham, B. T.; Gilbert, R. G.; Serelis, A. K.; Such, C. H.; Hawkett, B. S., Ab initio emulsion polymerization by RAFT-controlled self-assembly. Macromolecules 2005, 38 (6), 2191-2204. 
Chapter 3

81. Nicosia, C.; Krabbenborg, S. O.; Reinhoudt, D. N.; Huskens, J., In situ fluorimetric detection of micrometer-scale $\mathrm{pH}$ gradients at the solid/liquid interface. Supramolecular chemistry 2013, 25 (9-11), 756-766. 


\subsection{Supplementary data}

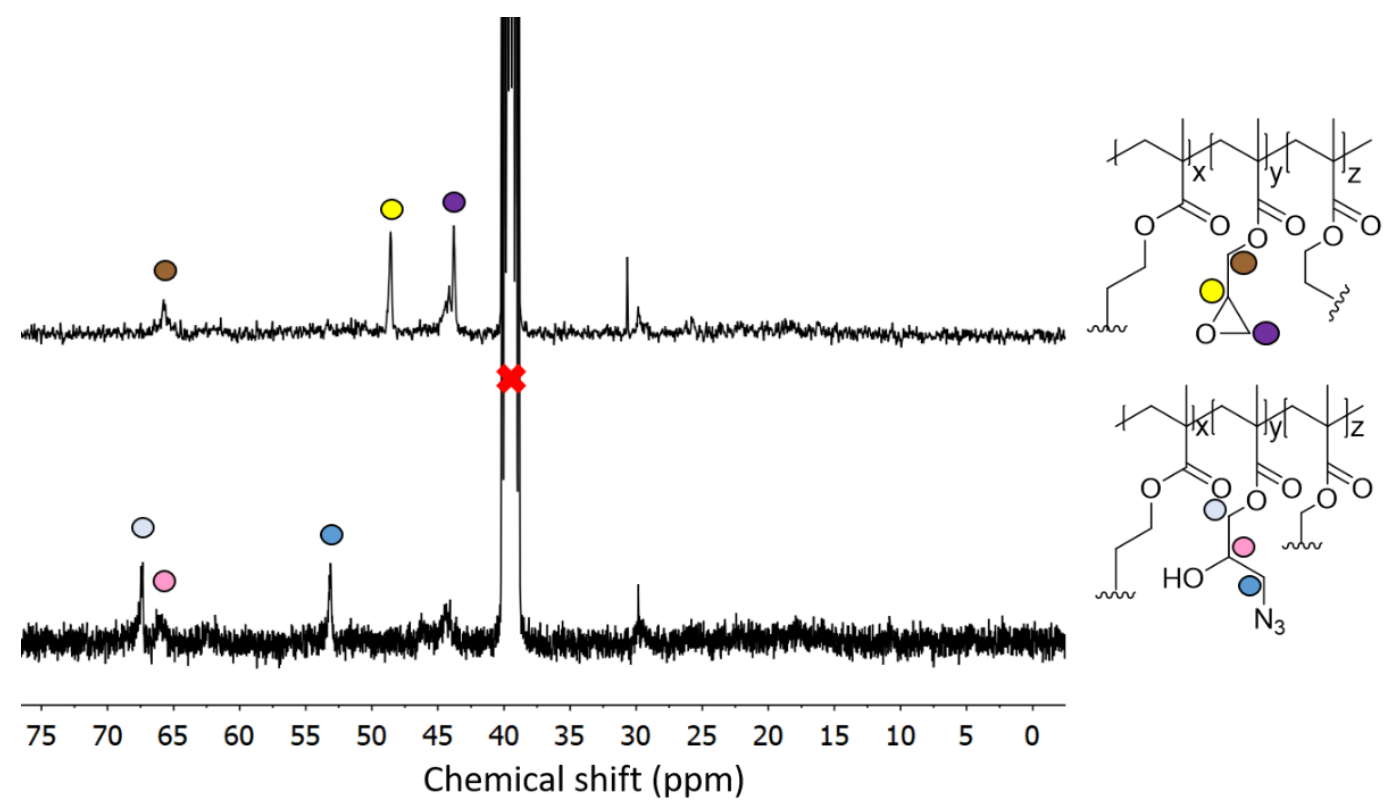

Figure S1. Zoomed in ${ }^{13} \mathrm{C}$ NMR spectrum of GMA-EGDMA-N $\mathbf{N}_{3}$ (bottom) in DMSO- $\mathrm{d}_{6}$ indicates the presence of $\mathrm{C}-\mathrm{N}_{3}$ bonds at $\delta=53 \mathrm{ppm}$.
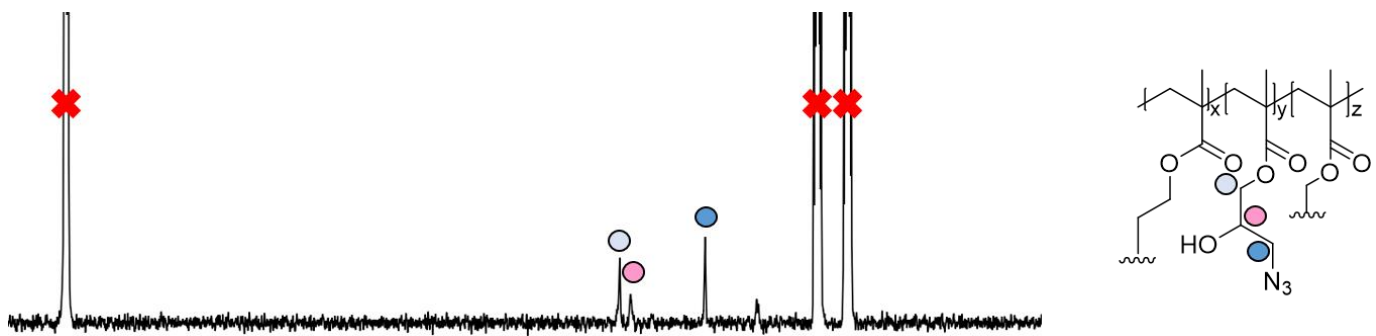

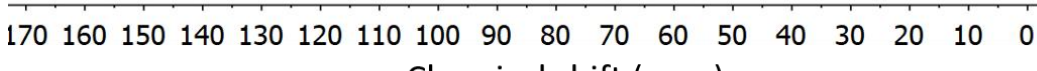

Chemical shift (ppm)

Figure S2. ${ }^{13} \mathrm{C}$ NMR spectrum of GMA-EGDMA-N $\mathbf{N}_{3}$ in DMF- $\mathrm{d}_{7}$ indicates the presence of $\mathrm{C}-\mathrm{N}_{3}$ bonds at $\delta=55$ ppm. 
Chapter 3

132 | $P$ a g e 


\section{Chapter 4}

\section{Post-polymerization functionalization of the epoxy moiety to sulfonium nanogels}

Nano-sized hydrogel particles, also known as nanogels, are crosslinked polymeric nanoparticles that are promising materials for biomedical applications. By incorporating positively charged sulfur groups, also known as sulfonium groups, these materials could be used as cationic carriers for gene delivery. In this chapter glycidyl methacrylateethylene glycol dimethacrylate (GMA-EGDMA) nanogels are synthesized as a platform for subsequent functionalization. Post-polymerization modifications of these nanogels was achieved by ring-opening reactions of the GMA epoxy moieties. Ring-opening reactions with diethyl sulfide were carried out to install permanent cationic sulfonium groups on the nanogels, yielding readily water-soluble nanogels with a zeta potential of $\zeta=+40 \mathrm{mV}$ at neutral $\mathrm{pH}$ and a mean diameter of $\mathrm{D}=29 \mathrm{~nm}$ as determined by transmission electron microscopy (TEM). The degree of functionalization with sulfonium groups was found to be tunable. Particles with a low degree of functionalization require a more hydrophilic nanogel structure to yield solubility in water at ambient temperature and a minimum concentration of $2 \mathrm{mg} / \mathrm{mL}$.

The work presented here serves as a foundation for the synthesis of sulfoniumfunctionalized nanogels with tunable particle size and charge density.

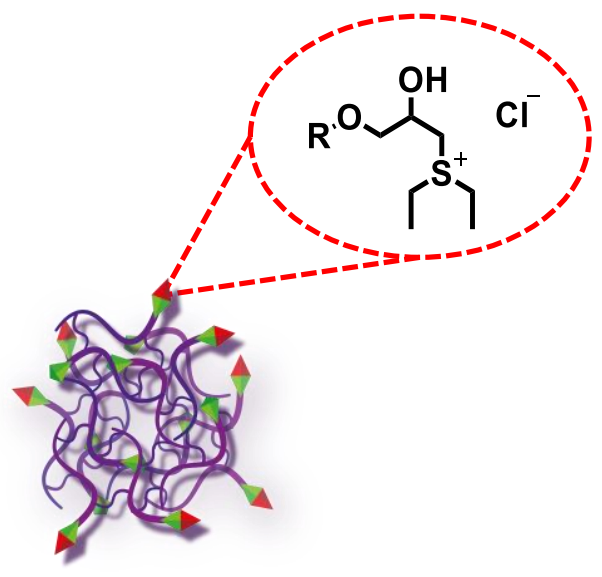




\subsection{Introduction}

\subsubsection{Gene therapy}

Gene therapy is a promising therapeutic strategy applicable for treatment of inherited or acquired disorders, such as AIDS ${ }^{1-2}$ and cancer $^{3-4}$ by using exogenous genetic material as medicine, thereby influencing protein expression in cells. ${ }^{5-6}$ There are three general approaches to gene therapy, the first of which involves replacing a dysfunctional gene by a healthy copy (DNA or mRNA). Another approach is introducing new or missing genes for the expression of a therapeutic protein. The third approach involves silencing of a mutated gene by interfering with its expression after transcription. For example, by introducing complementary short strands of non-coding RNA, also known as microRNA, mRNA translation can be downregulated, reducing gene expression. ${ }^{7-8}$ However, therapeutic use of DNA or RNA comes with challenges; simply injecting nucleic acids intravenously leads to enzymatic degradation by nucleases, renal clearance, and clearance by the mononuclear phagocyte system. ${ }^{9-11}$ Therefore, delivery systems, also known as vectors, are needed to safely and effectively deliver therapeutic genes in vivo.

\subsubsection{Gene delivery vectors in nanomedicine}

An ideal nanocarrier should protect the genetic material from enzymatic degradation in physiological fluids and removal by macrophages and the kidneys. As cellular membranes are negatively charged, the nanocarrier should also neutralize or shield the negative charges of the nucleic acids to prevent electrostatic repulsion. Ideally, the nanocarrier should allow for easy functionalization with targeting ligands for specific uptake. Also, the carrier should allow for precise control over the amount of gene expression and should regulate the release of genetic material. Furthermore, the nanocarrier should be 
biocompatible to prevent side effects and be easy to produce on a commercial scale at a reasonable cost. ${ }^{12-13}$ Satisfying these criteria requires the nanocarrier to have tunable properties such as size, shape, surface charge and hydrophilicity to influence its interactions with biological systems. ${ }^{14}$ For example, nanoparticles with a diameter smaller than $10 \mathrm{~nm}$ are subject to renal clearance from the blood stream. ${ }^{15}$ Conversely, large particles with a diameter exceeding roughly $200 \mathrm{~nm}$ are cleared by the mononuclear phagocyte system, accumulating in the liver and spleen. ${ }^{16-17}$ Thus, the optimal size for a nanocarrier (including its cargo) should be between 10 and $200 \mathrm{~nm}$ for prolonged circulation and optimal biodistribution. Both size and surface charge influence cellular uptake. Positively charged particles are known to adsorb onto the negatively charged cell membrane, leading to an improvement in uptake efficiency compared to neutral or negatively charged particles. ${ }^{18}$ After adsorption to the outer cell membrane, these particles are internalized by adsorptive endocytosis. ${ }^{19-20}$ Particles smaller than $100 \mathrm{~nm}$ can generally be encapsulated in endocytic vesicles, allowing for cellular entry through endocytosis. ${ }^{21}$ Therefore, the ideal particle size is larger than $10 \mathrm{~nm}$ - to prevent renal clearance, and smaller than $100 \mathrm{~nm}$ to stimulate endocytosis. ${ }^{20}$

Gene delivery vectors can generally be classified into three groups: viral vectors, physical vectors, and synthetic (chemical) vectors. Whereas viral and synthetic vectors are based on the encapsulation or complexation of nucleic acids into nano-sized carrier, physical vectors rely on forcing nucleic acids across the cell membrane by an external stimulus. Although viral vectors such as the lentivirus ${ }^{22-23}$ and the adenovirus ${ }^{24}$ generally offer higher transfection efficiencies, these systems often cause an immune response in vivo, in addition to their potential mutagenic effects. ${ }^{6,12,25-26}$ The high cost of production is also not favorable. As such, synthetic gene delivery systems pose safer and cheaper alternatives. Synthetic vectors can be inorganic, lipid-based, and polymeric. Lipid-based vectors employ amphiphilic lipids to encapsulate nucleic acids in liposomes or micelles for 
therapeutic use. ${ }^{27-30}$ Although several limitations are associated with lipid-based vectors, such as toxicity, premature payload release ${ }^{31-34}$ and poor stability ${ }^{35-37}$, these vectors are among the most widely used non-viral vectors. ${ }^{38-44}$ Polymeric vectors are based on a polymer/nucleic acid complex (polyplex), formed by electrostatic interactions between a positively charged polymer and negatively charged nucleic acids. Polyethylenimine (PEI), is one of the most well-known examples of synthetic polymeric vectors. ${ }^{45-46} \mathrm{PEI}$ has been used for gene transfection in vivo, in linear or branched forms and with varying molecular weights, yielding varying degrees of transfection efficiency and cytotoxicity. ${ }^{47-48}$ Moreover, the buffering capacity of the nitrogen atoms, able to be protonated in PEI, has been proposed to aid in endosomal escape due to an increase in osmotic pressure upon acidification of endosomes - the so called "proton sponge effect". ${ }^{46,49-50}$ In addition to linear or branched polymers, many nano-sized polymer architectures have been utilized for gene delivery, such as dendrimers ${ }^{51-52}$ and polymersomes ${ }^{53}$.

\subsubsection{The synthesis of nanogel carriers via controlled crosslinking polymerization}

Other polymer nanostructures include nanogels which are crosslinked three-dimensional polymer networks able to be highly biocompatible with high (therapeutic) cargo loading. ${ }^{54}$ Nanogels typically have a network-like structure with inter- as well as intramolecular crosslinks. ${ }^{55-56}$ Nanogels are generally prepared by (inverse) microemulsion methods that consist of an oil-in-water strategy producing hydrophobic nanogels or hydrophilic nanogels that require multiple tedious steps to purify. ${ }^{57-59}$

Nanogels can be synthesized by controlled crosslinking polymerization (CCP) of monomers with multi-functional co-monomers, relying on simultaneous polymer growth and crosslinking in a homogenous reaction mixture. ${ }^{60}$ Nanogels synthesized via living polymerization have a very similar degree of polymerization across all individual polymer 
chains, leading to a low polydispersity index. This allows for the synthesis of precisely designed polymer nanostructures with a high degree of control over molecular mass, making it especially suitable for the synthesis of small nanogels $(<100 \mathrm{~nm}) \cdot{ }^{54,61-62}$ Nanogels have been shown to be high functioning delivery vehicles for many biomedical applications including gene delivery. ${ }^{63-64}$ Nanogels are highly sensitive to stimuli and require minor changes in physiological conditions to exert a response, as opposed to conventional drug delivery platforms. ${ }^{65-67}$

\subsubsection{Post-polymerization modifications of epoxide-containing polymers to sulfonium polymers}

Cationic polymers can be synthesized by polymerizing cationic monomers ${ }^{68}$ or by postpolymerization modifications that introduce positive charge. The advantage of the second method is that one scaffold can be used for the synthesis of a family of materials with similar macrostructure (e.g. degree of crosslinking, particle size, degree of polymerization) but different functionality. ${ }^{69}$ Positive charge can be installed on polymer pendent groups by alkylation of a thioether with an alkyl halide, forming a sulfonium group. ${ }^{70-71}$ Kramer et al. ${ }^{71}$ utilized this strategy for the post-polymerization functionalization of poly-(Lmethionine) by means of a "click" type reaction as shown in Scheme 1. 


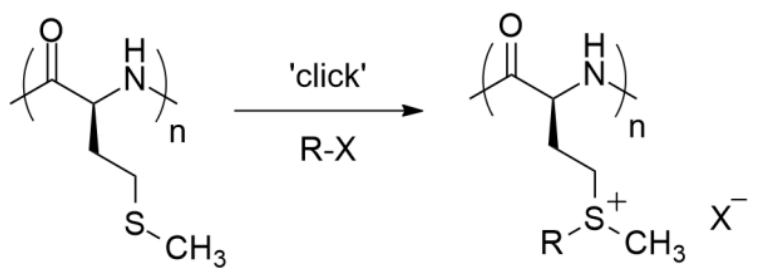

$$
\begin{gathered}
R=\text { functional alkyl group } \\
X=\text { halide }
\end{gathered}
$$

Scheme 1. Post-polymerization modification of thioether moieties using alkyl halides ( $R-X)$ in a "click" type reaction to functionalize poly-(L-methionine), as demonstrated by Kramer et al. $^{71}$.

Ethers $\left(\mathrm{R}_{1}-\mathrm{O}-\mathrm{R}_{2}\right)$ do not react with alkyl halides due to their poor nucleophilicity and inability to accommodate positive charge. Thioethers $\left(R_{1}-S-R_{2}\right)$ on the contrary are relatively good nucleophiles due to the large atomic radius of sulfur, which yields polarizable electron clouds. ${ }^{72}$ Likewise, polymers employing thioether moieties were shown to react with epoxides under acidic conditions, forming charged sulfonium groups in a single step. ${ }^{73-74}$
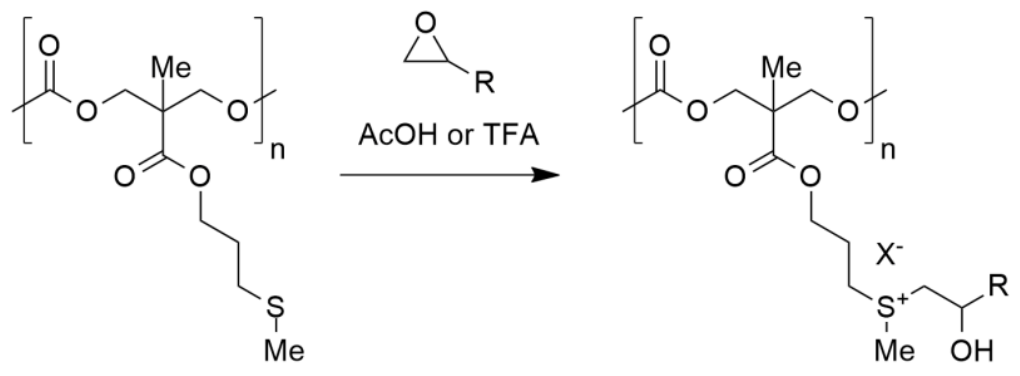

$X=O A c$, TFA

Scheme 2. Post-polymerization modification employing thioether-epoxide chemistry, as shown by Park et al. ${ }^{73}$ 
In this chapter we synthesize sulfonium nanogels from two glycidyl methacrylate nanogel precursors - achieving various degrees of epoxide conversions. The remaining epoxides undergo a second ring opening reaction with TFA, thioglycerol or thiol-PEG to quench all epoxides and increase hydrophilicity.

\subsection{Results and Discussion}

\subsubsection{GMA-EGDMA nanogel functionalizations}

GMA-EGDMA nanogel was synthesized as described in Chapter 3. Once a desirable monomer conversion was achieved (30\%), a large excess of AIBN was added to the reaction mixture to quench the remaining methacrylates. Dynamic Light Scattering (DLS) measurements gave a Z-average hydrodynamic diameter of $60 \mathrm{~nm}$ (in DCM) and analysis by Transmission Electron Microscopy (TEM) resulted in a similar particle diameter of 56 $\mathrm{nm}$.

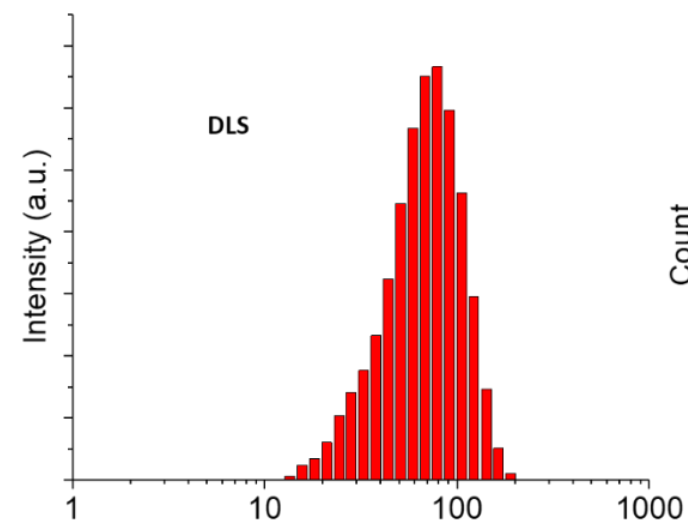

Hydrodynamic diameter $(\mathrm{nm})$

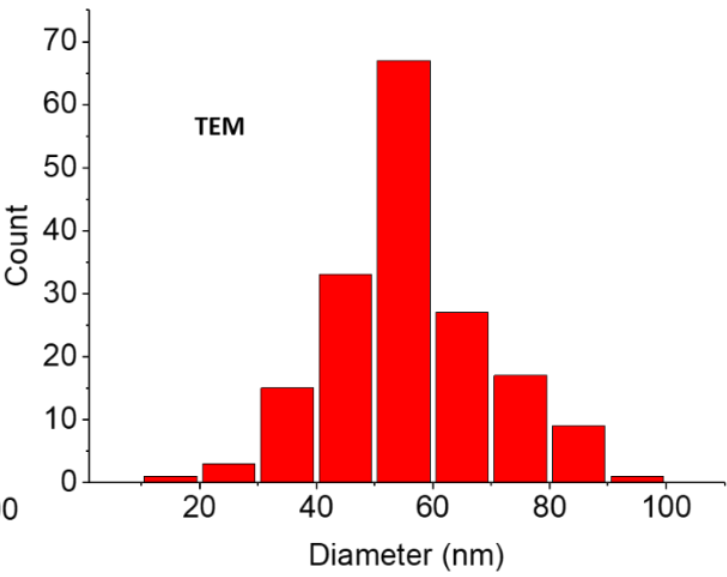

Figure 1. GMA-EGDMA nanogel at $30 \%$ monomer conversion was analyzed by DLS and TEM displaying unimodal distribution for both techniques. 
The next step towards the formation of a gene carrier is the installation of charge. Plasmid DNA that is utilized to traffic foreign genes into the human body has a negative charge. As such it is desired to have the gene vector be positively charged to facilitate interaction and ensure proper conjugation. Utilizing the method first published by Park ${ }^{73}$ - GMAEGDMA nanogel was functionalized with diethyl sulfide in a ring-opening reaction to yield a sulfonium-functionalized nanogel.

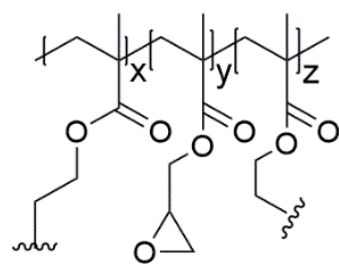

GMA-EGDMA
1. 13 eq. diethyl sulfide acetone/AcOH, 1:1 $\mathrm{rt}, 24 \mathrm{~h}$

2. Dialysis against $0.1 \mathrm{M} \mathrm{NaCl}$

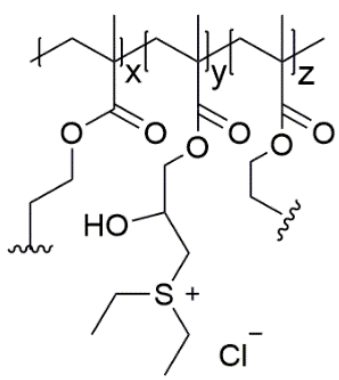

GMA-EGDMA-S

Scheme 3. The synthesis of sulfonium-functionalized GMA-EGDMA-S+ was followed by dialysis against $0.1 \mathrm{M}$ $\mathrm{NaCl}$ to exchange the acetate counter ion for a chlorine counter ion.

Figure 2 displays the ${ }^{1} \mathrm{H}$ NMR spectrum of GMA-EGDMA-S ${ }^{+}$at full epoxide conversion. Characteristic signals of the sulfonium group are labeled at $\delta=1.4 \mathrm{ppm}$ and $\delta=3.5 \mathrm{ppm}$. These signals are shifted upfield compared to uncharged thioether, as expected. Full conversion of epoxides is assessed by the absence of epoxide signals at $\delta=2.6 \mathrm{ppm}, \delta=$ $2.8 \mathrm{ppm}$ and $\delta=3.2 \mathrm{ppm}$. 


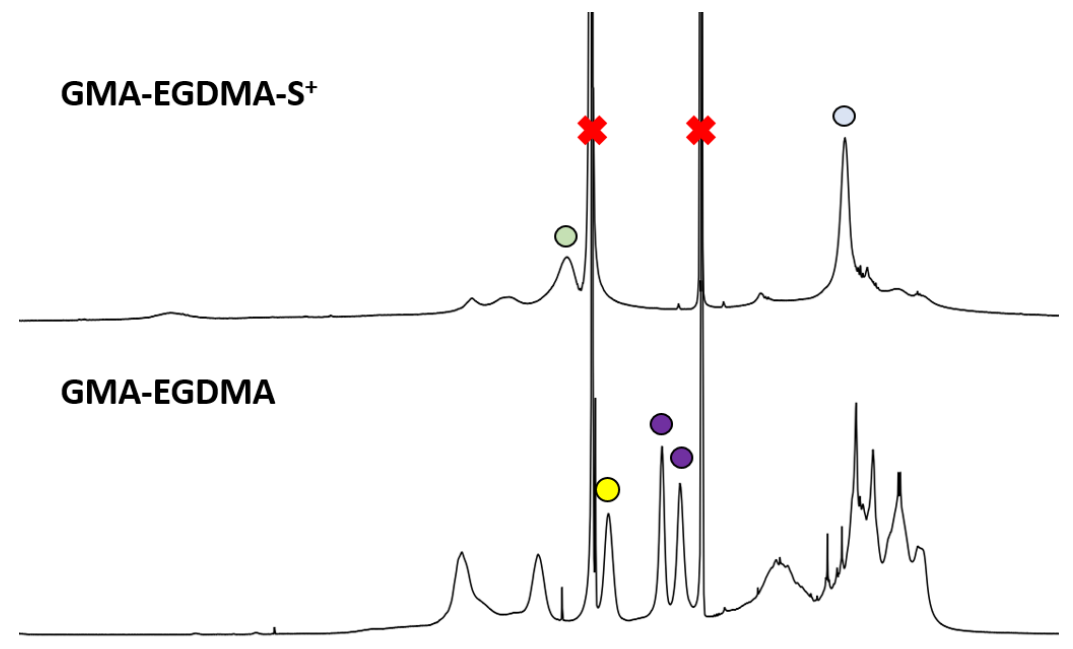

$\begin{array}{llllllllllllllll}7.5 & 7.0 & 6.5 & 6.0 & 5.5 & 5.0 & 4.5 & 4.0 & 3.5 & 3.0 & 2.5 & 2.0 & 1.5 & 1.0 & 0.5 & 0.0\end{array}$
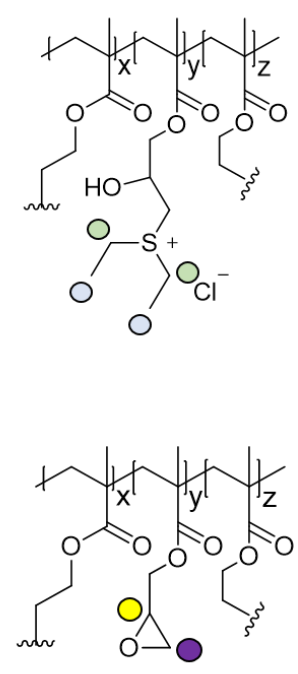

Chemical shift (ppm)

Figure 2. ${ }^{1} \mathrm{H}$ NMR spectrum of GMA-EGDMA-S ${ }^{+}$, with characteristic signals of the sulfonium group at $\delta=1.4$ ppm labelled blue and at $\delta=3.5$ ppm labelled green.

The zeta potential of GMA-EGDMA-S ${ }^{+}$was determined to be $+40 \mathrm{mV}$ at neutral $\mathrm{pH}$ (in 5 $\mathrm{mM} \mathrm{KCl}$ ), meaning a high surface charge. This is reflected back in the hydrodynamic diameter of GMA-EGDMA-S ${ }^{+}$compared to GMA-EGDMA. DLS measurements indicated a size increase from $60 \mathrm{~nm}$ to $95 \mathrm{~nm}$, likely the result of the positive charges repelling each other. 


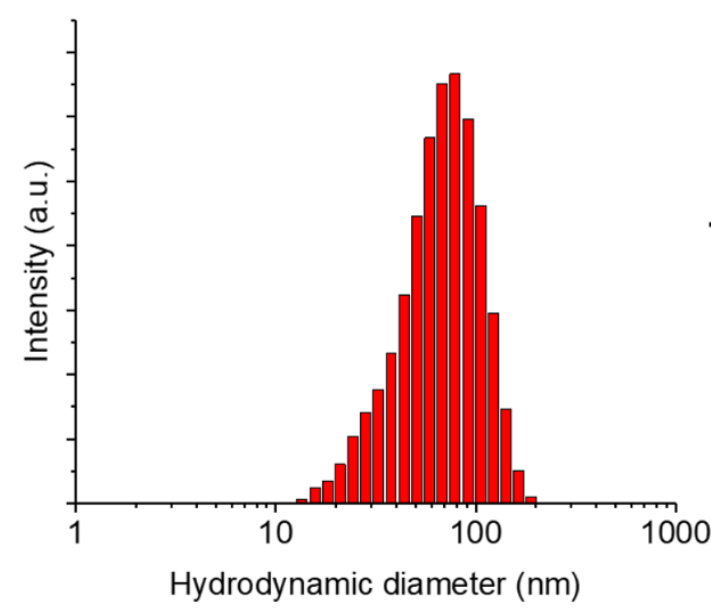

GMA-EGDMA

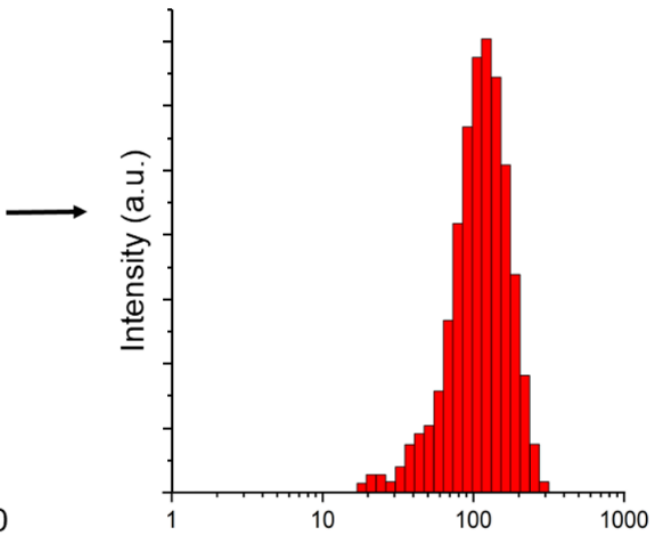

Hydrodynamic diameter $(\mathrm{nm})$

GMA-EGDMA-S+

Figure 3. The hydrodynamic diameters of GMA-EGDMA-S+ (in $5 \mathrm{mM} \mathrm{KCl)} \mathrm{and} \mathrm{GMA-EGDMA} \mathrm{(in} \mathrm{DCM)} \mathrm{were}$ measured by means of DLS indicating a semi bimodal size distribution. This increase in size is likely the result of same charges repelling each other as well as a difference in solvents.

The dynamic nature of these nanogels was further illustrated by TEM analysis. A mean particle diameter of $29 \mathrm{~nm}$ was measured, which is significantly lower than described above. This is expected as the nanogel is soft and well-solvated during DLS measurements as opposed to high vacuum during TEM measurements. 


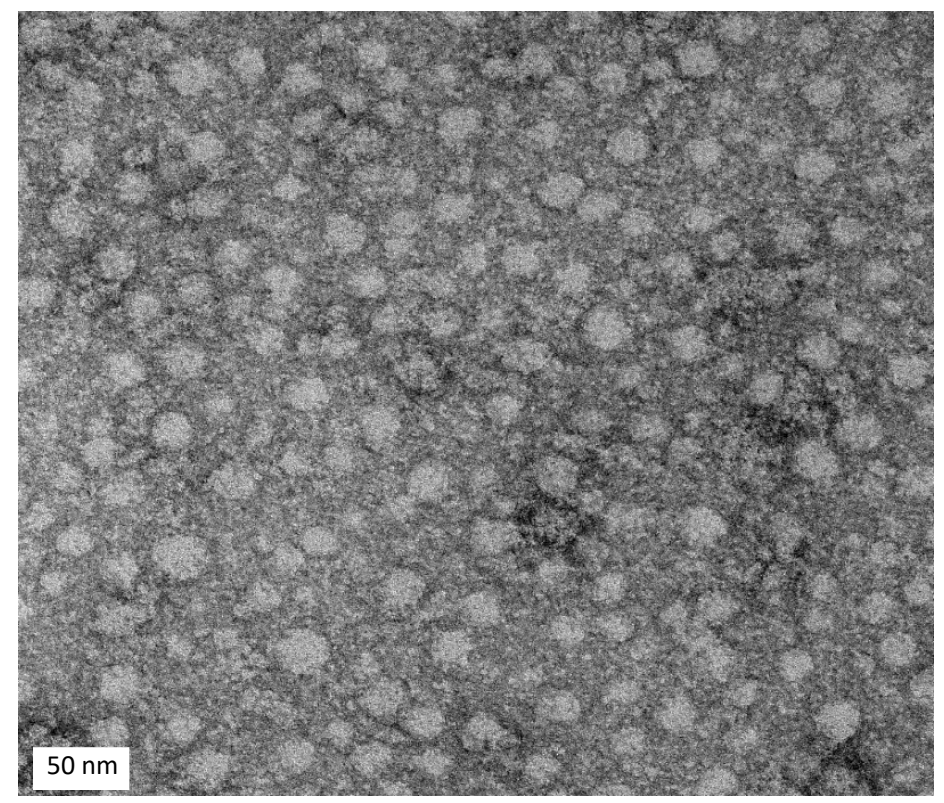

Figure 4. TEM micrographs of GMA-EGDMA-S ${ }^{+}$give a mean particle diameter of $29 \pm 10 \mathrm{~nm}$. The scale bar represents $50 \mathrm{~nm}$.

\subsubsection{Controlled epoxide conversion}

Particles with high charge density are known to exhibit cytotoxicity. ${ }^{75}$ Therefore, control over the degree of functionalization with charged moieties is desired. By investigating the kinetics of GMA-EGDMA-S ${ }^{+}$synthesis, a certain degree of functionalization and thus positive charge can be obtained by quenching the reaction at a certain time point. The evolution of characteristic ${ }^{1} \mathrm{H}$ NMR signals for GMA-EGDMA-S ${ }^{+}$during the diethyl sulfide induced ring-opening reaction is shown in Figure 5. 


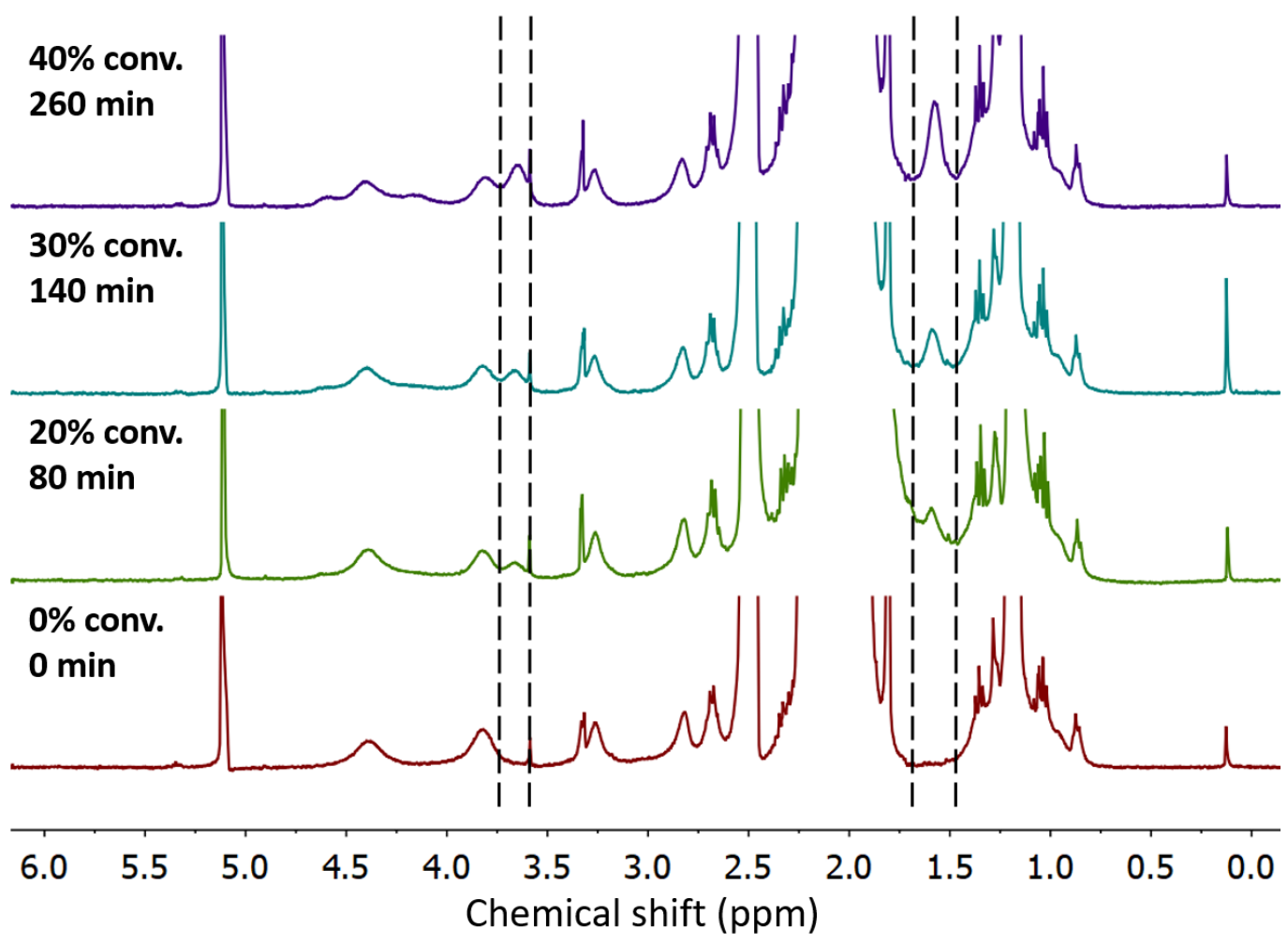

Figure 5. Conversion of epoxides during a ring-opening reaction of GMA-EGDMA-S+ with 5 equiv. of diethyl sulfide in $\mathrm{AcOH} /$ acetone $1: 1$, as analyzed by ${ }^{1} \mathrm{H}$ NMR spectroscopy in acetone- $\mathrm{d}_{6}$. The characteristic signals of the sulfonium group at $\delta=1.4 \mathrm{ppm}$ and $\delta=3.5 \mathrm{ppm}$ increase with time. Conversion was calculated utilizing 1,3,5-trioxane as an internal standard. Its singlet peak can be seen at $5.1 \mathrm{ppm}$.

\subsubsection{Solubility of sulfonium-functionalized nanogels}

Having demonstrated the control over conversion and thus charge density, a series of GMA-EGDMA-S ${ }^{+}$nanogels were made at various epoxide conversions. Surprisingly, relatively high conversions were needed to achieve water-solubility. At $50 \%$ epoxide 
conversion solubility was achieved at a concentration of $0.5 \mathrm{mg} / \mathrm{mL}$ in water after $30 \mathrm{~min}$ of sonication.
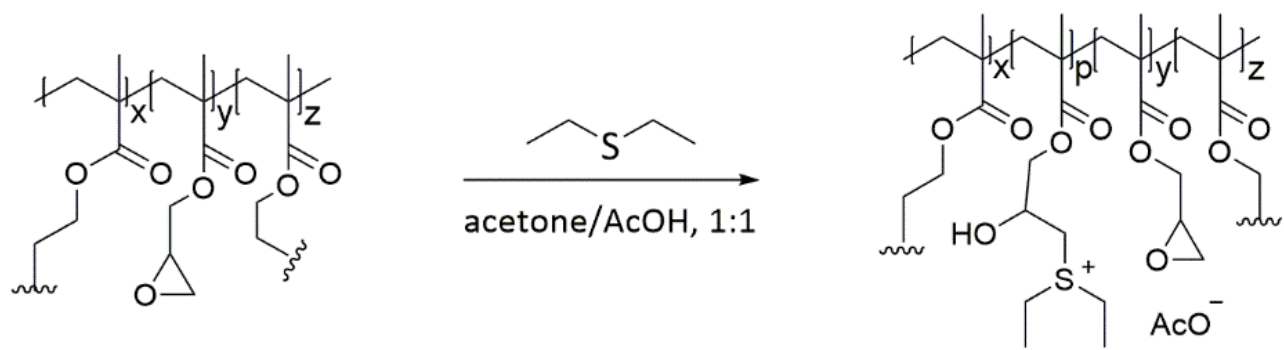

Scheme 4. The synthesis of GMA-EGDMA-S+-x, $x$ denoting the epoxide conversion.

Table 1. GMA-EGDMA-S+-x nanogels were synthesized and their solubility in water was probed. The nanogels became appreciably water-soluble at $50 \%$ epoxide conversion.

\begin{tabular}{|l|l|}
\hline Conversion (\%) & Water-soluble \\
\hline 15 & no \\
\hline 25 & no \\
\hline 30 & no \\
\hline 45 & no \\
\hline 50 & yes \\
\hline
\end{tabular}


As these nanogels are to be used in the human body for transfection purposes, watersolubility is a strict requirement. The remaining epoxides of GMA-EGDMA-S $\mathbf{S}^{+}-\mathbf{x}$ provide a convenient handle for further nanogel functionalizations to enhance water-solubility. Coincidentally this strategy also increases the biocompatibility of these nanogels as epoxides have been proven to be toxic. ${ }^{76}$

Having demonstrated in Chapter 3 the versatility and ease of epoxide ring opening reactions, it was decided to apply these strategies on GMA-EGDMA-S+-x.

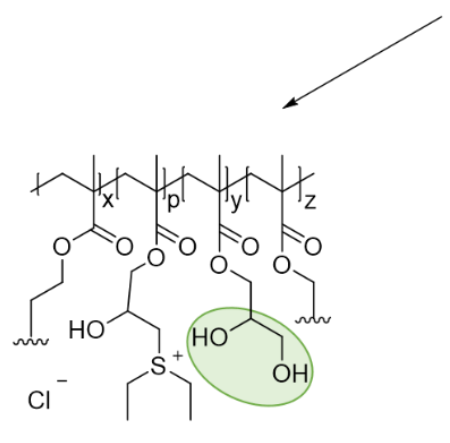

GMA-EGDMA-S+-OH
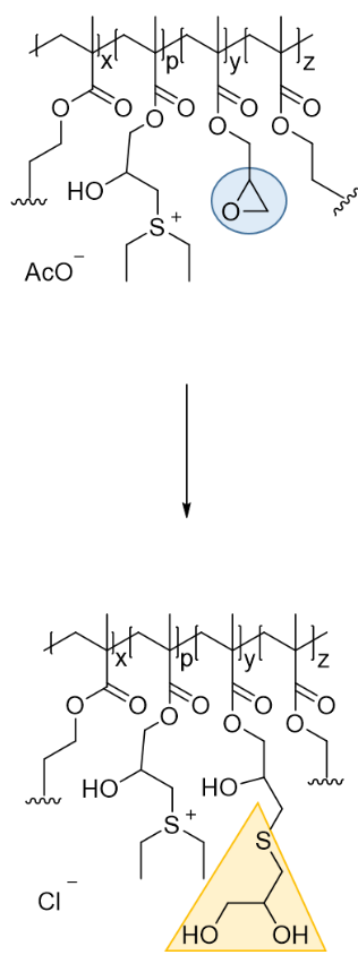

GMA-EGDMA-S+-THG

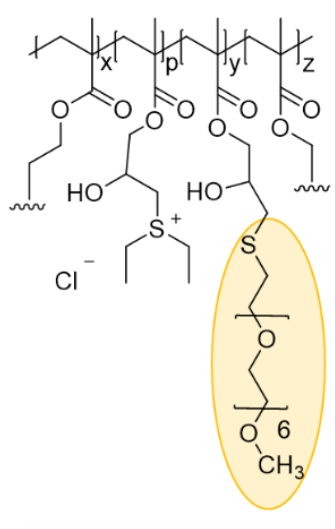

GMA-EGDMA-S+-mPEG

Scheme 5. Water-solubility of GMA-EGDMA-S+-x was attempted by hydrolyzing the remaining epoxides with TFA to GMA-EGDMA-S+-OH and by subjecting them to ring opening reactions with hydrophilic thiols to GMAEGDMA-S+-THG and GMA-EGDMA-S+-mPEG. 


\subsubsection{Hydrolysis of epoxides using TFA}

As a first strategy, hydrolysis of the remaining epoxides was carried out in an attempt to solubilize GMA-EGDMA-S'-x nanogels at low charge densities. The nanogel was reacted with TFA in 9:1 THF/water. During this step the acetate counterion is replaced by a trifluoroacetate counterion as excess TFA (12 equivalents to epoxide groups) is used. However, this counterion has been reported as toxic in cell culture experiments by inhibition of proliferation. ${ }^{77}$ Therefore, dialysis against $0.1 \mathrm{M} \mathrm{NaCl}$ was done to facilitate counterion exchange to $\mathrm{Cl}^{-}$. The disappearance of the $\mathrm{AcO}^{-1} \mathrm{H}$ NMR signal at $\delta=1.9 \mathrm{ppm}$ indicated a successful exchange. Nanogels GMA-EGDMA-S+-10, GMA-EGDMA-S+-15 and GMA-EGDMA-S'-25 (with 10, 15, 25 denoting the epoxide conversion) all formed insoluble crosslinked products. GMA-EGDMA-S ${ }^{+}-45$ was successfully hydrolyzed to GMAEGDMA-S+-45-OH. IR measurements, showed no characteristic epoxide band at $907 \mathrm{~cm}^{-1}$, indicating complete hydrolysis of epoxides. Although the exact mechanism behind crosslinking during acidic hydrolysis with a low charge density is not known, it is suspected that particles with higher charge density experience more electrostatic repulsion, preventing crosslinking due to decreased proximity. GMA-EGDMA-S ${ }^{+}-45-\mathrm{OH}$ was watersoluble. DLS and zeta potential measurements gave a hydrodynamic diameter of $121 \mathrm{~nm}$ and a surface charge of $+34 \mathrm{mV}$ at neutral $\mathrm{pH}$. As hydrolysis of the epoxides is troublesome for nanogels with low degrees of charge density, other reactions were investigated.

\subsubsection{Thioglycerol-epoxy ring-opening}

Thiol-epoxy ring-opening reactions are known as click chemistry reactions, proceeding with high efficiency at ambient temperatures. ${ }^{78}$ Therefore, thiol-epoxy reactions were investigated for the solubilization of GMA-EGDMA-S+-x. To enhance hydrophilicity, 1thioglycerol was used during a ring-opening reaction under basic conditions. 
Unfortunately these nanogels were also not water-soluble at a concentration of 0.5 $\mathrm{mg} / \mathrm{mL}$. Cell transfection experiments can be carried out at lower nanogel concentrations - however, $0.5 \mathrm{mg} / \mathrm{mL}$ is the minimum required for accurate characterization.

\subsection{7 mPEG thiol-epoxy ring opening}

PEGylation is a well-known strategy to improve water-solubility of particles. ${ }^{79}$ PEGylated particles are also known to have an increased blood circulation half-life. Therefore, a thiolfunctionalized PEG oligomer (O-(2-mercaptoethyl)-O'-methyl-hexa(ethylene glycol)) was employed in efforts to solubilize GMA-EGDMA-S ${ }^{+}-\mathbf{x}$. To prevent crosslinking, a thiolfunctionalized PEG oligomer with six repeating ethylene glycol units and methoxy endgroups (mPEG) was chosen. Longer mPEG units are expected to experience a greater degree of steric hindrance when attacking epoxides within the nanogel matrix. Conversely, shorter MPEG units might not yield water-soluble nanogels due to their relatively low contribution to overall hydrophilicity of the nanogels. The degree of conversion could not be determined by ${ }^{1} \mathrm{H}$ NMR spectroscopy due to overlapping signals in the $2.5-3.5 \mathrm{ppm}$ region. These NMR spectra however are in agreement with the one obtained in the work of De et al. ${ }^{78}$, who used a similar approach to attach thiolated mPEG chains to polymers with epoxide moieties. Sulfonium-mPEG functionalized nanogels GMA-EGDMA-S ${ }^{+}$-27-mPEG and GMA-EGDMA-S+-47-mPEG were synthesized. These compounds, however, exhibited poor water-solubility as well at $0.5 \mathrm{mg} / \mathrm{mL}$. DLS indicated the presence of large aggregates. 


\subsubsection{Probing nanogel solubility by tuning its crosslinker}

The experiments described above irrefutably indicate that proper solvation of GMAEGDMA-S+-x nanogels at low positive charge density is not achievable solely by functionalization of the remaining epoxides with hydrophilic reactants. The remainder of the nanogel matrix is probably too hydrophobic. Another strategy would be reducing this hydrophobicity, for example by using a more hydrophilic crosslinker during nanogel synthesis.

Test reactions were carried out with $N, N^{\prime}$-methylenebisacrylamide (MBA), diethylene glycol dimethacrylate (DEGDMA) and tetraethylene glycol dimethacrylate (TTEGDMA) as crosslinker at varying conditions. MBA did not form well-defined nanogels as it is not compatible with GMA - most likely due to differing reactivity ratios. DEGDMA and TTEGDMA were successfully crosslinked with GMA to form nanogels. The remaining methacrylate groups were not quenched as they do not hinder the reaction with diethyl sulfide or cause crosslinking.

1

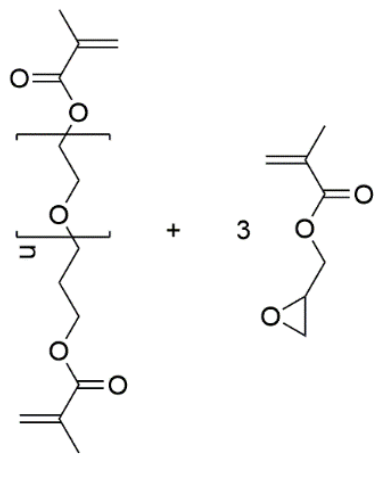

$\mathrm{n}=1$ or 3

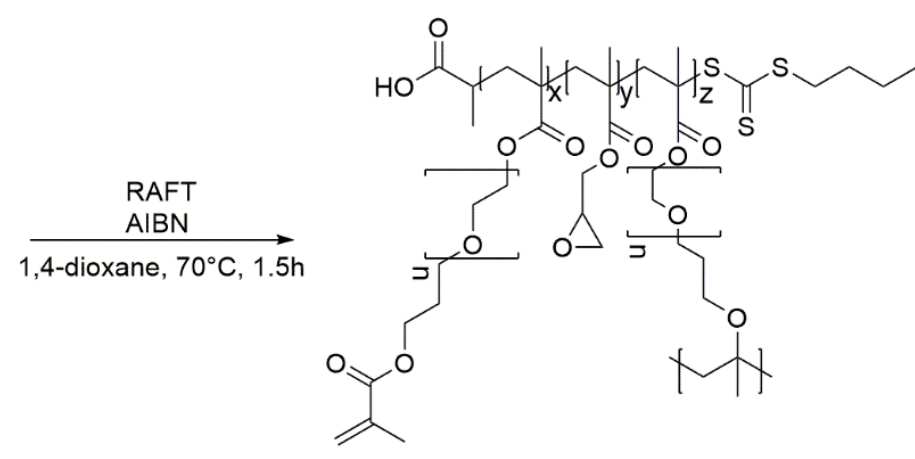

$\mathrm{n}=1$ or 3

Scheme 6. The RAFT polymerization of DEGDMA and TTEGDMA with GMA forming GMA-DEGDMA $(n=1)$ and GMA-TTEGDMA $(n=3)$ nanogels. 
In a next step, these two nanogels were subjected to epoxide ring opening reaction with diethyl sulfide to form GMA-DEGDMA-S ${ }^{+}-\mathbf{x}$ and GMA-TTEGDMA-S+-x at low epoxide conversions (low value for $\mathrm{x}$ ) and evaluate their water-solubility.

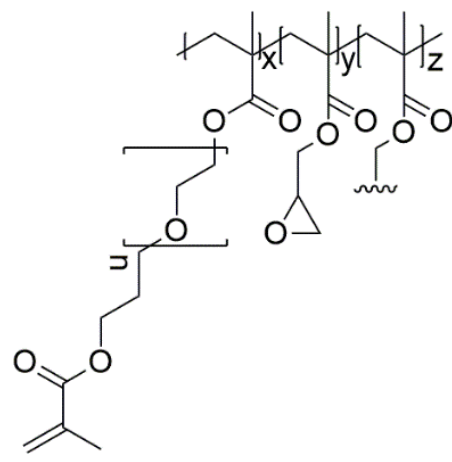

$\mathrm{n}=1$ or 3

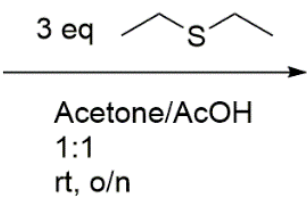

$\mathrm{O}$<smiles>C=C(C)C(=O)OC</smiles>

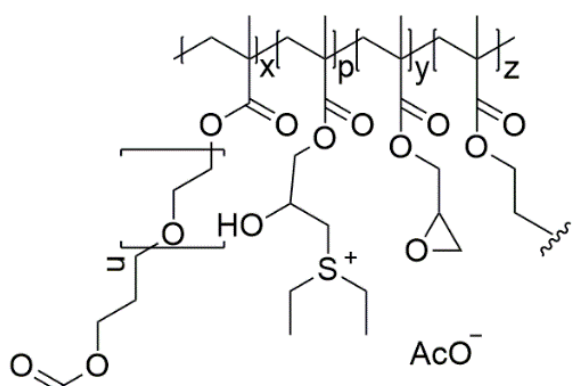

$\mathrm{n}=1$ or 3

Scheme 7. GMA-DEGDMA $(n=1)$ and GMA-TTEGDMA $(n=3)$ were reacted with 3 equivalents of diethyl sulfide to form GMA-DEGDMA-S ${ }^{+-x}$ and GMA-TTEGDMA-S+-x.

Both reactions were successful, however, only GMA-TTEGDMA-S+-15 was water-soluble at $1.3 \mathrm{mg} / \mathrm{mL}$ in. ${ }^{1} \mathrm{H}$ NMR spectroscopy indicated an epoxide conversion of $15 \%$. 


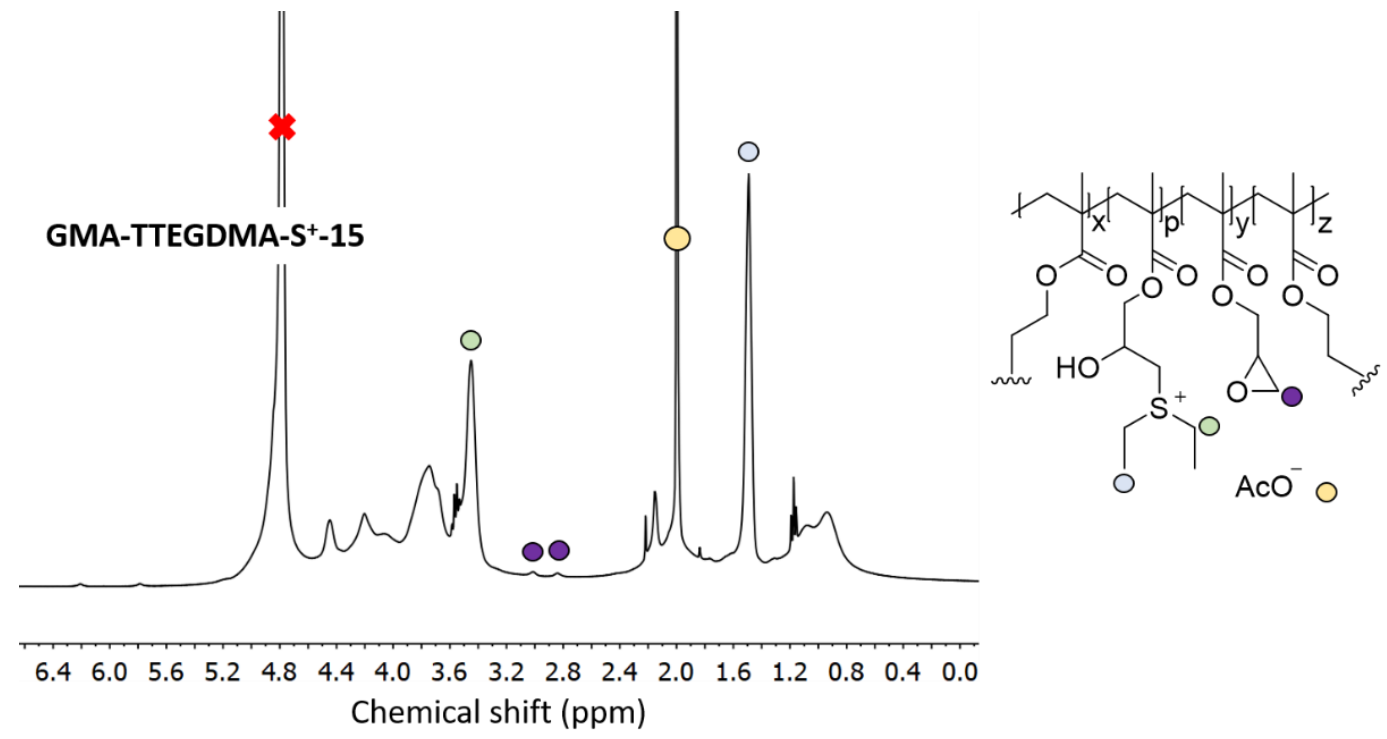

Figure 6. ${ }^{1} \mathrm{H}$ NMR spectrum of GMA-TTEGDMA-S+-15 in $\mathrm{D}_{2} \mathrm{O}$ displays the characteristic sulfonium peaks at $\delta$ $=1.4 \mathrm{ppm}$ and $\delta=3.5 \mathrm{ppm}$ along with the remaining epoxide peaks at $\delta=2.6 \mathrm{ppm}$ and $\delta=2.8 \mathrm{ppm}$ (the epoxide peak at $\delta=3.2 \mathrm{ppm}$ is hidden under the methylene peaks of the sulfonium group). The acetate counter ion can also be seen at $\delta=1.9 \mathrm{ppm}$. The structure of the nanogel was simplified to enhance clarity.

Utilizing TTEGDMA as crosslinker during nanogel formation with GMA increases the hydrophilicity of the nanogel and its water-solubility at low surface charge density.

\subsection{Conclusions}

The objective of this work was to synthesize sulfonium-functionalized nanogels for gene delivery applications. GMA-EGDMA nanogels were synthesized by a RAFT CCP approach followed by methacrylate quenching.

Sulfonium groups were installed by applying a ring-opening reaction of epoxide moieties with diethyl sulfide under acidic conditions. The degree of functionalization was found to be controllable by monitoring the conversion with NMR spectroscopy. 
The solubility of sulfonium functionalized nanogels GMA-EGDMA-S'-x in water was found to be heavily dependent on the degree of functionalization $(\mathbf{x})$ and thus the amount of positive charge. Several approaches were investigated to solubilize sulfoniumfunctionalized nanogels GMA-EGDMA-S $\mathbf{S}^{+} \mathbf{x}$ by utilizing the remaining epoxides as a reactive moiety. Hydrolysis of epoxides with TFA was found to induce crosslinking by a so far unknown mechanism. Thiol-epoxy ring-opening reactions with 1-thioglycerol and a thiol-functionalized mPEG oligomers were either not successful or did not give appreciably water-soluble products.

Substituting the crosslinker EGDMA for its longer and more hydrophilic equivalent TTEGDMA resulted in GMA-TTEGDMA nanogels that once functionalized with a sulfonium moiety are water-soluble starting from 15\% epoxide conversion (GMA-TTEGDMA-S+15).

The work presented here serves as a foundation for the synthesis of sulfoniumfunctionalized nanogels with tunable size and charge density, potentially serving as nontoxic efficient gene carriers.

\subsection{Acknowledgements}

First and foremost Thijs van Veldhuisen is recognized for his hard work and dedication to this project. Dr. E. G. Keim is acknowledged for his help with TEM measurements along with Dr. Michel Klein Gunnewiek for his help with the AFM measurements. Dr. Anzar Khan and Taejun Eom are thanked for their guidance in the epoxide chemistry. 


\subsection{Experimental section}

Materials. Glycidyl methacrylate (GMA, 97\%), ethylene glycol dimethacrylate (EGDMA, 98\%), diethylene glycol dimethacrylate (DEGDMA, 95\%) and tetraethylene glycol dimethacrylate (TTEGDMA, >90\%) were purchased from Sigma-Aldrich and passed through a column of neutral $\mathrm{Al}_{2} \mathrm{O}_{3}$ prior to use. Potassium chloride $(\mathrm{KCl}), \mathrm{N}, \mathrm{N}-$ dimethylformamide (DMF, anhydrous, 99.8\%), trifluoroacetic acid (TFA, HPLC grade, $>99.0 \%$ ), 1,4-dioxane (HPLC grade, >99.5\%), azobisisobutyronitrile (AIBN, 98\%) and lithium hydroxide ( $\mathrm{LiOH}$, reagent grade, 98\%) were purchased from Sigma-Aldrich and used without further purification. Dichloromethane (DCM, 99.7\%) and methanol (MeOH) were obtained from Ossum Chemicals. The RAFT agent 2-[[(butylthio)thioxomethyl] thio] propanoic acid was synthesized following a literature procedure. ${ }^{80}$ Deuterated solvents were purchased from Sigma-Aldrich and used as received. The water used in this work was treated through a Milli-Q Gradient System. Monomers containing hydroquinone as inhibitor were filtered over aluminium oxide 90 neutral before use. Dialysis was carried out using SnakeSkin dialysis tubing with a molecular weight cut-off of $10 \mathrm{kDa}$.

Analysis. Lyophilization was performed using a Labconco FreeZone 4.5 Liter Benchtop Freeze Dry System. NMR spectra were recorded on a Bruker Ascend $400 \mathrm{MHz}$ NMR spectrometer at $298 \mathrm{~K}$. Residual solvent signals were used as internal reference according to the literature. ${ }^{81}$ Multiplicities are abbreviated as follows: singlet (s), doublet (d), and multiplet (m). IR spectra were recorded on a Nicolet 6700 FT-IR spectrometer from Thermo Scientific, equipped with a Smart Orbit Diamond Attenuated Total Reflectance accessory. DLS and zeta potential measurements were carried out on a Malvern Zetasizer 4000, performed in triplicate. As a reference material for DLS, poly(ethylene glycol)-co-poly(isobutyl methacrylate) with a refractive index (RI) of 1.465 was used. DLS measurements were carried with concentrations in the range of 0.5-1.0 $\mathrm{mg} / \mathrm{mL}$. Zeta potential measurements were carried out in Milli-Q in the presence of $5 \mathrm{mM}$ 
$\mathrm{KCl}$ or in $10 \mathrm{mM}$ HEPES buffer at $\mathrm{pH}=7$. TEM was carried out on a Philips CM300ST-FEG Transmission Electron Microscope. Samples were prepared on Electron Microscopy Sciences FCF200-Cu Formvar/copper support grids (200 mesh). Solutions of nanogels were prepared with concentrations of $0.5 \mathrm{mg} / \mathrm{mL}$ in water or 1,4-dioxane and sonicated for $30 \mathrm{~min} .5 \mu \mathrm{L}$ of the solution was deposited on the grid and dried immediately using a paper filter. After drying of the grid in the fume hood, $5 \mu \mathrm{L}$ of an aqueous $1 \% \mathrm{w} / \mathrm{v}$ uranyl acetate solution was deposited on the grid and dried after 30 seconds, using a paper filter. Then, samples were dried in the fume hood. Particle size analysis using TEM micrographs was carried out using ImageJ software by calibrating to the scale given in the micrographs and measuring at least 200 particles.

\subsubsection{Nanogel synthesis}

GMA-EGDMA nanogel formation. In a typical procedure, GMA $(2.1 \mathrm{~mL}, 16 \mathrm{mmol}$, 3.0 equiv.), EGDMA (1.0 mL, $5.3 \mathrm{mmol}, 1.0$ equiv), AIBN (1.4 mL, $61 \mathrm{mM}$ in 1,4-dioxane, $8.4 \mu \mathrm{mol}, 1.6 \mathrm{~mol} \%)$, and 2-(((butylthio)carbonothioyl)thio)propanoic acid (1.0 mL, 0.42 $M$ in 1,4-dioxane, $0.42 \mathrm{mmol}, 8.0 \mathrm{~mol} \%)$ were added to a flask with $50 \mathrm{~mL}$ of 1,4-dioxane, giving a monomer concentration of $6 \% \mathrm{w} / \mathrm{w}$. The flask was sealed with a septum and degassed by nitrogen bubbling for $30 \mathrm{~min}$. Then, the reaction mixture was heated to 70 ${ }^{\circ} \mathrm{C}$ and stirred at $200 \mathrm{rpm}$ for $90 \mathrm{~min}$. An excess of AIBN (4.6 gram, $28 \mathrm{mmol}, 5.3$ equiv.) was dissolved in $30 \mathrm{~mL}$ of 1,4-dioxane. This AIBN solution was degassed by nitrogen bubbling for $30 \mathrm{~min}$ and added to the reaction mixture. After $22 \mathrm{~h}$ of stirring at $70^{\circ} \mathrm{C}$, the reaction mixture was precipitated three times in hexane. The solids were dried under a stream of nitrogen yielding white solids (1.33 gram, quantitative yield). Conversion of monomers was determined by ${ }^{1} \mathrm{H}$ NMR spectroscopy using 1,4-dioxane as an internal 
standard for relative signal intensity. ${ }^{1} \mathrm{H}$ NMR spectroscopy at 90 min gave a monomer conversion of $30 \%$.

${ }^{1} \mathrm{H}$ NMR (GMA-EGDMA, $\left.400 \mathrm{MHz}, \mathrm{CDCl}_{3}\right): \delta(\mathrm{ppm}): 4.67-4.06\left(\mathrm{~m}, \mathrm{CH}_{2}\right)$, 4.05-3.55 (m, $\mathrm{CH}_{2}$ ), 3.54-3.07 ( $\mathrm{m}, \mathrm{CH}$, epoxide), 3.05-2.76 ( $\mathrm{m}, \mathrm{CH}_{2}$, epoxide), 2.76-2.50 ( $\mathrm{m}, \mathrm{CH}_{2}$, epoxide), 2.29$1.81\left(\mathrm{~s}, \mathrm{CH}_{2}\right), 2.21-1.67\left(\mathrm{~s}, \mathrm{CH}_{2}\right), 1.47-0.65\left(\mathrm{~m}, \mathrm{CH}_{3}\right)$.

IR (GMA-EGDMA, neat) $\lambda_{\text {max: }}$ 2990, 2952, 1724, 1450, 1387, 1257, 1146, 993, 906, 848, $759 \mathrm{~cm}^{-1}$.

GMA-DEGDMA nanogel formation. RAFT agent 2-[[(butylthio)thioxomethyl] thio] propanoic acid (22.1 mg, $0.1 \mathrm{mmol}, 1$ equiv.), AIBN ( $3 \mathrm{mg}, 0.02 \mathrm{mmol}, 0.2$ equiv.), GMA (0.46 mL, $3.5 \mathrm{mmol}, 37.5$ equiv.), DEGDMA (0.26 mL, $1.2 \mathrm{mmol}, 12.5$ equiv.) and 1,4dioxane (10 mL, $93 \mathrm{w} / \mathrm{w} \%$ ) were added to a $25 \mathrm{~mL}$ round bottom flask equipped with a stir bar, sealed with a septum and purged with nitrogen for 30 min. The flask was subsequently placed in an oil bath at $70{ }^{\circ} \mathrm{C}$ and allowed to react for $1.5 \mathrm{~h}$ after which it was taken out of the oil bath and quenched with $3 \mathrm{~mL} \mathrm{DCM}$. The nanogel was precipitated three times in hexane yielding a white solid $(235.9 \mathrm{mg}, 35 \%$ conversion, $8.73 \mathrm{mmol} / \mathrm{g}$ epoxides).

GMA-TTEGDMA nanogel formation. RAFT agent 2-[[(butylthio)thioxomethyl] thio] propanoic acid ( $19.5 \mathrm{mg}, 0.08 \mathrm{mmol}, 1$ equiv.), AIBN ( $2.7 \mathrm{mg}, 0.02 \mathrm{mmol}, 0.2$ equiv.), GMA (0.41 mL, $3.1 \mathrm{mmol}, 37.5$ equiv.), TTEGDMA (0.31 mL, $1 \mathrm{mmol}, 12.5$ equiv.), DMF (0.24 mL, $3 \mathrm{mmol}, 37.5$ equiv.) as internal standard and 1,4-dioxane (10 mL, $93 \mathrm{w} / \mathrm{w} \%)$ were added to a $25 \mathrm{~mL}$ round bottom flask equipped with a stir bar, sealed with a septum and purged with nitrogen for 30 min. The flask was subsequently placed in an oil bath at $70{ }^{\circ} \mathrm{C}$ and allowed to react for $1.5 \mathrm{~h}$ after which it was taken out of the oil bath and quenched with $6 \mathrm{~mL}$ DCM. The nanogel was precipitated twice in hexane yielding a white solid (187.3 mg, 27\% conversion, $4.38 \mathrm{mmol} / \mathrm{g}$ epoxides). 


\subsubsection{Nanogel functionalization}

Sulfonium nanogels. In a typical procedure, GMA-EGDMA $(68 \mathrm{mg}, 0.44 \mathrm{mmol}$ of epoxides) was dissolved in a mixture of $\mathrm{AcOH} /$ acetone $(1: 1,3.2 \mathrm{~mL})$. To the flask, diethyl sulfide ( $250 \mu \mathrm{L}, 2.3 \mathrm{mmol}, 7.0$ equiv.) was added. After stirring for $230 \mathrm{~min}$, the product was purified by precipitation into ice-cold diethyl ether twice. After drying under a stream of nitrogen, GMA-EGDMA-S ${ }^{+}-\mathbf{x}$ was obtained as a glassy colorless solid.

${ }^{1} \mathrm{H}_{\text {NMR (GMA-EGDMA-S }}^{+}, 400 \mathrm{MHz}$, DMSO-d $\left.\mathrm{d}_{6}\right): \delta(\mathrm{ppm}):$ 6.95-6.13 (s, OH), 4.68-2.79 (m, $\mathrm{CH}$ and $\left.\mathrm{CH}_{2}\right), 2.28-1.83\left(\mathrm{~s}, \mathrm{CH}_{2}\right), 1.79-0.44\left(\mathrm{~m}, \mathrm{CH}_{3}\right)$.

Hydrolyzed sulfonium nanogels. In a typical procedure, GMA-EGDMA-S+-45 (119 $\mathrm{mg}, 0.31 \mathrm{mmol}$ of epoxides) was dissolved in a mixture of tetrahydrofuran (THF) and water (9:1, $10 \mathrm{~mL})$. To the mixture, TFA ( $280 \mu \mathrm{L}, 3.6 \mathrm{mmol}, 12$ equiv. to epoxides) was added. The reaction mixture was stirred at room temperature for 7 days. Next, the reaction mixture was dialyzed against $0.1 \mathrm{M} \mathrm{NaCl}$ for 2 days and against Milli-Q for 4 days. After lyophilization, GMA-EGDMA-S+-45-OH was obtained as white solids ( $84 \mathrm{mg}, 67 \%$ yield).

${ }^{1}{ }_{H}$ NMR (GMA-EGDMA-S $-45-O H, 400 \mathrm{MHz}$, DMSO-d $\mathrm{d}_{6}$ ): $\delta$ (ppm): 6.72-6.03 (s, OH), 5.75$4.50(\mathrm{~m}, \mathrm{OH}), 4.50-3.37\left(\mathrm{~m}, \mathrm{CH}_{2}\right.$ and $\left.\mathrm{CH}\right), 2.26-1.80\left(\mathrm{CH}_{2}\right), 1.65-1.24\left(\mathrm{~s}, \mathrm{CH}_{3}\right), 1.24-0.41(\mathrm{~m}$, $\left.\mathrm{CH}_{3}\right)$.

IR (GMA-EGDMA-S ${ }^{+}-45-O H$, neat): $\lambda_{\max }: 3368$ (broad), 2944, 1723, 1456, 1387, 1263, $1154,1051,978,938,752 \mathrm{~cm}^{-1}$.

Thioglycerol sulfonium nanogels. In a typical procedure, GMA-EGDMA-S ${ }^{+}-39$ (98 $\mathrm{mg}, 0.47 \mathrm{mmol}$ of epoxides, 1.0 equiv.) and $\mathrm{LiOH}(14 \mathrm{mg}, 0.58 \mathrm{mmol}, 1.2$ equiv.) were dissolved in a mixture of dimethylformamide (DMF) and water $(9: 1,9 \mathrm{~mL})$. To the mixture, 1-thioglycerol (100 $\mu \mathrm{L}, 1.15 \mathrm{mmol}, 2.4$ equiv.) was added. The reaction mixture was stirred at room temperature for 4 days. Next, the reaction mixture was dialyzed against $0.1 \mathrm{M}$ 
$\mathrm{NaCl}$ for $24 \mathrm{~h}$ and against Milli-Q for $48 \mathrm{~h}$. After lyophilization, GMA-EGDMA-S+-39-THG was obtained as white solids ( $121 \mathrm{mg}, 88 \%$ yield).

Pegylated sulfonium nanogels. In a typical procedure, GMA-EGDMA-S ${ }^{+}-47$ (30 $\mathrm{mg}, 76 \mu \mathrm{mol}$ of epoxides, 1.0 equiv.) and $\mathrm{LiOH}(5.6 \mathrm{mg}, 0.23 \mathrm{mmol}, 3.1$ equiv.) were dissolved in a mixture of DMF and water $(9: 1,10 \mathrm{~mL})$. To the mixture, $O-(2-$ mercaptoethyl)-O'-methyl-hexa(ethylene glycol) (33 $\mu \mathrm{L}, 99 \mu \mathrm{mol}, 1.3$ equiv.) was added. The reaction mixture was stirred at room temperature for 2 days. Next, the reaction mixture was dialyzed against $0.1 \mathrm{M} \mathrm{NaCl}$ for 2 days and against Milli-Q for 5 days. After lyophilization, GMA-EGDMA-S+-47-mPEG was obtained as white solids ( $34 \mathrm{mg}$, 52\% yield).

\subsection{References}

1. Rossi, J. J.; June, C. H.; Kohn, D. B., Genetic therapies against HIV. Nature Biotechnology 2007, 25 (12), 1444-1454.

2. Qin, X.-F.; An, D. S.; Chen, I. S. Y.; Baltimore, D., Inhibiting HIV-1 infection in human T cells by lentiviral-mediated delivery of small interfering RNA against CCR5. Proceedings of the National Academy of Sciences 2003, 100 (1), 183.

3. Amer, M. H., Gene therapy for cancer: present status and future perspective. Mol Cell Ther 2014, 2, 27.

4. Cross, D.; Burmester, J. K., Gene Therapy for Cancer Treatment: Past, Present and Future. Clinical Medicine \& Research 2006, 4 (3), 218-227.

5. Goswami, R.; Subramanian, G.; Silayeva, L.; Newkirk, I.; Doctor, D.; Chawla, K.; Chattopadhyay, S.; Chandra, D.; Chilukuri, N.; Betapudi, V., Gene Therapy Leaves a Vicious Cycle. Frontiers in Oncology 2019, 9 (297).

6. Ibraheem, D.; Elaissari, A.; Fessi, H., Gene therapy and DNA delivery systems. International Journal of Pharmaceutics 2014, 459 (1), 70-83. 
7. Lam, J. K. W.; Chow, M. Y. T.; Zhang, Y.; Leung, S. W. S., siRNA Versus miRNA as Therapeutics for Gene Silencing. Molecular Therapy - Nucleic Acids 2015, 4.

8. Ahmadzada, T.; Reid, G.; McKenzie, D. R., Fundamentals of siRNA and miRNA therapeutics and a review of targeted nanoparticle delivery systems in breast cancer. Biophysical Reviews 2018, 10 (1), 69-86.

9. Lechardeur, D.; Sohn, K. J.; Haardt, M.; Joshi, P. B.; Monck, M.; Graham, R. W.; Beatty, B.; Squire, J.; O’Brodovich, H.; Lukacs, G. L., Metabolic instability of plasmid DNA in the cytosol: a potential barrier to gene transfer. Gene Therapy 1999, 6 (4), 482-497.

10. Singh, A.; Trivedi, P.; Jain, N. K., Advances in siRNA delivery in cancer therapy. Artificial cells, nanomedicine, and biotechnology 2018, 46 (2), 274-283.

11. Herweijer, H.; Wolff, J. A., Progress and prospects: naked DNA gene transfer and therapy. Gene Therapy 2003, 10 (6), 453-458.

12. Somia, N.; Verma, I. M., Gene therapy: trials and tribulations. Nature Reviews Genetics 2000, 1 (2), 91-99.

13. Wong, S. Y.; Pelet, J. M.; Putnam, D., Polymer systems for gene delivery-Past, present, and future. Progress in Polymer Science 2007, 32 (8), 799-837.

14. Nel, A. E.; Mädler, L.; Velegol, D.; Xia, T.; Hoek, E. M. V.; Somasundaran, P.; Klaessig, F.; Castranova, V.; Thompson, M., Understanding biophysicochemical interactions at the nano-bio interface. Nature Materials 2009, 8 (7), 543-557.

15. Venturoli, D.; Rippe, B., Ficoll and dextran vs. globular proteins as probes for testing glomerular permselectivity: effects of molecular size, shape, charge, and deformability. American Journal of Physiology-Renal Physiology 2005, 288 (4), F605-F613.

16. Kulkarni, S. A.; Feng, S.-S., Effects of Particle Size and Surface Modification on Cellular Uptake and Biodistribution of Polymeric Nanoparticles for Drug Delivery. Pharmaceutical Research 2013, 30 (10), 2512-2522. 
17. Hoshyar, N.; Gray, S.; Han, H.; Bao, G., The effect of nanoparticle size on in vivo pharmacokinetics and cellular interaction. Nanomedicine (Lond) 2016, 11 (6), 673692.

18. Nakai, T.; Kanamori, T.; Sando, S.; Aoyama, Y., Remarkably size-regulated cell invasion by artificial viruses. Saccharide-dependent self-aggregation of glycoviruses and its consequences in glycoviral gene delivery. Journal of the American Chemical Society 2003, 125 (28), 8465-8475.

19. Kabanov, A. V., Taking polycation gene delivery systems from in vitro to in vivo. Pharmaceutical Science \& Technology Today 1999, 2 (9), 365-372.

20. Vinogradov, S. V.; Bronich, T. K.; Kabanov, A. V., Nanosized cationic hydrogels for drug delivery: preparation, properties and interactions with cells. Advanced Drug Delivery Reviews 2002, 54 (1), 135-147.

21. Ogawara, K.-I.; Yoshida, M.; Furumoto, K.; Takakura, Y.; Hashida, M.; Higaki, K.; Kimura, T., Uptake by hepatocytes and biliary excretion of intravenously administered polystyrene microspheres in rats. Journal of drug targeting 1999, 7 (3), 213-221.

22. Delenda, C., Lentiviral vectors: optimization of packaging, transduction and gene expression. The Journal of Gene Medicine 2004, 6 (S1), S125-S138.

23. Milone, M. C.; O'Doherty, U., Clinical use of lentiviral vectors. Leukemia 2018, 32 (7), 1529-1541.

24. Lee, C. S.; Bishop, E. S.; Zhang, R.; Yu, X.; Farina, E. M.; Yan, S.; Zhao, C.; Zeng, Z.; Shu, Y.; Wu, X.; Lei, J.; Li, Y.; Zhang, W.; Yang, C.; Wu, K.; Wu, Y.; Ho, S.; Athiviraham, A.; Lee, M. J.; Wolf, J. M.; Reid, R. R.; He, T.-C., Adenovirus-mediated gene delivery: Potential applications for gene and cell-based therapies in the new era of personalized medicine. Genes \& Diseases 2017, 4 (2), 43-63.

25. Huang, X.; Yang, Y., Innate immune recognition of viruses and viral vectors. Human gene therapy 2009, 20 (4), 293-301. 
26. Shirley, J. L.; de Jong, Y. P.; Terhorst, C.; Herzog, R. W., Immune Responses to Viral Gene Therapy Vectors. Molecular Therapy 2020, 28 (3), 709-722.

27. Felgner, P. L.; Gadek, T. R.; Holm, M.; Roman, R.; Chan, H. W.; Wenz, M.; Northrop, J. P.; Ringold, G. M.; Danielsen, M., Lipofection: a highly efficient, lipid-mediated DNA-transfection procedure. Proceedings of the National Academy of Sciences 1987, 84 (21), 7413.

28. Wasungu, L.; Hoekstra, D., Cationic lipids, lipoplexes and intracellular delivery of genes. Journal of Controlled Release 2006, 116 (2), 255-264.

29. Koltover, I.; Salditt, T.; Rädler, J. O.; Safinya, C. R., An Inverted Hexagonal Phase of Cationic Liposome-DNA Complexes Related to DNA Release and Delivery. Science 1998, 281 (5373), 78.

30. Liu, F.; Qi, H.; Huang, L.; Liu, D., Factors controlling the efficiency of cationic lipidmediated transfection in vivo via intravenous administration. Gene Therapy 1997, $4(6), 517-523$.

31. Bae, Y. H.; Yin, H., Stability issues of polymeric micelles. Journal of controlled release 2008, 131 (1), 2-4.

32. Chen, H.; Kim, S.; Li, L.; Wang, S.; Park, K.; Cheng, J.-X., Release of hydrophobic molecules from polymer micelles into cell membranes revealed by Förster resonance energy transfer imaging. Proceedings of the National Academy of Sciences 2008, 105 (18), 6596-6601.

33. Chen, H.; Kim, S.; He, W.; Wang, H.; Low, P. S.; Park, K.; Cheng, J.-X., Fast release of lipophilic agents from circulating PEG-PDLLA micelles revealed by in vivo forster resonance energy transfer imaging. Langmuir 2008, 24 (10), 5213-5217.

34. Xu, P.; Gullotti, E.; Tong, L.; Highley, C. B.; Errabelli, D. R.; Hasan, T.; Cheng, J.-X.; Kohane, D. S.; Yeo, Y., Intracellular drug delivery by poly (lactic-co-glycolic acid) nanoparticles, revisited. Molecular pharmaceutics 2009, 6 (1), 190-201. 
35. Lavasanifar, A.; Samuel, J.; Kwon, G., Nano and microparticle drug delivery systems comprising polyesters containing aliphatic dicarboxylate residues and residues of aliphatic polyols. Adv Drug Deliv Rev 2002, 54, 169-190.

36. Gaucher, G.; Dufresne, M.-H.; Sant, V. P.; Kang, N.; Maysinger, D.; Leroux, J.-C., Block copolymer micelles: preparation, characterization and application in drug delivery. Journal of controlled release 2005, 109 (1-3), 169-188.

37. Kataoka, K.; Harada, A.; Nagasaki, Y., Block copolymer micelles for drug delivery: design, characterization and biological significance. Advanced drug delivery reviews 2001, 47 (1), 113-131.

38. Yin, H.; Kanasty, R. L.; Eltoukhy, A. A.; Vegas, A. J.; Dorkin, J. R.; Anderson, D. G., Non-viral vectors for gene-based therapy. Nature Reviews Genetics 2014, 15 (8), 541-555.

39. Lv, H.; Zhang, S.; Wang, B.; Cui, S.; Yan, J., Toxicity of cationic lipids and cationic polymers in gene delivery. Journal of Controlled Release 2006, 114 (1), 100-109.

40. Whitehead, K. A.; Langer, R.; Anderson, D. G., Knocking down barriers: advances in siRNA delivery. Nature Reviews Drug Discovery 2009, 8 (2), 129-138.

41. Abu Lila, A. S.; Ishida, T.; Kiwada, H., Recent advances in tumor vasculature targeting using liposomal drug delivery systems. Expert opinion on drug delivery 2009, 6 (12), 1297-1309.

42. Eliaz, R. E.; Nir, S.; Marty, C.; Szoka, F. C., Determination and modeling of kinetics of cancer cell killing by doxorubicin and doxorubicin encapsulated in targeted liposomes. Cancer research 2004, 64 (2), 711-718.

43. Andresen, T. L.; Jensen, S. S.; Jørgensen, K., Advanced strategies in liposomal cancer therapy: problems and prospects of active and tumor specific drug release. Progress in lipid research 2005, 44 (1), 68-97.

44. Torchilin, V. P., Recent advances with liposomes as pharmaceutical carriers. Nature reviews Drug discovery 2005, 4 (2), 145-160. 
45. Boussif, O.; Lezoualc'h, F.; Zanta, M. A.; Mergny, M. D.; Scherman, D.; Demeneix, B.; Behr, J. P., A versatile vector for gene and oligonucleotide transfer into cells in culture and in vivo: polyethylenimine. Proc Natl Acad Sci U S A 1995, 92 (16), 72977301.

46. Lungwitz, U.; Breunig, M.; Blunk, T.; Göpferich, A., Polyethylenimine-based nonviral gene delivery systems. European Journal of Pharmaceutics and Biopharmaceutics 2005, 60 (2), 247-266.

47. Wightman, L.; Kircheis, R.; Rössler, V.; Carotta, S.; Ruzicka, R.; Kursa, M.; Wagner, E., Different behavior of branched and linear polyethylenimine for gene delivery in vitro and in vivo. The Journal of Gene Medicine 2001, 3 (4), 362-372.

48. Godbey, W. T.; Wu, K. K.; Mikos, A. G., Size matters: Molecular weight affects the efficiency of poly(ethylenimine) as a gene delivery vehicle. Journal of Biomedical Materials Research 1999, 45 (3), 268-275.

49. Akinc, A.; Thomas, M.; Klibanov, A. M.; Langer, R., Exploring polyethyleniminemediated DNA transfection and the proton sponge hypothesis. The Journal of Gene Medicine 2005, 7 (5), 657-663.

50. Kichler, A.; Leborgne, C.; Coeytaux, E.; Danos, O., Polyethylenimine-mediated gene delivery: a mechanistic study. The Journal of Gene Medicine 2001, 3 (2), 135144.

51. Dufès, C.; Uchegbu, I. F.; Schätzlein, A. G., Dendrimers in gene delivery. Advanced Drug Delivery Reviews 2005, 57 (15), 2177-2202.

52. Yamagata, M.; Kawano, T.; Shiba, K.; Mori, T.; Katayama, Y.; Niidome, T., Structural advantage of dendritic poly(l-lysine) for gene delivery into cells. Bioorganic \& Medicinal Chemistry 2007, 15 (1), 526-532.

53. Lee, J. S.; Feijen, J., Polymersomes for drug delivery: design, formation and characterization. Journal of controlled release 2012, 161 (2), 473-483. 
54. Ekkelenkamp, A. E.; Elzes, M. R.; Engbersen, J. F. J.; Paulusse, J. M. J., Responsive crosslinked polymer nanogels for imaging and therapeutics delivery. Journal of Materials Chemistry B 2018, 6 (2), 210-235.

55. Graff, R. W.; Shi, Y.; Wang, X.; Gao, H., Comparison of Loading Efficiency between Hyperbranched Polymers and Cross-Linked Nanogels at Various Branching Densities. Macromolecular Rapid Communications 2015, 36 (23), 2076-2082.

56. Wang, X.; Gao, H., Recent progress on hyperbranched polymers synthesized via radical-based self-condensing vinyl polymerization. Polymers 2017, 9 (6), 188.

57. Bachelder, E. M.; Beaudette, T. T.; Broaders, K. E.; Dashe, J.; Fréchet, J. M., Acetalderivatized dextran: an acid-responsive biodegradable material for therapeutic applications. Journal of the American Chemical Society 2008, 130 (32), 1049410495.

58. Sisson, A. L.; Steinhilber, D.; Rossow, T.; Welker, P.; Licha, K.; Haag, R., Biocompatible functionalized polyglycerol microgels with cell penetrating properties. Angewandte Chemie International Edition 2009, 48 (41), 7540-7545.

59. Oh, J. K.; Siegwart, D. J.; Lee, H.-i.; Sherwood, G.; Peteanu, L.; Hollinger, J. O.; Kataoka, K.; Matyjaszewski, K., Biodegradable nanogels prepared by atom transfer radical polymerization as potential drug delivery carriers: synthesis, biodegradation, in vitro release, and bioconjugation. Journal of the American Chemical Society 2007, 129 (18), 5939-5945.

60. Gao, H.; Matyjaszewski, K., Synthesis of functional polymers with controlled architecture by CRP of monomers in the presence of cross-linkers: From stars to gels. Progress in Polymer Science 2009, 34 (4), 317-350.

61. Braunecker, W. A.; Matyjaszewski, K., Controlled/living radical polymerization: Features, developments, and perspectives. Progress in Polymer Science 2007, 32 (1), 93-146. 
62. Matyjaszewski, K.; Spanswick, J., Controlled/living radical polymerization. Materials Today 2005, 8 (3), 26-33.

63. Soni, K. S.; Desale, S. S.; Bronich, T. K., Nanogels: An overview of properties, biomedical applications and obstacles to clinical translation. Journal of Controlled Release 2016, 240, 109-126.

64. Sabir, F.; Asad, M. I.; Qindeel, M.; Afzal, I.; Dar, M. J.; Shah, K. U.; Zeb, A.; Khan, G. M.; Ahmed, N.; Din, F.-u., Polymeric nanogels as versatile nanoplatforms for biomedical applications. Journal of nanomaterials 2019, 2019.

65. Bawa, P.; Pillay, V.; Choonara, Y. E.; Du Toit, L. C., Stimuli-responsive polymers and their applications in drug delivery. Biomedical materials 2009, 4 (2), 022001.

66. Karimi, M.; Eslami, M.; Sahandi-Zangabad, P.; Mirab, F.; Farajisafiloo, N.; Shafaei, Z.; Ghosh, D.; Bozorgomid, M.; Dashkhaneh, F.; Hamblin, M. R., pH-Sensitive stimulus-responsive nanocarriers for targeted delivery of therapeutic agents. Wiley Interdisciplinary Reviews: Nanomedicine and Nanobiotechnology 2016, 8 (5), 696-716.

67. Qindeel, M.; Ahmed, N.; Sabir, F.; Khan, S.; Ur-Rehman, A., Development of novel $\mathrm{pH}$-sensitive nanoparticles loaded hydrogel for transdermal drug delivery. Drug development and industrial pharmacy 2019, 45 (4), 629-641.

68. Ramos, J.; Forcada, J.; Hidalgo-Alvarez, R., Cationic polymer nanoparticles and nanogels: from synthesis to biotechnological applications. Chemical reviews 2014, 114 (1), 367-428.

69. Stuparu, M. C.; Khan, A., Thiol-epoxy "click" chemistry: Application in preparation and postpolymerization modification of polymers. Journal of Polymer Science Part A: Polymer Chemistry 2016, 54 (19), 3057-3070.

70. Kramer, J. R.; Deming, T. J., Multimodal Switching of Conformation and Solubility in Homocysteine Derived Polypeptides. Journal of the American Chemical Society 2014, 136 (15), 5547-5550. 
71. Kramer, J. R.; Deming, T. J., Preparation of Multifunctional and Multireactive Polypeptides via Methionine Alkylation. Biomacromolecules 2012, 13 (6), 17191723.

72. Bruice, P. Y., Organic Chemistry (7th ed.). Pearson: London, 2014.

73. Park, N. H.; Fevre, M.; Voo, Z. X.; Ono, R. J.; Yang, Y. Y.; Hedrick, J. L., Expanding the Cationic Polycarbonate Platform: Attachment of Sulfonium Moieties by Postpolymerization Ring Opening of Epoxides. ACS Macro Letters 2016, 5 (11), 1247-1252.

74. Gharakhanian, E. G.; Deming, T. J., Versatile Synthesis of Stable, Functional Polypeptides via Reaction with Epoxides. Biomacromolecules 2015, 16 (6), 18021806.

75. Fröhlich, E., The role of surface charge in cellular uptake and cytotoxicity of medical nanoparticles. International journal of nanomedicine 2012, 7, 5577.

76. Ehrenberg, L.; Hussain, S., Genetic toxicity of some important epoxides. Mutation Research/Reviews in Genetic Toxicology 1981, 86 (1), 1-113.

77. Cornish, J.; Callon, K. E.; Lin, C. Q. X.; Xiao, C. L.; Mulvey, T. B.; Cooper, G. J. S.; Reid, I. R., Trifluoroacetate, a contaminant in purified proteins, inhibits proliferation of osteoblasts and chondrocytes. American Journal of PhysiologyEndocrinology and Metabolism 1999, 277 (5), E779-E783.

78. De, S.; Khan, A., Efficient synthesis of multifunctional polymersviathiol-epoxy "click" chemistry. Chemical Communications 2012, 48 (25), 3130-3132.

79. Jokerst, J. V.; Lobovkina, T.; Zare, R. N.; Gambhir, S. S., Nanoparticle PEGylation for imaging and therapy. Nanomedicine (Lond) 2011, 6 (4), 715-728.

80. Ferguson, C. J.; Hughes, R. J.; Nguyen, D.; Pham, B. T.; Gilbert, R. G.; Serelis, A. K.; Such, C. H.; Hawkett, B. S., Ab initio emulsion polymerization by RAFT-controlled self-assembly. Macromolecules 2005, 38 (6), 2191-2204. 
Chapter 4

81. Fulmer, G. R.; Miller, A. J. M.; Sherden, N. H.; Gottlieb, H. E.; Nudelman, A.; Stoltz, B. M.; Bercaw, J. E.; Goldberg, K. I., NMR Chemical Shifts of Trace Impurities: Common Laboratory Solvents, Organics, and Gases in Deuterated Solvents Relevant to the Organometallic Chemist. Organometallics 2010, 29 (9), 21762179. 


\section{Chapter 5}

\section{Sulfonium-functionalized nanogels for gene delivery}

Gene therapy is widely recognized as a promising method in combating diseases caused by gene abnormalities or deletions. Whereas most cationic carriers for gene delivery are nitrogen-based, we are interested in utilizing a sulfonium moiety to this end. Diversifying the available gene vectors not only satisfies scientific curiosity, it could also offer improved gene delivery efficiencies. Nanogels containing an epoxide moiety were subjected to post-synthesis modifications resulting in biocompatible sulfonium nanogels containing a thioglycerol moiety. These hydrophilic nanogels were successfully incubated with plasmid DNA at various ratios. Characterization of the resulting polyplexes indicated a promising start in the field of sulfonium nanogels for gene therapy, as well as various points of interest requiring optimization and further research.
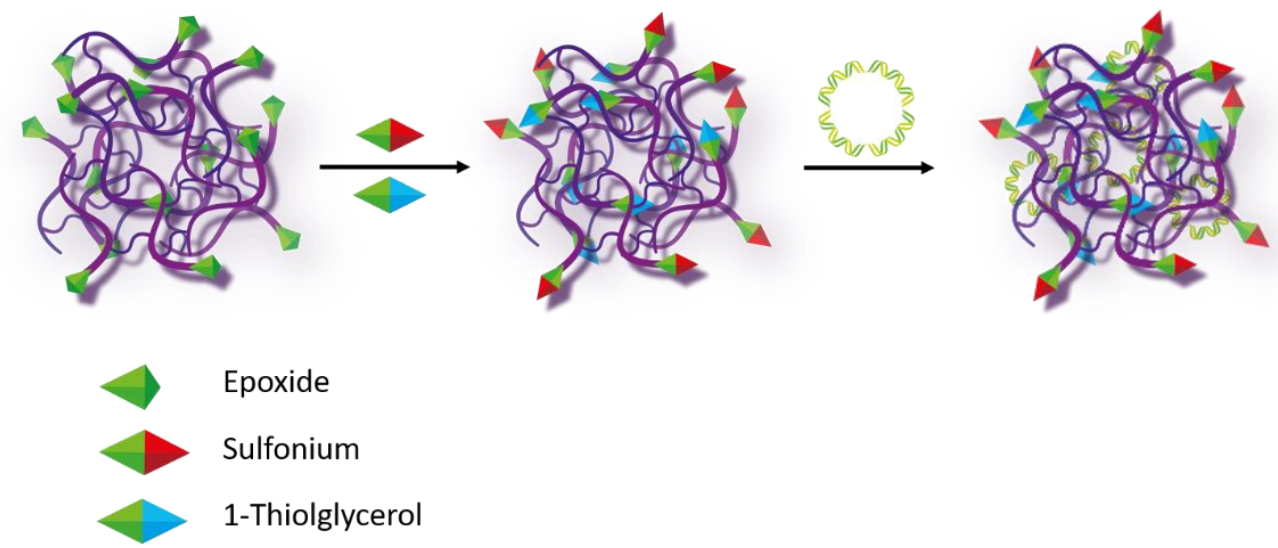

1-Thiolglycero 


\subsection{Introduction}

In recent decades, the use of nanotechnology for biomedical applications has risen rapidly; for example with drug and gene delivery systems, imaging and diagnostic agents, and implantable materials. ${ }^{1-2}$ This field, known as nanomedicine, exploits the unique properties (e.g. optical, electronic) of nanoscale materials.

\subsubsection{Sulfonium versus ammonium moiety in gene delivery}

Gene therapy is a branch of nanomedicine in which foreign genes are introduced into the human body with the aim to cure diseases, by stimulating or downregulating certain gene expressions. ${ }^{3-4}$ Nanoscale delivery vehicles are used to protect, transport, mediate cellular uptake and endosomal release of genetic material such as nucleic acids. ${ }^{5}$ As both nucleic acids and cell membranes are negatively charged, cellular uptake of free nucleic acid is limited. Gene delivery vehicles can be viral macromolecules or nonviral polymer nanoparticles. ${ }^{6}$ However, as viral macromolecules present many limitations, polymeric vectors are preferred. ${ }^{3}$ Cationic polymers can efficiently bind negatively charged genetic material, forming so-called polyplexes. ${ }^{7-8}$ These positively charged polymers mediate cellular uptake by interacting with the cell membrane through multivalent interactions. Subsequent endosomal escape is facilitated by the cationic polymer through their buffering capacity and proton sponge effect. ${ }^{9-10}$

Until recently, most polymeric gene delivery systems were nitrogen-based cationic systems such as poly-L-lysine, chitosan, Superfect and PEI. ${ }^{11-17}$ Although these nitrogen-based systems are capable of achieving high transfection efficiency, many also exhibit unwanted high cytotoxicity. Efforts have been made to lower the accompanying cytotoxicity by varying the degree of amine substitution, by introducing PEG substituents or by tuning the $\mathrm{pK}_{\mathrm{a}}$ of the amine. In 2012, Hemp et al. first showed nucleic acid delivery 
using phosphonium-based cationic macromolecules as an alternative to ammonium macromolecules. ${ }^{18}$ These materials have proven to be suitable alternatives to ammoniumbased cationic carrier materials, with reports of lower cytotoxicity and higher transfection efficiency. ${ }^{19-20}$ Furthermore, phosphonium based polymers were reported to have higher binding affinities with nucleic acids, possibly due to the centered charge and larger atom size. ${ }^{18,21}$ Another alternative to nitrogen-based charge carriers is the sulfonium group, based on cationic tricoordinate organosulfur compounds. Sulfonium groups have a lone pair of electrons, three substituents and a tetrahedral geometry (like quaternary ammonium groups). In a study by Kurnia et al. the charge distribution of sulfonium ions was compared with ammonium ions. ${ }^{22}$ The $S$ atom in triethylsulfonium was calculated to have a charge of $+0.9 \mathrm{e}$, whereas the $\mathrm{N}$ atom of butyltrimethylammonium possesses a charge of -0.3 e. This means that the positive charge of the sulfonium ion is more centered compared to the ammonium ion. Furthermore, sulfur has an ionic radius of $170 \mathrm{pm}$, compared to an ionic radius of $132 \mathrm{pm}$ for nitrogen..$^{23}$ As such, the $\mathrm{S}^{+}$atom in sulfonium groups has a larger radius and a more centered charge compared to $\mathrm{N}^{+}$in ammonium, possibly offering increased nucleic acid binding efficiencies.

Hemp et al. first reported on sulfonium based polymers for gene transfection in 2013, demonstrating successful complexation and delivery of nucleic acids in vitro (Figure 1).$^{24}$ Furthermore, Mackenzie et al. reported on sulfonium functionalized polymers, that were shown to be biocompatible, stable towards dealkylation, and able to successfully complex and deliver short interfering RNA in vitro. ${ }^{25}$ Zhu et al. designed intracellularly disintegrable polysulfonium compounds, which were able to effectively condense DNA into polyplexes and achieve high transfection effeciency. ${ }^{26}$ Although the field of sulfonium-based polymers for gene delivery is relatively young, the results are quite promising. So far, exclusively linear polysulfonium vectors have been explored as gene carriers. Incorporating sulfonium groups into three-dimensional polymer architectures 
such as nanogels might offer new insights into the complexation of DNA and transfection by sulfonium-based charge carriers.

a)<smiles>C[S+](C)CCOC(=O)C(C)(CC(C)(C)C)C(C)(C)C</smiles>

$\mathrm{Cl}^{-}$

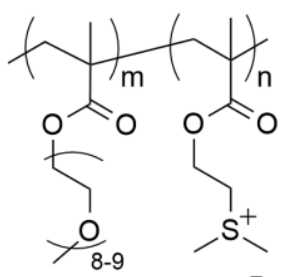

$\mathrm{Cl}^{-}$ b)

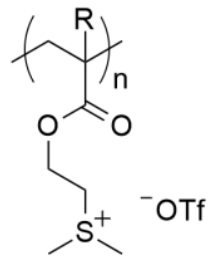

$\mathrm{R}=\mathrm{H}, \mathrm{CH}_{3}$ c)<smiles>[R]C[S+](C)Cc1cccc(CC(C)(C)C)c1B1OC(C)(C)C(C)(C)O1</smiles>

$\mathrm{R}=\mathrm{C}_{2} \mathrm{H}_{4}, \mathrm{C}_{4} \mathrm{H}_{8}$ or $\mathrm{CH}_{2} \mathrm{OC}_{2} \mathrm{H}_{4} \mathrm{OCH}_{2}$

Figure 1. Overview of polysulfonium structures for gene delivery, as reported by a) Hemp et al. ${ }^{24}$, b) Mackenzie et al., ${ }^{25}$ and c) Zhu et al. ${ }^{27}$.

As others achieved great success transfecting cells with amine derivatives of poly(glycidyl methacrylate) (pGMA), we attempted GFP gene transfection with sulfonium GMA nanogels. ${ }^{28-31}$ In this chapter the construction and characterization of polyplexes between sulfonium nanogels and plasmid DNA encoding for green fluorescent protein (pCMV-GFP) is reported. Their application in gene transfection is discussed in chapter 6 of this thesis.

\subsection{Results and Discussion}

\subsubsection{Sulfonium nanogel synthesis}

GMA and TTEGDMA were polymerized in a RAFT polymerization at $9 \mathrm{w} / \mathrm{w} \%$ in EtOAc forming GMA-TTEGDMA nanogels bearing a pendant epoxide moiety (see chapter 4). 

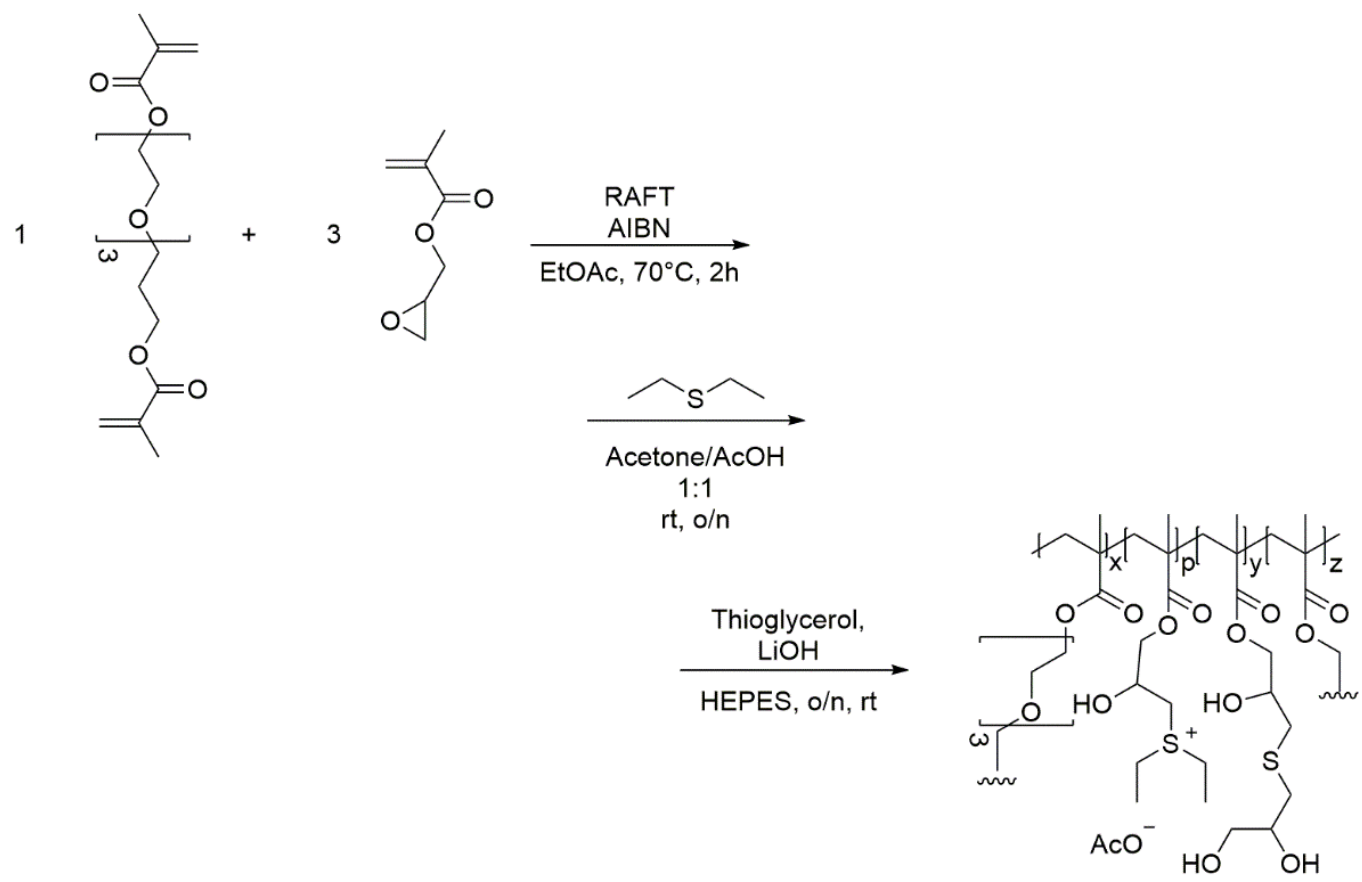

Scheme 1. The nanogel structure is depicted in its simplified version for clarity purposes. Thioglycerol reacts with the epoxides was well as with the pendent remaining methacrylates via a thiol-Michael addition (not pictured).

DLS analysis gave a hydrodynamic diameter of $24 \mathrm{~nm}$ in DCM. ${ }^{1} \mathrm{H}$ NMR spectroscopy indicated a successful reaction displaying epoxide signals at $\delta=2.6 \mathrm{ppm}, \delta=2.8 \mathrm{ppm}$ and $\delta=3.2 \mathrm{ppm}$. In a follow up reaction, diethyl sulfide was reacted with the epoxide groups under acidic conditions forming GMA-TTEGDMA-S+-40 and GMA-TTEGDMA-S+-50 with 40 and 50 indicating the epoxide conversion. GMA-EGDMA-S+-15 and GMA-TTEGDMA-S+36 were also formed as it was our intention to compare sulfonium nanogels with various surface charges. However, these nanogels decomposed and/or hydrolyzed during handling and purification. 


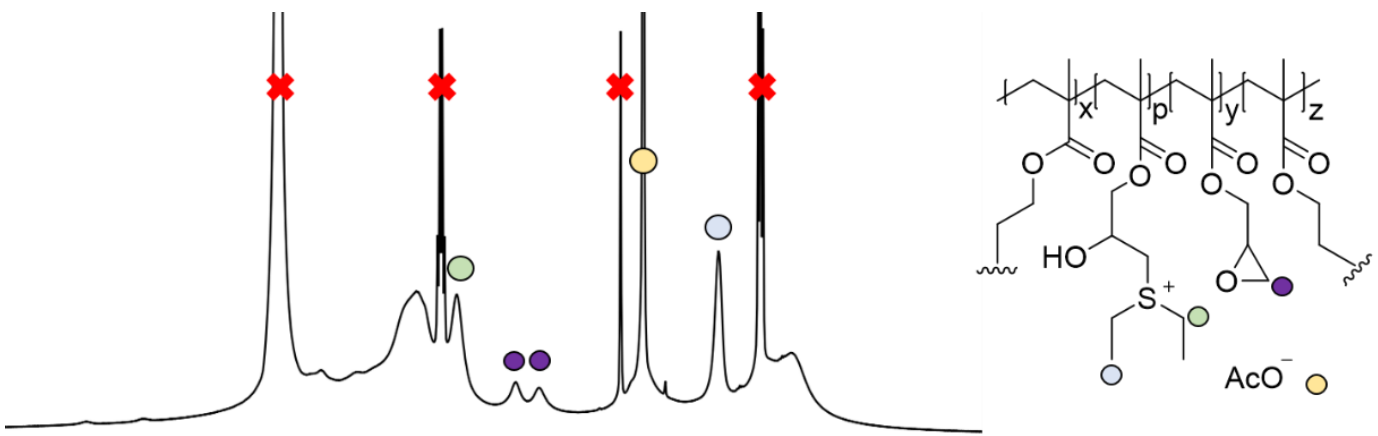

$\begin{array}{llllllllllllll}6.5 & 6.0 & 5.5 & 5.0 & 4.5 & 4.0 & 3.5 & 3.0 & 2.5 & 2.0 & 1.5 & 1.0 & 0.5 & 0.0\end{array}$

Chemical shift (ppm)

Figure 2. ${ }^{1} \mathrm{H}$ NMR spectrum of GMA-TTEGDMA-S+-50 displaying the remaining epoxide peaks at $\delta=2.6 \mathrm{ppm}$ and $\delta=2.8 \mathrm{ppm}$ (purple), the sulfonium methylene groups at $\delta=3.5 \mathrm{ppm}$ (green) and the methyl groups at $\delta$ $=1.4 \mathrm{ppm}$ (blue). The acetate counter ion is seen at $\delta=2.1 \mathrm{ppm}$ (yellow).

The hydrodynamic diameter and surface charge was measured for both nanogels in 10 $\mathrm{mM}$ HEPES at $\mathrm{pH}=7$. GMA-TTEGDMA-S ${ }^{+}-50$ was $27 \mathrm{~nm}$ in diameter with a zeta potential of $+31 \mathrm{mV}$. TTEGDMA-S $\mathrm{s}^{+}-40$ was $67 \mathrm{~nm}$ in diameter and had a surface charge of $+29 \mathrm{mV}$. To enhance the biocompatibility of these nanogels they were reacted with 1-thioglycerol to GMA-TTEGDMA-S ${ }^{+}$-40-THG (40-THG) and GMA-TTEGDMA-S ${ }^{+}-50-$ THG (50-THG), quenching the remaining epoxides. This reaction was carried out under basic conditions in HEPES solutions. DLS measurements gave a hydrodynamic diameter of $39 \mathrm{~nm}$ in $10 \mathrm{mM}$ HEPES buffer $(2 \mathrm{mg} / \mathrm{mL})$ and a surface charge of $+9 \mathrm{mV}$ at $0.5 \mathrm{mg} / \mathrm{mL}$ for 40-THG. 50-THG had a diameter of $24 \mathrm{~nm}$ and a surface charge of $+14 \mathrm{mV}$. The decrease in surface charge is likely an indication of the stability of the sulfonium charge on these nanogels. NMR analysis of 40-THG and 50-THG display a decrease in the intensity of the acetate counter ion peak, likely indicating hydrolysis of the sulfonium moiety. Surprisingly others observed minimal dealkylation of polysulfonium polymers in aqueous environment. ${ }^{25}$ 
To further analyze the properties of these nanogels their infrared (IR) absorptions were measured. The epoxide stretch at $907 \mathrm{~cm}^{-1}$ is no longer present and new peaks arise for $\mathrm{S}^{+}-\mathrm{C}$ vibrational stretches at $585 \mathrm{~cm}^{-1}$ and $632 \mathrm{~cm}^{-1}$ as seen in Figure 3 . Analysis of the nanogels by TEM demonstrated spherical particles with a high polydispersity. Due to low quantities of 40-THG TEM micrographs had to be made at highly dilute concentrations. As such it was not possible to accurately calculate the mean particle diameter.

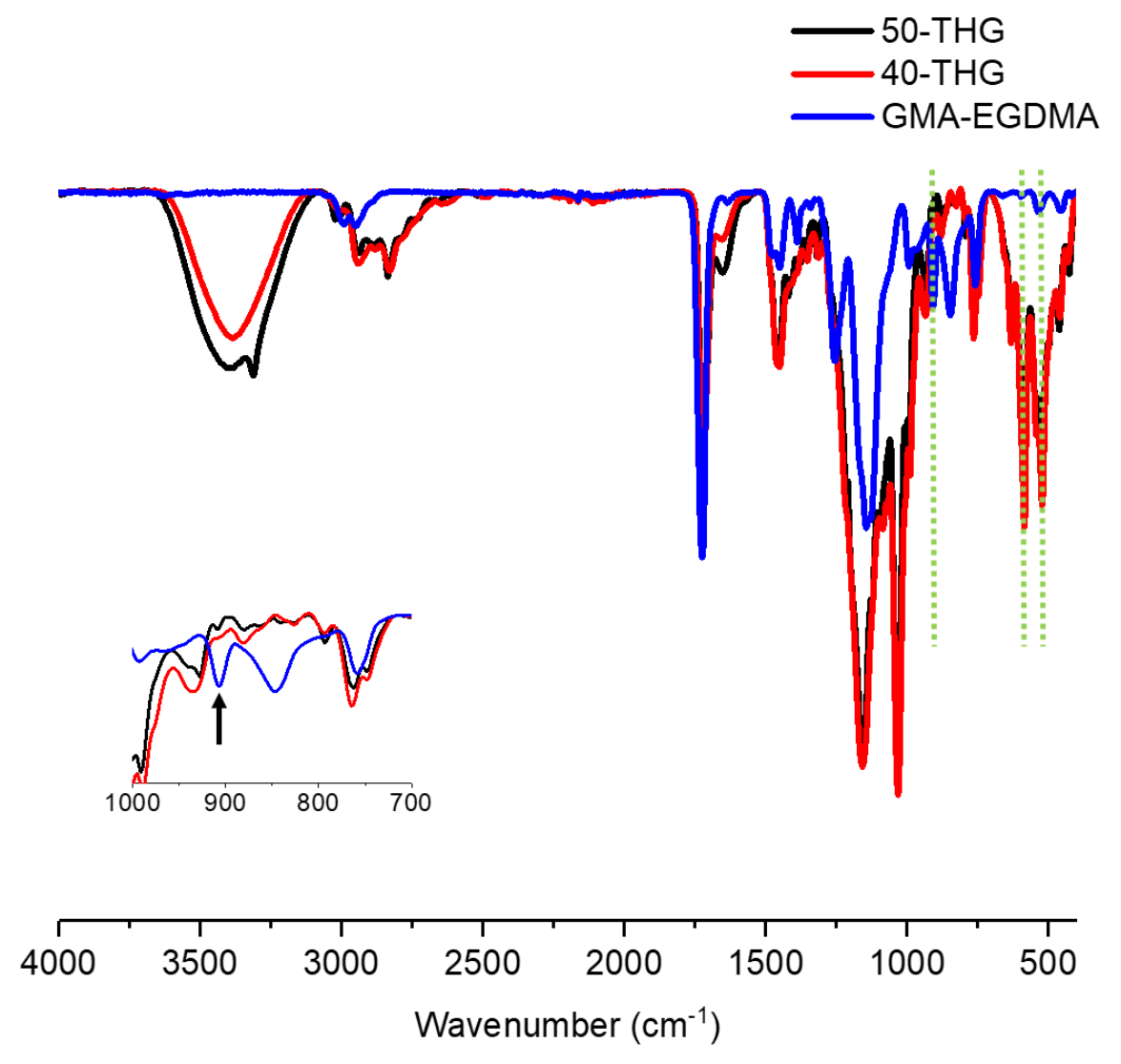

Figure 3. Green dotted lines over peaks at $v=585 \mathrm{~cm}^{-1}$ and $v=632 \mathrm{~cm}^{-1}$ highlight the emergence of $\mathrm{S}^{+}-\mathrm{C}$ vibrational stretches. The green dotted line (and arrow in the inset) at $v=907 \mathrm{~cm}^{-1}$ specifies the disappearance of epoxide moieties demonstrating a successful reaction. 

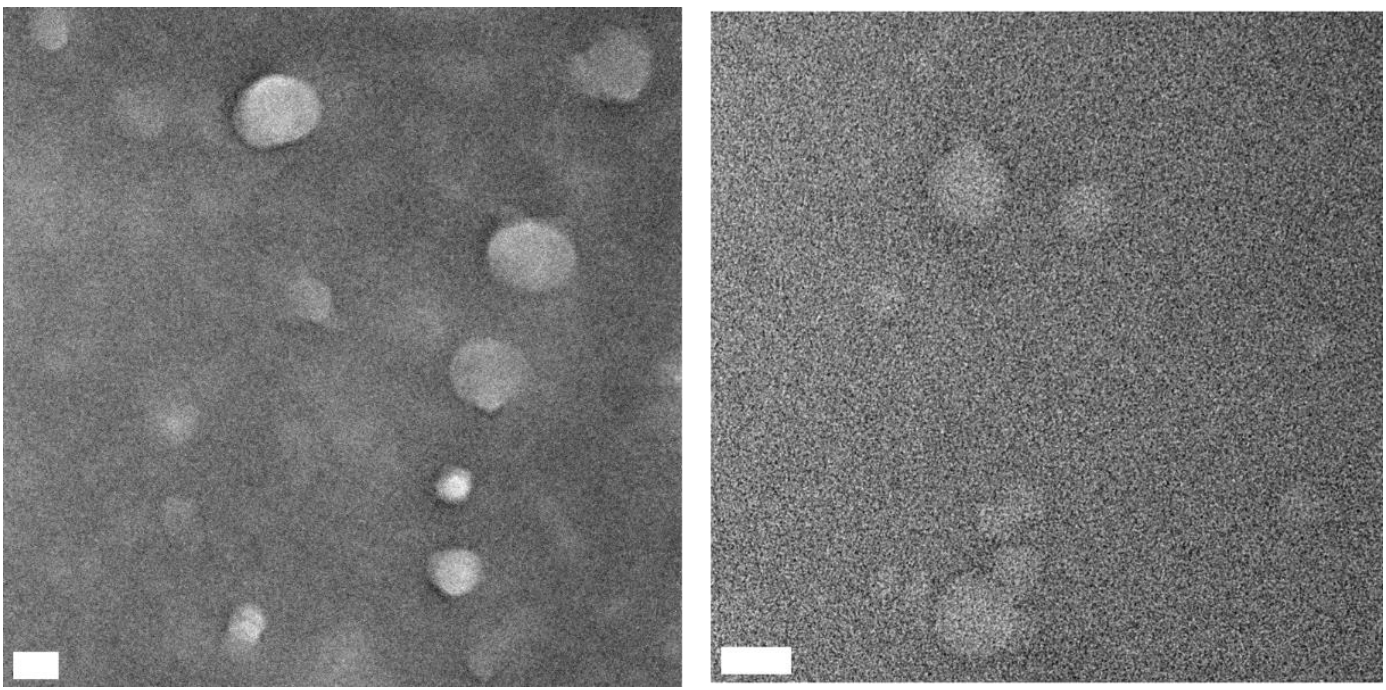

Figure 4. TEM images of 50-THG (left) and 40-THG (right) indicate the formation of spherical particles with high polydispersity. The scale bars represent $50 \mathrm{~nm}$. 40-THG had a particle diameter of $79 \pm 56 \mathrm{~nm}$ and $\mathbf{5 0}$ THG had a particle diameter of $61 \pm 17 \mathrm{~nm}$.

\subsubsection{Polyplex formation with sulfonium nanogels}

The complexation abilities of the nanogels with plasmid DNA were studied utilizing plasmid DNA encoding for green fluorescent protein (pCMV-GFP). Polyplexes were formed at various S/P ratios and their size and surface charge were measured. S/P ratio refers to the ratio between the estimated $\mathrm{S}^{+}$atoms in the nanogel and the phosphate units in the plasmid DNA. As the DNA and cationic nanogel bind tighter to each other due to electrostatic interactions, the size of the polyplexes decreases at higher S/P ratios until maximum polyplex density is achieved. 50-THG reached a maximum density and minimum hydrodynamic diameter at $\mathrm{S} / \mathrm{P}=50$ as can be seen in Figure 5. This plateau indicates the formation of stable polyplexes. A similar trend is seen for the surface charge of the polyplexes. The positively charged nanogel will complex and shield the DNA and its negative charge resulting in an increase in surface charge which eventually stagnates. 
The complexation abilities of 40-THG were studied in a similar manner. Polyplexes were formed at various S/P ratios and their hydrodynamic diameter and surface charge were measured. 40-THG required higher S/P ratios to form tight polyplexes. The eventual plateau in size was less pronounced for 40-THG than for 50-THG, and not at all present for the surface charge. Most likely 40-THG forms polyplexes with lower binding affinity between nanogel and pDNA, leading to weaker electrostatic interactions than 50-THG. As a result the polyplexes formed are less stable and not always quantifiable (e.g. the surface charge of $S / P=125$ ).
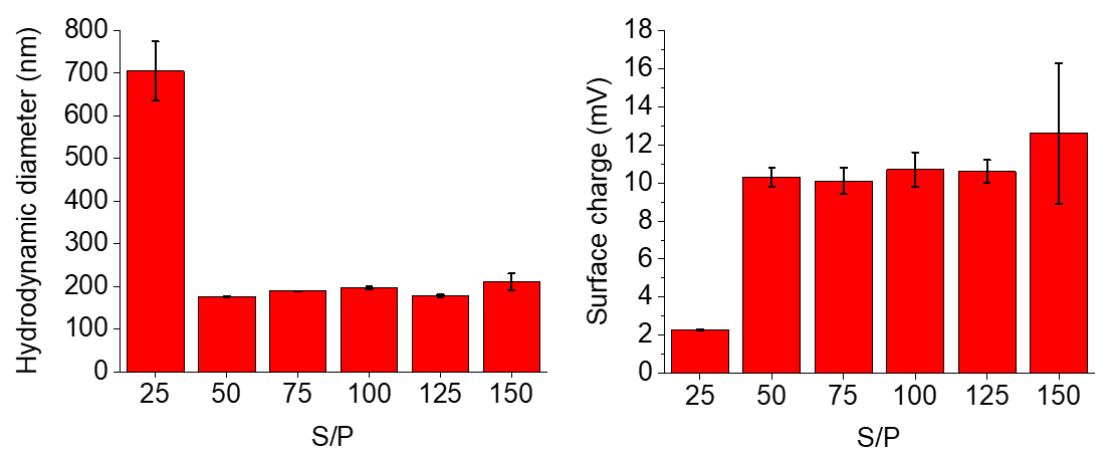

Figure 5. DLS and zeta potential measurements of 50-THG polyplexes at various S/P ratios indicate proper polyplex formation starting from $\mathrm{S} / \mathrm{P}=50$ in $10 \mathrm{mM}$ HEPES.
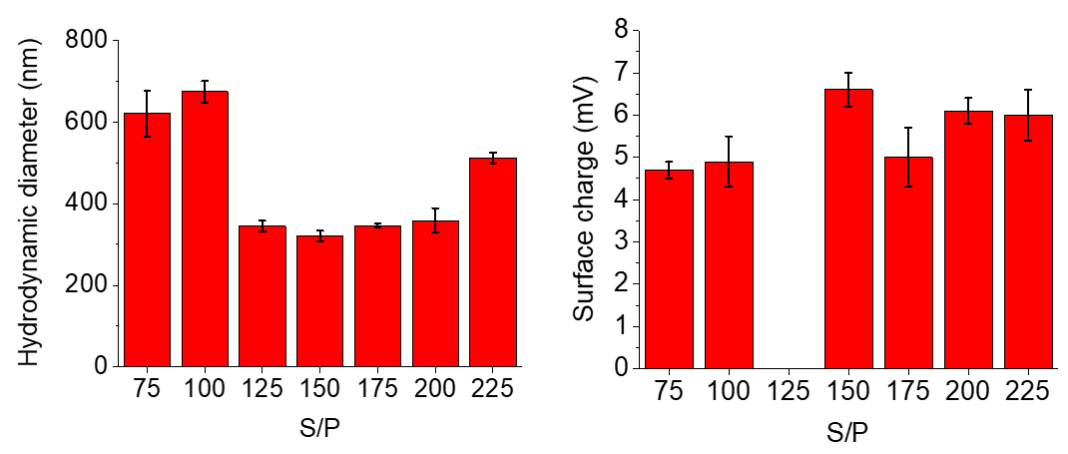

Figure 6. DLS and zeta potential measurements of 40-THG indicate that much higher S/P ratios were required to achieve compact polyplexes compared to $\mathbf{5 0 - T H G . ~}$ 
The differences between the two sets of polyplexes are likely due to the different properties of the nanogels. 40-THG has a lower surface charge ( $+9 \mathrm{mV}$ compared to +14 $\mathrm{mV}$ for 50-THG), requiring higher amounts of nanogels (and thus higher S/P ratios) to form adequate polyplexes. Higher numbers of $39 \mathrm{~nm}$ nanogels directly translates to larger polyplexes (Figure 6) as well as decreased stability - one negative charge has to interact with increasingly more bulky positive charges.

The ideal size of particles for in vitro studies are smaller than $200 \mathrm{~nm}$ to promote cellular uptake through endocytosis. ${ }^{32-34}$ As such it can be concluded that 40-THG is not suitable for in vitro transfection experiments. Optimizing the sulfonium nanogel properties, such as increasing the surface charge could result in stable polyplexes at lower $\mathrm{S} / \mathrm{P}$ ratios. However, a higher charge density is expected to cause a size increase as sulfonium moieties will repel each other, leading to polyplexes larger than the desired 200 $\mathrm{nm}$. This trade-off can be eased by starting with smaller GMA-TTEGDMA epoxide nanogels. It is worth noting however, that this strategy cannot be utilized to its full extent as high positive charge has been proven to be toxic to cells ${ }^{35}$, meaning there is an upper limit to the cationic charge of the nanogels/polyplexes.

\subsubsection{Polyplex stability}

In order to quantify the stability of 50-THG polyplexes their hydrodynamic diameter and surface charge were studied for an extended period of time. Polyplexes were formed at $\mathrm{S} / \mathrm{P}=75$ and their properties were measured every $60 \mathrm{~min}$. The electrostatic interactions that form the polyplexes start dissociating, releasing the negatively charged DNA that is inside. As a result these polyplexes become less dense and increase in size while decreasing in overall surface charge. Starting at $3 \mathrm{~h}$ significant changes are seen. 

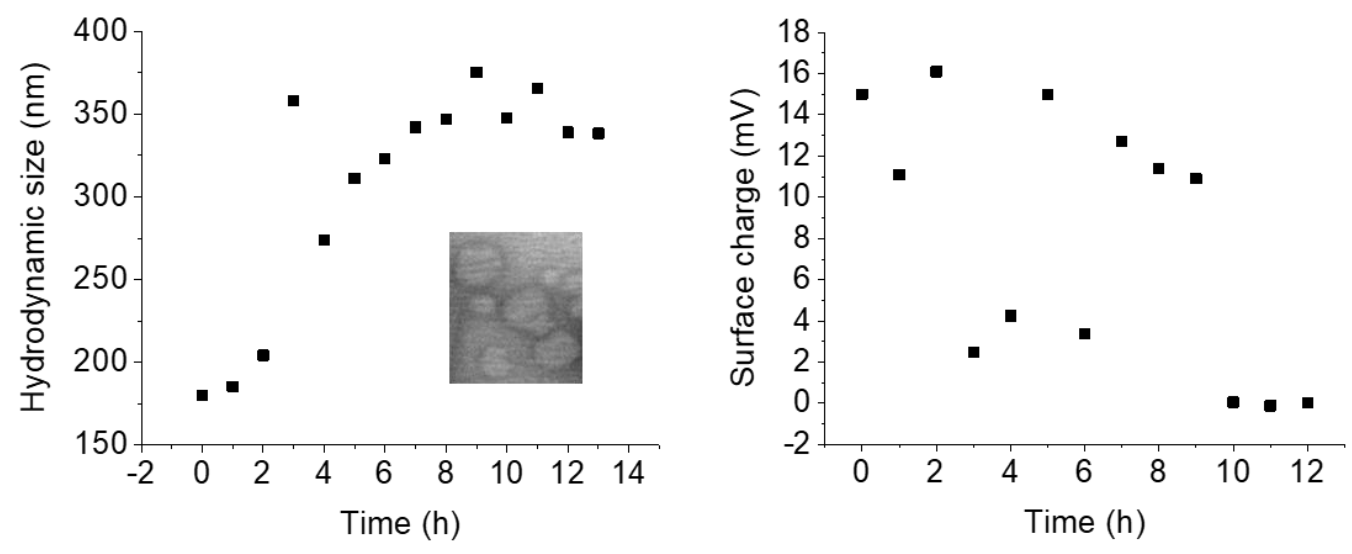

Figure 7. DLS and zeta potential measurements of 50-THG polyplexes with pCMV-GFP indicate that the polyplexes formed are stable for $3 \mathrm{~h}$, after which their bonds start dissociating. This is mirrored in the size increase and charge decrease. Inset: TEM micrograph at $S / P=75$.

\subsection{Conclusions}

In this work we synthesized two sulfonium nanogels with a thioglycerol moiety from an epoxide containing precursor. The efficiency of these nanogels at forming polyplexes with negatively charged plasmid DNA was evaluated, resulting in 50-THG outperforming 40THG. The polyplexes formed from 40 -THG have a low surface charge of $+6 \mathrm{mV}$, an average size of $300 \mathrm{~nm}$ and limited stability. These properties are less than favorable to future transfection studies. 50-THG on the contrary forms stable polyplexes starting from S/P = 50, with an average size of $150 \mathrm{~nm}$ and an average surface charge of $+10 \mathrm{mV}$. These polyplexes are also stable up to $3 \mathrm{~h}$. As such it can be concluded that 50-THG is better suited for in vitro transfection studies. It can also be concluded that, for this system, polyplexes (and their nanogel precursors) have a minimum surface charge requirement in order to be eligible for in vitro studies. Repeating this study at various surface charge 
densities would provide valuable insight. A better understanding of the stability of the sulfonium moiety is also crucial in efforts to optimize polyplex formation.

\subsection{Acknowledgements}

Dr. E. G. Keim is acknowledged for his help with TEM measurements. Rachèl Elzes and Naomi Hamelmann are thanked for their help and guidance.

\subsection{Experimental section}

Materials. Glycidyl methacrylate (GMA, 97\%) and ethylene glycol dimethacrylate (EGDMA, 98\%), tetraethylene glycol dimethacrylate (TTEGDMA, >90\%), were purchased from Sigma-Aldrich and passed through a column of neutral $\mathrm{Al}_{2} \mathrm{O}_{3}$ prior to use. HEPES sodium salt (99.5\%), N,N-dimethylformamide (DMF, anhydrous, 99.8\%), sodium hydroxide pellets $(\mathrm{NaOH})$, azobisisobutyronitrile (AIBN, 98\%) and lithium hydroxide ( $\mathrm{LiOH}$, reagent grade, 98\%) were also purchased from Sigma-Aldrich and used without further purification. Acetic acid glacial $(\mathrm{AcOH})$ was obtained from VWR chemicals. Dichloromethane (DCM, 99.7\%), acetone (99.5\%) and ethylacetate (EtOAc, 99.5\%) were obtained from Ossum Chemicals. The RAFT agent 2-[[(butylthio)thioxomethyl] thio] propanoic acid was synthesized following a literature procedure. ${ }^{36}$ Plasmid DNA (pCMVGFP) was purchased from the Plasmid Factory. Deuterated solvents were purchased from Sigma-Aldrich and used as received. The water used in this work was treated through a Milli-Q Gradient System. Dialysis was done using Spectra/Por 6 Dialysis Membrane Prewetted RC Tubing with a molecular weight cut-off of $1 \mathrm{kDa}$. 
Analyses. Infrared spectroscopy (FT-IR) was performed on a Bruker Alpha and analyzed with OPUS software. DLS and zeta potential measurements were carried out on a Malvern Zetasizer 4000 , performed in triplicate. As a reference material for DLS, poly(ethylene glycol)-co-poly(isobutyl methacrylate) with a refractive index (RI) of 1.465 was used. DLS measurements were carried with concentrations in the range of 0.5-2.0 $\mathrm{mg} / \mathrm{mL}$. Zeta potential measurements were carried out at similar concentrations in $10 \mathrm{mM}$ HEPES buffer solution (HEPES-NaOH, pH = 7). TEM was carried out on a Philips CM300STFEG Transmission Electron Microscope. Samples were prepared on Electron Microscopy Sciences FCF200-Cu Formvar/copper support grids (200 mesh). Solutions of nanogels were prepared with concentrations of $1 \mathrm{mg} / \mathrm{mL}$ in $10 \mathrm{mM}$ HEPES and sonicated for $30 \mathrm{~min}$. Of this solution $5 \mu \mathrm{L}$ was deposited on the grid and dried after 15 min with paper filter. After drying of the grid in the fume hood, $5 \mu \mathrm{L}$ of an aqueous $1 \% \mathrm{w} / \mathrm{v}$ uranyl acetate solution was deposited on the grid and dried after 30 seconds, using a paper filter. Particle size analysis using TEM micrographs was carried out using ImageJ software by calibrating to the scale given in the micrographs.

GMA-TTEGDMA nanogel formation. RAFT agent 2-[[(butylthio)thioxomethyl] thio] propanoic acid ( $337.4 \mathrm{mg}, 1.4 \mathrm{mmol}, 1$ equiv.), AIBN ( $46.5 \mathrm{mg}, 0.3 \mathrm{mmol}, 0.2$ equiv.), GMA (7 mL, 53 mmol, 37.5 equiv.), TTEGDMA ( $5.4 \mathrm{~mL}, 17.7 \mathrm{mmol}, 12.5$ equiv.), DMF (4 $\mathrm{mL}, 53 \mathrm{mmol}, 37.5$ equiv.) as internal standard and EtOAc ( $150 \mathrm{~mL}, 91 \mathrm{w} / \mathrm{w} \%)$ were added to a $250 \mathrm{~mL}$ round bottom flask equipped with a stir bar, sealed with a septum and purged with nitrogen for $20 \mathrm{~min}$ in an ice-salt bath. The flask was subsequently placed in an oil bath at $70{ }^{\circ} \mathrm{C}$ and allowed to react for $2 \mathrm{~h}$ after which it was taken out of the oil bath and quenched with $12 \mathrm{~mL}$ DCM. The nanogel was precipitated three times in hexane yielding a white solid (1.9 g, 17\% conversion, $4.59 \mathrm{mmol} / \mathrm{g}$ epoxides).

GMA-TTEGDMA-S+-x nanogel formation. In a typical procedure, GMA-TTEGDMA (245.5 mg, $1.13 \mathrm{mmol}$ of epoxides, 1 equiv.) was dissolved in a mixture of $\mathrm{AcOH} /$ acetone 
(1:1, $25 \mathrm{~mL})$, followed by addition of diethyl sulfide (1.2 mL, $11.3 \mathrm{mmol}, 10$ equiv.). After stirring for $2 \mathrm{~h}$ at room temperature, the nanogel was precipitated three times in diethyl ether and dissolved in $10 \mathrm{mM}$ HEPES (HEPES- $\mathrm{NaOH}, \mathrm{pH}=7$ ) obtaining a solution of GMAEGDMA-S $\mathbf{S}^{+}-\mathbf{x}$.

GMA-TTEGDMA-S+-x-THG. In a typical procedure, to a snap cap vial containing GMA-TTEGDMA-S $\mathbf{S}^{+} \mathbf{x}(81 \mathrm{mg}, 0.37 \mathrm{mmol}$ of epoxides, 1.0 equiv.) in $10 \mathrm{mM}$ HEPES (HEPES$\mathrm{NaOH}, \mathrm{pH}=7$ ) solution was added, $\mathrm{LiOH}(26.5 \mathrm{mg}, 1.1 \mathrm{mmol}, 3$ equiv.) and 1-thioglycerol ( $0.1 \mathrm{~mL}, 1.1 \mathrm{mmol}, 3$ equiv.) and was allowed to stir overnight at room temperature. The reaction mixture was then dialyzed $(\mathrm{MWCO}=1 \mathrm{kDa})$ for $2 \mathrm{~d}$ against water. HEPES salt and $\mathrm{NaOH}$ were added to the aqueous solution to obtain GMA-TTEGDMA-S+-x-THG $10 \mathrm{mM}$ HEPES ( 3.3 or $2 \mathrm{mg} / \mathrm{mL}, \mathrm{HEPES}-\mathrm{NaOH}, \mathrm{pH}=7$ ).

Polyplex preparation. In a typical procedure, GMA-TTEGDMA-S+-x-THG in $10 \mathrm{mM}$ HEPES ( 3.3 or $2 \mathrm{mg} / \mathrm{mL}$, HEPES- $\mathrm{NaOH}, \mathrm{pH}=7$ ) was sterilized by means of filtration with a $200 \mathrm{~nm}$ syringe filter to form a stock solution. Various amounts of this stock solution, 10 mM HEPES buffer (HEPES-NaOH, $\mathrm{pH}=7$ ) and plasmid DNA were combined to form polyplexes with S/P ratios of 50 to 225 . The S/P ratio is the molar ratio between the sulfonium $(\mathrm{S})$ atoms of the nanogel and the phosphate $(\mathrm{P})$ atoms of the plasmid DNA. The polyplexes were incubated at RT for $10 \mathrm{~min}$ before measuring the hydrodynamic diameters and surface charges on a Malvern Nano Zetasizer in triplicate.

\subsection{References}

1. Chen, G.; Roy, I.; Yang, C.; Prasad, P. N., Nanochemistry and Nanomedicine for Nanoparticle-based Diagnostics and Therapy. Chemical Reviews 2016, 116 (5), 2826-2885. 
2. Etheridge, M. L.; Campbell, S. A.; Erdman, A. G.; Haynes, C. L.; Wolf, S. M.; McCullough, J., The big picture on nanomedicine: the state of investigational and approved nanomedicine products. Nanomedicine: Nanotechnology, Biology and Medicine 2013, 9 (1), 1-14.

3. Luo, D.; Saltzman, W. M., Synthetic DNA delivery systems. Nature biotechnology 2000, 18 (1), 33-37.

4. Tatiparti, K.; Sau, S.; Kashaw, S. K.; Iyer, A. K., siRNA delivery strategies: a comprehensive review of recent developments. Nanomaterials 2017, 7 (4), 77.

5. Mintzer, M. A.; Simanek, E. E., Nonviral vectors for gene delivery. Chemical reviews 2009, 109 (2), 259-302.

6. Ogawara, K.-I.; Yoshida, M.; Furumoto, K.; Takakura, Y.; Hashida, M.; Higaki, K.; Kimura, T., Uptake by hepatocytes and biliary excretion of intravenously administered polystyrene microspheres in rats. Journal of drug targeting 1999, 7 (3), 213-221.

7. Mammen, M.; Choi, S. K.; Whitesides, G. M., Polyvalent interactions in biological systems: implications for design and use of multivalent ligands and inhibitors. Angewandte Chemie International Edition 1998, 37 (20), 2754-2794.

8. Fasting, C.; Schalley, C. A.; Weber, M.; Seitz, O.; Hecht, S.; Koksch, B.; Dernedde, J.; Graf, C.; Knapp, E. W.; Haag, R., Multivalency as a chemical organization and action principle. Angewandte Chemie International Edition 2012, 51 (42), 1047210498.

9. Sonawane, N.; Szoka, F. C.; Verkman, A., Chloride accumulation and swelling in endosomes enhances DNA transfer by polyamine-DNA polyplexes. Journal of Biological Chemistry 2003, 278 (45), 44826-44831.

10. Edinger, D.; Wagner, E., Bioresponsive polymers for the delivery of therapeutic nucleic acids. Wiley interdisciplinary reviews: nanomedicine and nanobiotechnology 2011, 3 (1), 33-46. 
11. Boussif, O.; Lezoualc'h, F.; Zanta, M. A.; Mergny, M. D.; Scherman, D.; Demeneix, B.; Behr, J. P., A versatile vector for gene and oligonucleotide transfer into cells in culture and in vivo: polyethylenimine. Proc Natl Acad Sci U S A 1995, 92 (16), 72977301.

12. Parhamifar, L.; Larsen, A. K.; Hunter, A. C.; Andresen, T. L.; Moghimi, S. M., Polycation cytotoxicity: a delicate matter for nucleic acid therapy-focus on polyethylenimine. Soft Matter 2010, 6 (17), 4001-4009.

13. Godbey, W. T.; Wu, K. K.; Mikos, A. G., Poly(ethylenimine) and its role in gene delivery. Journal of Controlled Release 1999, 60 (2), 149-160.

14. Köping-Höggård, M.; Tubulekas, I.; Guan, H.; Edwards, K.; Nilsson, M.; Vårum, K. M.; Artursson, P., Chitosan as a nonviral gene delivery system. Structure-property relationships and characteristics compared with polyethylenimine in vitro and after lung administration in vivo. Gene therapy 2001, 8 (14), 1108-1121.

15. Strand, S. P.; Lelu, S.; Reitan, N. K.; de Lange Davies, C.; Artursson, P.; Vårum, K. M., Molecular design of chitosan gene delivery systems with an optimized balance between polyplex stability and polyplex unpacking. Biomaterials 2010, 31 (5), 975-987.

16. Luo, D.; Haverstick, K.; Belcheva, N.; Han, E.; Saltzman, W. M., Poly (ethylene glycol)-conjugated PAMAM dendrimer for biocompatible, high-efficiency DNA delivery. Macromolecules 2002, 35 (9), 3456-3462.

17. Dufès, C.; Uchegbu, I. F.; Schätzlein, A. G., Dendrimers in gene delivery. Advanced drug delivery reviews 2005, 57 (15), 2177-2202.

18. Hemp, S. T.; Allen, M. H.; Green, M. D.; Long, T. E., Phosphonium-Containing Polyelectrolytes for Nonviral Gene Delivery. Biomacromolecules 2012, 13 (1), 231238. 
19. Ornelas-Megiatto, C. t.; Wich, P. R.; Fréchet, J. M., Polyphosphonium polymers for siRNA delivery: an efficient and nontoxic alternative to polyammonium carriers. Journal of the American Chemical Society 2012, 134 (4), 1902-1905.

20. Rose, V. L.; Mastrotto, F.; Mantovani, G., Phosphonium polymers for gene delivery. Polymer Chemistry 2017, 8 (2), 353-360.

21. Ornelas-Megiatto, C.; Wich, P. R.; Fréchet, J. M. J., Polyphosphonium Polymers for siRNA Delivery: An Efficient and Nontoxic Alternative to Polyammonium Carriers. Journal of the American Chemical Society 2012, 134 (4), 1902-1905.

22. Kurnia, K. A.; Quental, M. V.; Santos, L. M. N. B. F.; Freire, M. G.; Coutinho, J. A. P., Mutual solubilities between water and non-aromatic sulfonium-, ammonium- and phosphonium-hydrophobic ionic liquids. Physical Chemistry Chemical Physics 2015, 17 (6), 4569-4577.

23. Shannon, R., Revised effective ionic radii and systematic studies of interatomic distances in halides and chalcogenides. Acta Crystallographica Section A 1976, 32 (5), 751-767.

24. Hemp, S. T.; Allen, M. H.; Smith, A. E.; Long, T. E., Synthesis and Properties of Sulfonium Polyelectrolytes for Biological Applications. ACS Macro Letters 2013, 2 (8), 731-735.

25. Mackenzie, M. C.; Shrivats, A. R.; Konkolewicz, D.; Averick, S. E.; McDermott, M. C.; Hollinger, J. O.; Matyjaszewski, K., Synthesis of poly (meth) acrylates with thioether and tertiary sulfonium groups by ARGET ATRP and their use as siRNA delivery agents. Biomacromolecules 2015, 16 (1), 236-245.

26. Su, Y.; Hickey, S. F.; Keyser, S. G. L.; Hammond, M. C., In Vitro and In Vivo Enzyme Activity Screening via RNA-Based Fluorescent Biosensors for S-Adenosyl-Ihomocysteine (SAH). Journal of the American Chemical Society 2016, 138 (22), 7040-7047. 
27. Zhu, D.; Yan, H.; Liu, X.; Xiang, J.; Zhou, Z.; Tang, J.; Liu, X.; Shen, Y., Intracellularly disintegratable polysulfoniums for efficient gene delivery. Advanced Functional Materials 2017, 27 (16), 1606826.

28. Zhao, N.; Lin, X.; Zhang, Q.; Ji, Z.; Xu, F. J., Redox-triggered gatekeeper-enveloped starlike hollow silica nanoparticles for intelligent delivery systems. Small 2015, 11 (48), 6467-6479.

29. Xu, F.; Zhu, Y.; Chai, M.; Liu, F., Comparison of ethanolamine/ethylenediaminefunctionalized poly (glycidyl methacrylate) for efficient gene delivery. Acta biomaterialia 2011, 7 (8), 3131-3140.

30. Xu, F.; Chai, M.; Li, W.; Ping, Y.; Tang, G.; Yang, W.; Ma, J.; Liu, F., Well-defined poly (2-hydroxyl-3-(2-hydroxyethylamino) propyl methacrylate) vectors with low toxicity and high gene transfection efficiency. Biomacromolecules 2010, 11 (6), 1437-1442.

31. Dou, X.; Chai, M.; Zhu, Y.; Yang, W.; Xu, F., Aminated poly (glycidyl methacrylate) $s$ for constructing efficient gene carriers. ACS applied materials \& interfaces 2013, $5(8), 3212-3218$.

32. Venturoli, D.; Rippe, B., Ficoll and dextran vs. globular proteins as probes for testing glomerular permselectivity: effects of molecular size, shape, charge, and deformability. American Journal of Physiology-Renal Physiology 2005, 288 (4), F605-F613.

33. Kulkarni, S. A.; Feng, S.-S., Effects of particle size and surface modification on cellular uptake and biodistribution of polymeric nanoparticles for drug delivery. Pharmaceutical research 2013, 30 (10), 2512-2522.

34. Hoshyar, N.; Gray, S.; Han, H.; Bao, G., The effect of nanoparticle size on in vivo pharmacokinetics and cellular interaction. Nanomedicine 2016, 11 (6), 673-692.

35. Fröhlich, E., The role of surface charge in cellular uptake and cytotoxicity of medical nanoparticles. International journal of nanomedicine 2012, 7, 5577. 
36. Ferguson, C. J.; Hughes, R. J.; Nguyen, D.; Pham, B. T.; Gilbert, R. G.; Serelis, A. K.; Such, C. H.; Hawkett, B. S., Ab initio emulsion polymerization by RAFT-controlled self-assembly. Macromolecules 2005, 38 (6), 2191-2204. 
Chapter 5

186 | $P$ a g e 


\section{Chapter 6}

\section{Transfection efficiency of sulfonium nanogels}

Current efforts in combating diseases caused by genetic defects focus on resolving the source of the disease as opposed to its symptoms. The effects of gene deletions and mutations are ameliorated through gene therapy by means of transfection vectors. These delivery vehicles are tasked with protecting the gene to be incorporated, while transporting it to the cell nucleus. In this chapter we explore cationic polymeric nonviral nucleic acid delivery by nanogels bearing a positive charge on a sulfonium moiety. To our knowledge this is the first attempt at gene transfection by means of sulfonium nanogels. This nanogel (40-THG) was complexed with plasmid DNA pCMV-GFP and its transfection efficiency was trialed at different DNA concentrations. Experiments conducted at various ratios between phosphate containing DNA and sulfonium containing nanogel (S/P ratio) as well as on various cell lines demonstrated that 40-THG does not possess effective gene vector capabilities. Further investigation revealed endocytosis of polyplexes as the obstacle.
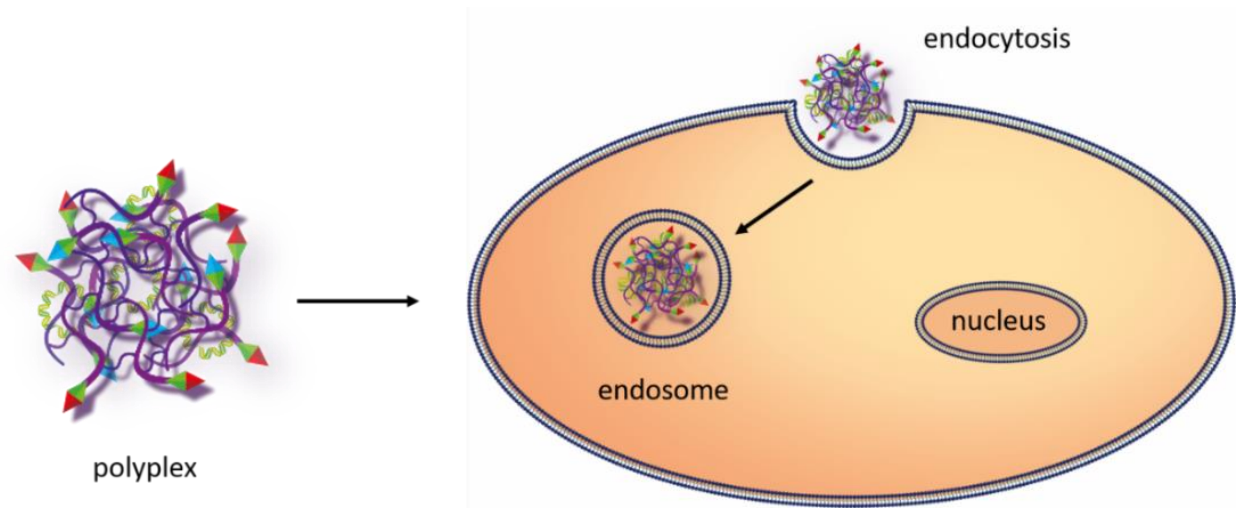


\subsection{Introduction}

Various ailments caused by genetic defects such as type 2 diabetes and certain cancers can be treated by nucleic acid therapy. ${ }^{1-9}$ Likewise, post-transcriptional gene silencing has shown great potential in combating diseases. ${ }^{10}$ Small interfering ribonucleic acids (siRNA) are introduced to the cell nucleus where it interferes with the expression of genes responsible for diseases by degrading the mRNA formed after transcription. In this way gene therapy targets the cause of diseases rather than their symptoms. The therapeutic nucleic acids, however, are met with various challenges, such as enzymatic degradation and repulsion from the equally anionic cell membrane. ${ }^{11-12}$ The use of cationic, nonviral, macromolecule gene carriers such as lipids in the case of the SARS-CoV-2 mRNA vaccine ${ }^{13}$ - greatly resolves these issues. ${ }^{7,14-17}$ The cationic polymer and negatively charged nucleic acid complex to form so-called polyplexes. These polyplexes stimulate cellular uptake through endocytosis. ${ }^{18}$

Since its inception gene transfection has been mostly carried out by means of delivery vehicles containing a nitrogen-based cationic moiety to effectively bind anionic nucleic acids. The most common nitrogen-containing macromolecule used for this purpose is polyethyleneimine (PEI). ${ }^{19}$ Interestingly, the sulfonium cation has a stronger binding affinity for nucleic acids as it has a charge of $+0.67 \mathrm{e}$, meaning it can bind and fully condense DNA at a ratio of approximately $1 .^{20}$ The group of Long at Virginia Tech were the first to utilize the sulfonium functionality in polymeric macrostructures to complex nucleic acids after their initial success with phosphonium macromolecules. ${ }^{21-22}$ Long and coworkers demonstrated successful luciferase expression in HeLa cells utilizing a sulfonium homopolymer and a sulfonium diblock copolymer as delivery vehicles. Others synthesized sulfonium cell penetrating peptides that have low cytotoxicity. ${ }^{23}$ Matyjaszewski et al. studied sulfonium block copolymers as siRNA delivery vehicles in murine clavarial preosteoblasts (MC3T3s) and achieved successful Gapdh knockdown. ${ }^{24}$ 
Investigation of the sulfonium stability in the presence of halides indicated that $<2 \%$ dealkylation occurs in $\mathrm{H}_{2} \mathrm{O}$ after $48 \mathrm{~h}$ at a 5 -fold molar excess of halide to $\mathrm{S}^{+}$. The half-life of the sulfonium species in $\mathrm{D}_{2} \mathrm{O}$ under biological conditions (120 mM halide ion) was determined to be more than 100 days. Shen and coworkers further progressed sulfonium gene carriers by synthesizing polysulfoniums that fragment to neutral components, once reduced by the elevated reactive oxygen species present in tumor cells. ${ }^{25-26}$ In this way therapeutic cargo is quickly released into the cytosol - achieving higher transfection efficiency than the nondegradable polysulfonium equivalent.

Diversifying the supply of cationic gene carriers provides versatile and biocompatible alternatives to nitrogen-based gene delivery platforms. Although still in its infancy, this field of sulfonium polymeric particles as vectors shows great promise. In this chapter we investigate the transfection properties of the polyplexes described in Chapter 5.

\subsection{Results and Discussion}

\subsubsection{In vitro transfection efficiency and cytotoxicity.}

To assess the transfection efficiency of sulfonium nanogel 40-THG, experiments were conducted in human cervical cancer cells (HeLa) with plasmid DNA encoding for green fluorescent protein (GFP). Branched polyethyleneimine at $25 \mathrm{kDa}\left(\mathrm{bPEI}_{25 \mathrm{k}}\right)$ was used as a positive control along with commercial lipid vector Lipofectamine ${ }^{\mathrm{TM}} 3000$. Various ratios between 40-THG and plasmid DNA were evaluated in a 96-well plate under serum-free (in $\mathrm{DMEM}^{-}$) conditions. Transfection experiments were carried out by incubating HeLa cells (seeded at 15.000 cells per well) with plasmid DNA encoding for green fluorescent protein (pCMV-GFP, $0.25 \mu \mathrm{g}$ per well) for $6 \mathrm{~h}$ in DMEM followed by an additional $40 \mathrm{~h}$ in 
Chapter 6

$\mathrm{DMEM}^{+}$to allow GFP expression. The transfection efficiency was qualitatively analyzed by fluorescence microscopy as seen in Figure 1. The cell viability was quantitatively analyzed with a plate reader and compared to cells that were not exposed to the transfection medium (Figure S1 in supplementary data). Transfection and cytotoxicity experiments were conducted in parallel meaning microscopy photos and metabolic activity were measured at the same time point. GFP expression was only observed in HeLa cells at an S/P ratio of 100. 

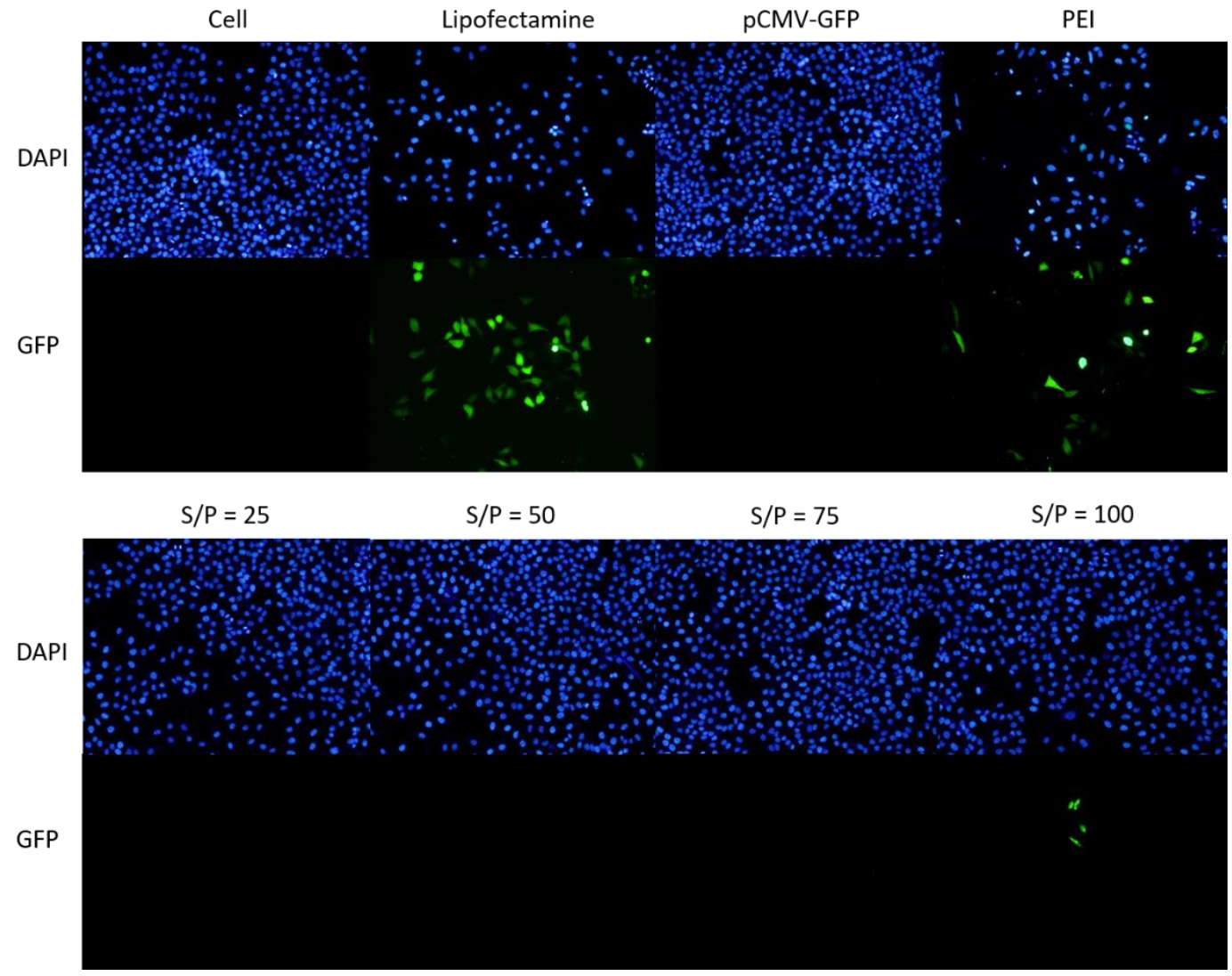

Figure 1. Fluorescence microscope images of HeLa cells transfected by pCMV-GFP via 40-THG taken at $\lambda=420$ $\mathrm{nm}$ to image DAPI stained cell membranes and at $\lambda=525 \mathrm{~nm}$ to image GFP. The top row depicts the controls: HeLa cells that were not exposed to transfection media, Lipofectamine ${ }^{\mathrm{TM}} 3000$ as positive control prepared according to instructions provided by manufacturer, pCMV-GFP signifying the plasmid DNA without any vectors and $\mathrm{bPEI}_{25 \mathrm{k}}$ at $\mathrm{N} / \mathrm{P}=25$ also serving as positive control. 100x magnification. (S/P signifies the amount of sulfonium moieties in the nanogel divided by the amount of phosphate moieties in the plasmid DNA. Likewise $\mathrm{N} / \mathrm{P}$ denotes the number of nitrogen atoms in $\mathrm{bPEl}_{25 \mathrm{k}}$ divided by the number of phosphor atoms in the plasmid DNA.)

As seen in Figure 1, minimal to no GFP expression was observed for $\mathbf{4 0 - T H G . ~ F i g u r e ~} 1$ also illustrates the high toxicity of Lipofectamine ${ }^{\mathrm{TM}} 3000$ and $\mathrm{bPEI}_{25 \mathrm{k}}$ in comparison to the 40- 
THG polyplexes. A high positive charge is toxic to cells resulting in $\mathrm{bPEl}_{25 \mathrm{k}}$ and its polyplexes being intrinsically toxic. ${ }^{27}$ This was further confirmed during the cytotoxicity studies as depicted in Figure S1, resulting in low cell viability. The 40-THG polyplexes contrarily are non-toxic, displaying cell viability similar to cells that were not exposed to the polyplexes.

To allow higher uptake and transfection efficiency the experiment was repeated with a longer incubation period of 20h (Figure 2 and 3 ) as well as at a higher DNA/well concentration of $1 \mu \mathrm{g}$ (Figure 3). Transfection and cytotoxicity experiments were carried out in parallel meaning microscopy photos and metabolic activity were measured at the same time point. Once the polyplexes were removed the cells were incubated for an additional $24 \mathrm{~h}$ to allow GFP expression. Cells were seeded at 15.000 cells per well. 

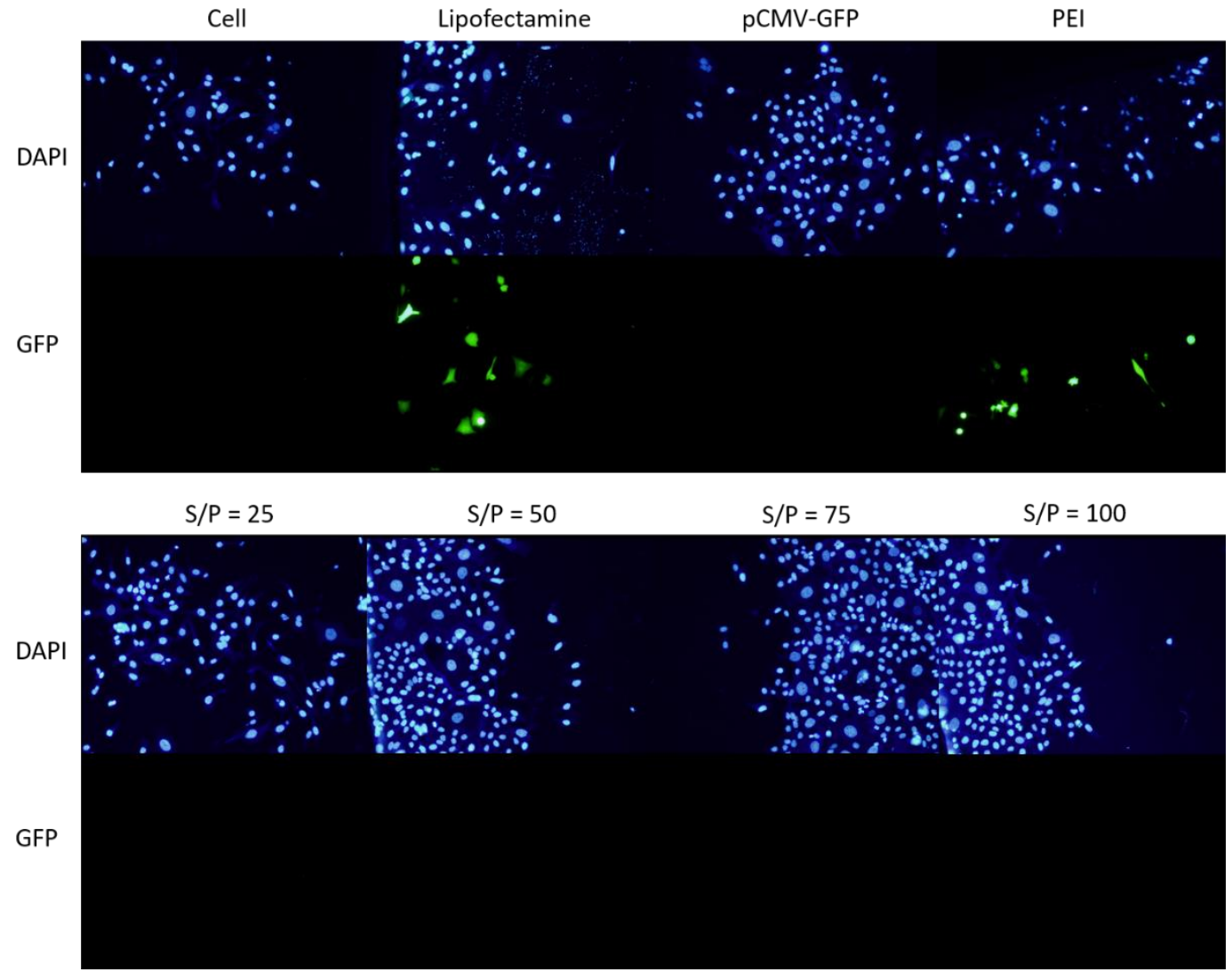

Figure 2. Fluorescence microscope images of HeLa cells treated for $20 \mathrm{~h}$ by 40 -THG polyplexes carrying plasmid DNA encoding for GFP (pCMV-GFP, $0.25 \mu \mathrm{g}$ per well). 100x magnification. 


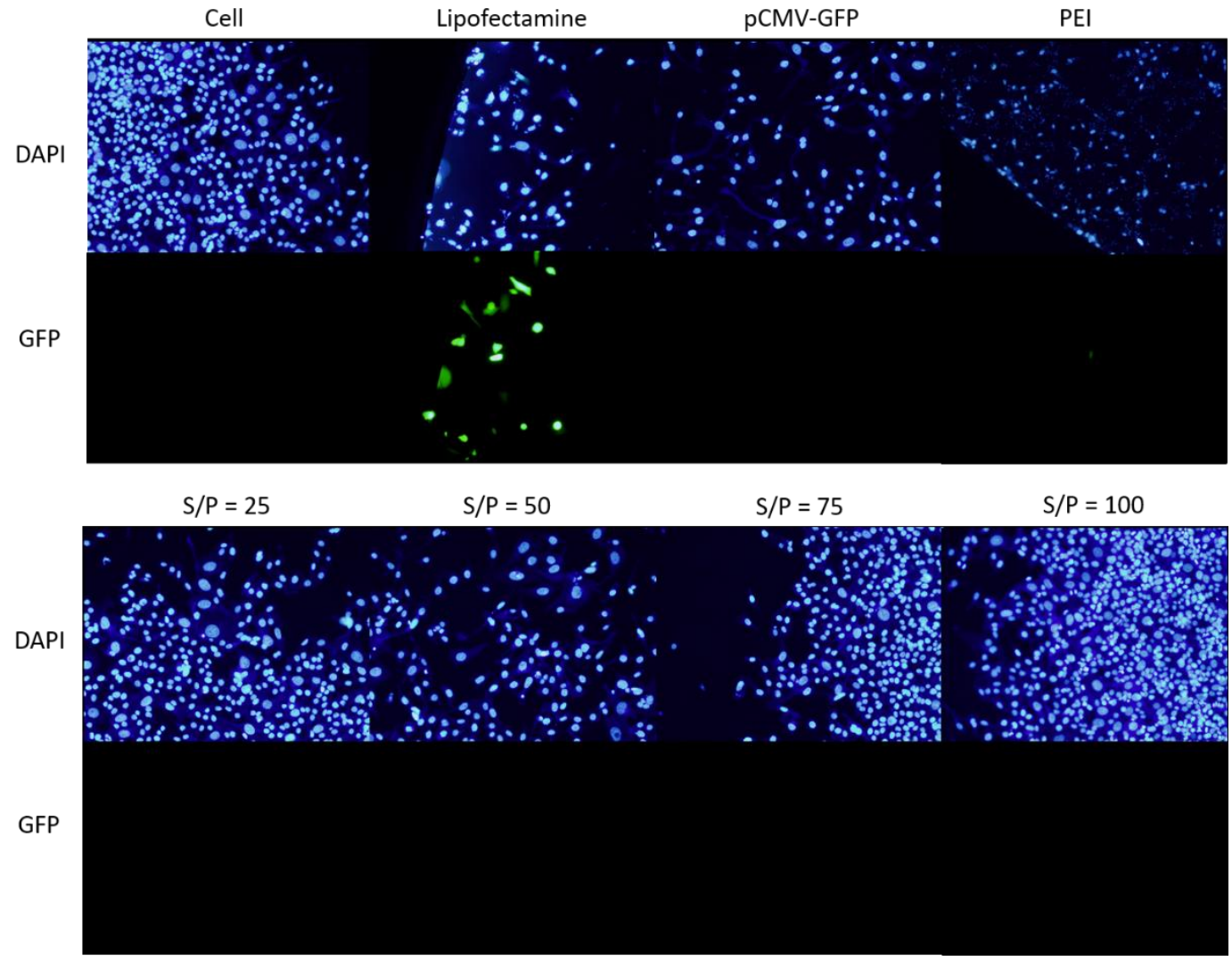

Figure 3. Fluorescence microscope images of HeLa cells transfected for $20 \mathrm{~h}$ by pCMV-GFP ( $1 \mu \mathrm{g}$ per well) via 40-THG. 100x magnification.

Both Figure 2 and 3 exhibit no GFP expression for the 40-THG polyplexes. A longer incubation period did not increase transfection efficiency. The incubation time also had a negative impact on the HeLa cells as significant reduction in living cells is observed. This is most likely due to the fact that serum-free medium (DMEM $\left.{ }^{-}\right)$is used during this incubation period. The cells do not tolerate the absence of FBS for such an extended period of time. An increased incubation period with the 40-THG polyplexes and a higher plasmid DNA concentration had no effect on the cell viability of HeLa cells. On the contrary, longer 
incubation with $\mathrm{bPEl}_{25 \mathrm{k}}$ polyplexes seems to significantly impact the viability of the HeLa cells (Figure S2).

It is known that the transfection efficiency varies between different cell lines for the same vector. ${ }^{28}$ This feature is attractive as it can be exploited to achieve selectivity and enhance targeting. Bearing this in mind various cell lines were subjected to transfection experiments to evaluate the transfection efficiency and potential selectivity of 40-THG polyplexes. HeLa (human cervical cancer), bEnd.3 (mouse endothelial brain), HepG2 (human epithelial liver cancer), C2C12 (mouse muscle myoblasts), RAW264 (mouse macrophages), HEK293 (human embryonic kidney cells) and HFF (human fibroblast) cells were incubated with polyplexes for $5 \mathrm{~h}$ in serum-free medium (DMEM-) followed by 44h of incubation to allow GFP expression. All experiments were carried out at $1 \mu \mathrm{g}$ pCMV-GFP and 15.000 cells per well in a 96-well plate. The number of polyplex ratios was increased from four to five and the S/P ratio was increased to include 125 and 150. Microscopy photos and metabolic activity are from the same time point. 


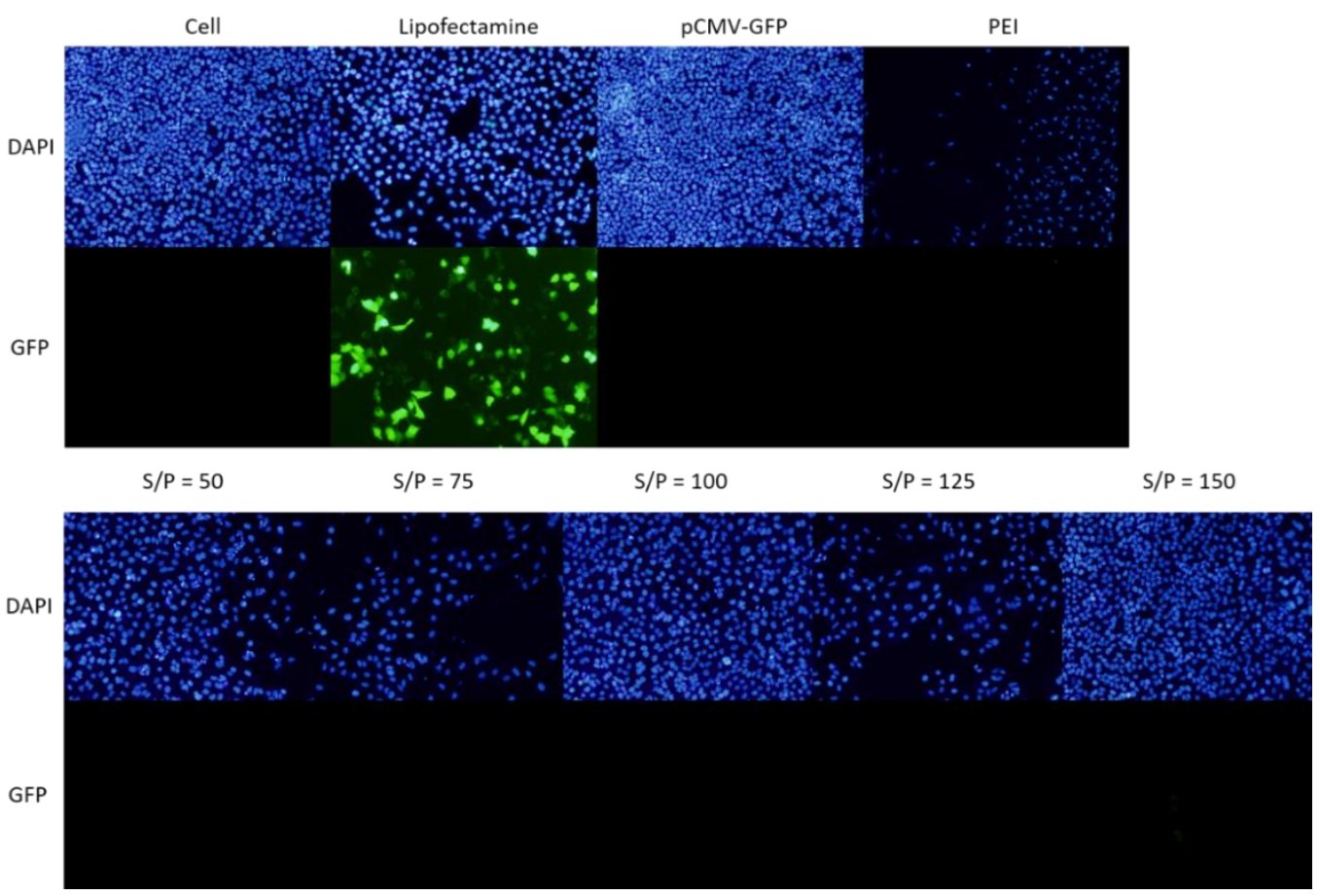

Figure 4. HeLa cells were imaged under a fluorescent microscope after transfection experiments with polyplexes carrying pCMV-GFP. The cells were incubated for $5 \mathrm{~h}$ to allow uptake of the polyplexes followed by incubation for $44 \mathrm{~h}$ to allow GFP expression. No expression was observed in the HeLa cells exposed to 40-THG polyplexes. 


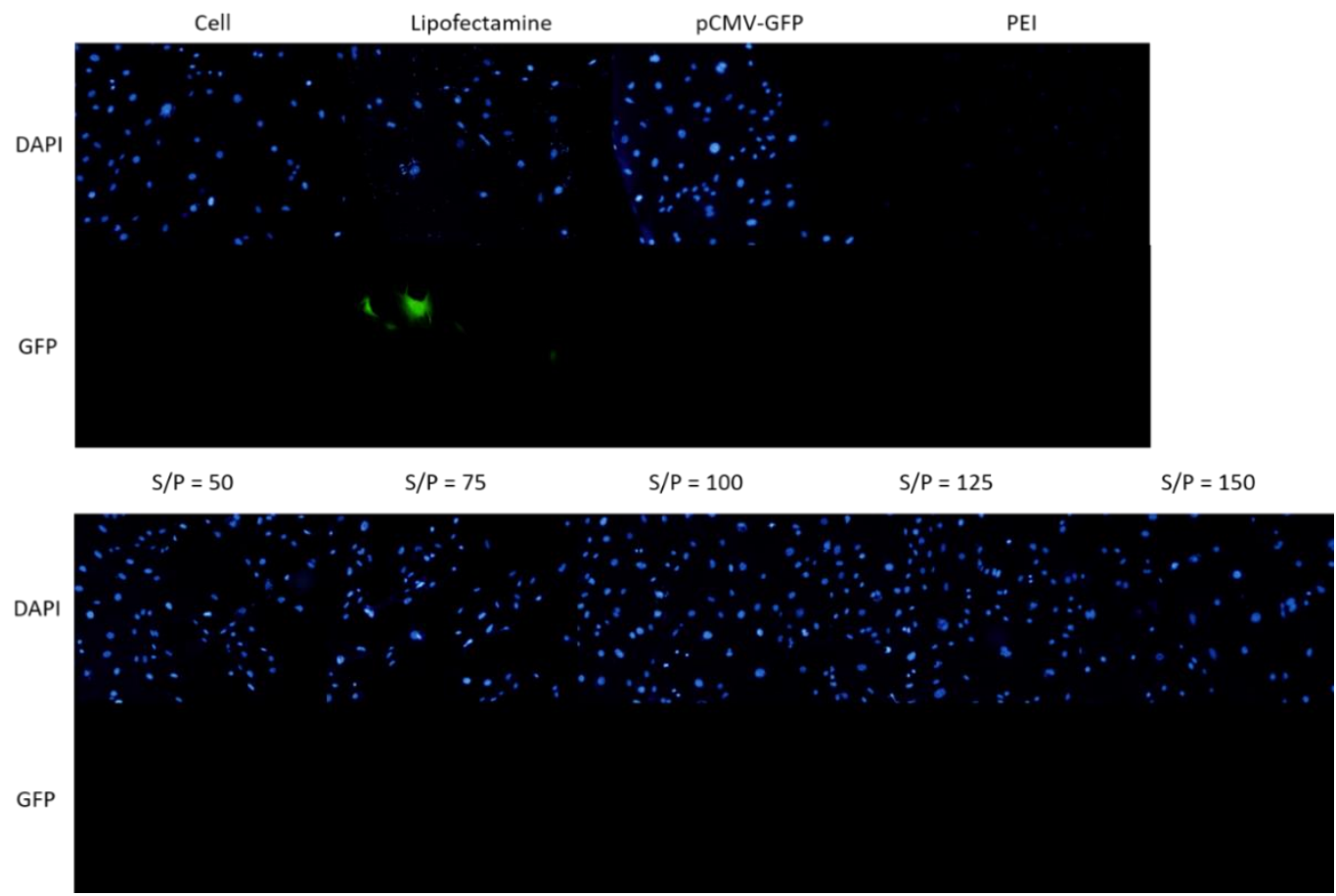

Figure 5. bEnd.3 cells were imaged under a fluorescent microscope after transfection experiments with polyplexes carrying pCMV-GFP. The cells were incubated for $5 \mathrm{~h}$ to allow uptake of the polyplexes followed by incubation for $44 \mathrm{~h}$ to allow GFP expression. No expression was observed in the bEnd.3 cells exposed to 40 THG polyplexes. 


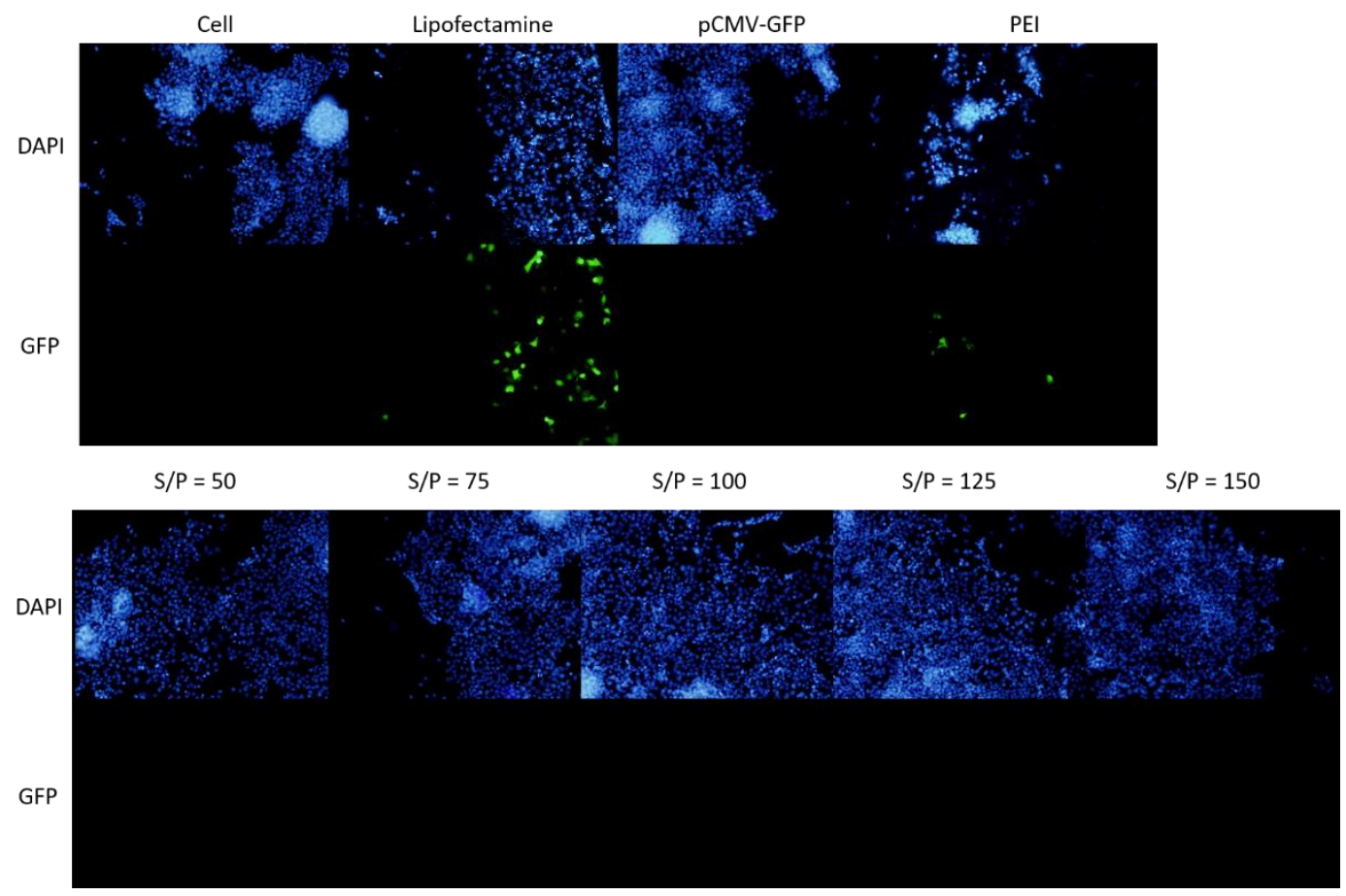

Figure 6. HepG2 cells were imaged under a fluorescent microscope after transfection experiments with polyplexes carrying pCMV-GFP. The cells were incubated for $5 \mathrm{~h}$ to allow uptake of the polyplexes followed by incubation for $44 \mathrm{~h}$ to allow GFP expression. No expression was observed in the HepG2 cells exposed to 40THG polyplexes. 


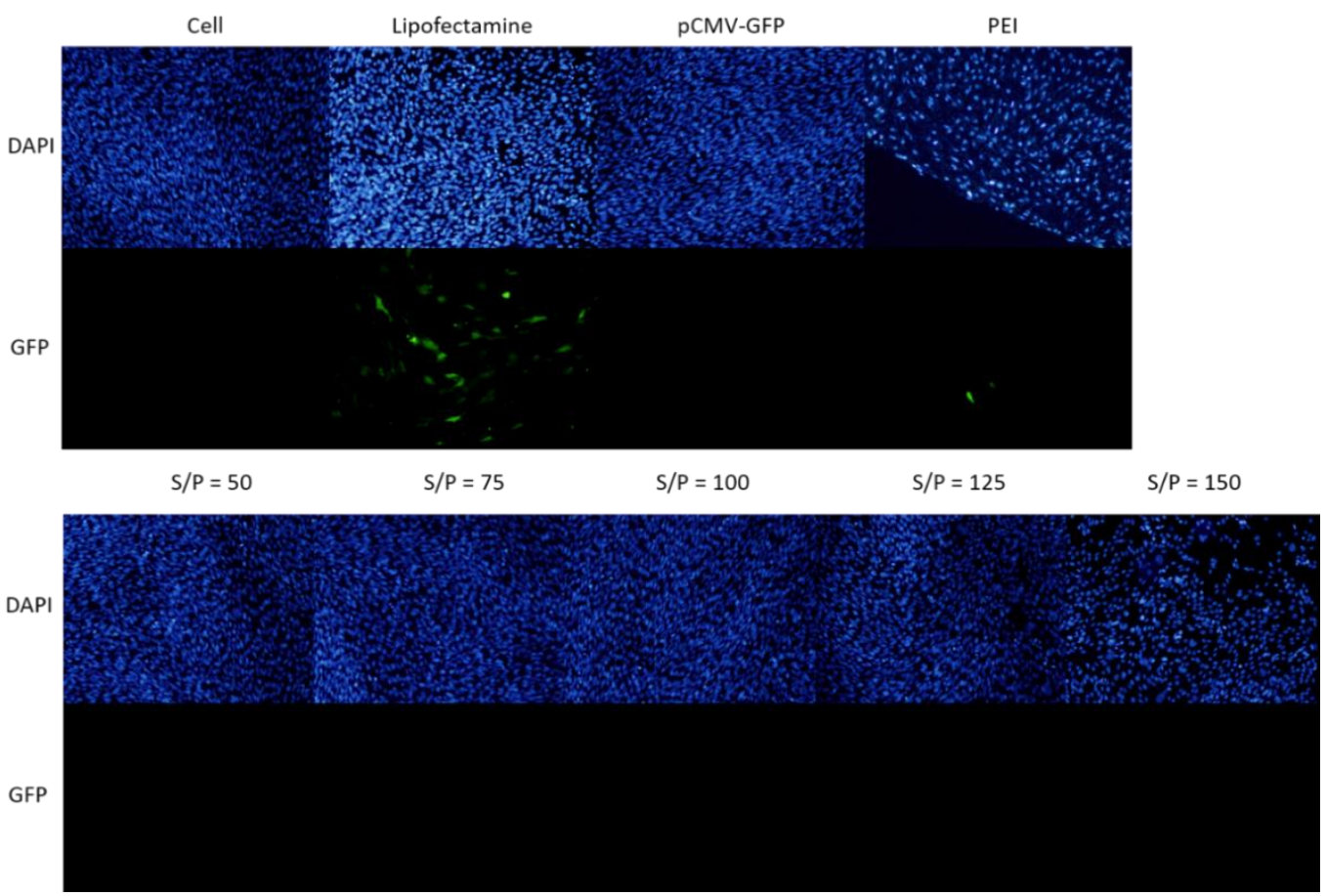

Figure 7. $\mathrm{C} 2 \mathrm{C} 12$ cells were imaged under a fluorescent microscope after transfection experiments with polyplexes carrying pCMV-GFP. The cells were incubated for $5 \mathrm{~h}$ to allow uptake of the polyplexes followed by incubation for $44 \mathrm{~h}$ to allow GFP expression. No expression was observed in the C2C12 cells exposed to 40 THG polyplexes. 

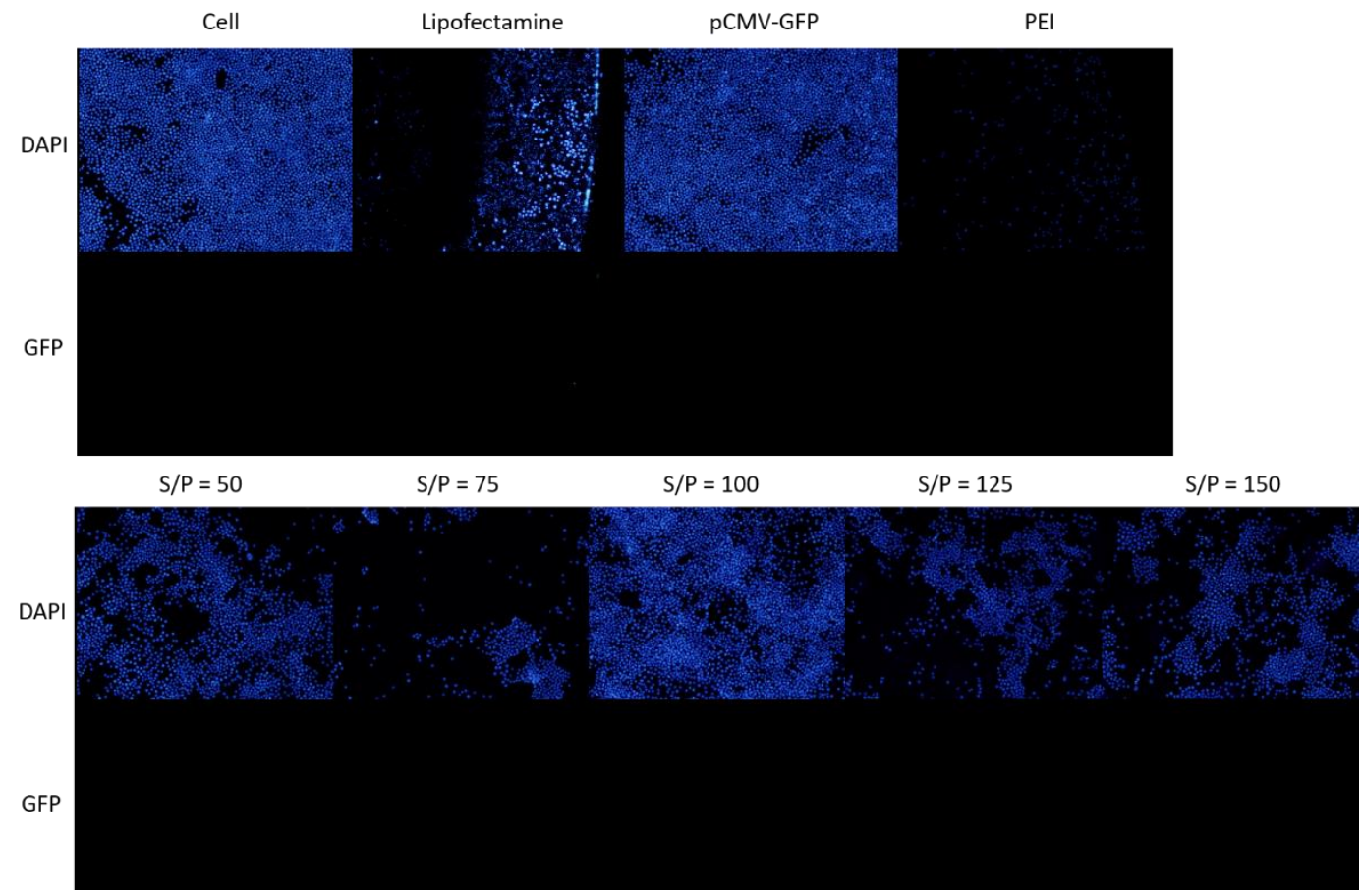

Figure 8. RAW264 cells were imaged under a fluorescent microscope after transfection experiments with polyplexes carrying pCMV-GFP. The cells were incubated for $5 \mathrm{~h}$ to allow uptake of the polyplexes followed by incubation for $44 \mathrm{~h}$ to allow GFP expression. No expression was observed in the RAW264 cells exposed to 40THG polyplexes. 


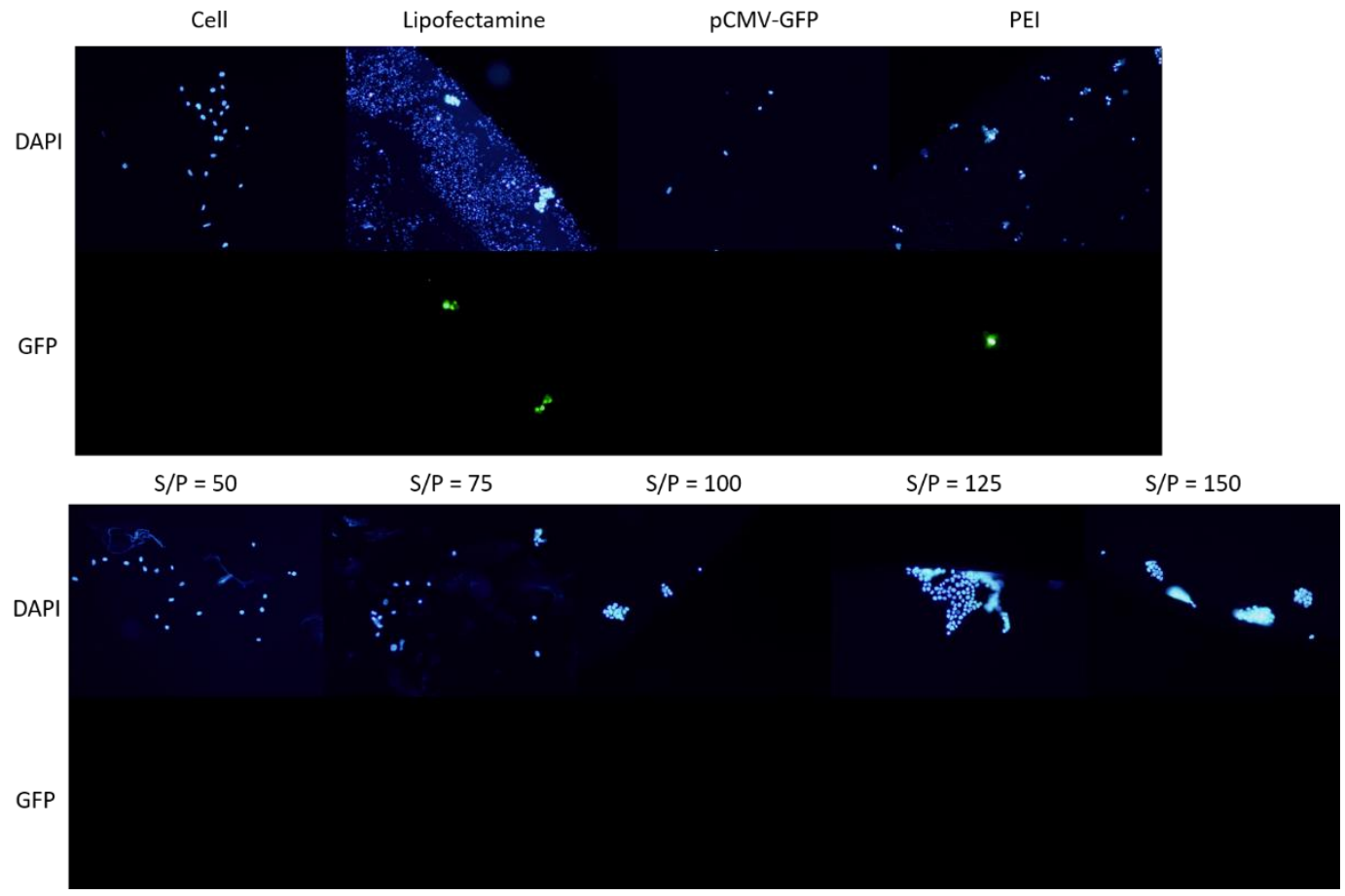

Figure 9. HEK293 cells were imaged under a fluorescent microscope after transfection experiments with polyplexes carrying pCMV-GFP. The cells were incubated for $5 \mathrm{~h}$ to allow uptake of the polyplexes followed by incubation for $44 \mathrm{~h}$ to allow GFP expression. No expression was observed in the HEK293 cells exposed to 40THG polyplexes.

Although, HEK293 cells were also seeded at 15.000 cells/well, still very few cells are seen in Figure 9. This is a result of HEK293 cells being semi-adherent and thus not adequately attaching to the well plate. As HEK293 cells are known to be easily transfected, optimization of their adherence properties is expected..$^{29}$ However, this set of experiments clearly indicate that $\mathbf{4 0 - T H G}$ is not adequate for gene transfection purposes. Optimization of cell adherence would most likely be time consuming and result in minor, if any, GFP expression. 


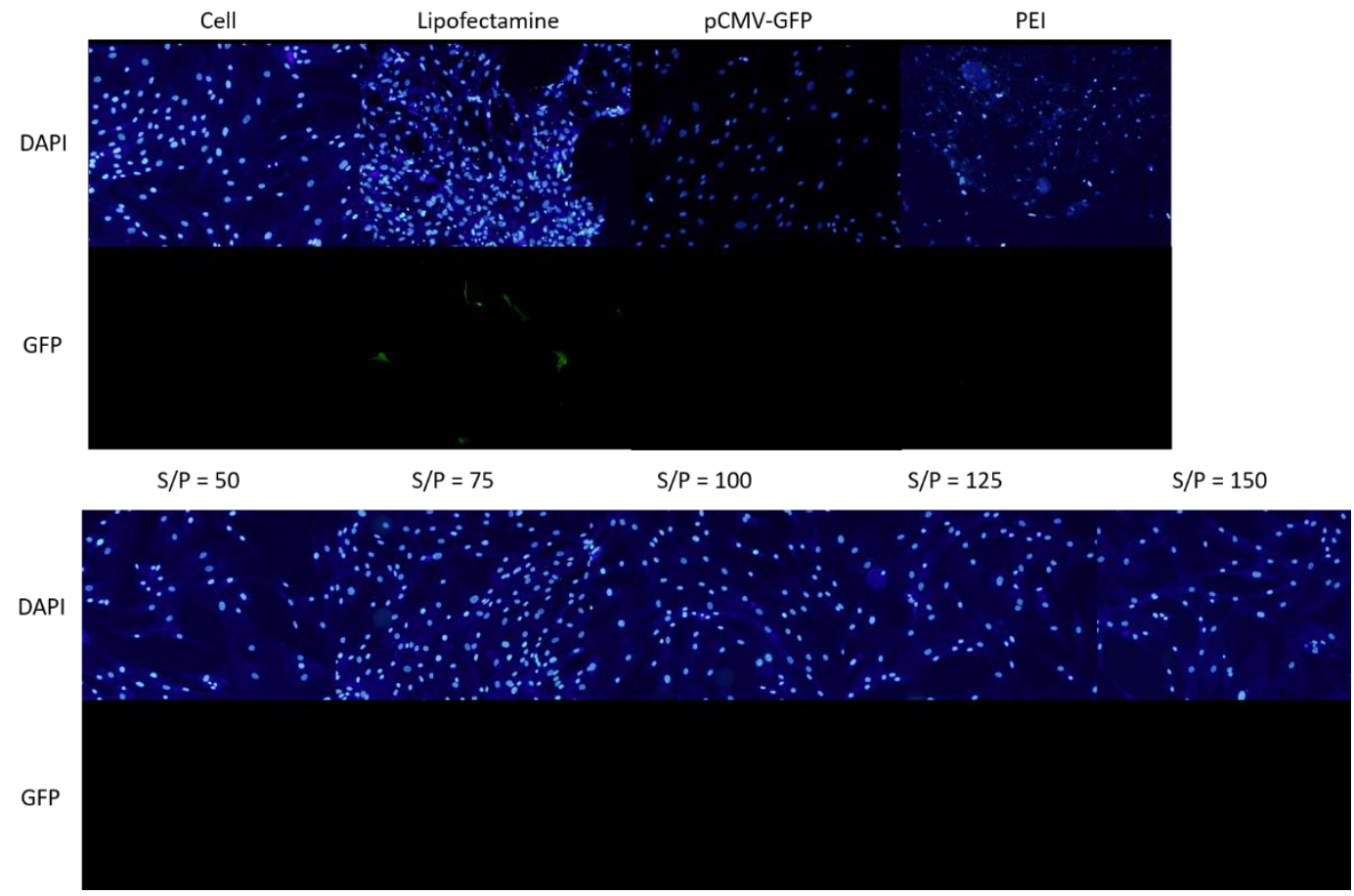

Figure 10. HFF cells were imaged under a fluorescent microscope after transfection experiments with polyplexes carrying pCMV-GFP. The cells were incubated for $5 \mathrm{~h}$ to allow uptake of the polyplexes followed by incubation for $44 \mathrm{~h}$ to allow GFP expression. No expression was observed in the HFF cells exposed to 40-THG polyplexes.

The polyplexes did not exhibit GFP expression or any significant cytotoxicity for the cell lines mentioned above. Lipofectamine ${ }^{\mathrm{TM}} 3000$ and especially bPEI $_{25 \mathrm{k}}$ showed high levels of cytotoxicity, resulting in a minimum of $40 \%$ cell death (data not shown). Figures $4-10$ indicate reduced tolerance for a concentration of $1 \mu \mathrm{g}$ pCMV-GFP per well compared to $0.25 \mu \mathrm{g}$ pCMV-GFP per well (Figure 1 and 2). Notably, cells transfected by $\mathrm{bPEl}_{25 \mathrm{k}}$ polyplexes exhibited significant cell death and reduced transfection. As such all following experiments were done at $0.25 \mu \mathrm{g}$ pCMV-GFP per well. 
Having tested 7 different cell lines and observing no transfection, the conclusion can be drawn that 40-THG does not function as a vector for gene transfection. The schematic representation of gene transfection in Figure 11 indicates that successful transfection resulting in GFP gene expression requires a series of subsequent events all occurring successfully.

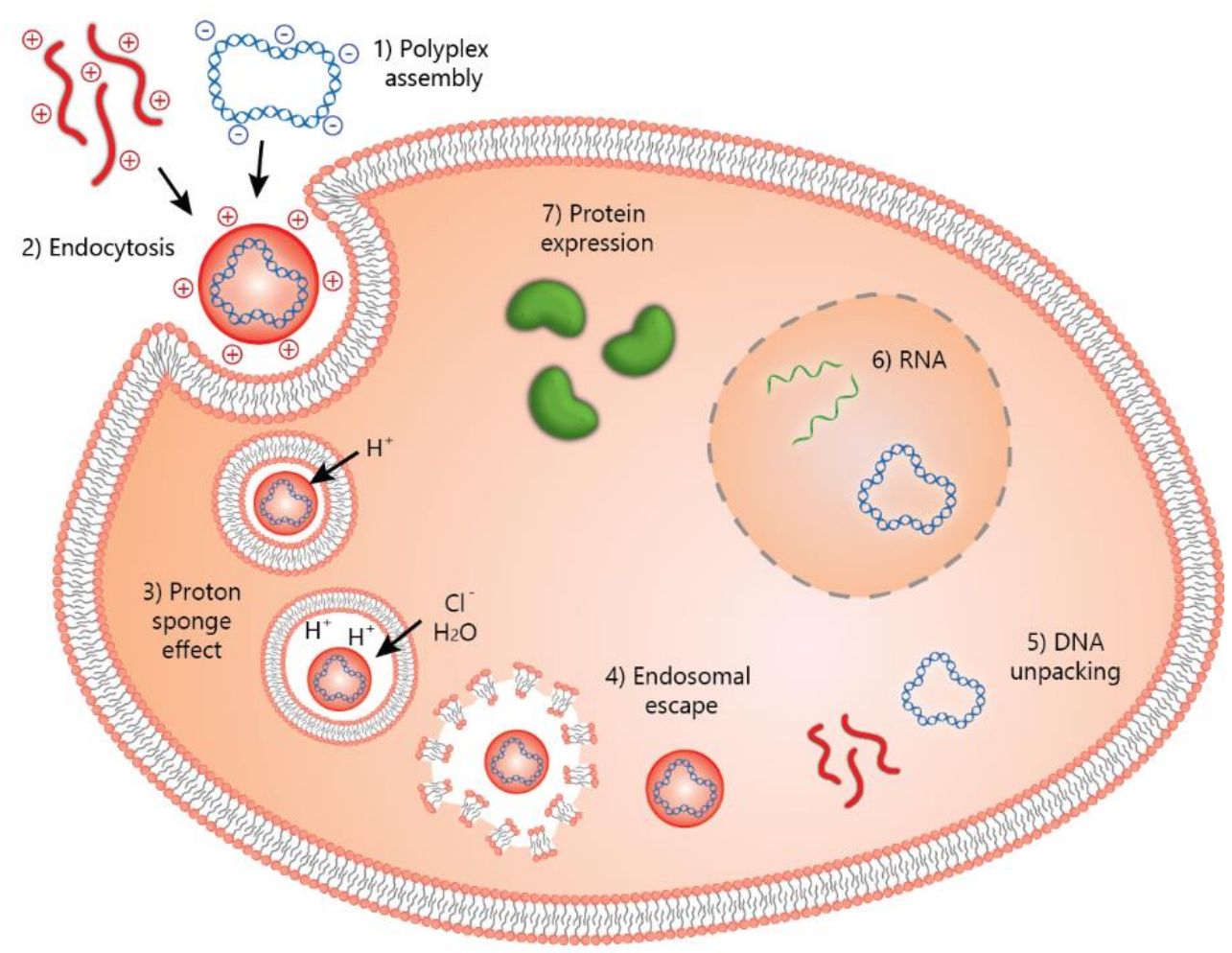

Figure 11. A schematic representation of gene transfection. Negatively charged plasmid DNA interacts with a positively charged gene vector forming a positively charged polyplex. This polyplex interacts through multivalent interactions with the negatively charged cell membrane resulting in endocytosis of the polyplex, followed by endosomal escape through the proton sponge effect. Once inside the cytosol the polyplex will collapse into the vector and plasmid DNA which can then make its way to the cell nucleus. Here, the DNA is transcribed resulting in expression of a protein, such as GFP when pCMV-GFP is used. Reprint from ref ${ }^{30}$ with permission. 
The experiments carried out in Chapter 5 indicate that step 1 of gene transfection as depicted in Figure 11 was successful as these polyplexes can be imaged (TEM) and their properties can be measured (DLS, zeta potential). Protein expression (step 7) was also successful as seen for Lipofectamine ${ }^{\mathrm{TM}} 3000$ and $\mathrm{bPEI}_{25 \mathrm{k}}$ in the figures above. The reason for the unsuccessful transfection experiments thus lies with the remaining 5 steps. As stated previously the ideal gene vector has a positive charge that interacts with the negative charge of DNA forming a polyplex. The ideal gene vector enhances uptake of the plasmid $\mathrm{DNA}^{31-32}$, protects it from interacting with proteins in the cytosol or from degradation by intracellular nucleases ${ }^{33-34}$ and transports it to the cell nucleus and across the nucleus membrane ${ }^{35}$. As such the vector is expected to barricade the plasmid in some form, for example through encapsulation. Considering that the polyplexes are formed by mixing spherical nanogels with relatively large plasmid DNA, it is expected that the DNA does not enter the nanogel matrix but rather sits on top of it, interacting with the positive charges on its surface. As a result, the plasmid DNA is not adequately protected. Most likely the plasmid DNA blankets a grand majority, if not all, of the positive surface charges. Having few remaining available positive moieties left on its surface, uptake of the nanogel is significantly hindered as it is no longer capable of properly interacting with the negatively charged cell membrane. Another possible cause is the stability of the sulfonium moiety and the formed polyplexes. ${ }^{36}$ DLS measurements (Chapter 5 ) indicate that the polyplexes are not stable and start disassembling within $3 \mathrm{~h}$. Both of these factors would lead to minimal uptake, thus failure at step 2.

In order to test these hypotheses transfection experiments were conducted with DTAF-labelled 40-THG sulfonium nanogel, in order to visualize the nanogels and monitor their uptake. Polyplexes were formed with PCMV-lacZ and DTAF-labeled 40-THG to evaluate the influence of bound DNA on polyplex uptake without the interference of the green fluorescence of GFP. 40-THG was also incubated with GFP protein as it is 
significantly smaller than PCMV-GFP and thus more likely to enter the nanogel matrix. Lastly, linear sulfonium polymers (synthesized from pGMA), equivalent to 40-THG, were synthesized to evaluate whether they form more effective polyplexes than their crosslinked nanogel counterparts. The choice was made for HeLa cells, $\mathrm{C} 2 \mathrm{C} 12$ cells and HEK293 cells (at a seed density of 10.000 cells per well) as they showed most promise. The cells were incubated with polyplexes/40-THG/GFP protein for $2 \mathrm{~h}$ in serum-free medium (DMEM-) followed by $48 \mathrm{~h}$ of incubation to allow GFP expression for the polyplexes carrying PCMV-GFP. All experiments were carried out in duplo and at $0.25 \mu \mathrm{g}$ pCMV-GFP per well (or its equivalent) in a 96-well plate. Polymer polyplexes were incubated for $45 \mathrm{~min}$ at RT prior to use and $\mathbf{4 0 - T H G}$ polyplexes for $10 \mathrm{~min}$ at RT prior to use. Microscopy photos and metabolic activity are from the same time point. 

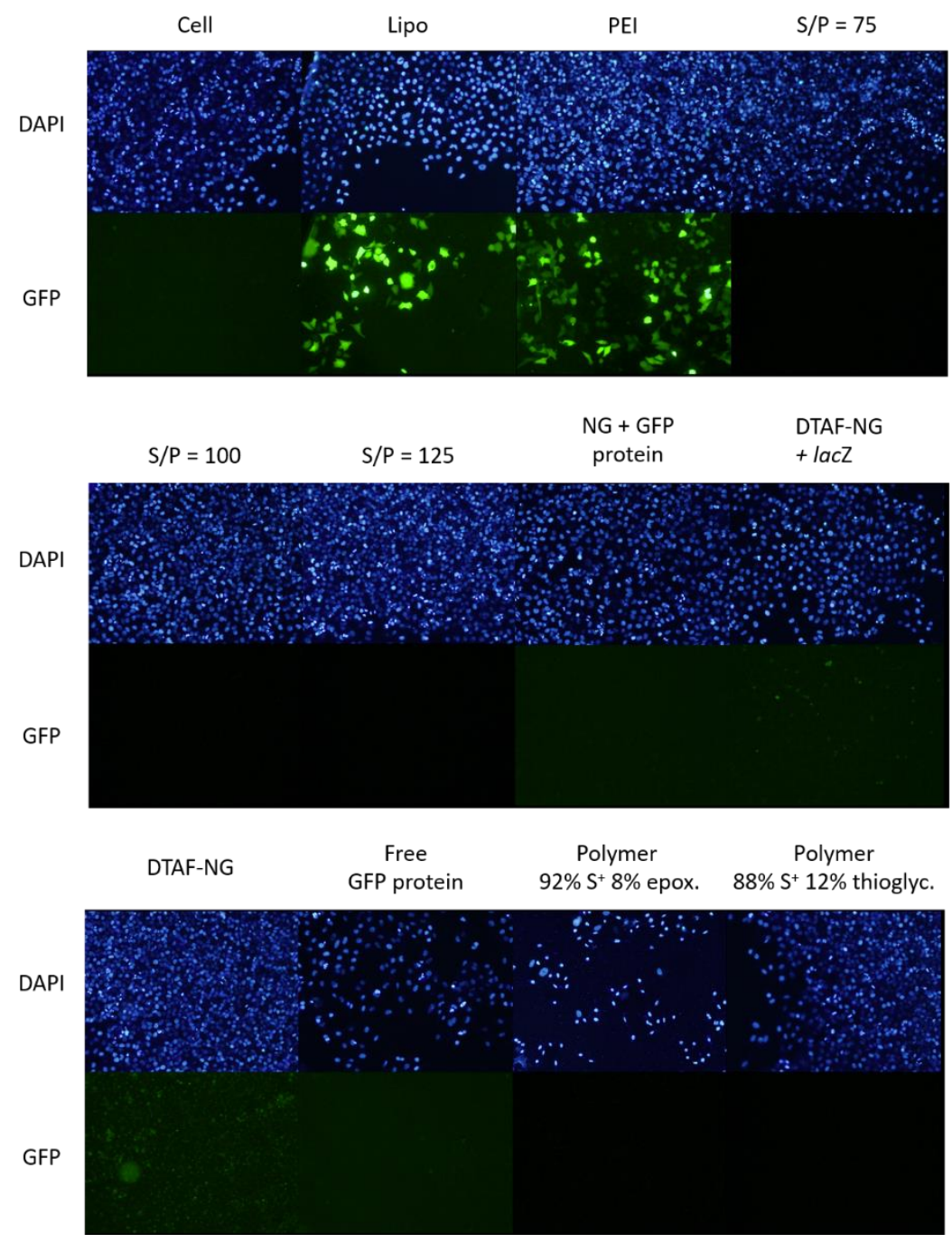

Figure 12. Transfection with 40-THG at $S / P=75,100$ and 125 on HeLa cells was repeated, along with Lipofectamine $^{\mathrm{TM}} 3000$ and bPEI $25 \mathrm{k}$ as control. Uptake was evaluated for GFP protein carried in nanogel (middle row, $3^{\text {rd }}$ column), lacZ polyplexes $\left(S / P=125\right.$, middle row, $4^{\text {th }}$ column $)$ in dyed nanogel, empty dyed nanogels (41 $\mathrm{nm}, \zeta=-11 \mathrm{mV}$, bottom row, $1^{\text {st }}$ column) and free GFP protein (bottom row, $2^{\text {nd }}$ column). Transfection efficiency was monitored for GFP polyplexes formed from sulfonium polymers carrying either an epoxide moiety $\left(S / P=50,259 \mathrm{~nm},+37 \mathrm{mV}\right.$, bottom row, $3^{\text {rd }}$ column) or a thioglycerol moiety $(S / P=94,90 \mathrm{~nm},+16$ $\mathrm{mV}$, bottom row, $4^{\text {th }}$ column). 

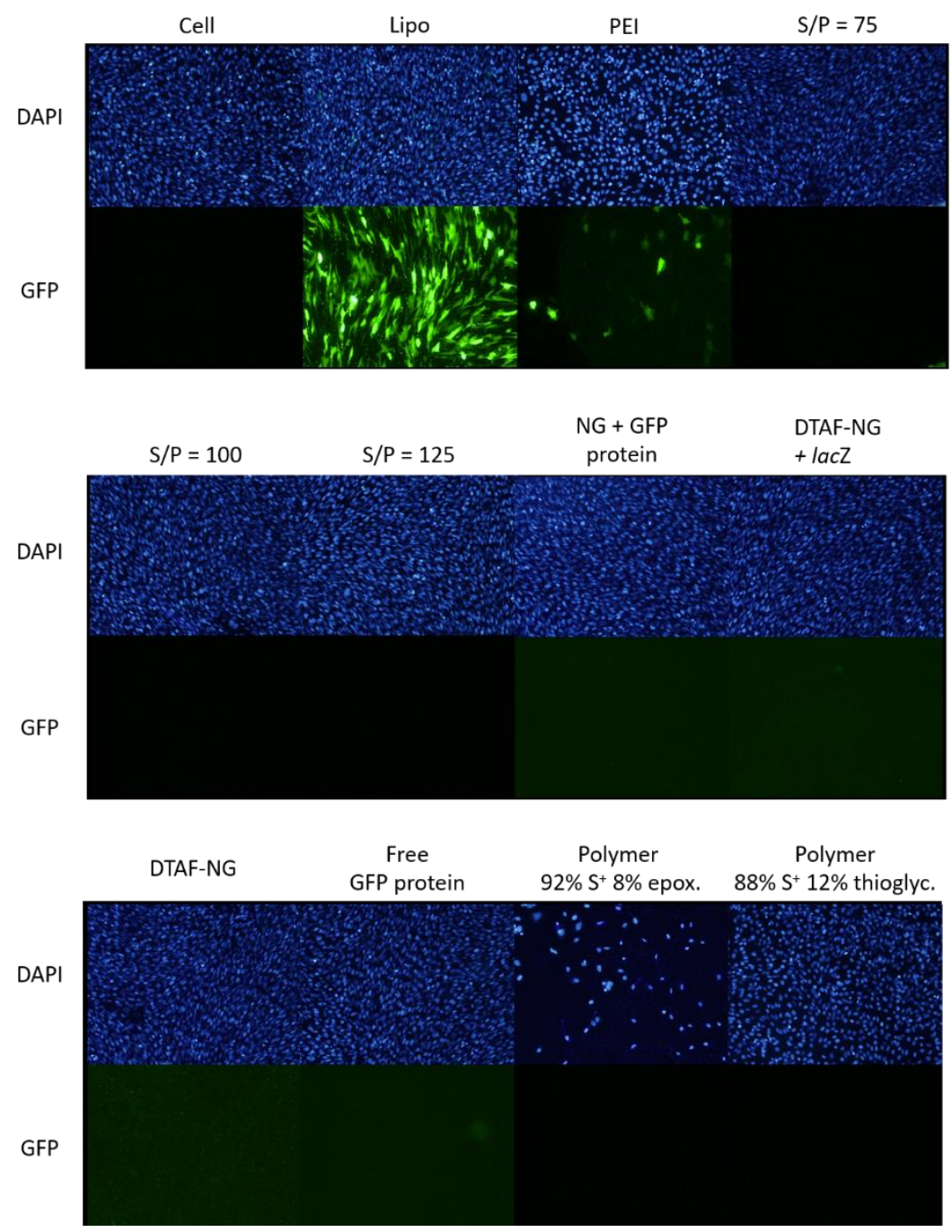

Figure 13. Transfection with 40-THG at $S / P=75,100$ and 125 on $C 2 C 12$ cells was repeated, along with Lipofectamine $^{\mathrm{TM}} 3000$ and $\mathrm{bPEI}_{25 \mathrm{k}}$ as control. Uptake was evaluated for GFP protein carried in nanogel (middle row, $3^{\text {rd }}$ column), lacz polyplexes $\left(S / P=125\right.$, middle row, $4^{\text {th }}$ column) in dyed nanogel, empty dyed nanogels (41 $\mathrm{nm}, \zeta=-11 \mathrm{mV}$, bottom row, $1^{\text {st }}$ column) and free GFP protein (bottom row, $2^{\text {nd }}$ column). Transfection efficiency was monitored for GFP polyplexes formed from sulfonium polymers carrying either an epoxide moiety $\left(S / P=50,259 \mathrm{~nm},+37 \mathrm{mV}\right.$, bottom row, $3^{\text {rd }}$ column) or a thioglycerol moiety $(S / P=94,90 \mathrm{~nm},+16$ $\mathrm{mV}$, bottom row, $4^{\text {th }}$ column). 

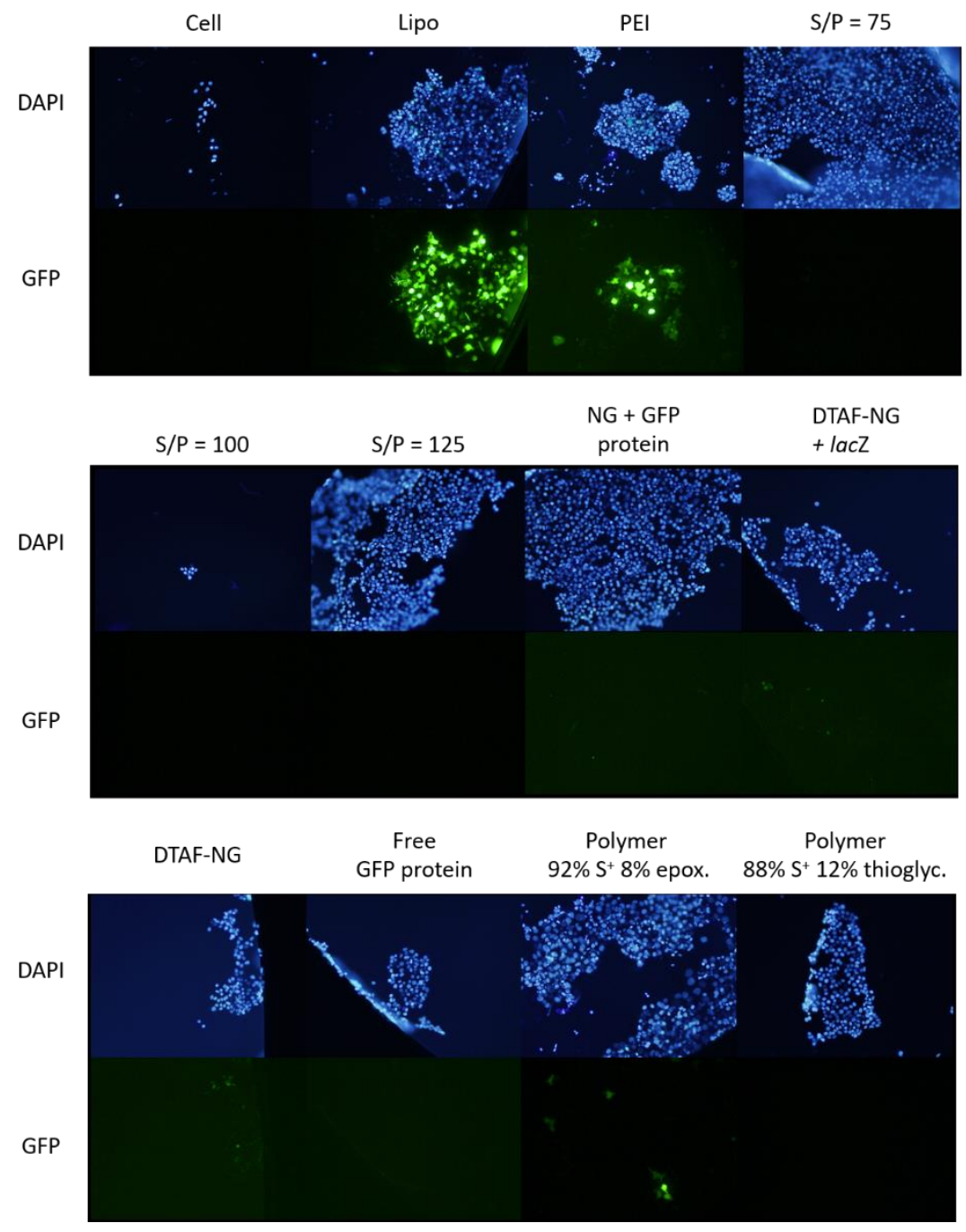

Figure 14. Transfection with 40-THG at $S / P=75,100$ and 125 on HEK293 cells was repeated, along with Lipofectamine $^{\mathrm{TM}} 3000$ and bPEI $25 \mathrm{k}$ as control. Uptake was evaluated for GFP protein carried in nanogel (middle row, $3^{\text {rd }}$ column), lacZ polyplexes $\left(S / P=125\right.$, middle row, $4^{\text {th }}$ column $)$ in dyed nanogel, empty dyed nanogels (41 $\mathrm{nm},-11 \mathrm{mV}$, bottom row, $1^{\text {st }}$ column) and free GFP protein (bottom row, $2^{\text {nd }}$ column). Transfection efficiency was monitored for GFP polyplexes formed from sulfonium polymers carrying either an epoxide moiety $\left(S / P=50,259 \mathrm{~nm},+37 \mathrm{mV}\right.$, bottom row, $3^{\text {rd }}$ column) or a thioglycerol moiety $(S / P=94,90 \mathrm{~nm},+16$ $\mathrm{mV}$, bottom row, $4^{\text {th }}$ column). 
Figures 12 - 14 show no improvement in the transfection efficiency of 40-THG polyplexes conducted at lower plasmid DNA concentration. They do however indicate less cell death and thus a higher tolerance at $0.25 \mu \mathrm{g}$ pCMV-GFP per well. Although slightly better than before, HEK293 cells still exhibited poor adherence to the well plate. Figures 12 and 13 nicely highlight the cytotoxicity of epoxides. ${ }^{37}$ Interestingly, transfection was only witnessed in HEK293 cells for polyplexes formed from linear sulfonium polymers bearing $8 \%$ epoxides. It was hypothesized that linear polymers have higher transfection efficiencies than nanogels as they have more freedom of movement and can thus better encapsulate pDNA. If this were truly the case and linear polymers were indeed far better at transfection, both sulfonium polymers would exhibit much higher GFP expression than what is seen in Figures $12-14$. As such, the experiments conducted in this chapter do not definitively rule out nanogels as gene vectors. As both the sulfonium nanogels as well as the polysulfoniums fail to transfect (properly), the cause most likely does not lie in the shape of the polymeric vector.

Uptake of GFP protein was also unsuccessful - both for free GFP protein and GFP protein mixed with 40-THG. This protein however does not have a negative surface charge facilitating its complexation with 40-THG - meaning it most likely diffuses out of the nanogel matrix. As such the efficiency of 40-THG at transporting proteins across cellular membranes remains unknown. As seen in Figures 12 - 14, uptake is witnessed for free/empty dyed 40-THG (bottom row, $3^{\text {rd }}$ column) and polyplexes formed from dyed 40THG with pCMV-lacZ (bottom row, $2^{\text {nd }}$ column). Examining these two at a higher magnification (Figures 15 - 17) reveals that empty $\mathbf{4 0 - T H G ~ i s ~ t a k e n ~ u p ~ m u c h ~ m o r e ~ t h a n ~}$ its pCMV-lacZ polyplex. This observation confirms our hypothesis that plasmid DNA actually hinders the uptake of $\mathbf{4 0 - T H G}$ polyplexes - most likely by concealing the positive surface charge. 
HeLa

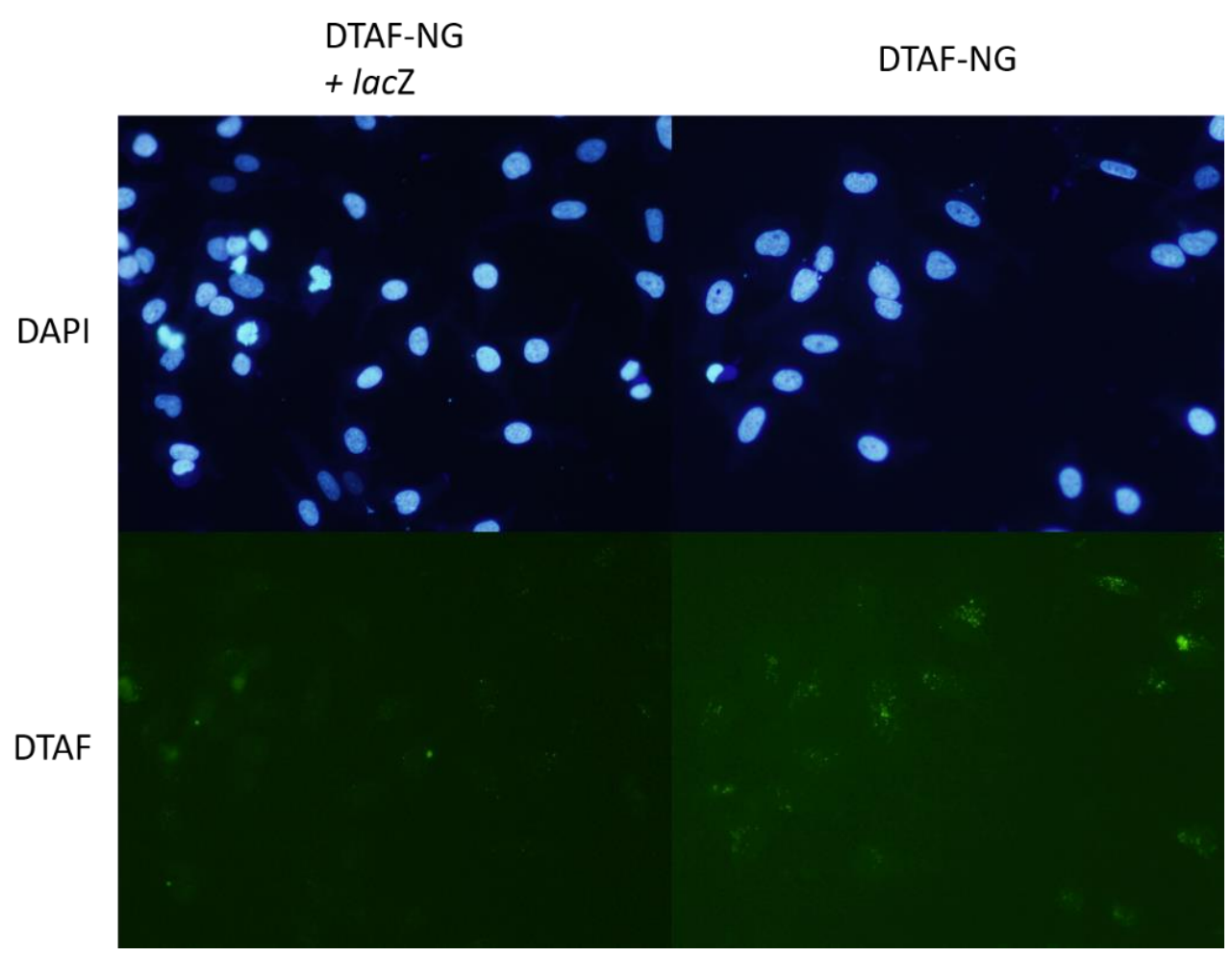

Figure 15. Fluorescence images taken of HeLa cells treated with DTAF-labelled 40-THG with or without plasmid DNA. 200x magnification. 
Chapter 6

\section{$\mathrm{C} 2 \mathrm{C} 12$}

\section{DTAF-NG}

+ lacZ

\section{DTAF-NG}

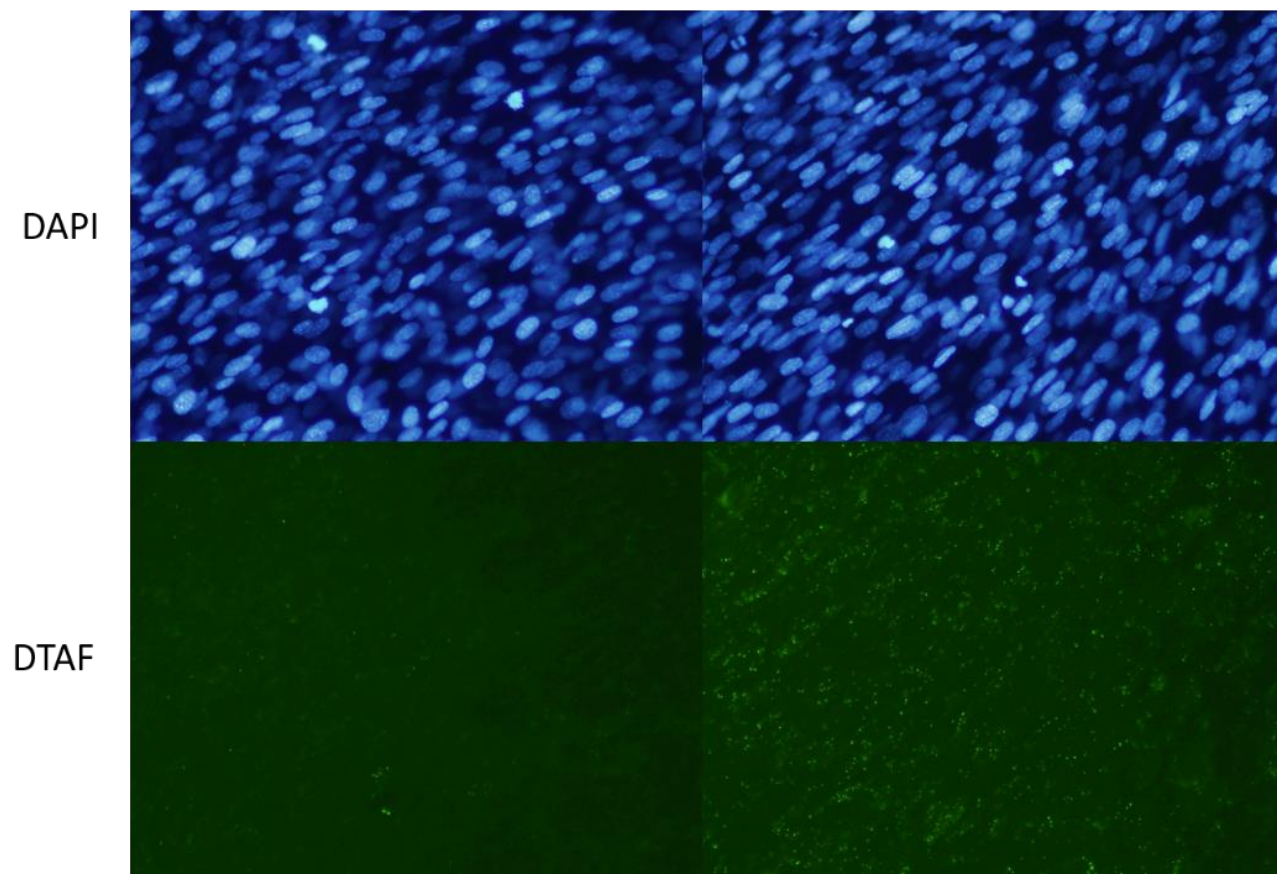

Figure 16. Fluorescence images taken of $\mathrm{C} 2 \mathrm{C} 12$ cells treated with DTAF-labelled 40-THG with or without plasmid DNA. 200x magnification. 
DTAF-NG

+ lacZ

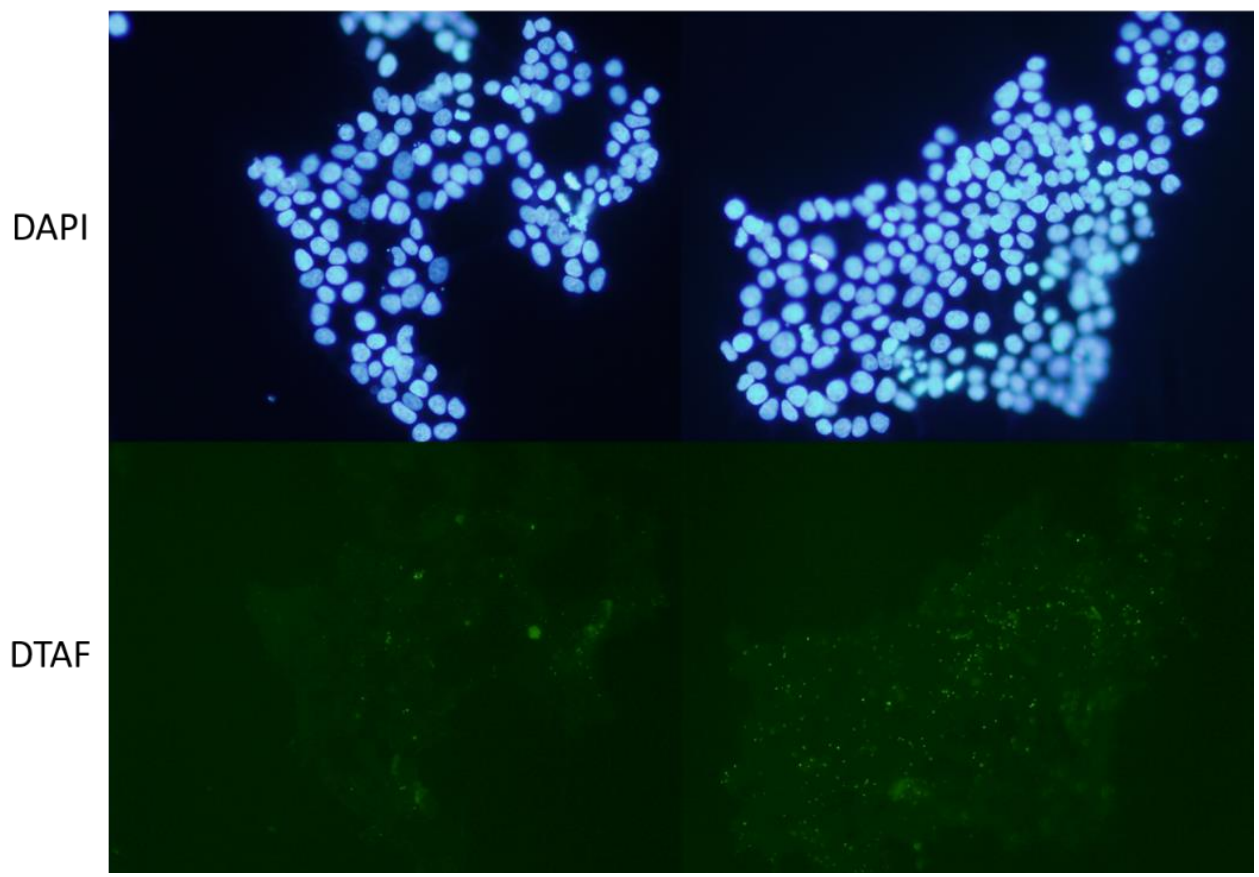

Figure 17. Fluorescence images taken of HEK293 cells treated with DTAF-labelled 40-THG with or without plasmid DNA. 200x magnification.

Nanogels containing no pCMV-lacZ exhibited higher uptake than the polyplexes, revealing endocytosis to be the obstacle hindering gene transfection. A vast majority of the polyplexes added to the cells during the experiments do not enter the cells. The few that do most likely are not enough to achieve gene transfection and express GFP fluorescence. These experiments show that for 40-THG endocytosis is the (first) major obstacle in the transfection pathway. This however does not exclude the possibility of subsequent steps also requiring optimization. 
Confocal images were taken to ensure that the green fluorescence seen in the images above arise from nanogels endocytosed by the cells and not from nanogels on the cell surface. This enables us to calculate the amount of nanogels taken up by the various cell lines more accurately.

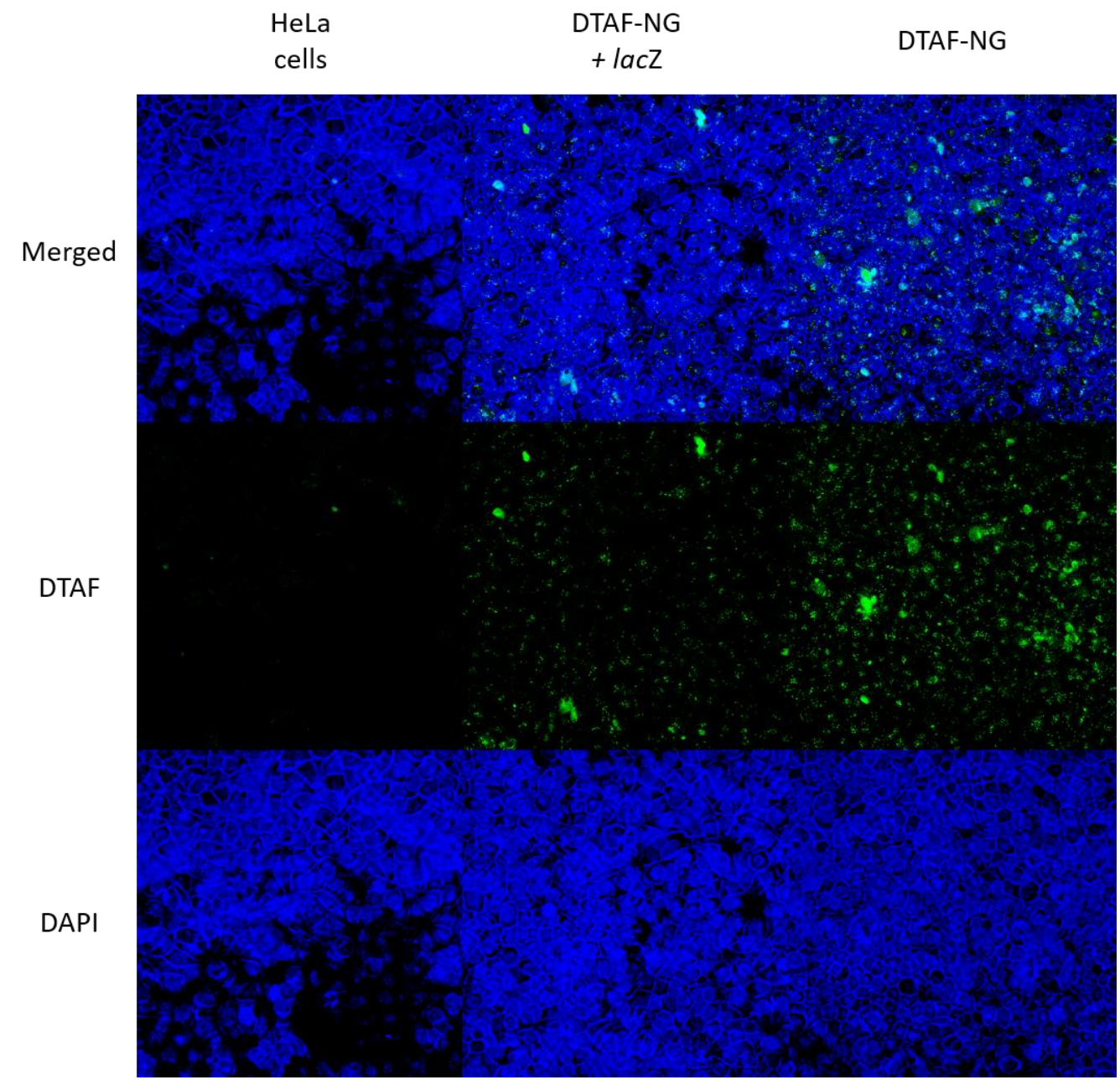

Figure 18. Confocal images were taken of HeLa cells treated with polyplexes formed from DTAF-labelled 40THG and pCMV-lacZ (middle) or with empty DTAF-labelled 40-THG (right). Examining the DTAF fluorescence emitted at $\lambda=548 \mathrm{~nm}$ reveals significantly higher fluorescence, and thus cellular uptake, for 40-THG than for pCMV-lacZ. 200x magnification. 
C2C12

cells
DTAF-NG

+ lacZ
DTAF-NG

Merged

DTAF

DAPI

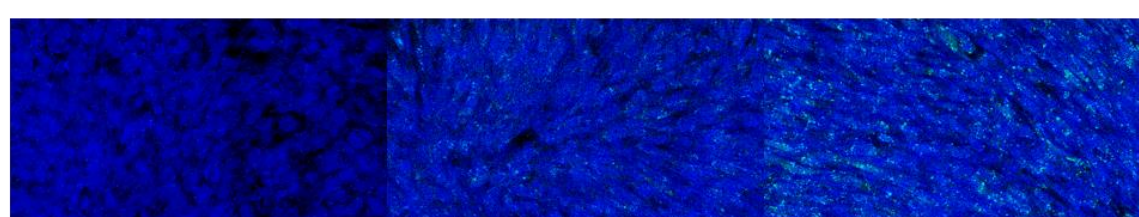




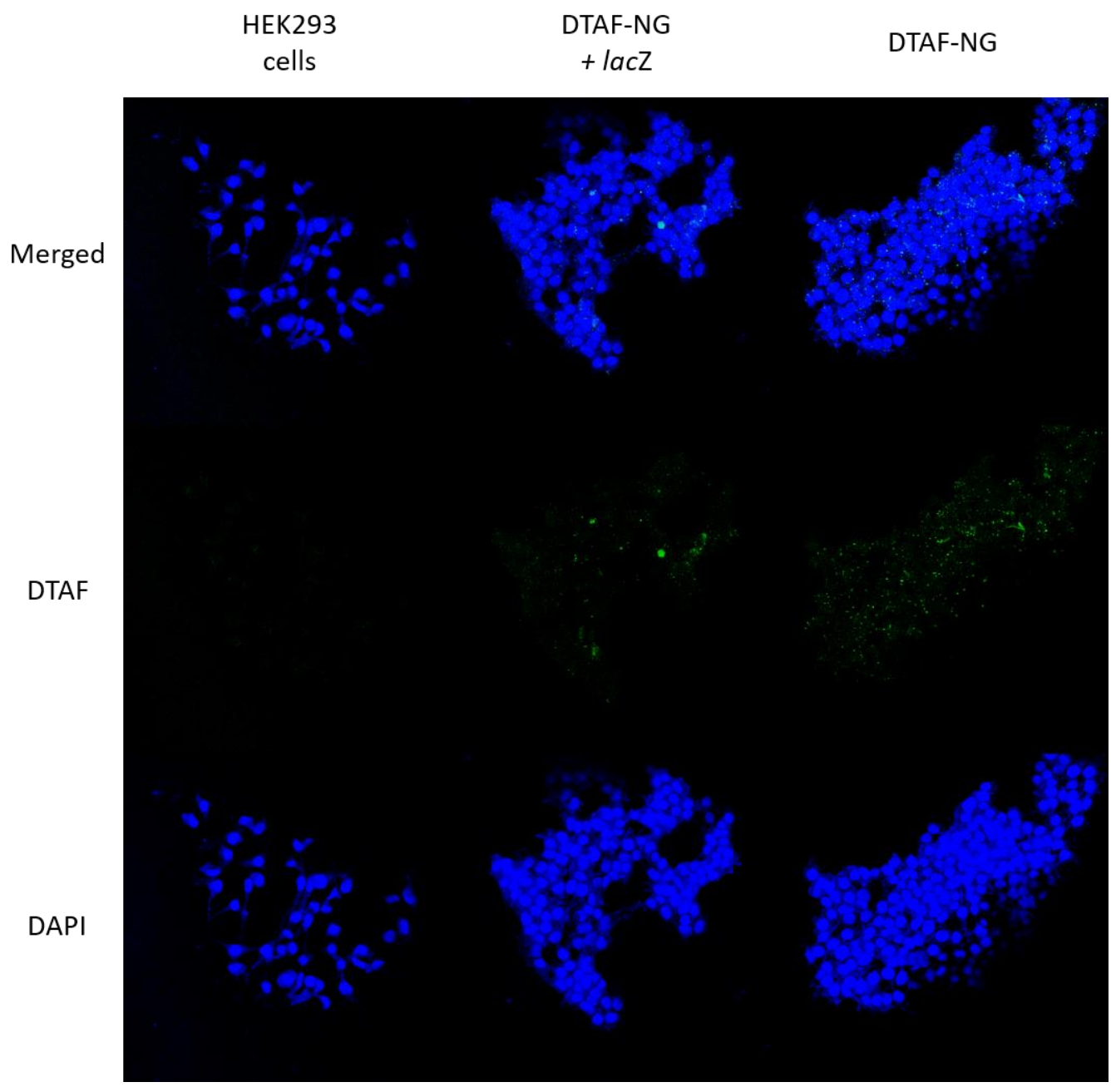

Figure 20. Confocal images were taken of HEK293 cells treated with polyplexes formed from DTAF-labelled 40-THG with pCMV-lacZ (middle) or with empty DTAF-labelled 40-THG (right). Examining the DTAF fluorescence emitted at $\lambda=548 \mathrm{~nm}$ reveals higher fluorescence, and thus cellular uptake, for 40-THG than for pCMV-lacZ. 200x magnification.

Confocal images revealed that the grand majority of the nanogels present were located inside the cells with a negligible amount residing on the cell surface. The intensity of the 
green fluorescence in the images in Figure 18 was quantified, revealing that for HeLa cells the endocytosed empty 40-THG had a 2.4 times higher fluorescence intensity, and thus uptake, than the endocytosed polyplexes. For C2C12 cells and HEK293 cells the green fluorescence intensity and uptake was respectively 12 times and 3.4 times higher for empty 40-THG than for polyplexes.

\subsection{Conclusions}

The transfection efficiency of 40-THG sulfonium nanogel was evaluated for various cell lines at varying plasmid DNA concentrations and incubation periods. Optimization of the experiments resulted in lower cell death but not in gene transfection. After trialing multiple cell lines with no success, the hypothesis that endocytosis is the bottleneck was confirmed. Comparing dyed 40-THG with dyed polyplex revealed a significantly lower uptake of pDNA-bound polyplexes rendering virtually no transfection. As such it is concluded that 40-THG is not suitable as plasmid delivery vehicle for gene therapy. Linear sulfonium polymers did exhibit some transfection for HEK293 cells suggesting a possibility of sulfonium nanogels successfully functioning as transfection vectors if sulfonium polymers are crosslinked to nanogels in the presence of plasmid DNA. In this way the DNA will be encapsulated as opposed to attached on the outer surface of the nanogel, facilitating uptake.

HEK293 cells show promise by outperforming HeLa and C2C12 cells in transfection efficiency, as expected from literature. However, due to its semi-adherent nature handling remained difficult. Although optimization to improve adherence is highly desirable, significant increase in transfection efficiency is not expected. 
Cytotoxicity experiments indicated that $\mathbf{4 0 - T H G ~ i s ~ b i o c o m p a t i b l e ~ a n d ~ n o n - t o x i c . ~ A l l ~}$ polyplexes exhibited a minimum of $90 \%$ cell viability across all cell lines, except for HEK293.

The parameter not evaluated in this work is the surface charge of the sulfonium nanogel. Increasing the surface charge could enhance uptake enough to achieve satisfactory gene transfection. This is further supported by the successful transfection seen for the sulfonium polymer with a surface charge of $+37 \mathrm{mV}$.

\subsection{Acknowledgements}

Dr. Sandra Michel-Souzy is acknowledged for her help with the biological experiments and keeping spirits high. Naomi Hamelmann is acknowledged for helping guide the experiments and giving great feedback.

\subsection{Experimental section}

Materials. All chemicals were purchased and used without further purification unless stated otherwise. Sodium hydroxide (98\%), branched polyethylene imine (bPEI $25 \mathrm{k}$, $25 \mathrm{kDa})$, HEPES (99.5\%) sodium salt, L-Glutamine solution, Penicillin-Streptomycin solution, Dulbecco's Modified Eagle Medium, high glucose (DMEM), Dulbecco's Phosphate-Buffered Saline (DPBS), Trypsin - EDTA solution, Fetal Bovine Serum (FBS), Lipofectamine $^{\mathrm{TM}} 3000$ Transfection Reagent, 4',6-diamidino-2-phenylindole (98\%, DAPI) and Resazurin Sodium Salt were purchased from Sigma Aldrich. Lipofectamine ${ }^{\mathrm{TM}} 3000$ Transfection Reagent was prepared according to provided protocol. Wheat Germ Agglutinin $\mathrm{CF}^{\oplus} 405 \mathrm{~S}$ Conjugate (WGA) was obtained from Biotium. 5-(4,6- 
Dichlorotriazinyl) aminofluorescein single isomer (5-DTAF) was purchased from Thermo Fisher. pCMV-GFP and pCMV-lacZ were purchased from the Plasmid Factory. HeLa, bEnd.3, HepG2, C2C12, RAW264, HEK293 and HFF cells were purchased from ECACC. Super folder green fluorescent protein (sfGFP) was synthesized according to literature. ${ }^{38}$

Analysis. Fluorescence microscope images were taken on an Olympus IX2-ILL 100 with 10x and 20x objective, operating at ex $350 \mathrm{~nm} / \mathrm{em} 420 \mathrm{~nm}$ for DAPI and WGA and ex 460-490 nm / em $525 \mathrm{~nm}$ for GFP and DTAF. Fluorescence was measured on an EnSight ${ }^{\mathrm{TM}}$ multimode plate reader exciting at $560 \mathrm{~nm}$ and emitting at $590 \mathrm{~nm}$. Confocal Fluorescence Microscopy (CFM) images were taken on a Zeiss Confocal LSM880 with 20x objective, exciting at $405 \mathrm{~nm}$ and emitting at $454 \mathrm{~nm}$ for DAPI and WGA. GFP and DTAF images were excited at $488 \mathrm{~nm}$ and emission was recorded at $548 \mathrm{~nm}$. The intensities were quantified in ImageJ and corrected for background signals.

Sulfonium polymers. pGMA ( 80 repeating units, $100 \mathrm{mg}, 0.68 \mathrm{mmol}$ epoxides) was dissolved in a mixture of $\mathrm{AcOH} /$ acetone $(1: 1,3 \mathrm{~mL})$. To the flask, diethyl sulfide $(0.22$ $\mathrm{mL}, 2.04 \mathrm{mmol}, 3.0$ equiv.) was added. After stirring for $24 \mathrm{~h}$, the product was precipitated twice into diethyl ether yielding a water-soluble polymer with $88 \%$ conversion. The polymer ( $0.056 \mathrm{mmol}$ epoxides) was subsequently dissolved in $10 \mathrm{~mL}$ of water and added to a snap cap vial. A stir bar, LiOH (38 mg, $1.59 \mathrm{mmol}, 3$ equiv.) and 1-thioglycerol (0.14 $\mathrm{mL}, 1.59 \mathrm{mmol}, 3$ equiv.) were added to the vial and the reaction mixture stirred for $24 \mathrm{~h}$. The polymer was then dialyzed ( $M W C O=3.5 \mathrm{kDa}$ ) against water for $3 \mathrm{~d}$, concentrated and set to $\mathrm{pH}$ 7. The addition of HEPES salt gave a polymer solution of $3.77 \mathrm{mg} / \mathrm{mL}$ in $10 \mathrm{mM}$ HEPES buffer (HEPES.NaOH, pH = 7).

${ }^{1} \mathrm{H}$ NMR (pGMA 88\% S+ $12 \%$ thioglycerol, $400 \mathrm{MHz}, \mathrm{D}_{2} \mathrm{O}$ ): $\delta$ (ppm): 3.46 (s, sulfonium $\mathrm{CH}_{2}$ ), $2.80\left(\mathrm{~s}\right.$, thioglycerol $\mathrm{CH}$ ), $2.68\left(\mathrm{~s}\right.$, thioglycerol $\left.\mathrm{CH}_{2}\right), 1.90\left(\mathrm{~s}, \mathrm{AcO}^{-}\right), 1.50$ (s, sulfonium $\mathrm{CH}_{3}$ ).

pGMA (80 repeating units, $100 \mathrm{mg}, 0.68 \mathrm{mmol}$ epoxides) was dissolved in a mixture of $\mathrm{AcOH} /$ acetone $(1: 1,3 \mathrm{~mL})$. To the flask, diethyl sulfide $(0.59 \mathrm{~mL}, 5.43 \mathrm{mmol}$, 
8.0 equiv.) was added. After stirring for $24 \mathrm{~h}$, the product was precipitated twice into diethyl ether followed by dialysis (MWCO $=3.5 \mathrm{kDa}$ ) against water for $3 \mathrm{~d}$. Freeze drying yielded $130 \mathrm{mg}$ of a water-soluble polymer with $92 \%$ conversion. The sulfonium polymer was stored in $10 \mathrm{mM}$ HEPES buffer (HEPES.NaOH, $\mathrm{pH}=7$ ) at $2 \mathrm{mg} / \mathrm{mL}$.

${ }^{1} \mathrm{H}$ NMR (pGMA 92\% S+ 8\% epoxides, $400 \mathrm{MHz}, \mathrm{D}_{2} \mathrm{O}$ ): $\delta(\mathrm{ppm}): 3.44$ (s, sulfonium $\mathrm{CH}_{2}$ ), 2.78 (s, epoxide $\mathrm{CH}$ ), 2.66 (s, epoxide $\mathrm{CH}$ ), 1.90 (s, $\left.\mathrm{AcO}^{-}\right), 1.48$ (s, sulfonium $\mathrm{CH}_{3}$ ).

Polyplex preparation. 40-THG was dissolved in 10 mM HEPES buffer solution (HEPES-NaOH, $\mathrm{pH}=7$ ). This stock solution was sterilized by means of filtration with a 200 $\mathrm{nm}$ syringe filter. In a typical procedure various amounts of stock solution, $10 \mathrm{mM}$ HEPES buffer and plasmid DNA were combined in Eppendorf tubes to give S/P ratios of 25, 50, 75, 100 and 125. The S/P ratio is the molar ratio between the sulfonium (S) atoms of 40THG and the phosphate $(P)$ atoms of the plasmid DNA. The polyplexes were incubated at RT for 10 min before use or measurements. Lipofectamine ${ }^{\mathrm{TM}} 3000$ and bPEI $_{25 \mathrm{k}}$ were utilized as positive controls. An N/P ratio of 25 was used for $\mathrm{bPEl}_{25 \mathrm{k}}$ where $\mathrm{N}$ signifies the amount of amine atoms in $\mathrm{bPEl}_{25 \mathrm{k}}$ and $\mathrm{P}$ the amount of phosphate atoms in the plasmid DNA. A stock solution was made of $\mathrm{bPEl}_{25 \mathrm{k}}$ in $10 \mathrm{mM}$ HEPES buffer solution (HEPES-NaOH, $\mathrm{pH}=7$ ) to give a concentration of $11 \mathrm{mg} / \mathrm{mL}$. This stock solution was also sterilized by means of filtration with a $200 \mathrm{~nm}$ syringe filter. Plasmid DNA ( $4 \mu \mathrm{L}$ of a $1 \mathrm{mg} / \mathrm{mL}$ solution of pCMV-GFP), bPEI ${ }_{25 k}$ stock solution $(6 \mu \mathrm{L})$ and $10 \mathrm{mM}$ HEPES buffer $(390 \mu \mathrm{L})$ were combined to form a $400 \mu \mathrm{L}$ solution of $\mathrm{bPEl}_{25 \mathrm{k}}$ polyplexes at $\mathrm{N} / \mathrm{P}=25$. The $\mathrm{bPEl}_{25 \mathrm{k}}$ polyplexes (135 nm, $+41 \mathrm{mV}$ ) were incubated at RT for $60 \mathrm{~min}$ prior to use.

Sulfonium polymers were dissolved in $10 \mathrm{mM}$ HEPES buffer solution and filtered to sterile stock solutions. Polyplexes at $\mathrm{S} / \mathrm{P}=50$ (epoxide moiety, $259 \mathrm{~nm},+37 \mathrm{mV}$ ) and $\mathrm{S} / \mathrm{P}=94$ (thioglycerol moiety, $90 \mathrm{~nm},+16 \mathrm{mV}$ ) were formed by combining stock solution, 
HEPES buffer and pCMV-GFP ( $4 \mu \mathrm{L}$ of a $1 \mathrm{mg} / \mathrm{mL}$ solution). These polyplexes were incubated at RT for 60 min prior to use.

GFP gene transfection studies. Transfection studies and cytotoxicity assays were conducted in parallel. In a typical procedure cells were seeded in a 96-well plate along with $200 \mu \mathrm{L}$ of $10 \%$ FBS containing cell culture medium supplemented with $100 \mathrm{U} / \mathrm{mL}$ penicillin, $100 \mu \mathrm{g} / \mathrm{mL}$ of streptomycin and $2 \mathrm{mM}$ of L-Glutamate $\left(\mathrm{DMEM}^{+}\right)$. Cells were incubated until a confluency of $70-95 \%$ was obtained. Prior to the transfection, medium was aspirated from the cells and replaced with $175 \mu \mathrm{L}$ of cell culture medium without FBS (DMEM-). The cells and polyplexes $\left(25 \mu \mathrm{L}\right.$ ) were incubated at $37^{\circ} \mathrm{C}$ and in $5 \% \mathrm{CO}_{2}$ and $95 \%$ humidity. Afterwards the transfection medium was aspirated and replaced by $\mathrm{DMEM}^{+}$and the cells continued incubating to allow GFP expression. The cells were then fixed and stained with WGA and DAPI to visualize the nucleus and cell membrane. Transfection efficiency was analyzed qualitatively by fluorescence microscopy and quantitatively by confocal fluorescence microscopy (CFM).

Cytotoxicity assay. Cytotoxicity assays were carried out in the same procedure as the gene transfection studies. In a typical procedure cells were seeded in a 96-well plate along with $200 \mu \mathrm{L}$ of $\mathrm{DMEM}^{+}$. Cells were incubated until a confluency of $70-95 \%$ was obtained. The medium was aspirated from the cells and replaced with $175 \mu \mathrm{L}$ of DMEMThe cells and polyplexes $(25 \mu \mathrm{L})$ were incubated at $37^{\circ} \mathrm{C}$ and in $5 \% \mathrm{CO}_{2}$ and $95 \%$ humidity. Afterwards the transfection medium was aspirated and replaced by $\mathrm{DMEM}^{+}$and the cells continued incubating to allow GFP expression. Afterwards cell viability was assessed by means of an alamarBlue ${ }^{\mathrm{TM}}$ cell viability assay. The cells were incubated with resazurin for $1 \mathrm{~h}$ followed by fluorescence measurement on a plate reader. Untreated cells (i.e. cells that were not transfected) served as positive control and were assigned $100 \%$ viable. Experiments were done in triplicate. 


\subsection{References}

1. Mingozzi, F.; High, K. A., Therapeutic in vivo gene transfer for genetic disease using AAV: progress and challenges. Nature reviews genetics 2011, 12 (5), 341-355.

2. Jean, M.; Alameh, M.; Buschmann, M.; Merzouki, A., Effective and safe genebased delivery of GLP-1 using chitosan/plasmid-DNA therapeutic nanocomplexes in an animal model of type 2 diabetes. Gene therapy 2011, 18 (8), 807-816.

3. Han, J.; McLane, B.; Kim, E.-H.; Yoon, J.-W.; Jun, H.-S., Remission of diabetes by insulin gene therapy using a hepatocyte-specific and glucose-responsive synthetic promoter. Molecular Therapy 2011, 19 (3), 470-478.

4. Won, Y.-W.; Adhikary, P. P.; Lim, K. S.; Kim, H. J.; Kim, J. K.; Kim, Y.-H., Oligopeptide complex for targeted non-viral gene delivery to adipocytes. Nature Materials 2014, 13 (12), 1157-1164.

5. Izquierdo, M., Short interfering RNAs as a tool for cancer gene therapy. Cancer gene therapy 2005, 12 (3), 217-227.

6. Park, J.; Singha, K.; Son, S.; Kim, J.; Namgung, R.; Yun, C.; Kim, W., A review of RGDfunctionalized nonviral gene delivery vectors for cancer therapy. Cancer gene therapy 2012, 19 (11), 741-748.

7. Cheng, C. J.; Bahal, R.; Babar, I. A.; Pincus, Z.; Barrera, F.; Liu, C.; Svoronos, A.; Braddock, D. T.; Glazer, P. M.; Engelman, D. M., MicroRNA silencing for cancer therapy targeted to the tumour microenvironment. Nature 2015, 518 (7537), 107110.

8. Yu, X.; Gong, L.; Zhang, J.; Zhao, Z.; Zhang, X.; Tan, W., Nanocarrier based on the assembly of protein and antisense oligonucleotide to combat multidrug resistance in tumor cells. Science China Chemistry 2017, 60 (10), 1318-1323.

9. He, Z.-Y.; Jin, Z.-H.; Zhan, M.; Qin, Z.; Li, Z.; Xu, T., Advances in quantum dotmediated siRNA delivery. Chinese Chemical Letters 2017, 28 (9), 1851-1856. 
10. Burnett, J. C.; Rossi, J. J.; Tiemann, K., Current progress of siRNA/shRNA therapeutics in clinical trials. Biotechnology journal 2011, 6 (9), 1130-1146.

11. Whitehead, K. A.; Langer, R.; Anderson, D. G., Knocking down barriers: advances in siRNA delivery. Nature reviews Drug discovery 2009, 8 (2), 129-138.

12. Petrova, N. S.; Chernikov, I. V.; Meschaninova, M. I.; Dovydenko, I. S.; Venyaminova, A. G.; Zenkova, M. A.; Vlassov, V. V.; Chernolovskaya, E. L., Carrierfree cellular uptake and the gene-silencing activity of the lipophilic siRNAs is strongly affected by the length of the linker between siRNA and lipophilic group. Nucleic acids research 2012, 40 (5), 2330-2344.

13. Jackson, L. A.; Anderson, E. J.; Rouphael, N. G.; Roberts, P. C.; Makhene, M.; Coler, R. N.; McCullough, M. P.; Chappell, J. D.; Denison, M. R.; Stevens, L. J., An mRNA vaccine against SARS-CoV-2-preliminary report. New England Journal of Medicine 2020.

14. Molla, M. R.; Levkin, P. A., Combinatorial approach to nanoarchitectonics for nonviral delivery of nucleic acids. Advanced Materials 2016, 28 (6), 1159-1175.

15. Zhao, Y.; Wang, W.; Guo, S.; Wang, Y.; Miao, L.; Xiong, Y.; Huang, L., PolyMetformin combines carrier and anticancer activities for in vivo siRNA delivery. Nature communications 2016, 7 (1), 1-9.

16. Kay, M. A., State-of-the-art gene-based therapies: the road ahead. Nature Reviews Genetics 2011, 12 (5), 316-328.

17. Jones, C. H.; Chen, C.-K.; Ravikrishnan, A.; Rane, S.; Pfeifer, B. A., Overcoming nonviral gene delivery barriers: perspective and future. Molecular pharmaceutics 2013, 10 (11), 4082-4098.

18. Zhou, J.; Liu, J.; Cheng, C. J.; Patel, T. R.; Weller, C. E.; Piepmeier, J. M.; Jiang, Z.; Saltzman, W. M., Biodegradable poly (amine-co-ester) terpolymers for targeted gene delivery. Nature materials 2012, 11 (1), 82-90. 
19. Ahn, H. H.; Lee, J. H.; Kim, K. S.; Lee, J. Y.; Kim, M. S.; Khang, G.; Lee, I. W.; Lee, H. B., Polyethyleneimine-mediated gene delivery into human adipose derived stem cells. Biomaterials 2008, 29 (15), 2415-2422.

20. Nie, X.; Zhang, Z.; Wang, C.-H.; Fan, Y.-S.; Meng, Q.-Y.; You, Y.-Z., Interactions in DNA condensation: an important factor for improving the efficacy of gene transfection. Bioconjugate chemistry 2018, 30 (2), 284-292.

21. Hemp, S. T.; Allen Jr, M. H.; Smith, A. E.; Long, T. E., Synthesis and properties of sulfonium polyelectrolytes for biological applications. ACS Macro Letters 2013, 2 (8), 731-735.

22. Hemp, S. T.; Allen Jr, M. H.; Green, M. D.; Long, T. E., Phosphonium-containing polyelectrolytes for nonviral gene delivery. Biomacromolecules 2012, 13 (1), 231238.

23. Kramer, J. R.; Schmidt, N. W.; Mayle, K. M.; Kamei, D. T.; Wong, G. C.; Deming, T. J., Reinventing cell penetrating peptides using glycosylated methionine sulfonium ion sequences. ACS central science 2015, 1 (2), 83-88.

24. Mackenzie, M. C.; Shrivats, A. R.; Konkolewicz, D.; Averick, S. E.; McDermott, M. C.; Hollinger, J. O.; Matyjaszewski, K., Synthesis of poly (meth) acrylates with thioether and tertiary sulfonium groups by ARGET ATRP and their use as siRNA delivery agents. Biomacromolecules 2014, 16 (1), 236-245.

25. Zhu, D.; Yan, H.; Liu, X.; Xiang, J.; Zhou, Z.; Tang, J.; Liu, X.; Shen, Y., Intracellularly disintegratable polysulfoniums for efficient gene delivery. Advanced Functional Materials 2017, 27 (16), 1606826.

26. Tong, L.; Chuang, C.-C.; Wu, S.; Zuo, L., Reactive oxygen species in redox cancer therapy. Cancer letters 2015, 367 (1), 18-25.

27. Boussif, O.; Lezoualc'h, F.; Zanta, M. A.; Mergny, M. D.; Scherman, D.; Demeneix, B.; Behr, J.-P., A versatile vector for gene and oligonucleotide transfer into cells in 
culture and in vivo: polyethylenimine. Proceedings of the National Academy of Sciences 1995, 92 (16), 7297-7301.

28. Yamano, S.; Dai, J.; Moursi, A. M., Comparison of transfection efficiency of nonviral gene transfer reagents. Molecular biotechnology 2010, 46 (3), 287-300.

29. Thomas, P.; Smart, T. G., HEK293 cell line: a vehicle for the expression of recombinant proteins. Journal of pharmacological and toxicological methods 2005, 51 (3), 187-200.

30. Elzes, M. R.; Si, G.; Engbersen, J. F.; Paulusse, J. M., Thiourea-functional bioreducible poly (amido amine) $\mathrm{s}$ in gene delivery. In Targeted Nanosystems for Therapeutic Applications: New Concepts, Dynamic Properties, Efficiency, and Toxicity, ACS Publications: 2019; pp 93-117.

31. McMahon, J.; Signori, E.; Wells, K.; Fazio, V.; Wells, D., Optimisation of electrotransfer of plasmid into skeletal muscle by pretreatment with hyaluronidase-increased expression with reduced muscle damage. Gene therapy 2001, 8 (16), 1264-1270.

32. Stephens, D. J.; Pepperkok, R., The many ways to cross the plasma membrane. Proceedings of the National Academy of Sciences 2001, 98 (8), 4295-4298.

33. Bureau, M.; Naimi, S.; Ibad, R. T.; Seguin, J.; Georger, C.; Arnould, E.; Maton, L.; Blanche, F.; Delaere, P.; Scherman, D., Intramuscular plasmid DNA electrotransfer: biodistribution and degradation. Biochimica et Biophysica Acta (BBA)-Gene Structure and Expression 2004, 1676 (2), 138-148.

34. Lukacs, G. L.; Lechardeur, D.; Haggie, P.; Seksek, O.; Freedman, N.; Verkman, A., Size-dependent DNA mobility in cytoplasm and nucleus. Journal of biological chemistry 2000, 275 (3), 1625-1629.

35. Vaughan, E. E.; DeGiulio, J. V.; Dean, D. A., Intracellular trafficking of plasmids for gene therapy: mechanisms of cytoplasmic movement and nuclear import. Current gene therapy 2006, 6 (6), 671-681. 
36. Hatch, M. J.; Meyer, F. J.; Lloyd, W. D., Sulfonium polymers derived from Arvinylbenzyl chloride. I. Exploratory study of the preparation and properties of the monomers and polymers. Journal of applied polymer science 1969, 13 (4), 721744.

37. Ehrenberg, L.; Hussain, S., Genetic toxicity of some important epoxides. Mutation Research/Reviews in Genetic Toxicology 1981, 86 (1), 1-113.

38. Pédelacq, J.-D.; Cabantous, S.; Tran, T.; Terwilliger, T. C.; Waldo, G. S., Engineering and characterization of a superfolder green fluorescent protein. Nature biotechnology 2006, 24 (1), 79-88. 
Chapter 6

\subsection{Supplementary data}

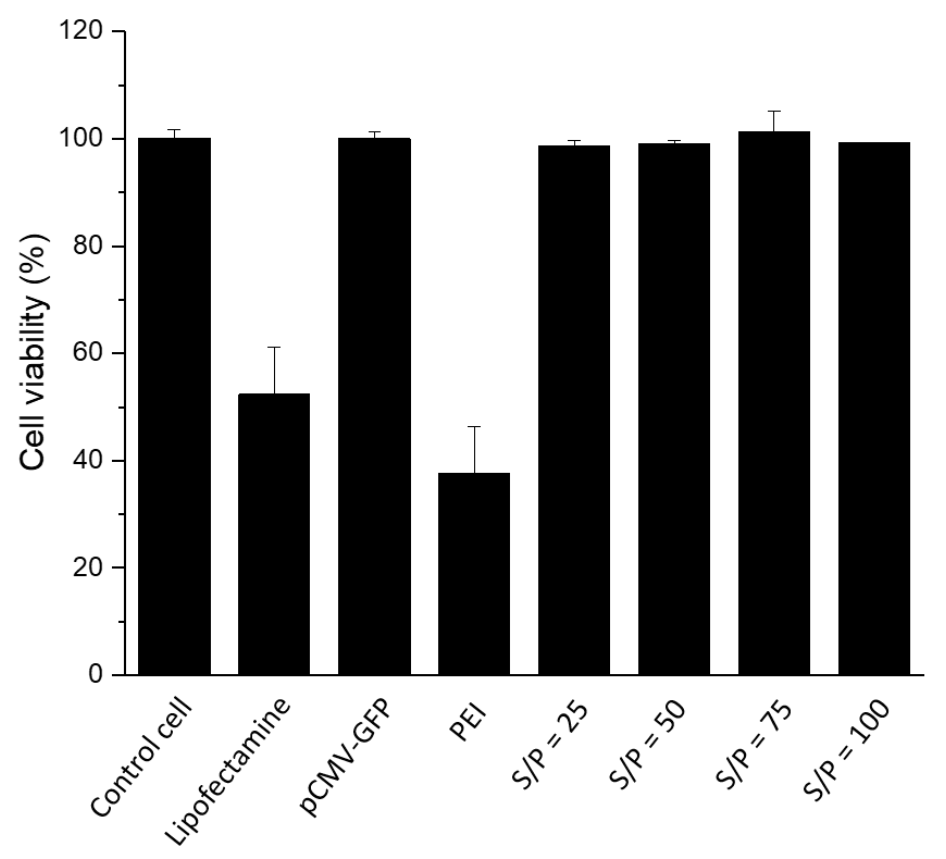

Figure S1. Cell viability of HeLa cells treated with polyplexes was evaluated by conducting an alamarBlue ${ }^{\mathrm{TM}}$ cytotoxicity experiment. Untreated cells served as negative control. Experiments were done in triplicate. 
Chapter 6

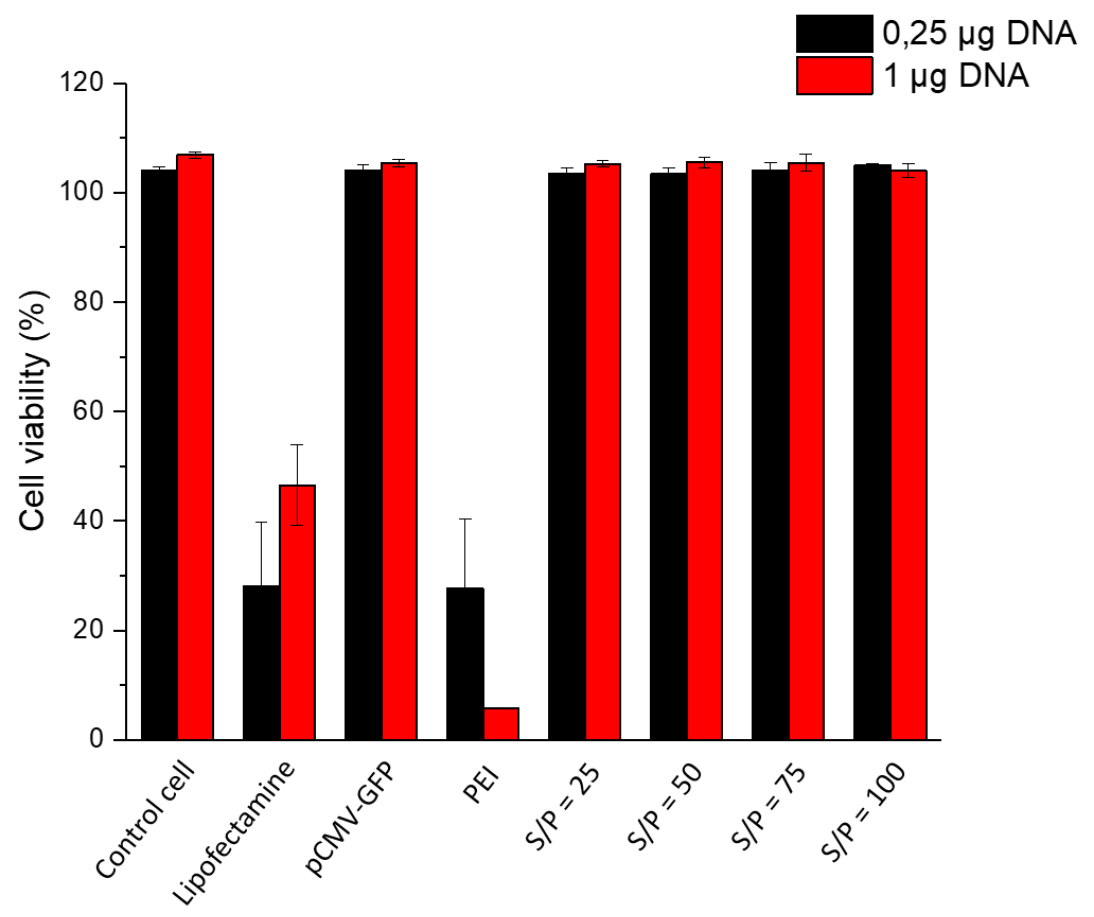

Figure S2. The cell viability of the HeLa cells treated with polyplexes is depicted above. Untreated cells served as negative control. Experiments were done in triplicate. 


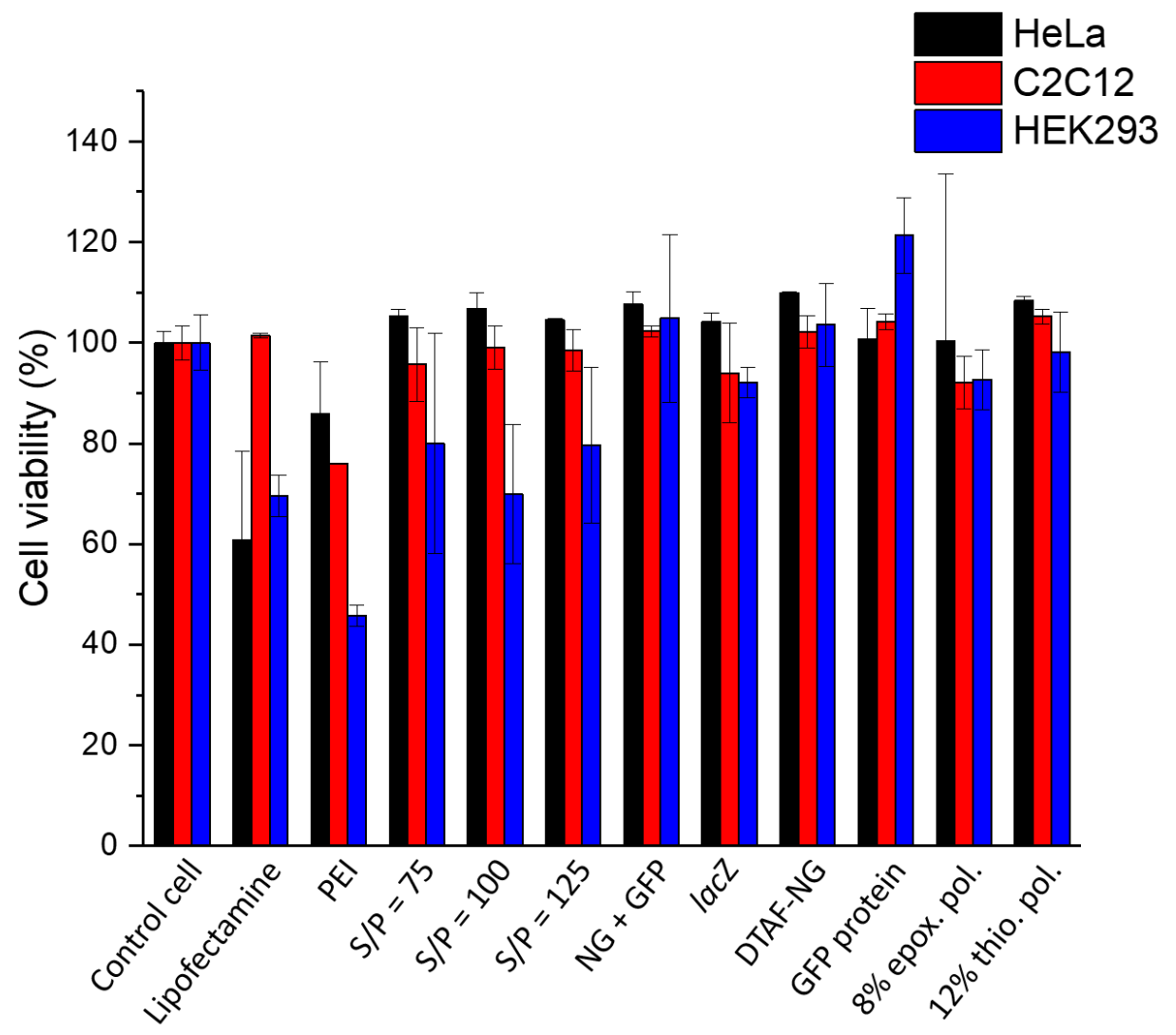

Figure S3. AlamarBlue cytotoxicity assay with resazurin gave the cell viability of the cells after $2 \mathrm{~h}$ exposure to polyplexes, DTAF-labelled 40-THG, and free GFP protein. Significant cell death is only observed for Lipofectamine 3000 and bPEI $25 \mathrm{k}$. Experiments were done in duplo. 


\section{Chapter 7}

\section{Perspectives of sulfonium nanogels in gene therapy}

In this short chapter we reflect on the work done in previous chapters. We evaluate successes achieved, the challenges met and the possibilities that remain.

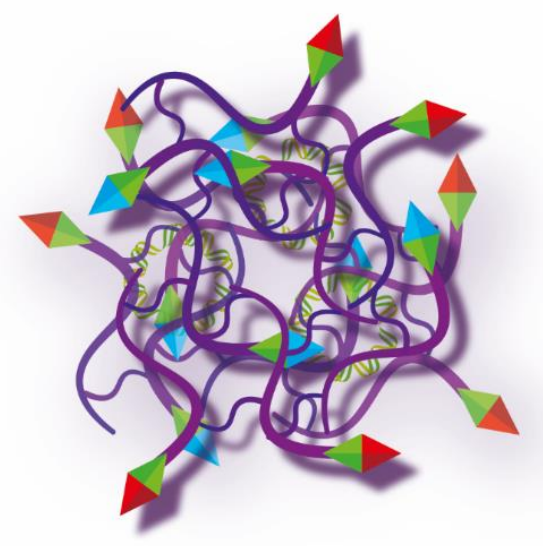




\subsection{Introduction}

As we are constantly looking for ways to modernize and improve treatments and medications for existing diseases (or new virus outbreaks) we are forced to think of new ways to treat illnesses. This can be done by improving old methods or thinking outside of the box and creating brand new concepts. Gene therapy has been around for some time and has achieved varying successes. Evidently, certain diseases would have been more deadly if certain therapeutics would have been absent. In this thesis we aimed at improving gene transfection by attempting to reduce the cytotoxicity associated with the current "gold standard" gene transfection vectors such as Lipofectamine 3000 and branched $25 \mathrm{kDa}$ polyethylene imine (PEI). In this chapter we will reevaluate the results and attempt to summarize the lessons learned based on the research described in this thesis.

\subsection{Achievements and challenges}

As discussed in Chapter 2, nanogels are very versatile and widely applicable to different fields of nanomedicine. They can be made in a variety of ways, each method with its own advantages and drawbacks. Nanogels are found in branches of medicine such as wound healing, drug delivery, pain management, vaccines and gene therapy. In the latter, cationic nanogels can efficiently transport nucleic acids into cells to be transfected or impede the expression of certain proteins. ${ }^{1-8}$ The most efficient gene transfection vectors employed some form of biodegradability to neutralize the positive charge needed to achieve sufficient cell uptake. ${ }^{9-10}$ Upon entering the cell the nanogel or polymer will deteriorate into neutral fragments to prevent cytotoxicity. 


\subsubsection{The nanogel network structure}

Our goal was the synthesis of well-defined nanogels in a single step with control over the size. This appeared successful as shown by the plots in which the hydrodynamic diameter is given as a function of monomer conversion. The nanogels exhibited great versatility and tolerability as a variety of post-polymerization modifications were possible - even sequentially on the same batch of nanogel. Barring the fact that radical polymerizations are highly sensitive, the polydispersity of the nanogels and lack of reproducibility of the sodium azide reaction and size/conversion relationship, suggest that the exact mechanism by which the nanogels are formed is not yet fully understood. A one-step reaction from mono- and divalent monomers to multiple spheres of a crosslinked network is both elegant and efficient as well as highly intricate and complicated. The conditions must be precisely tuned to achieve multiple, independent growing clusters. The use of a chain transfer agent along with fine tuning all necessary parameters greatly simplifies nanogel formation. Each nanogel network starts out as monomers reacting with each other, forming branched polymers at roughly the same time. As the reaction proceeds, no new clusters are formed and the existing polymer branches undergo intramolecular crosslinking, forming nanogels. The polymer chemists who developed this method have provided protocols to achieve a great level of control. ${ }^{11-13}$ However, the ideal situation described above is not attainable. Dynamic light scattering measurements tell us approximately when branched polymers become nanogels and that their hydrodynamic radius increases as the reaction proceeds. DLS measurements also tell us when individual nanogels react with each other forming aggregates and macrogels. Nanogels presumably grow from multiple sites on its network simultaneously. However, it is not known if all active sites have the same reactivity and whether or not it varies throughout the synthesis. It is plausible that some methacrylate ends, such as those in the center of the nanogel, are less or no longer accessible by monomers during the polymerization 
reaction. The uneven growth would lead to non-uniform, heterogeneous constructs. Along with fluctuations within the same nanogel cluster, the question also remains in what capacity individual nanogels from the same batch vary from each other. The high polydispersity seen on TEM along with varying solubility of nanogels from the same batch, would indicate significant differences. It is my belief that theoretical models and computational experiments in combination with kinetics and fragmentation studies will elucidate the composition of the nanogel and clarify the mechanism by which it is made. As those experiments are out of the scope of this thesis the choice was made to focus on surface functionalizations of nanogels to form useful and versatile products for biomedical applications.

\subsubsection{One-step crosslinking polymerization to nanogels}

Alternatively, more insight and control over the nanogel synthesis, structure and composition can be achieved in return for efficiency, by converting to a two- or more step synthesis. The one-step synthesis method used in this thesis elegantly showcases the level of control and creativity achievable in radical polymerizations. As seen in Figure 1 below, the nanogels experience an exponential growth at the start of the reaction followed by a period of linearity. As the reaction proceeds, nanogel growth levels off and reaches a plateau. Reactions carried out at a higher combined monomer concentration do not plateau, but form a macrogel. 


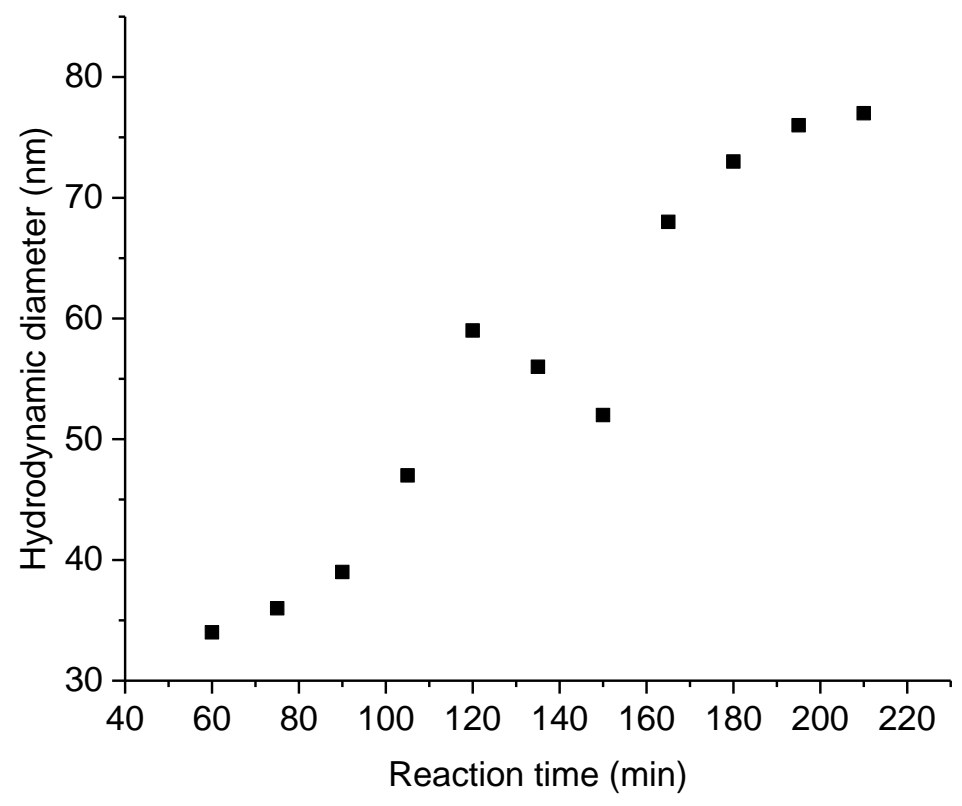

Figure 1. GMA and EGDMA were reacted together to form GMA-EGDMA nanogels at a combined monomer (both monovinyl and divinyl) concentration of $4 \%$ in 1,4-dioxane. The hydrodynamic diameters of the nanogels were measured on DLS and are depicted above as a function of reaction time.

Figure 1 not only highlights the level of control achievable with this synthesis protocol but also its sensitivity. The reaction mixture was homogenized in a flask, distributed evenly between identical glass ampoules, degassed ( $\max 5$ ampoules at a time) by means of 3 freeze-pump-thaw cycles and flame sealed. All ampoules were added to an oil bath at $70^{\circ} \mathrm{C}$ simultaneously and quenched one by one with $0.5 \mathrm{~mL}$ DCM every 15 minutes. Despite the precautions taken, two out of eleven data points were outliers; most likely due to minute differences during handling, degassing, stirring or heating. The choice for this one-step crosslinking polymerization protocol was motivated by the requirement of size control in biomedical applications. The ideal size of nanoparticles destined for the 
human body is $10-200 \mathrm{~nm} \cdot{ }^{14-15}$ Depending on its target organ or tissue a smaller, more precise size scope is desired. However, the full $10-200 \mathrm{~nm}$ range most likely is not required. Likewise, the ability to synthesize a large number of nanogels in a one-pot reaction, each with a different size, is an exceptionally efficient feature that unfortunately will go unused. In most biomedical studies a handful of samples is enough. Li et al. first synthesized poly(glycidyl methacrylate) (pGMA) followed by ring-opening amination with ethylenediamine and then crosslinked to a nanogel by amidation with $\alpha$-lipoic acid. ${ }^{8}$ Although this synthesis route includes 3 steps to form a nanogel, the existence of intermediate linear polymers facilitates the calculation of the molecular weight, polydispersity and crosslink density of the nanogel. The precision provided by such a protocol outweighs the efficiency of a one-step nanogel synthesis - especially if only a couple of distinct nanogel sizes are needed.

\subsubsection{Nanogel properties}

A feature we have long been interested in is the density of the nanogel. TEM measurements, surprisingly, taught us that the density increases with conversion, which contradicted our former hypothesis. The crosslink density and the molecular weight of the nanogels can be estimated with GPC and NMR but not with sufficient accuracy. A DLS setup which includes a MALLS detector would provide the molecular weight of the nanogel, along with useful information regarding its density. Such a setup however was not available.

As witnessed in Chapters 3 and 4, the surface conjugations on the nanogel were well tolerated and the new functional groups remained accessible. Sulfonium nanogels were synthesized in a single step from glycidyl methacrylate nanogels. The nanogels were stable for up to two weeks after which a reduction was seen in surface charge, most likely 
due to hydrolysis of the sulfonium moiety. Sulfonium nanogels successfully bound pDNA through electrostatic interactions forming polyplexes. The polyplexes were stable for some hours after which dissociation occurred.

\subsubsection{Sulfonium nanogels for gene transfection}

Transfection experiments were performed on various cell lines under varying conditions with no success. The sulfonium nanogel carried a positive surface charge which was shielded by pDNA when complexed, hindering sufficient polyplex uptake. As the nanogels induced only a bare minimum of transfection it would be fair to conclude that sulfonium nanogels cannot serve as transfection vectors. However, potential improvements still remain. The sulfonium nanogel had a surface charge of $+14 \mathrm{mV}$ which is lower than those usually reported in literature. ${ }^{8,16-17}$ Increasing the surface charge could produce tighter bonds between the nanogel and pDNA and as well as higher multivalent interactions between the polyplex and the cell membrane. This in turn would increase the cellular uptake of the polyplexes. Secondly, nanogels are large spherical particles that most likely bind pDNA on their outer surface, as opposed to an inner cavity or coiling together like linear polymers do. As such the nanogel provides minimal to no protection of the plasmid along with little hold. This is further suggested by the high S/P ratios needed to achieve proper binding and the timeframe in which polyplex dissociation starts. Others have shown that it is possible to synthesize the nanogel in the presence of the pDNA to ensure its encapsulation in the matrix of the nanogel. ${ }^{5}$ Although this method is worth testing, it is unlikely that the pDNA will survive the multiple synthesis and purification steps required to synthesize sulfonium nanogels. Another alternative entails exploiting the swelling and shrinking behavior intrinsic to nanogels to load pDNA into its matrix. It is also possible to synthesize sulfonium nanogels with a lower crosslink density resulting in a more open 
nanogel construct, better able at encapsulating pDNA in its inner pockets. The transfection experiments carried out in Chapter 6 were performed as a proof of principle. Therefore certain characterization steps such as gel electrophoresis and determination of the proton sponge effect and buffering capacity were omitted. As gene transfection was not successful, all characterization steps should be performed in following experiments or when repeating the study to gain as much insight as possible.

\subsection{Outlook}

The broad versatility, tunability and applicability of nanogels remains evident. In this thesis we demonstrated that sequential reactions on the same batch of nanogel are well tolerated, with minimal changes in size or surface charge, provided that the nanogels are still stable and not aggregating or hydrolyzing. As of yet it remains unknown if sulfonium nanogels can effectively function as gene vectors. Although many conditions were trialed during the transfection experiments, possibilities for optimization remain. Sulfonium nanogels are susceptible to hydrolysis meaning they should either be stabilized or used within a week of synthesis. The influence of the nanogel surface charge appears quite significant; that of polyplex size not as much, as of yet. It is thus good practice to conduct transfection experiments on polyplexes baring various amounts of $\zeta$-potential, with focus on the higher charges. Nonetheless, the field of sulfonium polymer gene vectors is still in its infancy, meaning that statements made at this time should be regarded as tentative.

\subsection{References}

1. Zhao, N.; Lin, X.; Zhang, Q.; Ji, Z.; Xu, F. J., Redox-triggered gatekeeper-enveloped starlike hollow silica nanoparticles for intelligent delivery systems. Small 2015, 11 (48), 6467-6479. 
2. Xu, F.; Zhu, Y.; Chai, M.; Liu, F., Comparison of ethanolamine/ethylenediaminefunctionalized poly (glycidyl methacrylate) for efficient gene delivery. Acta biomaterialia 2011, 7 (8), 3131-3140.

3. Xu, F.; Chai, M.; Li, W.; Ping, Y.; Tang, G.; Yang, W.; Ma, J.; Liu, F., Well-defined poly (2-hydroxyl-3-(2-hydroxyethylamino) propyl methacrylate) vectors with low toxicity and high gene transfection efficiency. Biomacromolecules 2010, 11 (6), 1437-1442.

4. Dou, X.; Chai, M.; Zhu, Y.; Yang, W.; Xu, F., Aminated poly (glycidyl methacrylate) $\mathrm{s}$ for constructing efficient gene carriers. ACS applied materials \& interfaces 2013, $5(8), 3212-3218$.

5. Dimde, M.; Neumann, F.; Reisbeck, F.; Ehrmann, S.; Cuellar-Camacho, J. L.; Steinhilber, D.; Ma, N.; Haag, R., Defined pH-sensitive nanogels as gene delivery platform for siRNA mediated in vitro gene silencing. Biomaterials science 2017, 5 (11), 2328-2336.

6. Park, J. S.; Yi, S. W.; Kim, H. J.; Kim, S. M.; Shim, S. H.; Park, K.-H., Sunflower-type nanogels carrying a quantum dot nanoprobe for both superior gene delivery efficacy and tracing of human mesenchymal stem cells. Biomaterials 2016, 77, 1425.

7. Park, J. S.; Yi, S. W.; Kim, H. J.; Park, K.-H., Receptor-mediated gene delivery into human mesenchymal stem cells using hyaluronic acid-shielded polyethylenimine/pDNA nanogels. Carbohydrate polymers 2016, 136, 791-802.

8. Li, R.-Q.; Wu, W.; Song, H.-Q.; Ren, Y.; Yang, M.; Li, J.; Xu, F.-J., Well-defined reducible cationic nanogels based on functionalized low-molecular-weight PGMA for effective pDNA and siRNA delivery. Acta biomaterialia 2016, 41, 282-292.

9. Nishimura, T.; Yamada, A.; Umezaki, K.; Sawada, S.-i.; Mukai, S.-a.; Sasaki, Y.; Akiyoshi, K., Self-assembled polypeptide nanogels with enzymatically 
transformable surface as a small interfering RNA delivery platform. Biomacromolecules 2017, 18 (12), 3913-3923.

10. Zhu, D.; Yan, H.; Liu, X.; Xiang, J.; Zhou, Z.; Tang, J.; Liu, X.; Shen, Y., Intracellularly disintegratable polysulfoniums for efficient gene delivery. Advanced Functional Materials 2017, 27 (16), 1606826.

11. Isaure, F.; Cormack, P. A.; Graham, S.; Sherrington, D. C.; Armes, S. P.; Bütün, V., Synthesis of branched poly (methyl methacrylate) s via controlled/living polymerisations exploiting ethylene glycol dimethacrylate as branching agent. Chemical communications 2004, (9), 1138-1139.

12. Wang, W.; Zheng, Y.; Roberts, E.; Duxbury, C. J.; Ding, L.; Irvine, D. J.; Howdle, S. M., Controlling chain growth: a new strategy to hyperbranched materials. Macromolecules 2007, 40 (20), 7184-7194.

13. Rosselgong, J.; Armes, S. P.; Barton, W.; Price, D., Synthesis of highly branched methacrylic copolymers: observation of near-ideal behavior using RAFT polymerization. Macromolecules 2009, 42 (16), 5919-5924.

14. Venturoli, D.; Rippe, B., Ficoll and dextran vs. globular proteins as probes for testing glomerular permselectivity: effects of molecular size, shape, charge, and deformability. American Journal of Physiology-Renal Physiology 2005, 288 (4), F605-F613.

15. Ogawara, K.-I.; Yoshida, M.; Furumoto, K.; Takakura, Y.; Hashida, M.; Higaki, K.; Kimura, T., Uptake by hepatocytes and biliary excretion of intravenously administered polystyrene microspheres in rats. Journal of drug targeting 1999, 7 (3), 213-221.

16. Hong, C. A.; Kim, J. S.; Lee, S. H.; Kong, W. H.; Park, T. G.; Mok, H.; Nam, Y. S., Reductively Dissociable siRNA-Polymer Hybrid Nanogels for Efficient Targeted Gene Silencing. Advanced Functional Materials 2013, 23 (3), 316-322. 
17. Leber, N.; Nuhn, L.; Zentel, R., Cationic nanohydrogel particles for therapeutic oligonucleotide delivery. Macromolecular bioscience 2017, 17 (10), 1700092. 
Chapter 7

$240 \mid P$ a g e 


\section{Summary}

The main goal of science has always been to better understand the world we live in. By studying our surroundings we can make better sense of the world around us. With the discovery of diseases came the need to understand and cure them. Science and medicine have evolved through time leading to various medical fields and research methodologies. In the past decade scientists have been focusing on utilizing macromolecules of the nanometer size range to optimize existing treatments or develop new ones. This field of nanomedicine comprises drug and gene delivery, bio-imaging, bio-sensing and tissue engineering. Actively delivering therapeutics to their destination as opposed to relying on their passive accumulation at receptor sites result in higher effectiveness. In gene therapy illnesses caused by modifications in the human genome are treated by silencing the gene(s) responsible for the disease, introducing a missing gene or repairing a faulty one. Oligonucleotides are quite fragile, thus requiring protection during their transport. In this thesis we synthesized a crosslinked polymer network to serve as a protective gene carrier. Chapter 3 described the synthesis of GMA-EGDMA nanogels that contain a reactive epoxide moiety capable of undergoing post-polymerization reactions. GMA-EGDMA nanogels are not only versatile, their diameter is directly correlated to the monomer conversion. This synthesis method thus provides the chemist with great control over the size and functionality of the nanogel. Epoxides moieties on a nanogel remain reactive and accessible as proven by reactions with sodium azide, TFA, Boc-aminoethanethiol and dimethylethanolamine. In Chapter 4, GMA-EGDMA nanogels were given a cationic charge in order to bind anionic DNA. The choice was made for sulfonium as the positively charged moiety as opposed to the more commonly used ammonium. To render the sulfonium nanogels water-soluble, secondary reactions were done on the remaining epoxides after 
ring opening with diethylsulfide. When these reactions did not result in appreciable water-solubility, EGDMA crosslinker was substituted by a more hydrophilic equivalent. In Chapter 5 we described these water-soluble sulfonium nanogels in more detail focusing on their binding of plasmid DNA. 40-THG and 50-THG contained a thioglycerol moiety to enhance biocompatibility and hydrophilicity. As 50-THG required a lower S/P ratio to properly bind plasmid DNA, it was chosen over 40-THG to serve as a gene vector in transfection experiments, as described in Chapter 6. A variety of cell lines, uptake and transfection time and concentrations were tested with no successful transfection. We suspected the reason for the failed transfection being the stability of the polyplexes, the surface charge of the nanogels or the nanogel construct itself. A final experiment comparing cell uptake of nanogels with cell uptake of polyplexes revealed that anionic pDNA on the surface of nanogels masked their cationic surface charge, hindering proper interaction with the cell membrane and thus minimal uptake. At first glance it seems evident that sulfonium nanogels do not function as gene vectors as multiple experiments were conducted without any apparent transfection. However, as reflected in Chapter 7, although many gene transfection assays were conducted, the study as a whole was executed in a proof-of-principle manner. All polyplexes were formed from the same nanogel with a relatively low surface charge compared to known transfection vectors. As such, a more accurate conclusion would be that further experiments are required to definitively state the transfection efficiency of sulfonium nanogels. 


\section{Samenvatting}

Wetenschap heeft als primaire doel de wereld om ons heen beter te begrijpen door onze omgeving te bestuderen en kwantificeren. Sinds de ontdekking van ziektes en aandoeningen streeft men ernaar om deze beter in kaart te brengen en geneesmiddelen voor te ontwikkelen. De wetenschap en geneeskunde hebben zich door de jaren heen ontwikkeld tot verschillende medische richtingen en onderzoekstrategieën. In de laatste decennium hebben wetenschappers zich bezig gehouden met het toepassen van macromoleculen van de nanometer schaal om bestaande ziektebestrijdingstechnieken te optimaliseren of nieuwe te ontwikkelen. Dit gebied van nanogeneeskunde omvat medicijn- en genafgifte, bio imaging, biosensing en weefsel constructie. De effectiviteit van farmaceutische nanodeeltjes wordt verhoogd als zij actief getransporteerd worden naar hun doel, in plaats van passief accumuleren bij receptoren. Gen therapie tracht ziektes te genezen die zijn ontstaan door fouten in de genoom door middel van genuitschakeling, introduceren van ontbrekende genen of een defect gen te repareren. Oligonucleotiden zijn zeer fragiel en vereisen dus bescherming tijdens hun transport. In deze scriptie hebben wij een polymeer netwerk ontworpen die optreedt als beschermende gen transportmiddel. Hoofdstuk 3 beschrijft de synthese van GMAEGDMA nanogels die reactieve epoxide groepen bezitten en daardoor vervolg reacties kunnen ondergaan na polymerisatie. De GMA-EGDMA nanogels zijn niet alleen veelzijdig, hun diameter wordt bepaald door de conversie van de monomeren. Met behulp van deze methode heeft de chemicus dus veel controle over de grootte en functionaliteit van de nanogel. De epoxide groepen aan de nanogel blijven bereikbaar en reactief zoals bewezen met reacties met natrium azide, TFA, Boc-aminoethanethiol en dimethylethanolamine. In hoofdstuk 4 hebben we GMA-EGDMA nanogels voorzien van een kationische lading door 
middel van sulfonium, om het binden van anionische DNA te vergemakkelijken. Om de sulfonium nanogels wateroplosbaar te maken, werd een secundaire epoxideopeningsreactie uitgevoerd op de overgebleven expoxides. Omdat de nanogels alsnog niet voldoende wateroplosbaar waren, werd EGDMA crosslinker vervangen door een meer hydrofiele variant. In hoofdstuk 5 hebben wij deze wateroplosbare sulfonium nanogels in meer detail beschreven, waarbij we ons hebben gefocust op hun bindingsaffiniteit met DNA. Sulfonium nanogels 40-THG en 50-THG bevatten een thioglycerol groep om meer hydrofiel en biocompatibel te zijn. De keuze voor 50-THG in vervolg experimenten was gemotiveerd door de lagere S/P ratio die deze nanogel vereist om pDNA fatsoenlijk te binden. In hoofdstuk 6 werden de transfectie studies met 50-THG als transportmiddel uitgelicht. Meerdere cellijnen, opname-, transfectietijden en concentraties waren getest zonder succesvolle transfectie. Wij vermoedden dat de reden hiervoor lag bij de stabiliteit van de polyplexen, hun oppervlaktelading of de nanogel constructie zelf. In een vervolg reactie werd de celopname van polyplexen vergeleken met die van nanogels. Hieruit bleek dat de anionische lading van de DNA op de oppervlakte van de nanogels hun kationische lading vrijwel geheel maskeerde. Het gevolg hiervan is dat de nanogel geen goede interactie met de celmembraan heeft en deze dus niet voldoende opgenomen kan worden. Door de vele experimenten uitgevoerd in hoofdstuk 6 zonder enige transfectie zou men kunnen concluderen dat sulfonium nanogels niet kunnen functioneren als vervoermiddel in gentherapie. Echter, zoals beschreven in hoofdstuk 7, de transfectie studies waren uitgevoerd in een proof-of-principle wijze. Alle polyplexen waren afkomstig van één enkele nanogel met relatief lage oppervlaktelading vergeleken met gangbare transfectievectoren. Verdere onderzoek is dus nodig om definitief vast te stellen of sulfonium nanogels dienen als transfectievector. 


\section{Acknowledgements}

It has been a crazy 4.5 years for me in Enschede and at the University of Twente. I've experienced great highs and many lows. At times I did not believe I would ever finish. Thankfully I did, but not without the help of some amazing human beings, and a couple of cute doggies of course!

First and foremost Finn, Bowie, Boet, Lynn, Kimbo, Chiquita, Muffin and Lizzie. Whoever said money can't buy happiness has never owned a dog. Blessed are the ones who've experienced the unconditional love of a dog. Thank you for the kisses, the body slams, the runs, the swimming, the walkies, the wet noses, the laughs and the unconditional love.

Ook al heb je eerder gezegd dat het niet nodig is, Jeroen, ik wil je toch nog een keer bedanken voor de begeleiding en vooral het feit dat je mij serieus nam. Ook al kwam ik soms bij jou op kantoor en kon ik amper uit mijn woorden komen, ik vertrok altijd met het gevoel dat ik gehoord werd. To my committee members, chairman, academic staff (present and past) and supporting staff, Frederik, Julieta, Marleen, Andreas and Jurriaan thank you for your contributions, no matter how big or small, to my time as a PhD candidate, my dissertation and the scientist I am today. Nicole Haitjema, Nicole Katier, Izabel, Bianca, Regine, Marc Ankoné en Richard. Bedankt voor alles wat jullie niet alleen voor mij hebben gedaan maar ook alle andere aio's en studenten. Marcel, ik ben nooit eerder een technician tegengekomen die het oprecht leuk vindt om anderen te helpen zoals jij. Ik ben je eeuwig dankbaar. Michel en Clemens hartstikke bedankt voor jullie hulp met de AFM en GPC! Jullie hebben het allebei druk genoeg en konden makkelijk nee zeggen maar hebben toch gekozen om mij te helpen. Anzar and Taejun, thank you for your help with the epoxides and the lovely time in South Korea. I was only with you guys for a short month but it was honestly a highlight of my PhD. 
Min, Rachel, Leonie, Jacopo, Anuradha, Bas, Laura, Diana, Jonathan and the friendly newcomers in the chicken coop. Thank you for the laughs and good times. Jan-Willem, I've always admired your decision to return to university. The best of luck with finishing your PhD. Remí, Dhanya, Jenny, Hasnaa, Julien, Anamarija, Daniele, Candelaria, Almudena, Muhabbat, Maaike. One of the hardest lessons I had to learn during my PhD is that the people you enjoy being around the most are usually the first to leave. You guys made my time at the UT so much more enjoyable. Almu, Alberto and Cande, hearing you guys speak Spanish always comforted me and made me feel safe and at home. Na een vreselijke eerste student was ik doodsbang om weer studenten te begeleiden. Thijs, gelukkig was je echt het allerbeste student die iemand had kunnen wensen. Je had als master student alle kwaliteiten van een goede PhD student. Bedankt voor hoofdstuk 4 en heel veel succes in Eindhoven!

Luca, you were always the one person I could count on to believe me and my experiences. Not once did you try to diminish or dismiss anything I went through. I truly admire your passion for the things you believe in. As a black woman who's lived in Holland for 14 years and has learned to soften the edges of her identity to stick out less, seeing you being your unapologetic true self is absolutely beautiful. Sandra, thank you for being so accepting of me and my coping mechanisms. You have no idea how much that means to me and how safe you made me feel. I admire you so much for your helpful and joyful nature. Thank you for putting so much time and effort into my transfection studies. I literally would not be here without you. My beautiful work wife Naomi, you have been my rock and my constant throughout this whole PhD. Thank you for always being there for me, always offering help and a shoulder to cry on. I'm so incredibly lucky to have had you with me. There's no one else I would rather have by my side during my defense.

Dr. Melissa Cianfrini, Mel, I went to Thailand for a month with no intentions of making any friends. To my surprise not only did I make an amazing friend, I met a recent $\mathrm{PhD}$ 
graduate. Thank you for the wisdom and guidance and for all the online therapy sessions. I wish you all the joy and love in the world with your family. Kaaaaaaaaa my loveeeeee.

Nifi, Amely, Angie, Kétricha, Tisha, Quinlan, Adrie. No matter what type of support I get from others around me, there's nothing more healing and peaceful than being around people I don't have to explain anything to. Those trips to Utrecht, no matter how few and far between, soothed my soul and kept me going. Marc thank you for always being $100 \%$ in my corner and my biggest cheerleader. My very best friend Vanessa, thank you for accepting me as I am, along with my "unique" form of friendship. Your unwavering support and understanding has been nothing short of amazing. Thank you for always checking in; even when I get caught up in my own world and forget to ask you about yours. Thank you. And as you already know, I'm so incredibly proud of you!

Mijn lieve ten Elshof schoonfamilie. Ik had niet verwacht dat ik tijdens mijn PhD een relatie zou krijgen en laat staan zo'n lieve schoonfamilie erbij. In het begin hadden jullie mij vaak gewaarschuwd dat het bij jullie best druk kan zijn. Maar voor een Curaçaose die haar familie enorm mist was die drukte precies wat ik nodig had. Derek i Margay, danki pa mi subrina bunita Myah, ku a nase riba mi aña. Mi ta stima wak bosnan famia. Derek, mi ta hopi orguyoso di bo i tur loke bo a logra na Hulanda. Famia Luneta i famia Kusmus, masha danki pa tur orashon i tur steun ku m'a haña di bosnan tur. Mi ta inkrehiblemente kontentu ku e famia bunita, loko i uní ku mi tin. I'm so blessed to have you.

Liefie, Ramon. Je hebt een relatie gekregen met een burnt-out PhD student die ook nog eens uit een heel ander cultuur komt en meer dan 10 jaar geen relatie heeft gehad. Bedankt voor je begrip, je steun en vooral voor je geduld. Na zo lang in Nederland in mijn eentje was het wel even wennen om ineens plaats te maken in mijn leven voor een ander. En niet zomaar iemand, maar iemand wiens lichaam om de week uit elkaar valt en niet langer dan 5 minuten in de zon kan zijn. Bedankt voor je begrip als ik soms bij mijn 
vrienden of familie wil zijn en geen Nederlands wil praten. Bedankt voor je begrip elke keer dat ik de telefoontjes van mijn moeder opneem, ongeacht de tijd of waar we op dat moment mee bezig zijn. Onze relatie heeft niet altijd zekerheid gekend, maar één ding waar ik wel zeker van ben is hoeveel ik van jou houd en jij van mij.

Mama, Papa, Sanin. I have missed so many birthdays, anniversaries, random car rides and days at the beach. You guys are my foundation, my source of strength, courage and determination. No matter what I encountered during the past 4.5 years I could always handle it because I'm so incredibly loved by you three. I am nothing without my family's love. Thank you for the unwavering and unconditional support. Sanin, although you are my little brother I look up to you so much. Whenever things got tough and I had to stand up for myself I would always think to myself how lucky people are that they're dealing with me and not you cause "deze meneer Kusmus hebben we al een keer meegemaakt". I love you so much Hisho. I'm so so proud to have the smartest little brother in the whole wide world. I can fly higher than an eagle, for you are the wind beneath my wings.

As cheesy as it is, I also want to thank myself. I have a tendency to always look ahead at the next challenge on my path without acknowledging just how far I've come and what I faced along the way. I've endured more during these last couple of years than anyone ever should during a lifetime. At some point I quit my PhD but eventually finished on antianxiety medication. I went from PhD counselor, to psychologist, to general practitioner, to bedrijfsarts and lastly to Human Resources. I navigated a difficult path between staying true to my values and self-worth while simultaneously attempting to achieve my goals. I was bent, severely, but luckily not broken. Never broken. And for that I thank myself. Because you know what Gigi? Remember that little girl who used to mix water with baby powder and vulpen ink trying to make potions? She's a real scientist now and she never lost her sense of wonder. 


\section{About the author}

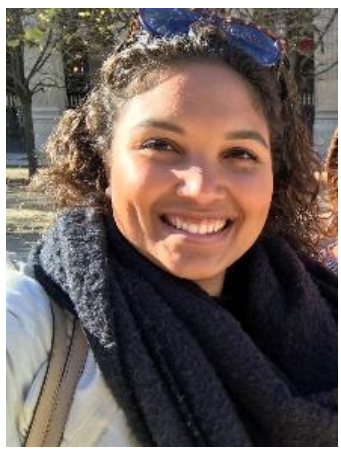

Gigi Kusmus was born Disraëli Kusmus on April $3^{\text {rd }} 1989$ on the Caribbean island of Curaçao. Here she did VWO at Radulphus college (high school) with a focus on science. At the age of 18 she moved to the Netherlands for her studies. In January 2009 she graduated from Hogeschool Utrecht with a HBO bachelors in chemistry, focused on research and development. For her first bachelor internship she spent six months in Ohio at Wright State University working in polymer chemistry. The second internship was done at Vrije Universiteit in Amsterdam performing multi-step synthesis in efforts to make an inhibitor for EPHA4 receptor tyrosine kinase. In June 2016 she received her master's degree in organic chemistry from Vrije Universiteit Amsterdam. During her master's studies she interned at Massey University in New Zeeland synthesizing [2.2]paracyclophane derivatives to serve as catalysts. During a short collaboration between UbiQ Bio and University of Amsterdam she synthesized a fluorogenic substrate based on Rhodamine110. In June 2017 she joined the group of Jos Paulusse at Twente University to pursue her $\mathrm{PhD}$ in polymer chemistry. Here she worked on polymer nanogels and their surface modification to serve as versatile platforms in biomedical applications. 